\title{
Synchronie und Diachronie
}

\section{Herausgegeben von}

Barbara Sonnenhauser

Caroline Trautmann

Daniel Holl

Patrizia Noel Aziz Hanna

\section{Redaktion}

Barbara Sonnenhauser

Marisa Eberle 


\section{Vorwort}

Mit der erstmaligen Ausrichtung in Zürich hat das „Diskussionsforum Linguistik in Bayern“ seinen Namen in „Diskussionsforum Linguistik“ geändert. Nicht geändert jedoch hat sich die Intention, Doktoranden und Habilitanden sämtlicher linguistischer Richtungen Gelegenheit zur Präsentation eigener aktueller Arbeiten zu geben.

Die im Tandem-Verfahren begutachtete Reihe Diskussionsforum Linguistik / Bavarian Working Papers in Linguistics ist weiterhin das Resultat der Tagungen. Im vorliegenden sechsten Band werden unter dem Rahmenthema „Synchronie und Diachronie“ sieben Aufsätze mit unterschiedlichen sprachlichen und methodischen Schwerpunkten versammelt.

Für die finanzielle Unterstützung zur Durchführung der Tagung im Mai 2016 danken wir dem Doktoratsprogramm Linguistik der Universität Zürich sowie der Graduiertenschule Sprache \& Literatur / Klasse für Sprache der LMU München; für die Hilfe bei der Vorbereitung des vorliegenden Bandes danken wir Marisa Eberle. Bedanken möchten wir uns auch bei den Beitragenden zu diesem Band sowie bei allen Vortragenden und Teilnehmern am Diskussionsforum.

Zürich, 12. Februar 2017

Barbara Sonnenhauser

Caroline Trautmann

Daniel Holl

Patrizia Noel Aziz Hanna

„Synchronie und Diachronie“

Diskussionsforum Linguistik / Bavarian Working Papers in Linguistics 6 (2017)

Universität Zürich

Ludwig-Maximilians-Universität München

Otto-Friedrich-Universität Bamberg

Februar 2017

ISSN: 2194-2439

Download: https://epub.ub.uni-muenchen.de/view/subjects/13282.html 


\section{Inhaltsverzeichnis}

Das Supinum in der slovenischen Grammatikschreibung - Gebrauch folgt Beschreibung oder Beschreibung folgt Gebrauch?

Martin Junge (Zürich)

Continuatio, imitatio, renovatio? Die Verb-Subjekt-Linearisierung in Machiavellis

Istorie fiorentine

Laura Kersten (Erlangen)

Zu germ. ${ }^{*} \overline{\mathrm{e}}^{1}$ im Präteritum der germanischen starken Verben

Patrick Mächler (Zürich)

Kommunikationsstrategien ungeübter Schreiber - Eine exemplarische Studie anhand eines französischen Bittbriefs

Stephanie Massicot (Erlangen)

Die Verwendung der perfektiven Präsensformen im Russischen, Slovenischen und modernen Resianischen

Malinka Pila (Konstanz)

Das sogenannte jako recitativum und seine paradigmatischen Verhältnisse zu übrigen Mitteln der altkirchenslavischen Syntax

Pino Marco Pizzo (Mainz)

Das Verständnis von Vulgärlatein in der Renaissance im Rahmen der Diskussion um das vulgare der römischen Antike vor dem Hintergrund der questione della lingua Roger Schöntag (Erlangen) 



\title{
Das Supinum in der slovenischen Grammatikschreibung - Gebrauch folgt Beschreibung oder Beschreibung folgt Gebrauch?*
}

\author{
Martin Junge (Zürich)
}

\begin{abstract}
Der vorliegende Aufsatz hat vor allem das Supinum im Slovenischen zum Gegenstand. Zum einen wird die Supinalkonstruktion als solche vorgestellt, zum anderen wird die Entwicklung in den Grammatiken und Lehrbüchern aufgezeigt. Dies erfolgt aus zweierlei Perspektiven: Anhand der Analyse der Grammatikschreibung des Slovenischen, zudem werden beschriebene Fakten aus den Grammatiken anhand der zur Verfügung stehenden Korpora überprüft und so ein Abgleich zwischen der Grammatikschreibung und dem Sprachgebrauch ermöglicht. Von besonderem Interesse sind dabei zum einen die Definition des Bewegungsverbes und zum anderen die Rektion mit den Kasus Genitiv und Akkusativ als auch die Frage des Verbaspektes im Supinum.
\end{abstract}

\section{Einleitung}

Als eine von nur wenigen slavischen Sprachen hat das Slovenische das Supinum, eine infinite Verbform, bis heute bewahrt. Es ist ausserdem noch im Niedersorbischen und im Kajkavischen zu finden. Auch in anderen Slavinen war es zu finden, teilweise tritt es als Relikt noch in festen Wendungen auf, beispielsweise im Tschechischen. ${ }^{1}$

Es handelt sich beim Supinum um eine infinite Verbform, die nach Verben steht, die eine Bewegung beschreiben. Der Begriff Verben der Bewegung griffe allerdings zu kurz, wie noch aufzuzeigen ist. Mithilfe des Supinums wird der Zweck einer Bewegung näher bestimmt und erläutert, die deutschen Begriffe hierfür lauten 'Zweckform' (wegen des Bezuges auf den Zweck einer Bewegung) oder 'Lagewort' (weil sich das Supinum stets an ein anderes Wort 'lehnt', das heisst nur in Verbindung mit einem Verb der Fortbewegung auftreten kann). Das Supinum wird vom Infinitiv eines Verbes gebildet, indem die Infinitivendung - $t i$ um das auslautende $-i$ gekürzt wird (vgl. dela $+\mathbf{t i} .^{\text {INF }}>$ dela $+\mathbf{t} .^{\text {SUP }}$ ).

Der Verlauf der Entwicklung der Beschreibung des Supinums im Slovenischen ruft jedoch unweigerlich die Frage hervor, ob die Veränderungen bei den Beschreibungen des Supinums in den Grammatiken und Lehrbüchern einem Muster folgen? Dem sind die Daten aus den Korpora gegenüberzustellen, die Aufschluss über den tatsächlichen Sprachgebrauch geben. Anhand dieser Gegenüberstellung möchte ich darstellen, ob der Sprachgebrauch den

\footnotetext{
Dieser Beitrag ist im Rahmen des von dem Schweizerischen Nationalfonds SNF geförderten Projekts „Sprachbeschreibung als Filter und Prisma: Die 'Individualität' des Slovenischen“" an der Universität Zürich entstanden (http://www.slav.uzh.ch/de/forschung/sprachwissprojekte/snf_slovenisch.html; Projektnummer 10001B_162970/1).

1 Jdu spat (Supinum) vs. jdu spát (Infinitiv) (ich gehe schlafen, Übersetzung MJ) - es erfolgt eine Reduzierung der Vokalquantität.
} 
Grammatikschreibungen folgt oder die Grammatikschreibungen dem Sprachgebrauch. Welche Rolle bei der Beschreibung und Anwendung des Supinums spielen beispielsweise die Herkunft des jeweiligen Grammatikschreibers und die Vorkenntnisse anderer Sprachen und Grammatiken? Beide Fragestellungen betreffen zuvörderst die Fragen der Beschreibung des Kasus von Objekten des Supinums und der Bildung des Supinums auch von perfektiven Verben.

Anhand der zur Verfügung stehenden Grammatiken und Lehrbücher des Slovenischen soll nachfolgend aufgezeigt werden, wie sich die Beschreibung des Supinums und dessen Verwendungsregeln entwickelten und welche Veränderungen in der Beschreibung es im Laufe des Betrachtungszeitraumes der Grammatiken von 1584 bis 2006 gab. Die Auswertung von Daten zum Gebrauch des Supinums im Hinblick wird einer weiteren Publikation vorbehalten bleiben, wo nötig gebe ich auch im vorliegenden Aufsatz schon Korpusbelege an.

Anhand der zur Verfügung stehenden Grammatiken und Lehrbücher soll zudem geklärt werden, ob es sich um eine konsistente Entwicklung handelte und wie sich die Bildungsvarianten des Supinums veränderten und welchen Einflüssen das Supinum während den Jahrhunderten unterlag. Zudem wird $\mathrm{zu}$ untersuchen sein, inwiefern sich die Grammatikdarstellungen untereinander ähneln, widersprechen, bzw. welche Bezüge sich ergeben und inwiefern auch Grammatikdarstellungen anderer Sprachen eine Rolle gespielt haben könnten.

Für diese Abhandlung betrachte ich ausgewählte, frei zugängliche Grammatiken aus den Jahren 1584 bis 2006, sofern sie Aussagen zum Supinum enthalten. In wenigen Fällen haben die Grammatikschreiber oder deren Schüler bzw. Mitarbeiter die ursprünglichen Fassungen verbessert und erneut veröffentlicht, sodass ich dann auch die Beziehungen der unterschiedlichen Auflagen untereinander betrachten werde. Die Gliederung folgt den Jahrhunderten, wobei für das 19. Jh. bedingt durch die Vielzahl veröffentlichter Lehrbücher und Grammatiken eine weitere Unterteilung sinnvoll erschien.

\section{Bildung des Supinums}

Eine Supinalkonstruktion besteht aus mindestens zwei Verben, dem Verb der Bewegung und einem zweiten Verb, das Zweck bzw. Absicht der Bewegung näher spezifiziert. Bei diesem zweiten Verb ist vor allem der Verbaspekt von Interesse. Diese Konstruktionen sind zudem im Hinblick auf den durch sie regierten Kasus (Akkusativ und Genitiv) relevant. ${ }^{2}$

Transitives Verb und Akkusativobjekt:

$$
\begin{aligned}
& \text { Pišem besedilo. } \\
& \text { schreibe. VIIS Text. } \\
& \text { 'Ich schreibe einen Text.' } \\
& \text { (eigenes Beispiel) }
\end{aligned}
$$

Verb der Bewegung und Verb im Supinum:

\footnotetext{
Auch im Tschechischen hat sich die Verwendung des Supinums mit Genitivobjekt prijde soudit živých i mrtvých anstelle prijde soudit živé i mrtvé 'er kommt um die Lebenden und Toten zu richten' (Koupil 1947: 79) (Übersetzung MJ) in einem Phrasem erhalten.
} 
$(2)$

$$
\begin{aligned}
& \text { Grem pisat. } \\
& \text { gehe. }{ }^{\text {VB1S }} \quad \text { schreiben. } \\
& \text { 'Ich gehe schreiben.' } \\
& \text { (eigenes Beispiel) }
\end{aligned}
$$

Verbindung von Verb der Bewegung, Supinum und Genitivobjekt:

$$
\begin{aligned}
& \text { Grem pisat besedila. } \\
& \text { gehe. }^{\text {VB1S }} \text { schreiben. }{ }^{\text {SUP }} \text { Text. }{ }^{\text {GEN }} \\
& \text { 'Ich gehe einen Text schreiben.' } \\
& \text { (eigenes Beispiel) }
\end{aligned}
$$

Verbindung von Verb der Bewegung, Supinum und Akkusativobjekt, wie es heute in der Standardsprache normiert ist (Toporišič 2004: 402)

$$
\begin{aligned}
& \text { Grem pisat } \quad \text { besedilo. } \\
& \text { gehe. }^{\text {VBIS }} \text { schreiben. }{ }^{\text {SUP }} \text { Text. } \\
& \text { 'Ich gehe einen Text schreiben.' } \\
& \text { (eigenes Beispiel) }
\end{aligned}
$$

Das Verb, von dem das Supinum gebildet wird, ist dabei zumeist imperfektiv, auf den Verbalaspekt wird später noch einzugehen sein.

\section{Das Supinum in den Grammatikschreibungen}

\subsection{6.-18. Jahrhundert}

In den ersten Grammatiken des Slovenischen, bzw. dessen einzelnen Dialekten, wird das Supinum nur kurz charakterisiert in Bezug auf Gebrauch und Bildung; so wird es in der Grammatik von Bohorič aus dem Jahre 1584 als in der Funktion dem Lateinischen ähnlich beschrieben und zur Bildung angemerkt, dass man das Supinum erhalte indem man das $-i$ der Infinitivendung weglässt (Bohorič 1584: 100). Bohorič nennt keine Beispiele zum Kasusgebrauch. Die Grammatik Bohoričs ist laut Kopitar (1809: XL) nichts weiter als eine Interpretation der lateinischen Grammatik Philipp Melanchthons, dessen Schüler er war. Dies könnte den fehlenden Bezug zum Slovenischen und insbesondere das Fehlen von Beispielen im Slovenischen durchaus erklären, zumal Bohorič nicht beschreibt, inwiefern sich das Supinum aus dem Lateinischen dem Slovenischen ähnelt, bzw. wo Unterschiede zu finden sind.

Etwas ausführlicher ist die Grammatik ,Slovennska Grammatika“ von Zagajšek, die mehr als 200 Jahre nach der Grammatik Bohoričs erschien. Er beschreibt das 'Supinum oder Lagewort' wie folgt:

Das Lagewort oder Supin wird mit Auslassung des letzten i in der unbestimmten Form gemacht, als: seyn, bit. (Zagajšek 1791: 126)

Die unbestimmte Form entspricht dabei der infiniten Verbform, 'unbestimmt' meint hier im Hinblick auf Genus, Numerus und Person sowie Genus verbi. 


\subsection{Jahrhundert}

\subsubsection{0-1850}

Jernej Kopitar sieht sich mit seiner „Grammatik der Slavischen Sprache in Krain, Kärnten und Obersteiermark" in der Tradition von Josef (Abbé) Dobrovský, der, so Kopitar weiter, das gesamte slavische Sprachwesen ,in kritische Revision“ nehmen wolle (Kopitar 1809: XXVIII) und betrachtet seine eigene slovenische Grammatik als „kleinen Beytrag zu diesem großen Unternehmen“ (1809: XXIX). Die kritische Revision Dobrovskýs, welche Kopitar anführt, ist im Kontext zur Überarbeitung der tschechischen Schriftsprache zu sehen, die Dobrovský vornahm. Er forschte und schrieb zur Geschichte der tschechischen Literatur und Sprache, dabei verfasste er das 1792 veröffentlichte Werk „Geschichte der böhmischen Sprache und Literatur“ und 1809 schließlich das „Ausführliche Lehrgebäude der tschechischen Sprache“, einer Grammatik des Tschechischen. Dobrovský gilt als Begründer der modernen tschechischen Schriftsprache, wählte aber bewusst das Tschechische des 16. Jh. als Grundlage für sein Schaffen in der Grammatikschreibung. Er widmete sich darüber hinaus auch anderen slavischen Sprachen und gab Zeitschriften heraus, die sich allgemein mit den slavischen Sprachen und deren Aufbau befassen. Aus diesem umfangreichen Wirken Dobrovskýs heraus, versucht Kopitar dessen Tradition zu folgen und so die slovenische Schriftsprache neu zu fassen.

Kopitar bezieht sich weiter unter anderem auf Bohorič und dessen Grammatik als ältester Sprachbeschreibung des Slovenischen (1809: XL) und stellt fest, dass sich die Sprache von Krain, Kärnten und der Steiermark seit der Veröffentlichung von dessen Grammatik gar nicht geändert habe (ebd.).

Des Weiteren erwähnt Kopitar mit Anton T. Linhart einen bedeutenden slovenischen Schriftsteller und Vertreter der Aufklärung sowie dessen Werk „Versuch einer Geschichte von Krain und der übrigen Länder der südlichen Slaven Österreichs“" (1809: XLVI), der ersten umfassenden Darstellung der Geschichte des heutigen Sloveniens und natürlich dessen Sprache, und merkt Fehler Linharts an, der behauptete, dass die Slovenen noch im 16. Jh. in Glagoliza schrieben (1809: XXXVIII). Zudem verweist Kopitar auf eine Grammatik in Linharts Buch, nämlich die (unvollständig gebliebene) Grammatik Kumerdeys, der ausser dem Slovenischen auch andere slavischen Sprachen in einer Grammatik verglich (ebd.), die jedoch nicht veröffentlicht wurde. ${ }^{3}$

Gerichtet ist die Grammatik Kopitars nach eigener Aussage vor allem an Volkslehrer und slavische Sprachforscher (1809: XLVII), er erwähnt dies jedoch nur, weil er gewisse Definitionen auslassen konnte und eben nur eine Grammatik, jedoch keine Sprachlehre verfasste (1809: XLVIII).

Das Supinum beschreibt Jernej Kopitar als „ein verkürzter Infinitivus, der der Bedeutung nach dem Lateinischen Supino entspricht" (1809: 331). Er weist darauf hin, dass das Supin im Slovenischen dieselben Funktionen erfüllt wie das lateinische Supin und dass es auch genauso verwandt wird. Phonetisch hingegen gebe es kaum einen Unterschied zwischen Infinitiv und Supinum:

Der Grieche weiß nichts vom Supino, das Lateinische wird für ein Substantivum gehalten und das unsrige wird wohl nichts anders seyn, als der Infinitivus ohne das End-i, so ohnehin, auch wenn es da ist, stumm lautet. Aber wahr ist's, daß in den Fällen, wo die Lateiner ihr Supinum,

3 Kumerdey, B.: Versuch über krainerische Rechtsschreibung (1779) und Krainisch-slavische Grammatik (1793) 
wir diesen Infinitiv ohne End-i gebrauchen. Das Französische Supin ist freylich ganz was andres, so wie ihr gérondif nicht das gerundium der Lateinischen Grammatik ist. Bald wird die grammatische Terminologie so voll von Synonymen seyn, wie die Naturhistorische. (Kopitar 1809: 331)

Auch Kopitar grenzt die Verben nicht ein, von denen das Supinum gebildet werden kann, noch nennt er einen Kasus, der durch das Supinum regiert wird.

Nur indirekt charakterisiert Professor Valentin Vodnik in seiner „Pifmenoft ali Gramatika sa Perve Shole“ (1811) das Supinum: Für eine bessere Aussprache lasse man unter anderem bei Verben, die Verben der Bewegung folgen, das $i$ an der Endung des Infinitives weg (Vodnik 1811: 128). Vodnik beschreibt damit als Erster explizit, dass das Supinum den Verben der Bewegung folgt, sieht man davon ab, dass Kopitar die Funktion des Supinums als dem Lateinischen gleich definierte.

Mit dem Erscheinen der Grammatiken und Lehrbücher ab 1820 werden die Ausführungen im Allgemeinen und zum Supinum im Besonderen ausführlicher und detaillierter.

Peter Dajnko, ein Priester aus der Untersteiermark, definiert das Supinum in seinem „Lehrbuch der Windischen Sprache“ zunächst als Lagewort und Zwecksform (Dajnko 1824: 281), bevor er erläutert, dass in Supinalsätzen „das, was man vornimmt in den Genitiv [kommt]" (ebd.). Dajnko zeigt dies anhand eines Beispiels, in dem delavcov 'Arbeiter' als Objekt zu zvat 'rufen' im Genitiv steht:

$$
\begin{array}{lll}
\text { Idi } & \text { delavcov } & \text { zvat. } \\
\text { geh. VBIMPER } & \text { Arbeiter. }{ }^{\text {GENPL }} & \begin{array}{l}
\text { rufen. } \\
\text { 'GuP }
\end{array} \\
\text { 'Geh die Arbeiter rufen!' } & \\
\text { (Dajnko 1824: 281) } &
\end{array}
$$

Dajnko ergänzt zudem, dass der Gebrauch des Supinums mit dem Genitiv nicht ohne Ausnahmen sei, sodass in sehr vielen Supinalsätzen der Akkusativ stehe, insbesondere nach den im Beispiel in der ersten Person Singular angegebenen Verben wie dam/davam 'ich gebe', denem/devam 'ich tue etwas wohin', nesem/nosim 'ich trage', pelam/vozim 'ich führe', poslem/posilam 'ich schicke', verxem/mečem 'ich werfe' (1824: 281), die keine Bewegungsverben sind. Dajnko führt dabei aber nicht weiter aus, was diese Ausnahmen begründet, wodurch sich unweigerlich die Frage stellt, warum gerade die Verben, die keine Verben der Bewegung sind, nicht den Genitiv regieren, sondern den Akkusativ.

Interessanterweise sind für die von Dajnko benannten Ausnahmen im IMP-Korpus (http://nl.ijs.si/noske/sl-ref.cgi/first_form), das ältere Texte des Slovenischen vom 15. Jh. bis zu Beginn des 20. Jh. enthält, überhaupt kaum Supinalsätze zu finden, sehr deutlich überwiegend steht der Infinitiv nach diesen Verben, lediglich für voziti 'führen' und poslati/pošiljati 'schicken' lassen sich einige wenige Beispiele mit dem Supinum finden.

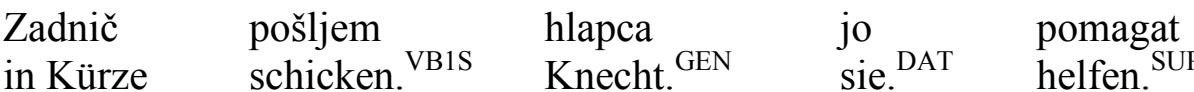

$$
\begin{aligned}
& \text { 'In Kürze schicke ich einen Knecht um ihr zu helfen.' } \\
& \text { (1847, Čujte, čujte, kaj žganje, IMP) }
\end{aligned}
$$

Durch die geringe Anzahl an Belegen - es handelt sich um weniger als zehn für alle von Dajnko als Ausnahme genannten Verben der Bewegung insgesamt - lässt sich zum Kasusgebrauch keine allgemeine Aussage machen; mit dem vorwiegenden Gebrauch des Infinitivs 
ist in der Konsequenz der Akkusativ als Kasus vorherrschend, da diese Verben in Verbindung mit dem Infinitiv den Akkusativ regieren.

Dajnko unterscheidet auch zwischen unbestimmten und bestimmten Zeitwörtern, wobei er das unbestimmte Verb charakterisiert als „wenn ohne Person und Zahl gesprochen wird“ (Dajnko 1824: 196); es entspricht also einer infiniten Verbform. Dajnko ist der Erste, der die Kasusrektion des Supinums definiert und dies sowohl für den hauptsächlichen Gebrauch mit dem Genitiv als auch mit den Ausnahmen, die den Akkusativ regieren können. In Ergänzung erläutert Dajnko die Bildung des Lagewortes:

Dieses Nebenstück unbestimmter Art [gemeint ist das Supinum, MJ] ist nur nach solchen Zeitwörtern anwendbar, die eine Bewegung von einem Orte zum andern anzeigen, und selbst nach diesen nur dann, wenn das unbestimmte Zeitwort kein Perfectives ist. (Dajnko 1824: 196)

und weiter im Kapitel über die Wortfügung: „Zeigt das bestimmte Zeitwort eine Bewegung von einem Orte zum andern, so wird das unbestimmte, wenn es ein Imperfectives ist, in das Lagewort gesetzt [...] (1824: 284)

Im Unterschied zu Vodnik schreibt Dajnko also nicht von den Verben der Fortbewegung als einer bestimmten Gruppe von Verben, sondern von jenen Verben, die eine reale Ortsveränderung bezeichnen. Unter diesem Aspekt ist auch die Rektion der o.g. Verben mit dem Akkusativ zu sehen, jedenfalls kennzeichnen die angeführten Verben zumeist keine Ortsveränderung, anders als beispielsweise iti 'gehen', hoditi 'gehen' oder teči 'laufen'.

Zudem steht das Supinum Dajnko gemäß nicht nach Verben, die eine Bewegung anzeigen, allerdings nicht in diesem Sinne gebraucht werden. Anstatt dem Supinum stehe dann der Infinitiv (1824: 285). Im Beispiel (7) beschreibt hiteti 'eilen' nur das Verb delati 'tun, arbeiten' näher, erfüllt also die Funktion eines Adverbs und zeigt keine eigentliche Bewegung an.

$$
\begin{array}{lll}
\text { Ja hitim } & \text { delati. } \\
\text { Ich } & \text { eile. }{ }^{\text {VIS }} & \text { machen. }
\end{array}
$$

'Ich beeile mich zu arbeiten. / Ich arbeite schnell.'

(Dajnko 1824: 285)

Wird mittels des Verbes der Bewegung eine Tätigkeit näher beschrieben oder in ihrer Ausführung charakterisiert oder wird durch das Verb der Bewegung keine Ortsveränderung bezeichnet steht der Infinitiv anstelle des Supinums.

Dass es auf den Kontext bzw. auf eine tatsächlich stattfindende Bewegung mit Ortsveränderung ankommen könnte, lässt sich am folgenden Beispiel darstellen:

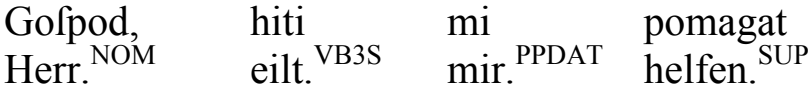

$$
\begin{aligned}
& \text { 'Herr, eile mir zur Hilfe.' } \\
& \text { (1844, Zlata Jabolka, IMP) }
\end{aligned}
$$

In diesem Fall steht nach hiti das Supinum, was dadurch erklärbar ist, dass hier eine Bewegung im Vordergrund steht: Jemand muss eine Ortsveränderung vornehmen, damit er helfen kann. Hiteti beschreibt hier keine Tätigkeit, pomagati 'helfen' näher, sondern steht für sich als Bewegung. Die gleiche Textstelle findet sich allerdings auch ohne Supinum bereits ca. 70 Jahre zuvor: 


$$
\begin{aligned}
& \begin{array}{l}
\text { Gospud hiti } \\
\text { Herr. }
\end{array} \text { eilt. }{ }^{\text {VB3S }} \begin{array}{l}
\text { meni } \\
\text { mich. }
\end{array}{ }^{\text {PAKK }} \begin{array}{l}
\text { pomagati. } \\
\text { helfen. }
\end{array} \\
& \text { 'Herr, eile mir zur Hilfe.' } \\
& \text { (1777, Branja in evangeliji, IMP) }
\end{aligned}
$$

Die Konstruktion von hiteti und Supinum bedarf noch einer genaueren Untersuchung, insbesondere die Anwendung je nach Bedeutung von hiteti als Verb der Bewegung oder als beschreibendes Verb müsste ausgewertet werden. Es zeigt sich aber anhand der Korpusdaten, dass der Gebrauch sich während der Jahre verändert.

Für die Verbindung von Supinum und Akkusativ nennt Dajnko ebenso Beispiele, wie (10):

$$
\begin{aligned}
& \text { Dekla dene vodo segravat. } \\
& \text { Magd. }{ }^{\text {NOM }} \text { tun. }^{\text {V3S }} \text { Wasser. }^{\text {AKK }} \text { erwärmen. }{ }^{\text {SUP }} \\
& \text { 'Die Magd setzt Wasser auf.' } \\
& \text { (Dajnko 1824: 281) }
\end{aligned}
$$

Das Verb deti 'tun' ist ebenso wie die bereits angeführten Ausnahmen kein Verb der Bewegung. Im IMP-Korpus finden sich nur zwei Evidenzen für ein Supinum nach deti.

Für unbestimmte, perfektive Verben beschreibt Dajnko das Folgende:

Ist aber das unbestimmte Zeitwort ein Perfectives, so steht es nie im Lageworte, sondern muß mit seinem bestimmten Bewegungszeitworte in gleiche Person, Zahl, Zeit, Art und Bedeutung zu stehen kommen [...]. (Dajnko 1824: 285)

Das ist für die Zeit Dajnkos wohl noch zutreffend, andererseits lassen sich ab Mitte des 19. Jh. viele Belege für die Verbindung von Bewegungsverb und Supinum eines perfektiven Verbs finden, beispielsweise:

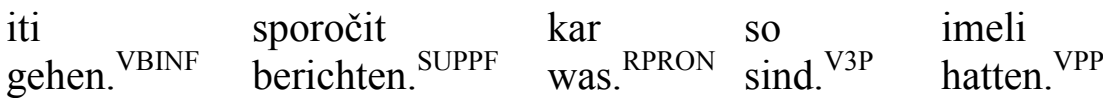

$$
\begin{aligned}
& \text { 'Gehen um zu berichten, was sie hatten.' } \\
& \text { (1853, Divij huni pred mestom, IMP) }
\end{aligned}
$$

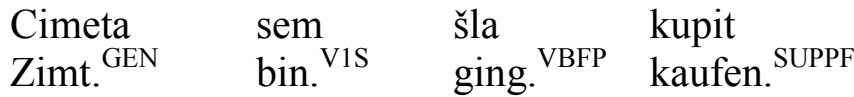

$$
\begin{aligned}
& \text { 'Zimt ging ich kaufen.' } \\
& \text { (1895, Jarem pregrehe, IMP) }
\end{aligned}
$$

Dajnko beschreibt zusammenfassend das Supinum mit Genitivobjekt als nach Verben stehend, die eine tatsächliche Bewegung von A nach B beschreiben. Es kann nur von imperfektiven Verben gebildet werden. Nach einigen Verben, die keine Verben der Bewegung sind, steht davon abweichend ein Akkusativobjekt.

Nach dem Vorbild Josef Dobrovskýs „Lehrgebäude der böhmischen Sprache“ aus dem Jahre 1809 erscheint im Jahr 1825 eine Grammatik des Geistlichen Franc Metelko, welche er „Lehrgebäude der slovenischen Sprache im Königreiche Illyrien und in den benachbarten Provinzen nach dem Lehrgebäude der böhm. Sprache des Hrn. Abbé Dobrowsky“ nennt. Außer auf Dobrovský (Metelko 1825: XXVIII) bezieht er sich in seinem Lehrbuch auch auf Kopitar und dessen Grammatik (1825: XXVII). 
Metelko (1825: 257) schreibt zum Supinum: „Auf die Zeitwörter einer Bewegung folgt nicht der Infinitiv, sondern das Supinum.“

In der Frage der Rektion des Supins mit den Kasus beschreibt er die Verbindung von Supinum und Genitiv wie folgt: „In andern Dialekten regiert auch das Supinum den Genitiv“ (1825: 244) jedoch als in der Krain nicht vorkommend, dessen ,ungeachtet es in der Steyermark üblich zu seyn scheint" (ebd.). Ohne das Beispiel näher zu erläutern, führt Metelko in einer Übersicht das Supinum sowohl von einem imperfektiven dvigati 'heben' als auch von einem perfektiven Verb dvigniti 'heben' an (1825: 132).

Metelko stellt also einen Zusammenhang zwischen Kasusverwendung (er selbst stammt aus der Krain und verwendet den Akkusativ) und Dialekten her und gibt Hinweise auf den Gebrauch des Supinums mit perfektiven und imperfektiven Verben.

Franc Metelko zitiert zudem den Juristen Janez Šmigoc (auch Schmigoz) aus der Untersteiermark und dessen Lehrbuch und Grammatik „Windische Sprachlehre“ aus dem Jahre 1812, in dem es durchaus Evidenzen für den Genitiv regierende Supinalsätze gebe (ebd.).

Tatsächlich beschreibt Šmigoc (1812: 132) in seiner „Theoretisch-praktischen Sprachlehre“ ausschließlich die Konstruktion aus Supinum und Genitivobjekt: „Alle Zeitwörter, wenn sie im Lageworte stehen, das ist, in der abgekürzten unbestimmten Art fordern, daß ihr Hauptwort in den Genitiv gesetzt wird; [...]“. Das Lagewort als solches erläutert Šmigoc derart, dass unbestimmte Zeitwörter die nach Verben stehen, welche eine Bewegung von einem Ort zum anderen aussagen, in das Lagewort gesetzt werden müssen, unter anderem nach den Verben gehen, kommen, schicken, sich begeben usw. (1812: 133). Er schließt sich damit Dajnkos Auffassung an, dass das Verb der Bewegung allein als Kriterium für die Verwendung des Supinums nicht genügt, es muss tatsächlich eine Bewegung mit Ortsveränderung beschrieben werden.

Meines Erachtens entspricht diese Auffassung auch am ehesten der Funktion des Supinums, nämlich den Zweck eine Bewegung näher zu beschreiben und eben nicht eine andere Tätigkeit mithilfe dieser Verben näher zu charakterisieren oder die Tätigkeitsausübung zu beschreiben.

Šmigoc (1812: 133) führt lediglich ein Beispiel an, welches die Rektion mit dem Genitiv zeigt:

$$
\begin{aligned}
& \text { Prisem rib lovit. } \\
& \text { Komme. }{ }^{\text {VBIS }} \text { Fische. }{ }^{\text {GEN }} \text { jagen. }{ }^{\text {SUP }} \\
& \text { 'Ich komme um Fische zu jagen.' (Übersetzung Šmigoc) } \\
& \text { (Šmigoc 1812: 133) }
\end{aligned}
$$

Sieben Jahre nach Metelko, im Jahre 1832, veröffentlicht der Sprachwissenschaftler Anton Johann Murko seine Grammatik „Theoretisch-Praktische Slovenische Sprachlehre“. Gebürtig stammt er wie Peter Dajnko aus der Untersteiermark. Er fasst die Bildung des Supinums und die Rektion desselben mit Nomen deutlich konkreter als Dajnko und widerspricht ihm insoweit, als dass er die Verbindung von Supinum und Akkusativ nicht für zulässig hält, „Alle Zeitwörter, wenn sie im Supin stehen, haben den Genitiv nach sich.“(Murko 1832: 124)

Er schließt sich also Šmigoc' Auffassung an und revidiert Dajnkos Ansichten (ebd.).

$$
\begin{aligned}
& \begin{array}{lll}
\text { Grem } & \text { rib } & \text { lovit. } \\
\text { gehe. }^{\text {VBIS }} & \text { Fische. }^{\text {GEN }} & \text { jagen. }
\end{array} \\
& \text { 'Ich gehe um Fische zu jagen.' } \\
& \text { (Murko 1832: 124) }
\end{aligned}
$$


Murko beschreibt in seiner Grammatik das Supinum als eine Form des unbestimmten Verbes, die man erhält, wenn man von Verben das - $i$ der Infinitivendung weglässt. Es steht nach ,grém 'ich gehe', hódim 'ich pflege zu gehen', potújem 'ich reise', hitim 'ich eile', póshlem 'ich schicke"“ und „m. dgl. [mehr dergleichen, MJ] Bewegungszeitwörtern“ (Murko 1832: 76).

Als Beispiel führt Murko (1832: 76) unter anderem an:

$$
\begin{array}{lll}
\text { Ja hitim } & \text { delat. } \\
\text { ich } & \text { eile. }^{\text {VB1S }} \text { machen. }
\end{array}
$$

'Ich beeile mich zu arbeiten.'

Hier findet sich erneut ein Widerspruch zu Dajnkos „Lehrbuch der Windischen Sprache“, der genau an diesem Beispiel illustrierte, dass hier nicht das Supinum stehen könne, weil keine tatsächliche Bewegung vorliegt, sondern das Verb der Bewegung nur ein anderes Verb näher beschreibt (vgl. 1824: 285), vgl. (6) oben. Murko hingegen unterscheidet offenkundig nicht danach, ob das Verb der Bewegung auch eine tatsächliche Ortsveränderung beschreibt, sondern wendet das Supinum zumindest bei hiteti unabhängig vom Kontext an.

Anton Murko veröffentlichte in der Folge noch zwei weitere Ausgaben seiner „Theoretisch-Practischen Sprachlehre“ in den Jahren 1843 und 1850. Seine Ansichten zum ausschließlichen Gebrauch mit dem Genitiv ändert Murko nicht (vgl. 1843: 154, 1850: 125), allerdings beschreibt er explizit die Möglichkeit, von perfektiven und imperfektiven Verben das Supinum zu bilden, was in der Erstausgabe zwar nicht verneint wird, allerdings auch nicht erwähnt wurde (vgl. 1843: 80, 1850: 72). Die Frage der Bildung des Supinums von perfektiven Verben stellte sich 1832 also für Murko scheinbar noch gar nicht, während es 1843 und 1850 anscheinend Klärungsbedarf bezüglich der Bildung des Supinums auch von perfektiven Verben gab, wobei unklar bleibt, warum er diese Überarbeitung vornahm. Diese Entwicklung bedarf in der Zukunft einer weiteren Untersuchung, da sich größere Veränderungen abzeichnen, die sich anhand Murkos drei Ausgaben nachzeichnen lassen könnten.

Tatsächlich ist in den Korpora erkennbar, dass es von der Mitte des 19. Jh. an eine Entwicklung gab, das Supinum auch von perfektiven Verben zu bilden. Eine Abfrage im IMPKorpus mit teči 'laufen' ergab eine Evidenz bereits für das Jahr 1830:

$$
\begin{aligned}
& \begin{array}{lllll}
\text { Rahela } & \text { bersh } & \text { tezhe } & \text { povedat } & \text { ozhetu. } \\
\text { Rahela. }^{\text {NAME }} & \text { bersh. }^{\text {ADV }} & \begin{array}{l}
\text { tezhe. } \\
\text { VB }
\end{array} & \begin{array}{l}
\text { povedat. } \\
\text { SUPF }
\end{array} & \begin{array}{l}
\text { ozhetu. } \\
\text { DAT }
\end{array}
\end{array} \\
& \text { 'Rahel läuft schnell, um es dem Vater zu erzählen.' } \\
& \text { (1830, Zgodbe Svetega pisma za mlade ljudi, IMP) }
\end{aligned}
$$

Es muss also während den Jahren neue Erkenntnisse für Murko gegeben haben, um die Kriterien weiter $\mathrm{zu}$ fassen und auf die mögliche Verwendung von perfektiven Verben explizit hinzuweisen.

\subsubsection{1-1900}

Der Kärntner Philologe Anton Janežič veröffentlichte 1863 seine „Slovenska slovnica domače in šolsko rabo“ und beschreibt das Supinum (namenivnik, namenivni naklon, Janežič 1863: 236) unter den $\S \S 469$ und 470 folgendermaßen: Das Supinum wird verwendet nach Verben, die eine Bewegung von einem Ort zum anderen Ort beschreiben (ebd.). Janežič widerspricht so auch Murko insofern, als dass er durch die Spezifizierung des Bewegungsbegriffes (Bewe- 
gung von Ort zu Ort) meines Erachtens nach nicht die Verwendung des Supins bei hitim delat erlaubt, da hiteti 'eilen' in diesem Fall eben keine Bewegung, sondern die Art und Weise der Arbeit näher bezeichnet, ohne eine Ortsveränderung auszudrücken.

Außerdem steht das Supinum dann nach dati, wenn damit eine Bewegung gekennzeichnet wird, im Sinne von 'schicken, senden' (1863: 236). Die besondere Bedeutung von dati im Kontext mit dem Supinum ist daran ersichtlich, dass fast jeder Verfasser dieses Verb extra und außer der Reihe der Verben der Bewegung nennt und mit dem Supinum verbindet. Möglicherweise ist bei dati eine semantische Erweiterung sichtbar, die außer der eigentlichen Bedeutung 'geben' auch als Verbindung 'dati + Infinitiv' in Erscheinung tritt, was einen Einfluss auf den Gebrauch des Supinums in diesen Fällen haben könnte (vgl. Waldenfels 2012). ${ }^{4}$

Janežič vertritt allerdings ebenso wie Murko die Auffassung, dass das Supinum auch von vollendeten Verben gebildet werden könne, auch wenn man dies wo möglich vermeiden müsse (1863: 75). Das Supinum vollendeter Verben lasse sich zudem nur dann verwenden, wenn man eine einmalige Handlung ausdrücken möchte. Zum Kasusgebrauch schreibt Janežič, dass das Supinum oft den Genitiv regiert (1863: 184). Den Gebrauch des Supinums mit dem Akkusativ beschreibt er nicht, da er aber von einer häufigen (nicht ausschließlichen) Rektion des Supinums mit dem Genitiv schreibt, ist auch diese Variante im Umkehrschluss denkbar.

In einer von Jakob Sket aus der Untersteiermark redigierten Fassung der Grammatik von Janežič aus dem Jahr 1894 wird die Verwendung perfektiver Verben als „seltener“ beschrieben, die es dennoch zu meiden gilt (1894: 233). Sket beschreibt zudem den Gebrauch von Supinum und Genitiv, fügt aber an, dass öfters auch der Akkusativ verwendet werde (1894: 182).

Bereits drei Jahre nach Janežič, im Jahre 1866, erscheint in Laibach eine Grammatikbeschreibung vom wie Primus Trubar aus der Unterkrain stammenden Fran Levstik, die er „Die Slovenische Sprache nach ihren Redetheilen“ nennt. In dieser charakterisiert er das Supinum als eine um das auslautende i verkürzte 'Nennform', die nach den Verben der Bewegung stehe und meistens von 'Dauerzeitwörtern' (imperfektiven Verben, Anm. MJ) gebildet werde (Levstik 1866: 52).

Levstik gibt keine Erklärung, welchen Kasus das Supinum regiert, verwendet jedoch in seinem Beispiel den Akkusativ:

$$
\begin{aligned}
& \text { Kristus je prišel zakon doplnjavat. } \\
& \text { Christus. }^{\text {NOM }} \text { ist. }^{\text {VI3S }} \text { gekommen. }^{\text {VBP }} \text { Gesetz. }^{\text {AKK }} \text { erfüllen. }^{\text {SUP }}
\end{aligned}
$$

'Christus ist gekommen, das Gesetz zu erfüllen.'

(1866: 52, Übersetzung Levstik)

Zudem vertritt er die Ansicht Janežičs, dass in geringem Umfange das Supinum auch von perfektiven Verben gebildet werden könne, jedenfalls dann, wenn dies eine Tätigkeit mit nur kurzer Dauer bezeichnet, wie in (19) zu sehen ist (ebd.). Pobrati 'aufklauben ist perfektiv und bezeichnet eine nur kurz andauernde Tätigkeit.

$$
\begin{aligned}
& \text { Pojdi } \quad \text { oreh } \quad \text { pobrat. } \\
& \text { geh. }{ }^{\text {VBFUT }} \text { Nuss. }^{\text {AKK }} \text { aufklauben. }{ }^{\text {.UPPF }} \\
& \text { 'Geh die Nuss aufklauben!' } \\
& \text { (1866: 52, Übersetzung Levstik) }
\end{aligned}
$$

\footnotetext{
4 Die Untersuchung dieser möglichen Bedeutungserweiterung von dati bzw. der Abhängigkeit von dati und Supinum wird Gegenstand einer weiteren Arbeit sein.
} 
Šuman beschreibt das Supinum in seiner Schulgrammatik „Slovenska slovnica za srednje šole“, die sich an Beispiele von Miklošič hält, als eine besondere Form des Infinitives bei Verben der Bewegung mit der Endung auf $t t$ (Šuman 1884: 202). Bezüglich des Kasusgebrauchs beschreibt Šuman die Situation als von der Region abhängig: Im Osten, wo es üblich sei, das Supinum nur aus unvollendeten Verben zu bilden, stehe ausnahmslos der Genitiv; im Westen hingegen nicht (1884: 334). Hier gibt es überdies Aufschluss darüber, dass auch Šuman es als möglich erachtet, das Supinum von perfektiven Verben zu bilden, dies jedoch offenbar nur in gewissen (westlichen) Regionen und deren Dialekten.

\subsection{Jahrhundert}

Eine weitere Grammatik für Schulen stammt von Dr. Anton Breznik, der sein Werk von 1916 ebenso wie Šuman „Slovenska slovnica za srednje šole“ nennt. Das Supin beschreibt Breznik (1916: 153) als Verbform, mit der der Zweck einer Bewegung zum Ausdruck gebracht wird. Er bezeichnet das Supinum von imperfektiven Verben als 'echtes Supinum' und weist beim Kasusgebrauch darauf hin, dass die Verwendung mit dem Genitiv eine ältere Form sei, die sich jedoch in den östlichen Dialekten noch erhalten habe, ansonsten sei der Akkusativ zu verwenden (ebd.). Für das Verb dati 'geben' sei eine Verbindung mit dem Supin nur dann gegeben, wenn es im Sinne von 'etwas schicken', 'senden' verwandt wird (1916: 154).

Anders als Levstik stellt Breznik - der Position anderer Grammatikschreiber folgend fest, dass von perfektiven Verben kein Supinum gebildet werden könne, diese stehen nach einem Verb der Bewegung stets im Infinitiv und regieren den Akkusativ. Allerdings gesteht er doch zwei Ausnahmen zu, die Verben leči 'legen' und sesti 'setzen' (ebd.). Bei einer Korpusabfrage im IMP-Korpus für den Zeitraum 15. Jh. Bis zu Beginn des 20. Jahrhunderts ${ }^{5}$ ergab sich jedoch für sesti nicht ein einziges Supinum, für leči hingegen finden sich Supinalverbindungen, diese jedoch ausschließlich mit imperfektiven Verben. Insofern ist es schwierig nachzuvollziehen, was Breznik zur Erwähnung dieser Ausnahmen bewog. ${ }^{6}$ Explizit weist er darauf hin, dass sich vor allem Schriftsteller dieses Unterschieds nicht bewusst seien und im 'richtigen' Gebrauch - den er freilich nicht näher beschreibt - von Supinum und Infinitiv nicht kundig wären.

\footnotetext{
Abfrage für sesti:

http://nl.ijs.si/noske/all.cgi/view?q=aword\%2C\%5Blemma\%3D"sesti"\%5D\&corpname=imp\&attrs=word\&ct xattrs $=$ word $\&$ structs $=\&$ refs $=\% 3$ Dtext.title $\% 2 \mathrm{C} \% 3$ Dtext.date \&lemma $=$ sesti\&pagesize $=100 \&$ gdexcnt $=50 \& \mathrm{iq}$ uery $=$ vlak $\&$ fromp $=15 \&$ fromc $=$

Abfrage für leči:

http://nl.ijs.si/noske/all.cgi/first?corpname=imp\&reload=\&iquery=vlak\&queryselector=lemmarow\&lemma=l eči\&lpos $=\&$ phrase $=\&$ word $=\&$ wpos $=\&$ char $=\&$ cql $=\&$ default_attr $=$ word $\&$ fc_lemword_window_type $=$ right $\& f$

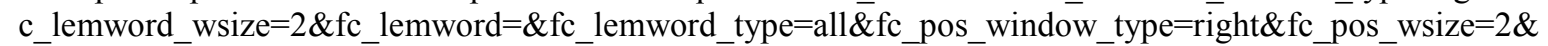
fc_pos $=$ glagol\&fc_pos_type $=$ all\&usesubcorp $=\&$ fsca_text.title $=\& f$ fsca_text.author $=\& f s c a \_t e x t . d a t e=\& f s c a \_p$. 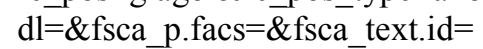 Abfragen am 21.12.2016

6 Im Korpus des heutigen Slovenischen (http://www.gigafida.net) gibt es für beide durch Breznik erwähnten Wörter zwar Evidenzen mit dem Supinum perfektiver Verben, jedoch nur in sehr geringer Anzahl.
} 
Breznik (1916: 154) zeigt die Verwendung des Supinums im Zusammenhang mit dem Imperativ auf und die Substitutionsmöglichkeit des Supinums durch einen Nebensatz mit der Partikel 'da' oder den Imperativ.

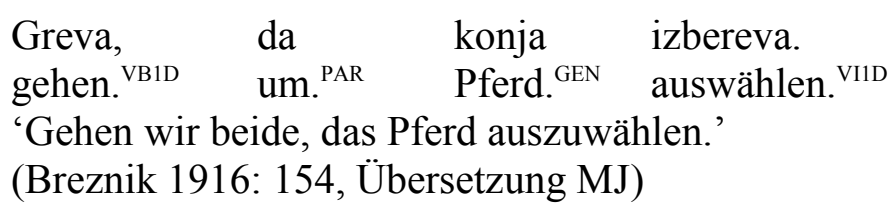

Dieser Zustand, wie von Breznik beschrieben, gilt im Wesentlichen bis heute, wobei sich insbesondere Svane (1958), Bajec et al. (1956) als auch Toporišič (1984) an Breznik orientieren.

Ausführlich beschreibt die „Slovenska slovnica“ aus dem Jahre 1956, die von Bajec, Kolarič, Rupel und Šolar (Bajec et al. 1956) verfasst wurde, das Supinum und dessen Verwendung, zum einen die Bildung, die einem Infinitiv ohne die Endung $-i$ entspricht, zum anderen aber erwähnen die Autoren auch, dass das Supinum in seltenen Fällen durchaus auch von perfektiven Verben gebildet werden könne (Bajec et al. 1956: 230), auch fassen die Autoren den Begriff des Verbes der Bewegung weiter: das Supinum stehe regelmäßig nach Verben der Bewegung oder Verben, die eine Bewegung indizieren (ebd.). Diese Erweiterung des Begriffes der Bewegung steht damit in der Tradition Murkos, muss sich doch lediglich eine Bewegung indizieren, das heißt erkennen lassen.

Das Supinum lässt sich nach Bajec et al. (1956) nicht konjugieren und ist unpersönlich, stellt also eine infinite Verbform dar. Ein Passiv werde vom Supinum nur in den Medien gebildet, ansonsten nicht (ebd.), die Autoren führen dazu allerdings kein Beispiel an. Das Autorenkollektiv weist auf den Gebrauch des Supinums nach dem Verb dati 'geben' hin (1956: 231). Aufschluss gibt die Grammatik aber auch über die Kasusrektion des Supins: Selbst wenn die Beispiele allesamt den Genitiv regieren, so gilt doch „Postavili bi ga lahko tudi v tožilnik, kar je danes redna raba v knjižnem jeziku“ 'Man könnte das Supinum auch den Akkusativ regieren lassen, so wie es heute in der Literatursprache üblich ist' (Übersetzung MJ) (1956: 231). Besonders bei älteren Schreibern und in den Dialekten der Steiermark sei der Gebrauch mit dem Genitiv aber noch sehr häufig (ebd.).

Nach Svane (1958: 138) regiert das Supinum in der modernen slovenischen Schriftsprache ein Akkusativobjekt, es finden sich nur dialektal und in älteren Sprachformen noch Konstruktionen mit einem Genitivobjekt. Das Supinum bildet man durch Entfernen des auslautenden $-i$ des Infinitives (1958: 77). Gebildet werden kann das Supinum nur von imperfektiven Verben, auch hier nicht von allen, wobei der Autor nicht näher ausführt, von welchen imperfektiven Verben das Supinum nicht gebildet werden. Schließlich wird mit Supinalkonstruktionen eine Absicht ausgedrückt (1958: 146). Außer nach den Verben der Bewegung wird auch nach dati 'geben' das Supinum verwandt, allerdings nur in der Bedeutung losschicken, schicken (1958: 147). Das Verb der Bewegung kann in bestimmten Fällen ausgelassen werden (1958: 146), das Supinum steht aber auch in den Ellipsen.

In den gegenwärtig aktuellsten Grammatiken zum Slovenischen, verfasst durch Jože Toporišič, wird das Supin als Verbform beschrieben, die regelmäßig nach den Verben der Bewegung auftritt (Toporišič 2004: 402). Das Verb der Bewegung kann jedoch elliptisch sein. Das Supinum kann auch dazu verwandt werden, eine gewisse Emotion in Fragen noch zu

Robert Grošelj indes beschreibt die Ersatzkonstruktion des Supinums in einigen slovenischen Dialekten nicht durch ' $d a+$ Infinitiv', sondern durch ' $z a+$ Infinitiv'. Die Variante ' $d a+$ Infinitiv' hingegen ordnet er štokavischen Dialekten zu (vgl. Grošelj 2014: 319). 
verstärken, was Toporišič nicht weiter ausführt, sondern als Beispiel nur „Kaj si jim pa šel pravit te stvari“" (ebd.) anführt.

Auch zur Beschreibung einer zweiten, auf der ersten fußenden Handlung wird das Supin verwendet; wie nach den Verben dati 'geben'und poslati 'schicken':

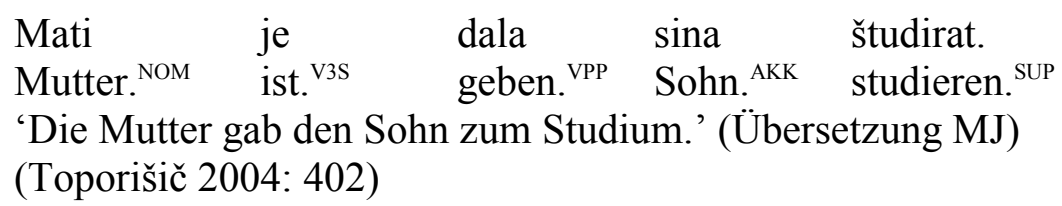

Toporišič beschreibt den Wandel bei der Bildung des Supinums; heute (das heißt zum Zeitpunkt des Erscheinens des Buches im Jahr 2004) bildet man es auch von vollendeten Verben. Er fügt außerdem hinzu, dass es „früher“ nicht möglich gewesen sei, das Supinum nur mit unvollendeten Verben zu bilden, weshalb es zum Ersatz des Supinums durch das Futur gekommen war (2004: 402).

$\begin{array}{llll}\text { Grem, } \quad \text { bom } & \text { pristavila } & \text { kosilo. } \\ \text { gehe. }^{\text {VB1S }} \quad \text { werde. }^{\mathrm{V} 1 \mathrm{~S}} & \begin{array}{c}\text { aufsetzen. } \\ \text { VPP }\end{array} & \begin{array}{l}\text { Essen. } \\ \text { 'IKK }\end{array} \\ \text { (Tophehe und werde das Essen aufsetzen.' } & \end{array}$

$\begin{array}{lll}\text { Grem } & \text { pristavit } & \text { kosilo. } \\ \text { gehen. }{ }^{\text {VBIS }} \quad \begin{array}{l}\text { aufsetzen. } \\ \text { SUP }\end{array} & \text { Essen. }{ }^{\text {AKK }} \\ \text { 'Ich gehe das Essen aufsetzen.' } & \\ \text { (Toporišič 2004: 402) } & \end{array}$

Das Objekt in Supinalsätzen steht nach Toporišič heute ganz überwiegend im Akkusativ anstelle des Genitivs (2004: 402).

Greenberg (2006) definiert die Verwendung des Supins als „,[f]ollowing finite forms of verbs of motion, Slovene requires the use of a special infinitival form marked for intention, the supine“ (2006: 123). Hier führt Greenberg das neue Kriterium an, dass es sich um ein finites Verb der Bewegung handeln müsse. Dies ist allerdings nicht haltbar, denn das Korpus des modernen Slovenischen enthält etliche Beispiele für die Verbindung eines infiniten Verbes der Bewegung und Supinum. ${ }^{8}$

$$
\begin{aligned}
& \text { notwendig. }^{\text {ADV iti }} \text { gehen. }^{\text {VBINF }} \text { stimmen. }{ }^{\text {suP }} \text { gegen. }^{\text {PRÄ }} \\
& \text { 'Es ist notwendig, dagegen stimmen zu gehen.' } \\
& \text { (Gigafida, Übersetzung MJ) }
\end{aligned}
$$

Als spezielle Erscheinung der gesprochenen Standardsprache des Slovenischen führt Greenberg auch den verkürzten Infinitiv an, bei dem die Sprecher das $i$ der Infinitivendung

\footnotetext{
Abfrage: Infinitiv iti 'gehen' gefolgt von einem Supinum: (21.12.2016)

$\mathrm{http}: / / \mathrm{www}$.gigafida.net/Concordance/Search?mw=iti\&mst=All\&mpose=Exists\&mpose=Exists\&mpos=2\&m pos $=2 \&$ mposa $=3 \_n \& c e=E x i s t s \& c w=\& c s t=A l l \& c p o s e=E x i s t s \& c p o s=\& c p o s a=\& c p=I n t e r v a l \& c l p=3 \& c r p=3$ $\& \mathrm{ce} 1=$ Exists $\& \mathrm{cw} 1=\& \mathrm{cst} 1=\mathrm{All} \& \mathrm{cpose} 1=$ Exists\&cpose $1=$ Exists \&cpos $1=2 \& \operatorname{cpos} 1=2 \& \mathrm{cposa} 1=3 \_\mathrm{m} \& \mathrm{cp} 1=\mathrm{Int}$ erval\&clp $1=0 \& \operatorname{crp} 1=2$
} 
nicht mitsprechen, der Unterschied zum Supin liegt dabei im Akzent; Supinum: spât, verkürzter Infinitiv spàt 'schlafen' (2006: 61). Weitere Beispiele oder Erläuterungen zur Kasusrektion führt er hingegen nicht an.

\section{Fazit}

Zusammenfassend lässt sich konstatieren, dass die Herkunft der Autoren dahingehend eine Rolle spielt, als dass sie ihre heimatlichen Dialekte mit in die Grammatiken und Lehrbücher einfließen lassen. Dies betrifft insbesondere den Gebrauch des Supinums mit Genitiv und Akkusativ. So beschreiben die Gelehrten aus der Untersteiermark und aus Kärnten die Rektion des Supinums eher mit dem Genitiv, jene aus der Krain hingegen mit dem Akkusativ. Im späten 19. Jh., bzw. erstmals festgeschrieben in der Grammatik Brezniks von 1916, fand eine Angleichung dahingehend statt, dass zwar der Akkusativ vorherrscht, jedoch der Genitiv in verschiedenen Gegenden und Dialekten gemäß den Darstellungen noch anzutreffen ist. Dies ist im Wesentlichen der bis heute gültige Stand, der ein Ergebnis der Standardisierung des Slovenischen ist, die aber nicht die dialektalen Unterschiede beim Gebrauch des Supinums verschwinden ließ. Zudem ist auch evident, dass die jeweiligen Autoren Bezug auf andere Grammatiken oder aber Grammatiken anderer Sprachen nehmen. Die beschriebenen Veränderungen am Supinum sind allerdings progressiv und weisen keine „Rückschritte“ zurück zu einem restriktiveren Gebrauch, beispielsweise die Verwendung nur bei imperfektiven Verben auf. Dies ist auch anhand einer Korpusanalyse nachweisbar.

Anders verhält es sich mit der Entwicklung im Gebrauch des Supinums mit imperfektiven Verben. Die Überzeugung, wonach auch von perfektiven Verben das Supinum gebildet werden könne, entwickelt sich unabhängig von der Herkunft der jeweiligen Autoren und hat möglicherweise andere Ursachen.

Heutzutage entspricht auch die Verwendung von perfektiven Verben der Norm; im Gigafida-Korpus finden sich zahlreiche Beispiele dafür. Dennoch wären Untersuchungen notwendig, wie es zu dieser Gebrauchserweiterung kam, ob Einflüsse durch Sprachkontakte maßgeblich sind oder ob sie sprachökonomisch im Sinne einer Angleichung der Anwendungen von Infinitiv und Supinum mit Verben beiderlei Aspekts bedingt ist. Denkbar wäre zum Beispiel eine Veränderung der Anwendung des Supinums dergestalt, dass die einst beschriebene Beschränkung auf imperfektive Verben für die Sprechergemeinschaft nicht mehr nachvollziehbar bzw. nicht mehr sinnvoll erschien, was aber im Hinblick auf die sehr frühen Beispiele perfektiver und imperfektiver Verben im Supinum hinterfragt und anhand weiterer Daten ausgewertet werden sollten. Das slovenische Supinum hinterlässt eine Reihe offener linguistischer Fragen, denen sich weitere Untersuchungen widmen müssen.

\section{Abkürzungen}

$\begin{array}{llll}\text { INF } & \text { Infinitiv } & \text { VP } & \text { perfektives Verb } \\ \text { P } & \text { Perfekt } & \text { VB } & \text { Verb der Bewegung } \\ \text { PP } & \text { Personalpronomen } & \text { 1D ...3D } & 1 . / 2 . / 3 . \text { Person Dual } \\ \text { SUP } & \text { Supinum } & 1 \mathrm{P} \ldots 3 \mathrm{P} & 1 . / 2 . / 3 . \text { Person Plural } \\ \text { VI } & \text { imperfektives Verb } & 1 \mathrm{~S} \ldots 3 \mathrm{~S} & 1 . / 2 . / 3 . \text { Person Singular }\end{array}$




\section{Literatur}

Bajec, Anton, Kolarič, Rudolf, Rupel, Mirko \& Jakob Šolar (1956): Slovenska slovnica. Ljubljana.

Bohorič, Adam (1584): Arcticae horulae succisivae, de Latino Carniolana literatura, ad Latinae linguae analogiam accommodata, unde Moshoviticae, Rutenicae, Polonicae, Boëmicae \& Lusaticae lingvae, cum Dalmatica \& Croatica cognatio, facile deprehenditur. Wittenbergae.

Breznik, Anton (1916): Slovenska slovnica za srednje šole. Klagenfurt: Družbe sv. Mohorja.

Dajnko, Peter (1824): Lehrbuch der Windischen Sprache. Grätz: Kienreich.

Gigafida: Gigafida Korpus (http://www.gigafida.net). ${ }^{9}$

Greenberg, Marc L. (2006): Short Slovene Reference Grammar. Chapel Hill: SEELRC.

Grošelj, Robert (2014): The supine and the supine clause in South Slavic languages. Zeitschrift für Slawistik. 59/3. 301-324.

IMP: IMP Korpus starejša besedila (http://nl.ijs.si/imp/)

Janežič, Anton (1863): Slovenska slovnica s kratkim pregledom slovenskega slovstva ter z malim cirliskim in glagoliškim berilom za Slovence. Celovec.

Kopitar, Jernej (1809): Grammatik der Slavischen Sprache in Krain, Kärnten und Steyermark. Laibach: Korn.

Koupil, Karel (1947): Supinum. In: Naše řě 31. 78-79.

Levstik, Fran (1866): Die slovenische Sprache nach ihren Redetheilen. Laibach: Giontini.

Metelko, Franc Seraphin (1825): Lehrgebäude der slovenischen Sprache im Königreiche Illyrien und in den benachbarten Provinzen. Laibach.

Murko, Anton (1850): Theoretisch-practische Grammatik der Slowenischen Sprache in Steiermark, Kärnten, Krain und dem illyrischen Küstenlande. Zweite und sehr vermehrte Auflage. Grätz: Ferstl.

Murko, Anton (1843): Theoretisch-practische Grammatik der Slowenischen Sprache in Steiermark, Kärnten, Krain und dem illyrischen Küstenlande. Zweite und sehr vermehrte Auflage. Grätz: Ferstl.

Murko, Anton (1832): Theoretisch-praktische Slovenische Sprachlehre für Deutsche, nach den Volkssprecharten der Slovenen in Steiermark, Kärnten, Krain und Ungarns westlichen Distrikten. Grätz: Ferstl.

Sket, Jakob (1894): A. Janežičeva slovenska slovnica. Celovec: Družba sv. Mohorja.

Šmigoc, Janez Krstnik Leopold (1812): Theoretisch-praktische Windische Sprachlehre. Durch viele Uebungsstücke zum Uebersetzen mit einer auserlesenen Sammlung von Gesprächen und einem RadikalWörterbuche versehen herausgegeben von Johann Leopold Schmigoz. Grätz: Tusch.

Šuman, Josip \& Franc Miklošič (1881): Slovenska slovnica po Miklošičevi primerjalni. Ljubljana.

Svane, Gunnar Olaf (1958): Grammatik der slovenischen Schriftsprache. Kopenhagen: Rosenkilde und Bagger.

Toporišič, Jože (2004): Slovenska Slovnica. Maribor: Obzorja.

Toporišič, Jože (1984): Slovenska slovnica (2.), pregledana in razširjena izdaja ed. Maribor: Obzorja

Waldenfels, Ruprecht von (2012): The Grammaticalization of 'Give' + Infinitive. A Comparative Study of Russian, Polish, and Czech. Berlin, Boston: De Gruyter Mouton (= Trends in Linguistics, 256).

Vodnik, Valentin (1811): Pismenost ali gramatika sa perve shole. Ljubljana: Eger.

Zagajšek, Michael (1791): Slovennska Grammatika oder Georg Sellenko's Wendische Sprachlehre in deutsch und wendischen Vortrag mittels welcher Sowohl der Deutsche als auch der Wendische auf die leichteste Art diese Sprache regelrichtig zu reden und zu Schreiben von Selbsten erlernen kann. Zilli: Jenko.

\footnotetext{
9 Aufgrund einer Umstellung der slovenischen Korpora und deren Überführung sind die entsprechenden Internetadressen eventuell temporär oder dauerhaft nicht erreichbar.
} 



\title{
Continuatio, imitatio, renovatio? Die Verb-Subjekt-Linearisierung in Machiavellis Istorie fiorentine ${ }^{*}$
}

\author{
Laura Kersten (Erlangen)
}

\begin{abstract}
In Anlehnung an diachron ausgerichtete Untersuchungen zu Veränderungen der Textorganisation im Bereich der französischen Historiographie (Blumenthal 1990) soll anhand der Istorie fiorentine Niccolò Machiavellis der Frage nachgegangen werden, wie sich hier derartige Entwicklungen auf syntaktischer Ebene niederschlagen. Anhand von Verb-Subjekt-Strukturen soll exemplarisch illustriert werden, dass die sprachliche Organisation der Istorie fiorentine vor dem Hintergrund ihrer Situierung im Kontext der Historiographie im 16. Jahrhundert Ansätze für eine differenzierte Analyse scheinbar kanonischer Formen und Funktionen der Verb-SubjektSerialisierung bietet. Angesichts einer gewissen Kontinuität in Bezug auf die funktionale Auslastung der Verb-Subjekt-Linearisierung liegt das Augenmerk dabei auf der Beziehung zwischen kanonischen und 'randständigeren' Okkurrenzen.
\end{abstract}

\section{Einleitung}

In einer diachron ausgerichteten Untersuchung zur französischen Historiographie illustriert Peter Blumenthal, wie das Zusammenspiel kultur- und sozialgeschichtlicher (vgl. Blumenthal 1990: 57) sowie sprachsysteminhärenter Faktoren die Gestalt von Texten beeinflusst und vor allem wie sich dort das Verhältnis von Informativität und Verständlichkeit ${ }^{1}$ in je verschiedenartiger Weise darstellt (vgl. Blumenthal 1990: 25). Zur Skizzierung der ausdruckstrukturelltextuellen Manifestation dieser Veränderungen bedient sich Blumenthal methodisch des Vergleichs von Kohäsionsmustern, um auch makrostrukturelle Aspekte in den Blick nehmen zu können (Blumenthal 1990: 26-27). Was bisher, auch jenseits dieser Untersuchungen und speziell mit Blick auf das Italienische, noch relativ unbeachtet geblieben zu sein scheint, ist die Frage nach dem textpragmatischen Funktionieren von Texten und der Rolle der Textpragmatik bei einer historischen Rekontextualisierung des Textualitätskonzepts. Denn hat man zwar im Allgemeinen ein ausgeprägtes Bewusstsein dafür, dass Texte im Sinne kommunikativer (Handlungs-)Muster stets vor dem Hintergrund ihrer Genese und diskurstraditionellen

\footnotetext{
Ich danke den GutachterInnen für ihre hilfreichen Hinweise zu einer früheren Fassung dieses Beitrags.

Blumenthal (1990: 25) bedient sich des etwas schwer zu operationalisierenden Begriffs der Textleistung, welche er als Produkt aus „Informativität (Dichte der aufgeführten neuen Sachverhalte und der sprachlich dargestellten Zusammenhänge zwischen ihnen) und der Eingängigkeit (Verständlichkeit und Behaltbarkeit) des Textes“ definiert. Die Beziehung zwischen der Textleistung und den Kohäsionsmustern sieht er darin gegeben, dass durch die Form und das Maß der Variation bei der Wahl der Kohäsionsmittel die Taktung, in der die Vergabe von Informationen erfolgt, modifiziert wird, was auch eine Veränderung bei der kommunikativen Funktionalisierung der Inhalte bewirken kann (vgl. 1990: 29).
} 
Entwicklung zu betrachten sind, ${ }^{2}$ so ist doch noch nicht im Detail geklärt, was Textualität zu einer bestimmten Epoche ausmacht und wie sich die Funktion der bei der Verschriftlichung (Oesterreicher 1993) zur Verfügung stehenden sprachlichen Mittel im Verlauf der Zeit verändert.

Ziel des vorliegenden Beitrags kann es nicht sein, diese Fragen umfänglich zu beantworten. Vielmehr soll anhand von Verb-Subjekt-Strukturen mit potentiell thematischem Subjekt als einem ausgewählten Phänomen aufgezeigt werden, wie diese in einem konkreten Text, den Istorie fiorentine von Niccolò Machiavelli, hinsichtlich ihres Beitrags zur Textstrukturierung funktionalisiert sind. ${ }^{3}$ Mit der Analyse der VS-Strukturen soll auch eine Methode vorgestellt werden, mit der man die Form der Kodierung pragmatischer Relationen in Texten flankierend zur traditionellen Modellierung von Kohäsion und Kohärenz untersuchen kann. Dabei sollen gerade saliente Verwendungskontexte, die hinsichtlich ihrer Auftretenssystematik und Normverankerung noch genauer zu untersuchen sind, herangezogen werden, um neue Perspektiven auf mögliche Faktoren zu entwickeln, die langfristig für das Verschwinden textstrukturierender VS-Linearisierungen im Italienischen (vgl. Blumenthal 1980) verantwortlich sind.

Zur Einführung des terminologisch-methodischen Rahmens soll im Folgenden zunächst anhand von Belegen aus den Istorie das funktionale Spektrum der VS-Strukturen kurz umrissen werden, bevor die Frage nach der Funktionalisierung von VS-Serialisierungen mit potentiell thematischem Subjekt erörtert wird. Dabei gilt es zum einen zu zeigen, dass textstrukturierende VS-Linearisierungen des Altitalienischen fortgeführt werden und nicht nur als formale Imitation in den Text Eingang gefunden haben, und zum anderen, auf neue oder zumindest divergierende Entwicklungen aufmerksam zu machen. Hervorzuheben ist hierbei der exemplarische Charakter der Analyse.

\section{Text und Untersuchungsgegenstand}

Die Istorie fiorentine, eine Auftragsarbeit für Papst Clemens VII. (Giulio de' Medici), stellen sich in die Tradition der humanistischen Geschichtsschreibung. ${ }^{4} \mathrm{Da}$ über die textsortenlinguistische Klassifikation von 'Geschichtsschreibung' bisher noch keine Einigkeit herrscht (vgl. Blumenthal 2000), soll als vages Suchraster für textsortentypische Merkmale die von Linguisten (vgl. Ferranti 2010; Colussi 2014) wie Geschichtswissenschaftlern gleichermaßen angeführte „Doppelcodierung des historischen Diskurses als Erzählung und Argumentation“ (Harth 1996: 840) zugrunde gelegt werden.

Ferner ist zu berücksichtigen, dass es sich bei den Istorie fiorentine um einen historiographischen Text in der Volkssprache, dem Florentinischen, handelt (vgl. dazu Franceschini 2011: 845), der sich somit möglicherweise auch ein Stück weit von der lateinischen historiographischen Tradition absetzt und sich eventuell zweier Traditionslinien bedient. Konkret heißt dies, dass v.a. im syntaktischen Bereich Hinweise zu suchen sind, ob z.B. syntaktische Muster auf lateinische Vorbilder zurückgehen oder aus altitalienischen Quellen übernommen bzw. in vergleichbaren Kontexten aus stilistischen Gründen nachgebildet worden sind. Da

2 In jüngerer Zeit hat z. B. Schrott (2015: 479-483) mit Blick auf den Aspekt der „Nähe und Lebendigkeit in privaten Briefen" darauf hingewiesen, dass in Texten in Abhängigkeit von den verfolgten kommunikationsstrategischen Zielen verschiedene Diskurstraditionen einfließen können.

3 Im Altitalienischen ist der Einsatz von VS-Linearisierungen mit thematischem Subjekt zur Strukturierung von Texten gut dokumentiert (vgl. u.a. Blumenthal 1980; Fesenmeier 2004).

4 Vgl. zur florentinischen Geschichtsschreibung Matucci (1991). 
gerade für die Satzgliedstellung des Öfteren das Argument eines latinisierenden Stils ins Feld geführt wurde (vgl. Renzi 2008: 2836), nicht aber die Hypothese des funktionalen Einsatzes bestimmter Muster untersucht worden ist, erscheint es lohnenswert, alternative Erklärungsoptionen zu prüfen.

Gegenstand der Untersuchung sind dabei, wie bereits oben erläutert, VS-Linearisierungen mit potentiell thematischem Subjektreferenten im deklarativen Hauptsatz. Subordinierte Sätze bleiben von der Betrachtung ausgenommen, da hier andere Regularitäten bei der syntaktischen Gestaltung angenommen werden müssen.

\section{VS-Strukturen in den Istorie fiorentine}

Die Forschung hat sich bisher mit der Satzgliedstellung in den Istorie fiorentine kaum befasst. Obwohl Renzi (2008: 2836) andeutet, Machiavelli entziehe sich als einer der wenigen Autoren des Cinquecento im Bereich der Satzgliedstellung dem Bemboschen Normierungsdruck, existieren dazu keine einschlägigen Studien. Einzig Ferranti (2010) verweist in ihrer Dissertation zu Aspetti della sintassi e della testualità della storiografia toscana cinquecentesca auf die Existenz von VS-Serialisierungen in den Istorie fiorentine. Aufgrund der deskriptiven Ausrichtung der Arbeit in diesem Punkt liefern aber die Ausführungen mit Blick auf den vorliegenden Beitrag keine 'neuen' Einsichten.

\subsection{Typen von VS-Serialisierungen: thetisch versus kategorisch}

Es soll im Folgenden zunächst die Unterscheidung zwischen zwei unterschiedlichen kommunikativen Profilen, thetisch und kategorisch, erläutert werden (vgl. Sasse 1995). ${ }^{6}$ Hierzu die Belege (1a) und (1b) aus den Istorie fiorentine:

(1) a. A costoro fu mostro [...] che, se venivono nella città secretamente, gli riceverebbono in casa, donde e' potevono poi, uscendo, ammazzare messer Maso degli Albizzi [...]. Mossi per tanto costoro da queste speranze, a dì 4 di agosto nel 1397, vennono in Firenze, ed entrati secretamente dove era stato loro ordinato, mandorono ad osservare messer Maso, volendo da la sua morte muovere il tumulto. Uscì messer Maso di casa, e in uno speziale, a San Piero Maggiore propinquo, si fermò. (Istorie fiorentine III, 27) ${ }^{7}$

'Ihnen kam [...] die Weisung zu, dass sie ihnen Aufnahme in ihrer Wohnung gewähren würden, wenn sie heimlich in die Stadt kämen; von dort aus könnten sie sodann Messer Maso degli Albizzi ermorden [...].Von dieser Aussicht angelockt kamen die Genannten am 4. August 1397 nach Florenz, und nachdem sie heimlich in die bezeichneten Wohnungen sich begeben, ließen sie Messer Maso beobachten, dessen Mord das Signal

5 Mit der Subjekt-Inversion im Principe hat sich Cernecca (1982) befasst. Durch sein Plädoyer für stilistische Faktoren bei der Wahl des Serialisierungsmusters und vor allem durch die Heterogenität der Belege (hier werden sowohl Nebensätze als auch pronominal realisierte Subjektreferenten gleichermaßen behandelt) ist die Arbeit für eine textpragmatisch motivierte Untersuchung von eingeschränkter Relevanz.

6 „Kommunikatives Profil“ bezieht sich auf die pragmatische Gliederung einer Äußerung und ist von deren semantisch-propositionaler Struktur zu unterscheiden (vgl. Oesterreicher 1991). Diese Unterscheidung ist dadurch motiviert, dass es sich bei den untersuchten Sätzen um in einem konkreten Kontext funktionierende Äußerungen handelt (Stark 1997: 2-3).

7 Die Istorie fiorentine werden zitiert nach der Ausgabe Machiavelli (2010). 
zum Aufstand sein sollte. Messer Maso verließ das Haus und verweilte im Laden eines in der Nähe von San Pier maggiore wohnenden Apothekers. ${ }^{, 7}$

b. Onde che Zenone [...] concesse a Teoderigo il venire contro a quello [Odeacre] e pigliare la possessione di Italia. Il quale subito partì di Pannonia [...]; e venuto in Italia, ammazzò Odeacre e il figliuolo, e con l'esemplo di quello, prese il titulo di re di Italia; e pose la sua sedia in Ravenna, mosso da quelle cagioni che feciono già a Valentiniano imperadore abitarvi. Fu Teoderigo uomo nella guerra e nella pace eccellentissimo, donde nell'una fu sempre vincitore, nell'altra benificò grandemente le città e i popoli suoi. (Istorie fiorentine I, 4)

'Daher erlaubte Zeno dem Theodorich, gegen diesen zu ziehen und von Italien Besitz zu nehmen. Da brach Theodorich sogleich aus Pannonien auf [...]; er zog nach Italien, tötete den Odoaker und dessen Sohn, nahm nach dessen Beispiel den Titel eines Königs von Italien an und wählte zu seinem Sitz Ravenna [...] aus denselben Gründen, die einst Valentinian bewogen hatten, dort zu wohnen. Theodorich war ein im Kriege wie im Frieden ausgezeichneter Mann: im Kriege immer glücklich, war er im Frieden der Wohltäter seiner Völker und Städte.'

In (1a) lässt sich in Bezug auf den referentiellen Status des Denotats des Verbs und des Subjektreferenten konstatieren, dass einzig der Subjektreferent messer Maso im Vorsatz vorerwähnt und somit bekannt bzw. im Sinne von Chafe (1976: 36-41) identifizierbar ist. Identifizierbarkeit ist mit Blick auf die potentielle Thematizität des Referenten im Satz als zentrales Kriterium zu betrachten. Sie wird hier den Kriterien '(aus dem situativen Kontext) bekannt' bzw. '(im sprachlichen Kontext) gegeben' vorgezogen, da identifizierbare Referenten nicht notwendigerweise bekannt oder gegeben sein müssen (vgl. Fesenmeier 2003: 30). Dass allerdings eine thematische Interpretation des Subjektreferenten in (1a) inadäquat ist, offenbart der Ko-Text: Gegenstand des Absatzes ist eine Verschwörung gegen messer Maso. Hierbei werden zunächst die Rahmenbedingungen zur Umsetzung des Plans aus der Sicht der Verschwörer referiert (mandorono ad osservare messer Maso 'ließen sie Messer Maso beobachten'). Dann erfolgt ein abrupter Übergang zur Darstellung der quasi simultan einsetzenden Handlung, d.h. dem Verlassen des Hauses durch messer Maso, welche in der Äußerung mit einer VS-Struktur ausgedrückt wird. Die VS-Struktur fungiert als Mittel, um die pragmatische Gliederung der Äußerung den ko-textuellen Gegebenheiten anzupassen: Angesichts des Übergangs von der Darstellung der Überwachungsabsicht zur Überwachungssituation, wie man das Verhältnis der Propositionen zueinander bezeichnen kann, wird mit Blick auf die Erwartungshaltung des Rezipienten unwahrscheinlich, dass pragmatisch gesehen mit Usci messer Maso di casa 'Messer Maso verließ das Haus' ein Aussage über den Subjektreferenten erfolgen soll, obwohl messer Maso vorerwähnt ist: Der Subjektreferent messer Maso erscheint eingebettet in einen gänzlich anderen Handlungszusammenhang, sodass der Rezipient keinen Grund zur Annahme hat, nun etwas über messer Maso zu erfahren; relevant ist vielmehr das Ereignis selbst, in dem messer Maso natürlich eine zentrale Rolle zukommt. Um Rückschlüsse auf die pragmatische Gliederung einer Äußerung ziehen zu können (vgl. Daneš 1964; Oesterreicher 1991: 357), ist die Funktion der Äußerung also immer im Textzusammenhang zu betrachten, d.h. ausschlaggebend sind die Intention des Produzenten und die Erwartungshaltung des Rezipienten in Bezug auf den kommunikativen Wert der Äußerung (Chafe 1976: 36-41). Liegt also in (1a) zwar auf semantisch-propositionaler Ebene eine Aussage über messer Maso vor, so ist auf der hier relevanten pragmatischen Ebene einzig das Kriterium der pragmatic aboutness von Bedeutung (Reinhart 1981): „We [...] intend in

\footnotetext{
Alle Übersetzungen der Istorie fiorentine (teilweise adaptiert) nach Machiavelli (1934).
} 
general to give or add information about what is a matter of standing or current interest or concern" (Strawson 1964: 115). Ob das Kriterium der pragmatic aboutness greift, lässt sich durch die Ermittlung der im Kontext adäquaten underlying question (Hatcher 1956) feststellen: Die Frage 'Was geschah dann?' bzw. 'Was geschah weiter?' ist offensichtlich textpragmatisch adäquater als 'Was geschah dann mit messer Maso?', d.h. es geht um eine globale, eventive Perspektivierung eines Sachverhalts - an dem natürlich messer Maso beteiligt ist. Für die pragmatische Gliederung der vorliegenden Äußerung bedeutet dies, dass sie nicht als in Thema und Rhema analysierbar (Sasse 1987: 558), sondern als ,globalrhematisch“ erscheint. Nach Sasse (1995) sollen hier Äußerungen mit einem derartigen informationsstrukturellen Profil als thetisch bezeichnet werden. ${ }^{8}$ Nicht der 'gegebene' Status des Subjektreferenten, sondern die Art und Weise der Perspektivierung der Sachverhaltsdarstellung ist hier ausschlaggebend dafür, dass man nicht mit einer pragmatisch binären, in Thema und Rhema gliederbaren, also nach Sasse (vgl. 1987: 558) kategorischen Äußerung befasst ist. Dabei dient die - auch syntaktisch markierte - VS-Struktur der Blockierung einer thematischen Lesart des Subjektreferenten, die sich bei der unmarkierten Satzgliedstellung SV einstellen würde.

Umgekehrt behindert die VS-Linearisierung nicht zwangsläufig die Themafunktion des Subjektreferenten, sodass auch eine informationsstrukturell kategorische Äußerung vorliegen kann: In (1b) antwortet Fu Teoderigo... 'Theodorich war...' nur auf die Frage 'Wie war Teoderigo als Mensch?', da diese Äußerung an den Bericht über seine Taten anschließt. Folglich kann man nicht auf Basis der Reihenfolge der Konstituenten auf ein bestimmtes Profil schließen. Als weiteres Argument für den Themastatus von Teodorigo lässt sich anführen, dass eine nominale Explizierung für die Sicherstellung des Textverständnisses nicht erforderlich gewesen wäre. Wie im Altitalienischen, wo VS zur „Kodierung unbetont thematischer Subjekte“ (Selig 1992: 208) dienen konnte, wird auch hier der Subjektreferent in einer kommunikativ weniger salienten, also unbetonten Position kodiert.

Die Gegenüberstellung der Beispiele (1a) und (1b) zeigt also, dass die Unterscheidung in 'thetisch' und 'kategorisch' für eine ko- und kontextsensible Bestimmung der pragmatischen Gliederung von Äußerungen nutzbar gemacht werden kann, denn die Identifizierbarkeit eines Referenten (vgl. v.a. (1a)) stellt kein hinreichendes Kriterium dar, um daraus auf die Funktion eines Referenten bzw. einer Konstituente als (pragmatisches) Thema schließen zu können. Anhand der Belege (1a) und (1b) ist ersichtlich geworden, dass in den Istorie fiorentine VSStrukturen vorhanden sind, die pragmatisch gesehen sowohl thetischen als auch kategorischen Äußerungen entsprechen.

\subsection{Continuatio versus imitatio}

Konnte mit den Beispielen (1a) und (1b) also auch illustriert werden, dass die Istorie fiorentine in der Tat VS-Serialisierungen beider Typen, thetisch und kategorisch, enthalten, so wirft (1b) dennoch die Frage auf, ob es sich hierbei auch um eine funktionale Fortsetzung der altitalienischen VS-Strukturen mit thematischem Subjekt handelt oder um eine rein formale Imitation, die den spezifischen ko- und kontextuellen Auftretensbedingungen in der älteren Sprachstufe enthoben ist. Zur Beantwortung der Frage nach der 'Produktivität' dieser Struktur im Cinquecento bietet sich zunächst der Vergleich einer Passage aus der Cronica delle cose

8 Zur sprachphilosophischen Fundierung des Konzepte 'Thetiziät' und 'Kategorizität' und den verschiedenen thetischen Funktionen im Gegenwartsitalienischen siehe u.a. Ulrich (1985), Sasse (1995) und Venier (2002: 15-26). Zu den Funktionen von Thetizität im Altitalienischen siehe Fesenmeier (2003). 
occorenti ne' tempi suoi (wohl 1310-1312) von Dino Compagni mit einem Abschnitt aus den Storie fiorentine (1508/1509) von Francesco Guicciardini an:

(2) a. Finito l' uficio di detto messer Andrea, la parte bianca [...] chiamarono messer Schiatta Amati, de' Cancellieri bianchi, per loro capitano di guerra; e dieronli tanta balìa, che i soldati rispondeano a lui, mandava i bandi da sua parte, e pene imponea, e cavalcate contra i nimici, sanza alcuno consiglio. Era il detto cavaliere uomo molto piatoso e temeroso, la guerra non li piacea, e tutto era contrario al suo consorto, messer Simone da Pantano de' Cancellieri neri (Compagni, Cronica delle cose occorenti ne' tempi suoi, I, 27; zit. n. TLIO, Ed. Cappi)

'Das Amt des Messer Andrea war zu Ende, die Weißen aber [...], weil sie keinen Anführer hatten, beriefen Messer Schiatta Amati von den weißen Cancellieri als ihren Kriegsobersten und gaben ihm solche Vollmacht, dass die Söldner ihm ganz unterstellt waren, und er gab in seinem Namen Gesetze und verhängte Strafen und sandte Reiterscharen gegen die Feinde, ohne dass Rat gehalten wurde. Es war aber besagter Ritter ein weichmütiger Mann und sehr furchtsam; der Krieg gefiel ihm nicht, und er war das Widerspiel von seinem Verwandten, Messer Simone da Pontano von den schwarzen Cancellieri. ${ }^{9}$

b. [...] finalmente a dì. .. di aprile 1492 [Lorenzo de' Medici] passò della presente vita. [...] Era Lorenzo de' Medici di età di anni quarantatré quando morì, ed era stato al governo della città ventitré anni [...]. (Guicciardini, Storie fiorentine, IX, 2f; zit. n. LIZ) '[...] schließlich schied [Lorenzo de Medici] am ... April 1492 aus dem Leben. [...] Lorenzo de' Medici war 43 Jahre alt, als er starb, und hatte 23 Jahre lang die Führung der Stadt inne gehabt [...].' (Übersetzung L.K.)

Die Beispiele (2a) und (2b) weisen offensichtliche Parallelen zu dem Beleg in (1b) auf: In allen drei Fällen werden zunächst Ereignisse referiert, die jeweils Anlass dazu geben, dass eine nähere Charakterisierung der Schlüsselpersönlichkeiten, die ihrerseits jeweils DiskursTopic des Abschnitts sind, erfolgt. Auch die Anwendung des underlying question-Tests 'Wie war der besagte Cavaliere?' bzw. 'Wie alt war Lorenz de' Medici, als er starb?' - zeigt, dass die Referenten des postverbalen Subjekts Themastatus haben. Die oben aufgeworfene Frage nach der Produktivität lässt sich also affirmativ beantworten, und zwar in sowohl formaler wie funktionaler Hinsicht: Der Grund für den Rekurs auf VS ist im Beitrag zur Textgliederung zu suchen, nämlich im Wechsel von der Ebene der Narration zur Ebene der Charakterisierung. Es gibt also sowohl in Machiavellis Istorie fiorentine als auch in Guicciardinis Storie fiorentine Evidenz dafür, dass VS-Strukturen mit thematischem Subjekt weiterhin in textstrukturierender Funktion angewendet werden (vgl. dazu auch Blumenthal 1980).

Hat dieser kurze Vergleich der Cronica delle cose occorenti ne' tempi suoi mit den Istorie fiorentine und den Storie fiorentine zwar zeigen können, dass VS-Strukturen nach wie vor zur Strukturierung von Texten verwendet werden bzw. Textstrukturierung durch syntaktische Mittel erreicht wird, so findet man jedoch in den Istorie fiorentine Machiavellis neben kanonischen Belegen wie (1b) auch solche, die sich dem ersten Anschein nach ein Stück weit von der Tradition entfernen.

Solche Fälle liegen - in je unterschiedlicher Ausprägung - in den Belegen (3a) und (3b) vor, in denen den Herrscherfiguren Federigo und Clefi bestimmte Charaktereigenschaften zugewiesen werden:

9 Übersetzung (teilweise adaptiert) nach Compagni (1914). 
(3) a. Dopo la morte di Pascale e di Errico IV, seguirono più papi e più imperadori, tanto che il papato pervenne ad Alessandro III, e lo Imperio a Federigo Svevo, detto Barbarossa. Avevano avuto i pontefici, in quelli tempi, con il popolo romano e con gli imperadori molte difficultà, le quali al tempo del Barbarossa assai crebbero. Era Federigo uomo eccellente nella guerra, ma pieno di tanta superbia che non poteva sopportare di avere a cedere al Pontefice; nondimeno nella sua elezione venne a Roma per la corona, e pacificamente si tornò nella Magna. Ma poco stette in questa opinione, perché tornò in Italia per domare alcune terre in Lombardia [...]. (Istorie fiorentine I, 18)

'Nach Paschalis' und Heinrichs IV. Tod folgten mehrere Päpste und Kaiser, bis das Papsttum an Alexander III. gelangte, die Kaiserwürde an Friedrich Herzog von Schwaben, genannt Barbarossa. Die Päpste hatten in jener Zeit viele Schwierigkeiten mit dem römischen Volk und mit den Kaisern gehabt, die zur Zeit des Barbarossa noch größer wurden. Friedrich war ein tapferer Kriegsmann, aber so voll Hochmut, dass er den Gedanken, dem Papste nachzustehen, nicht zu ertragen vermochte. Nichtsdestoweniger kam er zu seiner Wahl nach Rom, die Krone zu empfangen, und kehrte friedsam nach Deutschland zurück. Dies währte aber nicht lange: denn er unternahm einen zweiten Zug nach Italien, um einige ungehorsame Städte in der Lombardei zu züchtigen, [...].'

b. I Longobardi intanto [...] feciono Clefi loro re; il quale riedificò Imola, stata rovinata da Narsete, occupò Rimino e, infino a Roma, quasi ogni luogo; ma nel corso delle sue vittorie morì. Questo Clefi fu in modo crudele, non solo contro agli esterni, ma ancora contro ai suoi Longobardi, che quegli, sbigottiti della potestà regia, non vollono rifare più re; ma feciono intra loro trenta duchi, che governassero gli altri. (Istorie fiorentine I, 8)

'Die Longobarden machten unterdessen den Klef zu ihrem König, welcher das von Narses zerstörte Imola wieder aufbaute, von Rimini bis Rom hin fast jeden Ort besetzte, aber im Lauf seiner Siege starb. Dieser Klef war in einer Weise grausam nicht nur gegenüber den Fremden, sondern auch gegen seine Langobarden, dass diese keinen König mehr wählen wollten und dreißig Herzogen die Regierung übertrugen.'

In (3a) steht außer Zweifel, dass der Subjektreferent Federigo aufgrund seiner Vorerwähntheit identifizierbar und auch Thema der fraglichen Äußerung ist. Auffällig ist allerdings, dass der Subjektreferent Federigo beim Leser also zwar 'aktiviert' ist, jedoch nicht das DiskursTopic darstellt. Der in Frage stehenden Äußerung geht nämlich eine rahmende Darstellung der allgemeinen Herrschaftssituation voraus, innerhalb derer zu erwarten wäre, dass Gegenstand der folgenden Ausführungen die Darstellung der Problematik dezidiert aus der Sicht des Klerus sein würde. Diese Erwartung inhaltlicher Kontinuität wird allerdings nicht erfüllt, vielmehr folgt unmittelbar die Charakterisierung einer - im Unterschied zu (1b), (2a) und (2b) bisher ausdrucksstrukturell nur marginal profilierten Entität: Federigo.

In (3b) sind im Grunde dieselben Prämissen mit Blick auf den Themastatus des Subjektreferenten Clefi und den propositionalen Gehalt der Äußerung erfüllt, sodass man aufgrund der Wahl der Serialisierung SV zunächst einmal eine 'Anomalie' vermuten würde, zumal hier scheinbar ein Übergang von der Narration der Ereignisse zur Schilderung der Eigenschaften des Subjektreferenten Clefi erfolgt, also analog zu (2a), (2b) und (3a). Betrachtet man jedoch die ko-textuelle Einbettung der Äußerung genauer, zeigt sich hier ein wesentlicher Unterschied, der (3b) nicht nur von (2a) und (2b) abgrenzt - Clefi ist kein Diskurs-Topic -, sondern auch von (3a): Nachdem der Subjektreferent in Questo Clefi fu in modo crudele... 'Dieser Klef war in einer Weise grausam...' charakterisiert worden ist, geht es im weiteren Verlauf der Äußerung nicht mehr um Clefi an sich, sondern vielmehr um Clefis Verhalten als Ursache für die Aversion der Langobarden gegen die Monarchie. Durch den Rekurs auf SV wird signalisiert, dass der Subjektreferent in einen neuen, anderen Argumentationszusammenhang eingeordnet wird. 
Wie in Kap. 3.1 bereits erläutert, ist also auch in (3a) und (3b) vom dargelegten Sachverhalt kein unmittelbarer Rückschluss auf die textstrukturelle Funktion der Äußerung möglich und auch zwischen dem Serialisierungsmuster (VS bzw. SV) und der Eröffnung eines neuen Argumentationszusammenhangs besteht kein gerichteter Zusammenhang. Nimmt man zusätzlich Beleg (1a) mit in die Betrachtung, zeigt sich zudem, dass das Linearisierungsmuster VS polyfunktional ist: In (1a) bestand der Grund für den Rekurs auf VS darin, dass der Subjektreferent messer Maso in einen neuen Handlungszusammenhang eingebettet wird und diese Einbettung eine kategorische Profilierung der Äußerung kontextuell unangemessen erscheinen lässt. In (3b) hingegen muss wegen der Integration von Clefi in einen neuen Argumentationszusammenhang die Serialisierung SV gewählt werden, damit die fragliche Passage nicht als Ausscheren aus der Narration und somit als Übergang zur Charakterisierung aufgefasst wird, wie es bei VS der Fall wäre. In (3b) offenbart also der Ko-Text, dass es nicht das kommunikative Ziel ist, Hintergrundinformationen zu einem bekannten Referenten zu vermitteln, wie es in (1b), (2a), (2b) und (3a) der Fall war, sondern dass der Referent als Thema in einen neuen thematischen Zusammenhang gesetzt wird. Ähnliche ko-textuelle Konfigurationen finden sich auch im Altitalienischen; man betrachte hierzu Beleg (4) aus der Nuova Cronica von Giovanni Villani: ${ }^{10}$
Il quarto confine di Toscana di verso settentrione sono le dette alpi Apennine, le quali confinano e partono la provincia di Toscana da Lombardia e Bologna e parte di Romagna; e gira la detta provincia di Toscana VII.c miglia. Questa provincia di Toscana ha più fiumi: intra gli altri reale e maggiore si è il nostro fiume d'Arno [...]. (Villani, Nuova cronica, II, 6; zit. n. TLIO, Ed. Porta)
'Die vierte Grenze der Toskana gegen Norden bilden die bereits genannten Apenninen, die die Provinz Toskana von der Lombardei, Bologna und einem Teil der Romagna abgrenzen; die besagte Provinz Toskana misst im Umfang 700 Meilen. In dieser Provinz Toskana gibt es mehrere Flüsse: in mitten der anderen königlich und als mächtigster befindet sich unser Fluss Arno [...].' (Übersetzung L.K.)

Der Abschnitt befasst sich mit den geographischen Eigenschaften der provincia di Toscana. In Il quarto confine di Toscana... 'Die vierte Grenze der Toskana...' und Questa provincia di Toscana... 'In dieser Provinz Toskana...' werden dabei je die Sub-Topics 'Grenzen' und 'Hydrographie' behandelt. Betrachtet man die ko-textuelle Einbettung von gira la detta provincia di Toscana VII.c miglia 'die besagte Provinz Toskana misst im Umfang 700 Meilen' (VS), wird deutlich, dass hierin nach der Beschreibung der Grenzen abschließend noch die konkrete Ausdehnung der provincia 'Provinz' expliziert wird. Dadurch, dass inhaltliche Kontinuität in Bezug auf das Sub-Topic 'Grenzen' gegeben ist, wird der thematische und ausdrücklich als gegeben markierte Subjektreferent in der kommunikativ nicht prominenten Position hinter dem Verb integriert. Inhaltliche Diskontinuität wird hingegen mit SV signalisiert: Mit Questa provincia di Toscana 'In dieser Provinz Toskana' wird ein neues Sub-Topic, 'Hydrographie', eingeleitet und dabei die kommunikativ prominente Position mit dem Subjektreferenten (als volle NP realisiert) besetzt, sodass die provincia di Toscana 'Provinz Toskana' explizit als Thema gesetzt erscheint, allerdings in einem neuen inhaltlichen Zusammenhang.

Zusammenfassend lässt sich zunächst festhalten, dass Machiavelli VS-Strukturen in durchaus traditionell-funktionaler Weise einsetzt (1b), sie aber auch in Kontexten verwendet, die nicht von der Tradition gedeckt sind (3a). Umgekehrt führt ein im Prinzip geeigneter Kontext

10 Zum folgenden Beispiel vgl. Fesenmeier (2004: 106-107). 
nicht dazu, dass automatisch auf diese Serialisierung rekurriert wird (3b). Machiavelli erscheint also nicht einfach 'blind' der imitatio verpflichtet, sondern grundsätzlich der continuatio, was freilich Ansätze eines kreativen Umgangs mit syntaktischen Mustern zur Textstrukturierung nicht ausschließt.

\subsection{Innovatio/renovatio?}

Jenseits der bisher diskutierten Beispiele lassen sich jedoch noch weitere Belege finden, in denen die ko-textuelle Einbettung der Äußerung in Verbindung mit dem referentiellen Status des jeweiligen Subjektreferenten den Anschein erweckt, dass man mit einer Innovation bzw. einer 'Erneuerung' in den Bahnen des 'Alten', also mit einer renovatio, befasst ist. Inwieweit dabei eher die stilistisch-rhetorischen und textpragmatischen 'Bahnen' der lateinischen bzw. volkssprachlichen Historiographie (bzw. allgemeiner Textualität) den Ausgangspunkt bilden, muss an dieser Stelle freilich noch offen bleiben.

Eine vor diesem Hintergrund relevante Auffälligkeit bildet die nicht selten anzutreffende extreme Distanzstellung von Auxiliar und Partizip (vgl. Scavuzzo 2003: 143), wie sie in (5a) vorliegt.

(5) a. E benché il Papa fusse da molti avvertito dello inganno, nol credeva, né poteva udire alcuno che dicesse il contrario. Era la città di Ravenna da Ostasio da Polenta per la Chiesa governata. Niccolò, parendogli tempo da non differire più la impresa sua, perché Francesco suo figliuolo aveva, con ignominia del Papa, saccheggiato Spuleto, deliberò di assaltare Ravenna, o perché giudicasse quella impresa più facile, o perché gli avessi con Ostasio secretamente intelligenzia; e in pochi giorni, poi che l'ebbe assalita, per accordo la prese. (Istorie fiorentine $\mathrm{V}, 17$ )

'Und obgleich man den Papst vor Trug warnte, glaubte er es nicht und wollte doch nichts davon hören. Es wurde die Stadt Ravenna von Ostasio da Polenta für den römischen Stuhl verwaltet. Da es nun dem Niccolò Zeit schien, den Angriff nicht aufzuschieben, weil sein Sohn Francesco zu des Papstes Schaden Spoleto gebrandschatzt hatte, so beschloss er gen Ravenna zu ziehen, entweder weil dies Unternehmen ihm leichter vorkam oder weil er mit Ostasio im Einverständnisse war.'

b. E benché il Papa fusse da molti avvertito dello inganno, nol credeva, né poteva udire alcuno che dicesse il contrario. La città di Ravenna era da Ostasio da Polenta per la Chiesa governata. Niccolò, parendogli tempo da non differire più la impresa sua, perché Francesco suo figliuolo aveva, con ignominia del Papa, saccheggiato Spuleto, deliberò di assaltare Ravenna, o perché giudicasse quella impresa più facile, o perché gli avessi con Ostasio secretamente intelligenzia; e in pochi giorni, poi che l'ebbe assalita, per accordo la prese.

'Und obgleich man den Papst vor Trug warnte, glaubte er es nicht und wollte doch nichts davon hören. Die Stadt Ravenna wurde von Ostasio da Polenta für den römischen Stuhl verwaltet. Da es nun dem Niccolò Zeit schien, den Angriff nicht aufzuschieben, weil sein Sohn Francesco zu des Papstes Schaden Spoleto gebrandschatzt hatte, so beschloss er gen Ravenna zu ziehen, entweder weil dies Unternehmen ihm leichter vorkam oder weil er mit Ostasio im Einverständnisse war.'

Ohne Ko-Text-Kenntnis würde man annehmen, es handelt sich bei (5a) um Hintergrundinformationen - hier die Herrschaftssituation - zu einem bereits als Thema etablierten Subjektreferenten. Wenn man aber den Textzusammenhang berücksichtigt, erscheint dies einigermaßen 
unplausibel, da Ravenna nur einmal en passant (Camurata, luogo intra Furlì e Ravenna 'Camurata, Ort zwischen Forlì und Ravenna') mehrere Zeilen zuvor kurz erwähnt worden war. Dem Leser wird hier also vielmehr ein globaler Sachverhalt, bestimmte machtpolitische Gegebenheiten, vorgelegt, dessen Relevanz für das nachfolgend dargestellte Geschehen erst später ersichtlich wird. Signifikant mit Blick auf die Funktion der Äußerung ist hierbei, dass mit der VS-Stellung eine neue, an den vorausgehenden Text nicht unmittelbar anknüpfbare Information eingeführt wird, bei der, wie die modifizierte Passage in (5b) zeigt, SV die Kohäsion gefährden würde. Berücksichtigt man die sich aus Auxiliar und Partizip ergebende Klammer, lässt sich die Hypothese aufstellen, dass es sich hierbei um den ikonischen Reflex einer 'globalen Sachverhaltsdarstellung' handelt, also ein ausdruckstrukturelles Mittel zur Profilierung eines kommunikativ thetischen Profils. In (5a) werden also im Sinne Sasses global Rahmenbedingungen präsentiert, jedoch nicht in unmittelbarer Nähe zum 'eingerahmten' Ereignis, sondern quasi in umgekehrter Reihenfolge: Bevor sich der Rezipient der invertierten Darstellung der Sachverhalte bewusst werden kann, wird im Text die Ursache für den Angriff auf die Stadt Ravenna antizipiert. Eine explizite Verbalisierung der Relation zwischen der fraglichen Äußerung und dem Ko-Text, etwa durch ein Adverb bzw. eine Konjunktion (etwa assaltare Ravenna, poiché... 'Ravenna angreifen, da...') unterbleibt. Hier zeigt sich ein wesentlicher Unterschied zu typischen thetischen Belegen, da in (5a) - im Vergleich zu (1a) - der Ko-Text eine thetische Lesart regelrecht erzwingt, um die Äußerung überhaupt anschlussfähig zu machen. Darüber hinaus wird hierbei auch nicht prospektiv la città di Ravenna 'die Stadt Ravenna' einfach als Referent und somit möglicher Gegenstand der folgenden Ausführungen etabliert, sondern es wird vielmehr der gesamte Sachverhalt der Herrschaft Ostasio da Polentas über Ravenna als Information eingeführt.

Auch in Beleg (6), allerdings aus etwas anderen Gründen, ist das Verhältnis von Ko-Text und Serialisierungsmuster als auffällig zu bezeichnen:
[...] i Saraceni tenevono la Sicilia e ogni dì scorrevono i liti di Italia; per la qual cosa Guglielmo convenne con il principe di Capua e di Salerno e con Melorco greco, che per lo imperadore di Grecia governava la Puglia e la Calavria, di [assaltare la Sicilia], e, seguendone la vittoria, si accordorono che qualunche di loro della preda e dello stato dovesse per la quarta parte participare. [Fu [la impresa $\left.\mathbf{a}_{\mathbf{i}}\right]$ felice $]_{\mathbf{j}}$; e cacciati i Saraceni, occuporono la Sicilia. Dopo [la quale vittoria $]_{\mathbf{j}}$, Melorco [...] prese la possessione dell' isola per lo imperadore, e solamente divise la preda. (Istorie fiorentine I, 16) '[...] die Sarazenen hielten Sizilien besetzt und unternahmen täglich Streifzüge längs der Küsten Italiens. Deshalb kam Wilhelm mit den Fürsten von Capua und Salerno und mit Melorkos, dem griechischen Statthalter in Apulien und Kalabrien, überein, [Sizilien anzugreifen $]_{i}$, so dass sie, im Falle des Sieges, beschlossen, dass jedem der vierte Teil des Landes und der Beute gehören sollte. [Es war [das Unternehmen ${ }_{i}$ ] erfolgreich] $]_{j}$ : sie verjagten die Sarazenen und besetzten die Insel; [nach diesem Sieg $]_{\mathbf{j}}$ nahm Melorkos [...] vom Lande Besitz für den Kaiser und teilte nur die Beute.'

In (6) liegt in $\mathrm{Fu}$ la impresa felice 'Es war das Unternehmen erfolgreich' zunächst einmal semantisch gesehen eine prädikative Struktur vor. Wie der definite Artikel anzeigt, geht es in der fraglichen Passage um eine zuvor genannte impresa 'Unternehmen', die einen erfolgreichen Ausgang hat. Die impresa 'Unternehmen' ist insoweit also themafähig. Isoliert betrachtet suggeriert der Aussageinhalt der Äußerung, dass es sich um einen 'Erzählerkommentar' zum Ausgang der impresa 'Unternehmen' handle (kategorische Äußerung) und die 'Aufgabe' der Verb-Subjekt-Serialisierung mithin darin bestünde, den Ebenenwechsel 
von der Erzählung zum Kommentar zu markieren. Eine genauere Analyse des Kontexts lässt allerdings schnell die Problematik einer solchen Interpretation deutlich werden.

Die erzählende Instanz legt in dem fraglichen Abschnitt zunächst die Pläne der drei Protagonisten, Guglielmos, des Prinzen von Capua und Salerno sowie Melorcos, dar, die planen, Sizilien anzugreifen und ,im Falle des Sieges“ - man beachte die konditionale Lesart des Gerunds - Land und Beute aufzuteilen. Im Anschluss wird mit Fu la impresa felice 'Es war das Unternehmen erfolgreich' der Erfolg des Unternehmens festgestellt und es werden die Konsequenzen daraus berichtet, nämlich die Vertreibung der Araber, die Besetzung Siziliens und schließlich Melorcos 'Vertragsbruch'.

Wie der Ko-Text also offenbart, fungiert $F u$ la impresa felice 'Es war das Unternehmen erfolgreich' gerade nicht als 'klassischer' Erzählerkommentar, sondern erscheint auf der Ebene der textuellen Sachverhaltsdarstellung als elementarer Bestandteil der Ereigniskette. Damit ist natürlich noch nicht die Frage beantwortet, warum VS anstelle von prinzipiell möglichem SV auftritt. Da der Subjektreferent impresa 'Unternehmen' themafähig ist, erhielte man bei SV quasi automatisch eine kategorische Lesart der Äußerung, wodurch auf der Ebene der Darstellung zum einen die Ereignishaftigkeit des Sachverhalts textpragmatisch deutlich reduziert würde. Zum anderen würde la impresa 'das Unternehmen' thematische Kontinuität signalisieren, ${ }^{11}$ sodass seguendone la vittoria, si accordorono... 'so dass sie, im Falle des Sieges, ...' zu einer reinen 'Parenthese' heruntergestuft würde, obwohl Melorcos Vertragsbruch (prese la possessione dell'isola... '[Melorkos] nahm vom Lande Besitz') Ziel der Darstellung ist. Und schließlich würde der Autor möglicherweise die Textkohäsion beeinträchtigen, weil zwischen der Planung des Angriffs und der Feststellung seines erfolgreichen Verlaufs der Angriff selbst nur implizit bliebe. VS blockiert also die kategorische Lesart der Äußerung.

Die Analyse des Beispiels zeigt wiederum, dass es sinnvoll ist, zwischen der syntaktischsemantisch gesehen prädikativen Struktur des Satzes und der textpragmatisch intendierten, hier thetischen, Sachverhaltsdarstellung zu unterscheiden. Beachtenswert ist hierbei allerdings die Doppelfunktion der VS-Struktur, die aus einer gewissen Interferenz zwischen der syntaktisch-semantischen und pragmatischen Ebene resultiert: Zum einen wird die tatsächliche Umsetzung des Plans in einer eventiven Perspektivierung und zum anderen gleichzeitig der Erfolg der Sache affirmiert. Wurde in (5a) die Pointierung eines zentralen Sachverhalts dadurch erzielt, dass der fragliche Sachverhalt unter Zuhilfenahme der Serialisierung VS antizipiert worden war, wird in (6) die besondere Hervorhebung des Sachverhalts gerade durch den ambivalenten pragmatischen Status der Äußerung bzw. der Konstituenten erzielt.

Ließ sich allerdings in (5a) das scheinbare Spannungsverhältnis zwischen dem propositionalen Gehalt der Äußerung und dem Ko-Text durch die Deutung im Sinne eines textpragmatisch thetischen Profils lösen und war in (6) eine (weitgehende) pragmatische 'Monosemierung' des vorliegenden kommunikativen Profils durch eine detaillierte Analyse des KoTexts möglich, scheint der Ko-Text in den Belegen (7) und (8) keine klaren Hinweise in Bezug auf das vorliegende kommunikative Profil zu liefern. Man ist dabei mit Darstellungsmustern befasst, die aufgrund ihrer Rekurrenz durchaus als spezifisch für die Istorie fiorentine bezeichnet werden können.

(7) a. Mandò [Pietro Fregoso, doge della Repubblica di Genova] per tanto oratori a Carlo VII re di Francia, e gli offerì lo imperio di Genova. Accettò Carlo la offerta, e a prendere la possessione di quella città vi mandò Giovanni d'Angiò [...]. (Istorie fiorentine VI, 35)

11 Streng genommen wäre noch zu prüfen, ob zwischen der Hypothese eines Siegs (seguendone la vittoria! 'im Falle des Sieges') und dem tatsächlichen Erfolg eine anaphorische Beziehung gesehen werden kann. 
'Darum sandte er [Pietro Fregoso, Doge der Republik Genua] Abgeordnete nach Frankreich an Karl VII. und bot ihm die Herrschaft über Genua an. Karl nahm das Angebot an und sandte zur Besitznahme Johann von Anjou [...].'

b. Fu data per tanto la commissione a messer Rinaldo che fusse con Giovanni, e vedesse di tirarlo nella sentenza loro. [Cap. 10] Esequì il Cavaliere la commissione, e con tutti quelli termini seppe migliori lo confortò a pigliare questa impresa con loro, e non volere, per favorire una moltitudine, farla audace con rovina dello stato e della città. (Istorie fiorentine IV, 9f)

'So wurde denn Messer Rinaldo der Auftrag erteilt, zu Giovanni zu gehen und zu versuchen, ihn zu ihrer Partei herüberzuziehen. [Kap. 10] Der Cavaliere führte den Auftrag aus und bot alle seine Gründe und die besten Worte auf, ihn zu veranlassen, ihrem Plane seine Zustimmung zu geben, statt, durch Begünstigung der Menge, diese zum Nachteil des Staates und der Stadt aufzuregen.'

(8) a. E mandorono di nuovo a papa Bonifazio perché pensasse al rimedio, se non voleva che quella città, che era stata sempre scudo della Chiesa, o rovinasse o diventasse ghibellina. Mandò pertanto il Papa in Firenze Matteo d'Acquasparta, cardinale Portuese, legato; e perché trovò difficultà nella parte bianca, la quale per parergli essere più potente temeva meno, si partì di Firenze sdegnato, e la interdisse; di modo che la rimase in maggiore confusione che la non era avanti la venuta sua. (Istorie fiorentine II, 17)

'Darum sandten sie zu Papst Bonifaz, damit er auf eine Abhilfe denke, wenn er nicht wolle, dass die Stadt, welche stets der Kirche Schild gewesen, zugrunde gehe oder gibellinisch werde. Der Papst sandte den Kardinal von Porto, Matteo von Acquasparta, als Legat nach Florenz, und da dieser bei den Weißen auf Schwierigkeiten stieß, indem dieselben weniger fürchteten, weil sie sich für die Stärksten hielten, so zog er unwillig aus Florenz ab und ließ die Stadt im Interdikt. So herrschte dort nach seiner Abreise größere Verwirrung als vor seiner Ankunft.'

b. E per ciò ricorsono al Pontefice, pregandolo che a questi umori mossi quello rimedio che per loro non vi potevono porre con la sua autorità vi ponesse. Mandò il Papa per messer Veri, e lo gravò a fare pace con i Donati [...]. (Istorie fiorentine II, 17)

'Deshalb wandten sie sich an den Papst und baten ihn, dass er in seiner Machtvollkommenheit ein Mittel gegen diesen Hader anwende, welches sie mit eigener Macht nicht anwenden könnten. Der Papst schickte nach Messer Vieri und gab ihm auf, mit den Donati Frieden zu schließen.’

Gemein ist den Beispielen, dass in zwei aufeinanderfolgenden Sätzen jeweils quasi komplementäre Sachverhalte berichtet werden: In (7a) wird im ersten Satz ein Angebot unterbreitet gli offeri lo imperio di Genova '[Karl VII.] bot ihm die Herrschaft über Genua an', das im zweiten Satz angenommen wird - accettò Carlo la offerta 'Karl nahm das Angebot an' in (7b) wird die Erteilung eines Auftrags - Fu data per tanto la commissione 'So wurde denn der Auftrag erteilt' - und seine Ausführung - Esequi il Cavaliere la commissione 'Der Cavaliere führte den Auftrag aus' - dargelegt.

Trifft im Grunde Ähnliches auf die Belege in (8) zu, so unterscheiden sich diese von jenen in (7) dadurch, dass sie inhaltlich wie lexikalisch etwas detailreicher ausgeführt sind. In (8a) und $(8 \mathrm{~b})$ wird jeweils eine Bitte an den Papst referiert, die genaue Formulierung bezieht sich jedoch nicht nur auf den reinen Sprechakt 'Bitte', sondern beinhaltet dann auch die entsprechenden Argumente, die die Entscheidungsfindung des Papstes im Sinne der Bittsteller motivieren sollen. Folglich würde man mehr als in (7a) und (7b) erwarten, auch etwas über den Prozess der Entscheidungsfindung seitens des Papstes zu erfahren. Wie jedoch Mandò 
pertanto il Papa in Firenze Matteo d'Acquasparta 'Der Papst sandte den Kardinal von Porto, Matteo von Acquasparta, als Legat nach Florenz' und Mandò il Papa per messer Veri 'Der Papst schickte nach Messer Vieri' offenbaren, bleibt auch hier, wie in (7a) und (7b), dieser Schritt aus, sodass die textuelle Sachverhaltsdarstellung die unverzügliche Sukzession der Handlungsschritte suggeriert.

Unabhängig von diesen Unterschieden zwischen (7) und (8) kann zunächst festgehalten werden, dass die jeweiligen postverbalen Subjektreferenten gegeben, also themafähig, sind. Hinsichtlich der Frage einer kategorischen oder thetischen Lesart der Äußerungen liefert (zumindest) der underlying question-Test keine klaren Ergebnisse, denn die Äußerungen können sowohl auf Che fece $x$ poi? 'Was hat X dann getan' als auch auf Che successe poi? 'Was geschah dann?' antworten. In letzterem Fall kann die VS-Struktur entweder der Blockierung einer kategorischen Interpretation der Äußerung oder eben der Signalisierung einer ereignishaft-thetischen Lesart dienen. Da in (7) und (8) syntaktisch gesehen auch SV denkbar wäre, was klar zu einer kategorischen Lesart führen würde, ist nach dem kommunikativen Mehrwert einer kategorisch interpretierten VS-Serialisierung $\mathrm{zu}$ fragen. Unabhängig von kategorischem SV oder VS ist in beiden Fällen das Denotat des Verbs qua Auswahl aus einer Option als rhematisch einzustufen, schließlich kann man ein Angebot etc. auch ablehnen. Durch die Position des Verbs in der äußerungsinitialen, kommunikativ prominenteren Position wird die Tatsache der positiven Entscheidung noch stärker hervorgehoben, sodass man von einer Weiterrhematisierung im Sinne von Oesterreicher (1991) sprechen kann, in deren Windschatten das Subjekt dann in die postverbale Position rückt.

Vor dem Hintergrund der Überlegung, dass VS hier den Zweck der Weiterrhematisierung des Denotats des Verbs erfüllt, ist allerdings auch nochmals auf die Unterschiede zwischen den Belegen in (7) und (8) einzugehen. Während hinsichtlich der Zuordnung zum thetischen oder kategorischen Profil im Fall von (7) die Vermutung einer intentionalen Ambiguität nicht von der Hand zu weisen ist, da hier die pragmatische Interpretation zwischen thetisch und kategorisch zu oszillieren scheint, ${ }^{12}$ spricht bei (8) eine kontextuelle Besonderheit eher für eine kategorische Interpretation: Innerhalb der vorangestellten 'Argumente' (se non voleva... 'wenn er nicht wolle' und che ... ponesse 'dass er ... anwende') ist der Papst bereits als Subjekt kodiert, sodass die referentielle Kontinuität durch gleichbleibende syntaktische Kodierung sekundiert wird und eine Art thematische Kontinuität entsteht. Eine abschließende Einschätzung der in (7) und (8) vorliegenden pragmatischen Verhältnisse wird gleichwohl erst nach einer systematischen Analyse solcher Konstellationen möglich sein.

Eine wesentlich präzisere Analyse verlangen zwar vergleichbare Belege, in denen allerdings zusätzlich adverbiale Elemente auftreten:
Di che ne seguì che i Vandali, i quali avevano occupata quella parte della Spagna detta Betica, sendo combattuti forte da i Visigoti, e non avendo rimedio, furono da Bonifazio, il quale per lo Imperio governava Affrica, chiamati che venissero ad occupare quella provincia; perché, sendosi ribellato, temeva che il suo errore non fusse dallo Imperadore ricognosciuto. Presono i Vandali, per le cagioni dette, volentieri quella impresa, e sotto Genserico loro re, si insignorirono d'Affrica. (Istorie fiorentine I, 1)
'Da nun die Vandalen, die sich in jenem Teile Spaniens, den man Baetica nennt, niedergelassen hatten, von den Westgoten hart gedrängt wurden und keinen Ausweg wussten, so geschah es, dass sie von Bonifacius, kaiserlicher Statthalter in Afrika,

12 Dass in Beispiel (7b) im Rahmen der erst im 19. Jahrhundert eingefügten Kapiteleinteilung der 'Schnitt' an genau dieser Stelle gemacht worden ist, lässt vermuten, dass Esequì il Cavaliere... 'Der Cavaliere führte ...' als thetische Äußerung gelesen wurde. 
gerufen wurden, um diese Provinz zu besetzen. Weil dieser sich empört hatte, fürchtete er, der Kaiser werde die von ihm begangenen Fehler erkennen. Die Vandalen ließen sich gerne aus den genannten Gründen auf das Unternehmen ein und bemächtigten sich Afrikas unter ihrem Könige Genserich [...].'

Analog zu (7) und (8) geht es hier um die Bitte Papst Bonifatius' an die Vandalen, ihn bei der Besetzung der Provinz Afrika zu unterstützen, worauf diese auch eingehen. Die Vandali 'Vandalen' sind klar vorerwähnt und mithin themafähig; ferner wird, ähnlich wie in (8), die Darstellung der Ereignisse (furono chiamati 'wurden gerufen' - presono 'ließen sich ein') durch eine kurze Erläuterung der Beweggründe des Papstes (perché, sendosi ribellato... 'weil dieser sich empört hatte...') unterbrochen.

Durch die ko-textuelle Ähnlichkeit zu den Belegen (7) und (8) scheint in diesem Punkt die Problematik in Bezug auf die Zuordnung zu einer kategorischen oder thetischen Lesart identisch, da auch hier der underlying question-Test zu keinem klaren Ergebnis führt: Eine thetische Lesart würde implizieren, dass das tatsächliche Eingehen auf die Bitte seitens der Vandali 'Vandalen' lediglich als ein für den Fortgang der Ereignisse nötiges Element referiert werden soll. Eine kategorische Lesart hingegen hätte zur Folge, dass einerseits den Vandali textpragmatisch ein deutlicherer Stellenwert als Handlungsträger zugewiesen werden würde, andererseits aber, analog zu (7) und (8), das Denotat des Verbs gleichwohl als die kommunikativ relevantere Information ausgewiesen würde.

Im Vergleich zu (7) und (8) sind allerdings in (9) per le cagioni dette 'aus den genannten Gründen' und volentieri 'gerne' bei Bestimmung des kommunikativen Profils der Äußerung zu berücksichtigen. Man könnte zunächst annehmen, mit volentieri 'gerne' fließe lediglich ein Erzählerkommentar ein, ${ }^{13}$ tatsächlich aber mahnt die vorgängige Explizierung per le cagioni dette 'aus den genannten Gründen', die zu Beginn des Belegs erwähnte Ausgangssituation mit in Betracht zu ziehen: sendo combattuti forte da $i$ Visigoti, e non avendo rimedio 'da sie von den Westgoten hart gedrängt wurden und keinen Ausweg wussten'. Dem Rezipienten soll also ausdrücklich die Ausgangssituation der Vandali 'Vandalen' in Erinnerung gerufen und insoweit vermittelt werden, dass die Vandali 'Vandalen' der Bitte des Papstes letztlich nur aus eigennützigen Motiven entsprechen. Der anaphorische Verweis mit per le cagioni dette 'aus den genannten Gründen' macht also die Subjektreferenten zu Entitäten, über die pragmatisch neue Information gegeben wird.

Wenngleich Konstellationen wie die hier vorgestellten noch auf mögliche Vorbilder in der lateinischen Historiographie hin zu überprüfen sind, zeigt sich, dass Machiavelli mit funktional lang etablierten Ausdrucksmustern (thetisches/kategorisches VS) ein Komplexitätsniveau der sprachlichen Sachverhaltsdarstellung erreicht, das bis dahin in der volkssprachlichen Historiographie kaum anzutreffen sein dürfte.

\section{Fazit und offene Fragen}

Bereits eingangs ist darauf hingewiesen worden, dass das Ziel dieses Beitrags weniger darin bestand, Fragen zu beantworten als vielmehr eine Grundlage zu schaffen, um neue Fragen mit Blick auf syntaktisch-textorganisatorische Aspekte im Italienischen des Cinquecento formulieren zu können. Zentrales Anliegen war es in diesem Zusammenhang, zu zeigen, dass in den Istorie fiorentine Machiavellis die VS-Serialisierung in ihren unterschiedlichen Funktionen

13 Dass es sich nicht um einen reinen Erzählerkommentar handeln kann, zeigt der Umstand, dass Presono... quella impresa 'ließen sich ... auf das Unternehmen ein' Bestandteil der Ereigniskette ist. 
(vgl. Kap. 3.1) bewusst als Technik der Textstrukturierung Anwendung findet (vgl. Kap. 3.2 und 3.3); dabei war auch die alternative SV-Linearisierung in die Betrachtung einzubeziehen. Das Spiel mit diesen Serialisierungsmustern erwies sich dabei als ein textpragmatisches Verfahren, mit dem das kommunikative Profil von Äußerungen bzw. die pragmatische Funktion bestimmter Konstituenten der jeweiligen Textstrukturierungsintention angepasst werden kann.

Außer Zweifel steht dabei, dass Machiavelli bereits seit langer Zeit in der Volkssprache dokumentierte syntaktische Muster nicht einfach imitiert, sondern kontext-sensitiv einsetzt bzw. an seine Zielsetzungen adaptiert und sie so fortschreibt. Wie v.a. die Beispiele (7) und (8) vermuten lassen, ist hierbei freilich auch die Herausbildung gewisser idiosynkratischer Strukturen nicht auszuschließen.

Für eine weitergehende und systematischere Auswertung der Belege dürfte es erforderlich sein, diskurstraditionelle Faktoren stärker und genuin sprachliche Faktoren noch detaillierter zu berücksichtigen. Im Bereich der Ersteren ist zu denken an den Umgang mit literarischen Modellen, an Einflüsse verschiedener Diskurstraditionen sowie an die Rolle rhetorischer Techniken; im zweiten Bereich wären Faktoren wie eine zunehmend komplex gewordene Periodenstruktur, das Maß der Ausschöpfung syntaktischen Variationspotentials und Formen des Ausdrucks von Kohärenz- und Kohäsionsbeziehungen in Betracht zu ziehen. Darüber hinaus wäre auch in methodischer Hinsicht zu fragen, inwieweit Beschreibungsbegriffe, die auf der Grundlage gegenwartssprachlicher Texte entwickelt worden sind, sich auf Texte älterer Sprachstufen anwenden lassen.

\section{Literatur}

Blumenthal, P. (1980): Die Stilistik der Subjektinversion im Italienischen. Italienische Studien 3. 119-131.

Blumenthal, P. (1990): Textorganisation im Französischen vom Mittelalter zur Klassik. Zeitschrift für französische Sprache und Literatur 100. 25-60.

Blumenthal, P. (2000): Textlinguistik und Geschichtswissenschaft. In: Brinker, K., Antos, G., Heinemann, W. \& Sager, S. (Hg.), Text- und Gesprächslinguistik. Ein internationales Handbuch zeitgenössischer Forschung. 1. Halbband. Berlin, New York: De Gruyter. 797-803.

Cernecca, D. (1982): L'inversione del soggetto nella prosa del Principe di N. Machiavelli. In: Branca, V. et al. (Hg.), Il Rinascimento. Aspetti e problemi attuali. Atti del X Congresso dell'Associazione internazionale per gli studi di lingua e letteratura italiana. Belgrado, 17-21 aprile 1979. Firenze: Olschki. 259-273.

Chafe, W. L. (1976): Givenness, Contrastiveness, Definiteness, Subjects, Topics, and Point of View. In: Li, C. N. (Hg.), Subject and Topic. New York, San Francisco, London: Academic Press. 25-55.

Colussi, D. (2014): Cronaca e storia. In: Antonelli, G., Motolese, M. \& Tomasin, L. (Hg.), Storia dell'italiano scritto. II. Prosa letteraria. Roma: Carocci. 119-152.

Compagni, Dino (1914): Chronik des Dino Compagni. Von den Dingen, die zu seiner Zeit geschehen sind. Übersetzt und eingeleitet von Ida Schwarz. Jena: Eugen Diederichs.

Daneš, F. (1964): A three-level approach to syntax. Travaux linguistiques de Prague 1. 225-240.

Ferranti, L. (2010): Aspetti della sintassi e della testualtità della storiografia toscana cinquecentesca. Roma: Università degli Studi Roma Tre, Dipartimento di Italianistica (Dissertation).

Fesenmeier, L. (2003): L'ordine dei costituenti in toscano antico. Padova: Unipress.

Fesenmeier, L. (2004): Inversione del soggetto e strutturazione del testo nell'italiano antico. In: Zaccarello, M. \& Tomasin, L. (Hg.), Storia della lingua e filologia. Per Alfredo Stussi nel suo sessantacinquesimo compleanno. Firenze: Edizioni del Galluzzo. 101-120.

Franceschini, F. (2011): Machiavelli, Niccolò. In: Simone, Raffaele (Hg.), Enciclopedia dell'italiano. Roma: Istituto della Enciclopedia Italiana. 845-847.

Giovanardi, C. (2004): Aspetti della sintassi e della testualità nelle Istorie fiorentine di Niccolò Machiavelli. In: D’Achille, P. (Hg.), Generi, architetture e forme testuali. Atti del VII convegno SILFI (Roma $1^{\circ}-5$ ottobre 2002). Bd. 2. Firenze: Cesati. 601-619.

Harth, D. (2007): Geschichtsschreibung. In: Ueding, G. (Hg.), Historisches Wörterbuch der Rhetorik. Bd. 3. Tübingen: Niemeyer. 832-870. 
Hatcher, A. G. (1956): Syntax and the Sentence. Word 12. 234-250.

Kersten, L. (2015): L'arte della brevità - Textorganisation und Satzgliedstellung in Machiavellis Istorie fiorentine. Erlangen: FAU Erlangen-Nürnberg, Institut für Romanistik (Zulassungsarbeit).

Lambrecht, K. (1994): Information structure and sentence form. Cambridge, New York, Melbourne: Cambridge University Press.

LIZ: Stoppelli, P. \& Picchi, E. (Hg.) (2001): Letteratura Italiana Zanichelli 4.0. Bologna: Zanichelli.

Machiavelli, N. (1934): Geschichte von Florenz. Dt. von Alfred von Reumont. Hg. von Ludwig Goldschneider. Wien: Phaidon.

Machiavelli, N. (2010): Istorie fiorentine. In: Machiavelli, N., Opere storiche. A cura di Alessandro Montevecchi e Carlo Varotti. Roma: Salerno Editrice. 77-785.

Matucci, A. (1991): Machiavelli nella storiografia fiorentina. Per la storia di un genere letterario. Firenze: Olschki.

Neumann-Holzschuh, I. (1997): Die Satzgliedstellung im Spanischen. Tübingen: Niemeyer.

Oesterreicher, W. (1991): Valenz und Informationsstruktur. In: Koch, P. \& Krefeld, T. (Hg.), Connexiones Romanicae. Dependenz und Valenz in romanischen Sprachen. Tübingen: Niemeyer. 267-292.

Oesterreicher, W. (1993): Verschriftung und Verschriftlichung im Kontext medialer und konzeptioneller Schriftlichkeit. In: Schaefer, U. (Hg.), Schriftlichkeit im frühen Mittelalter. Tübingen: Narr. 267-292.

Reinhart, T. (1981): Pragmatics and Linguistics: An Analysis of Sentence Topics. Philosophica 27. 53-94.

Renzi, L. (2008): Storia interna dell'italiano: morfosintassi e sintassi. In: Ernst, G., Gleßgen, M.-D. \& Schmitt, C. \& Schweickard, W. (Hg.), Romanische Sprachgeschichte. Ein internationales Handbuch zur Geschichte der romanischen Sprachen. Bd. 3. Berlin, New York: De Gruyter. 2830-2847.

Rigon, A. (2007): La sintassi del periodo nelle 'Istorie fiorentine' di Machiavelli e nella 'Storia d'Italia' di Guicciardini. Stilistica e metrica 7. 77-129.

Sasse, H.-J. (1987): The thetic/categorical distinction revisited. Linguistics 25. 511-580.

Sasse, H.-J. (1995): 'Theticity' and VS order: a case study. In: Matras, Y. \& Sasse, H.-J. (Hg.), Verb-Subject Order and Theticity in European Languages. Berlin: Akademie Verlag. 3-31.

Sasse, H.-J. (1996): Theticity. Köln: Institut für Sprachwissenschaft der Universität zu Köln.

Scavuzzo, C. (2003): Machiavelli. Roma: Carocci.

Schrott, A. (2015): Nähe und Lebendigkeit in privaten Briefen aus diskurstraditioneller Sicht. In: Bernsen, M., Eggert, E. \& Schrott, A. (Hg.), Historische Sprachwissenschaft als philologische Kulturwissenschaft. Bonn: Bonn University Press, Vandenhoeck \& Ruprecht. 479-497.

Selig, M. (1992): Die Entwicklung der Nominaldeterminanten im Spätlatein. Tübingen: Narr.

Stark, E. (1997): Voranstellungsstrukturen und "topic”-Markierung im Französischen. Tübingen: Narr.

Stefinlongo, A. (2004): Proforme relative e coesione testuale nelle Istorie fiorentine di Niccolò Machiavelli. In: D'Achille, P. (Hg.), Generi, architetture e forme testuali. Atti del VII convegno SILFI (Roma $1^{\circ}-5$ ottobre 2002). Bd. 2. Firenze: Cesati. 621-632.

Strawson, P. F. (1964): Identifying reference and truth-value. Theoria 30. 96-118.

TLIO: Corpus Tesoro della Lingua Italiana delle Origini. Stand: 3.12.2015. URL: http://tlioweb.ovi.cnr.it. Zugriff am: 04.01.2017.

Ulrich, M. (1985): Thetisch und Kategorisch: Funktion der Anordnung von Satzkonstituenten am Beispiel des Rumänischen und anderer Sprachen. Tübingen: Narr.

Venier, F. (2002): La presentatività. Sulle tracce di una nozione. Alessandria: Edizioni dell'Orso. 


\title{
Zu germ. $* \bar{e}^{l}$ im Präteritum der germanischen starken Verben
}

\author{
Patrick Mächler (Zürich)
}

\begin{abstract}
This paper addresses one of the most disputed issues in Proto-Germanic verb morphology, the rise of $* \bar{e}^{-1}$ as a co-marker of preterite plural forms in classes IV and V of strong verbs, as seen, for example, in *ber-a- 'to carry' - *berr-um 'we carried' or *geb-a- 'to give' - *gēb-um 'we gave'. Unlike other tense-marking ablaut grades of Proto-Germanic strong verbs, $*^{-} \bar{e}^{l}$ cannot be traced back to Indo-European ablaut. In this paper, I put under scrutiny three different accounts of its origin: the "aorist hypothesis", according to which the lengthened grade can be linked to IndoEuropean aorist formations; Schumacher's (2005) "bigêtun-rule" that yields lautgesetzlich ${ }^{-} \bar{e}^{-}$in *TeT-roots; and the so-called "analogy hypothesis", which assumes analogical spread of $* e^{-1}$ from contexts where it developed in accordance with well-established sound laws. I will argue that only the latter account entails no unwarranted assumptions, whereas the first two would force us to posit ad hoc morphological or phonological developments. In the second part of the paper, I debunk the claim that $* e^{I}$ had too small a basis to spread (viz. only one verb or only a couple of verbs). Whether or not a morphological pattern becomes productive depends on more factors than type frequency alone, although many morphological theories and models would like to make us believe otherwise. While acknowledging the insecurity that accompanies morphological reconstruction, it will be argued that by taking into account two central ideas of Natural Morphology - linking inflection-class membership to extramorphological features such as a lexeme's phonological make-up, and system-dependent normalcy of markers - the analogical spread of $* \bar{e}^{-}$will turn out to be nothing more extraordinary than, say, the rise of Umlaut as a plural marker in German noun morphology.
\end{abstract}

\section{Einleitung ${ }^{1}$}

Dieser Beitrag behandelt Ursprünge und Ausbreitung des Vokals $* \bar{e}^{-1}$ im Prät.Pl. der Klassen IV und V der germanischen starken Verben, wie ihn beispielsweise ahd. gāabun 'sie gaben' zu gëban oder got. nếmun 'sie nahmen' zu niman zeigen (ahd. $\bar{a}$, got. $\hat{e}<$ germ. ${ }^{*} \bar{e}^{-1}$ ). ${ }^{2,3}$ Dieser Präteritalvokal hat, anders als die Stammvokale im Prät.Sg. der Klassen I-V und im Prät.Pl.

1 Dieser Artikel ist im Zuge der Bearbeitung eines vom Schweizerischen Nationalfonds unterstützten Dissertationsprojektes an der Universität Zürich entstanden (Projektnummer 155501).

2 Entsprechend dem indo- und altgermanistischen Usus bezeichnet ein Asterisk vor einem Laut oder einer Wortform, dass es sich um ein Rekonstrukt handelt. Ein der Wortform nachgestellter Asterisk gibt an, dass das Lexem zwar belegt ist, die Nennform aber zufälligerweise im Korpus fehlt. Hypothetische, zu Illustrationszwecken angesetzte Formen werden mit $\dagger$ gekennzeichnet. Das Zeichen ,,“" steht für die streng lautgesetzliche Entwicklung einer Form, wohingegen,$\rightarrow$ “ für die Beteiligung analogischer Prozesse steht. Als Coversymbole finden in diesem Beitrag Verwendung: $K=$ irgendein Konsonant; $K_{0}=$ Konsonanten in beliebiger Anzahl, inkl. 0; $V=$ irgendein Vokal; $R=$ Resonant; $R=$ silbischer Resonant; $N=$ Nasal; $T=$ Obstruent; $H$ = Laryngal unbekannter Qualität.

3 Die Indexierung dient der Unterscheidung von einem geschlosseneren langen $e$-Laut, ${ }^{*} \bar{e}^{2}$. Zum Lautwert von germ. ${ }^{2} \bar{e}^{2}$ vgl. zuletzt Kroonen (2013: xxiiif.). 
der Klassen I-III, keine erkennbare indogermanische Entsprechung. Seine Anfänge reichen deswegen in die nachgrundsprachliche, vorgerm. oder germ. Zeit zurück. Es erstaunt daher nicht, dass die Herleitung des Markers des Prät.Pl. (und, weitaus weniger, dessen Ausbreitung) ein seit den Anfängen der modernen historischen Sprachwissenschaft im 19. Jh. vielbeachtetes Problem darstellt. Dieser Beitrag verfolgt nicht das Ziel, einen völlig neuen Ansatz $\mathrm{zu}$ präsentieren. Im Gegenteil werde ich mich - mit einigen Anpassungen - im Grundsatz einer bereits mehrfach vorgeschlagenen Herleitung anschließen, die mit einer analogischen Ausbreitung ausgehend von einem oder einigen wenigen Verben mit lautgesetzlichem $* \bar{e}^{-1}$ rechnet (im Folgenden „Analogiehypothese“ genannt). Die Ursachen für diese Ausbreitung sind aber, wie zu zeigen sein wird, woanders zu suchen, als das die Forschung bis anhin getan hat. Das hier vertretene Szenario berührt dabei insbesondere die Frage, was die Produktivwerdung eines flexivischen Musters begünstigt. Aus diesem Grund behandle ich dieses auf den ersten Blick rein altgermanistische Problem vor dem Hintergrund allgemeintheoretischer Betrachtungen zur Repräsentation morphologisch komplexer Wörter und der Produktivität in Theorien und Modellen wie der Natürlichen Morphologie, dem Bybee'schen Netzwerkmodell, dem Duale-Routen-Modell oder dem dual-route-race-Modell.

Im folgenden Kapitel wird das System der germ. starken Verben in seinen grundsprachlichen Bezügen ausschnittsweise und übersichtsartig dargestellt; ferner werden die am germ. starken Verb markierten Kategorien und ihre Markertypen betrachtet. Kapitel 3 geht der Frage nach dem Ursprung von germ. ${ }^{*} \bar{e}^{-1}$ im System der starken Verben nach, wobei drei Erklärungsansätze im Detail erörtert und deren Plausibilität diskutiert werden: Die Aoristhypothese, die eine Einwirkung grund- oder nachgrundsprachlicher dehnstufiger Aoriste auf die Entwicklung des germ. Prät. annimmt, die sog. „bigêtun-Regel“, die den Langvokal als lautgesetzlichen Fortsetzer von Obstruentenclustern (Schwund mit Ersatzdehnung) zu erklären versucht, sowie die bereits erwähnte Analogiehypothese. Ein besonderes Augenmerk liegt dabei auf den Argumenten, die für die Plausibilität der Analogiehypothese sprechen. Die Auseinandersetzung mit den Argumenten für die Analogiehypothese in Kapitel 4 offenbart Diskrepanzen hinsichtlich der Auffassung, was die Produktivität eines Flexionsmusters beeinflusst: Altgermanistische und theoretische Beiträge gehen von teils diametralen Prämissen aus. Kapitel 5 versucht, das Szenario einer analogischen Ausbreitung von germ. ${ }^{*} \bar{e}^{-1}$ im System der starken Verben im Lichte jüngerer Theorien, insbesondere unter Bezug auf Konzepte der Natürlichen Morphologie, aufzuzeichnen.

\section{Das germanische starke Präteritum und der Sonderfall der Klassen IV und V}

Anders als das wohl noch von Aspekt-Oppositionen geprägte und formenreiche idg. Verbalsystem zeigt das Spätgemeingerm. ein schlichtes binäres Tempussystem, in dem formal zwischen Präteritum und Präsens unterschieden wird. Für die Präteritalmarkierung stehen hauptsächlich zwei Ausdrucksverfahren zur Verfügung: Während in den sog. schwachen Verben ein Endungssatz germ. ${ }^{*}-\bar{\tau}^{n},{ }^{*}-\varangle \bar{e} z, \ldots$ das Präteritum markiert (nhd. machen - machte), ${ }^{4}$ übernehmen dies in den starken Verben in erster Linie Vokalalternationen im Stamm (nhd. singen - sang). Bei diesem stammmodulativen Verfahren handelt es sich um die Systematisierung indogermanischer Ablautalternationen, und zwar von denjenigen des grundsprachlichen Perfekts. Perfektstämme konnten im Idg. nur zu vergleichsweise wenigen Wurzeln ge-

4 Dabei handelt es sich um ein Suffix, das im Germ. aus einer Periphrase mit dem TUN-Wort grammatikalisiert wurde. Vgl. zur Genese und der Rekonstruktion des germ. Endungssatzes zuletzt Ringe (2006: 166-168), Hill (2010) und Mottausch (2013: 28-35). 
bildet werden, weil das Perfekt aspektuell relativ spezifisch war: In der Regel bezeichneten „Perfekta altindogermanischen Typs [...] einen Zustand des Handlungsträgers, der aus einer Handlung (meist Zustandsänderung) desselben Handlungsträgers in der Vergangenheit resultiert" [Hervorhebung im Original] (Tichy 2009: 93). Die grundsprachlichen Bildungsregeln des Perfekts sind dank des relativ einheitlichen Befunds der Tochtersprachen in der Indogermanistik wenig umstritten; ${ }^{5}$ als Charakteristika gelten

1) die der Wurzel vorgeschlagene Reduplikationssilbe (bestehend aus Wurzelanlaut und dem invariablen Reduplikationsvokal idg. *e),

2) Abtönstufe idg. *o im Singular resp. Schwundstufe $\neg$ im Plural sowie

3) für das Perfekt charakteristische Endungen.

Veranschaulichen lässt sich dies anhand der Wurzel idg. * $\hat{g} e u s-{ }^{-}$'kosten', ${ }^{6}$ deren Perf. in der 1.Sg. * $\hat{g} e$ - $\hat{g} o ́ u s-h_{2} e$, in der 1.Pl. * $\hat{g} e$-ĝus-mé lautet, woraus lautgesetzlich germ. * $(k e-) k a u s^{(a)}$, * (ke-)kuz-um ${ }^{(e)}$ (mit geneuerter Endung) und ahd. kōs, kurum 'wählte(n), prüfte(n)' resultierten. Das System der germ. starken Verben fußt daher weitgehend auf der idg. Alternation *e (im germ. Infinitiv und Präsens, sog. Averbo 1) $-{ }^{*} o$ (Prät.Sg., sog. Averbo 2) $-\neg$ (Prät.Pl., sog. Averbo 3). Üblicherweise wird in Grammatiken altgermanischer Sprachen auch noch ein viertes Averbo, das Partizip Präteriti, angegeben, das in gemeingerm. Zeit ausschließlich verbaladjektivische Funktion hatte und daher für unsere Fragestellung von peripherem Interesse ist.

Die Alternation idg. ${ }^{*} e-{ }^{*} o-\neg$ erscheint je nach lautlicher Umgebung im Germ. infolge von Vokalzusammenfall, Monophthongierung und Vokalepenthesen in $K R K$-Clustern stark umgebildet, vgl. Tabelle 1. Nach Ausweis der altgermanischen Korpussprachen wurde die Reduplikation noch in gemeingerm. Zeit in allen starken Verben mit Wurzelvokal germ. ${ }^{*} e$ $(=\mathrm{Kl}$. I-V) sowie bei solchen mit Wurzelreim *aK (K1. VI, vgl. *ak-a-, Präteritalstamm im Sg. und Pl. *ōk-) aufgegeben. Am Ende der gemeingerm. Zeit ist ihr Auftreten auf ca. 70 Verben beschränkt, die trotz sehr variabler Wurzelgestalt meist zu einer Klasse, der sog. Klasse VII, zusammengefasst werden, vgl. got. laí-lôt ' 'ließ' zu lêtan*, hai-hait 'hieß' zu haitan. Die Tilgung der Reduplikationssilbe wird meist als Abbau multipler Exponenz gewertet; der abtön- resp. schwundstufige Vokalismus habe die Formen gegenüber dem normalstufigen Präsens bereits ausreichend als Präterita markiert, vgl. u.a. Meid (1971: 53). Der Umstand, dass die Reduplikationssilbe unbetont war (also *ke-káus ${ }^{a},{ }^{*} k e-k u ́ z u m^{e}$ ?), hat die Tilgung zweifelsohne erleichtert. ${ }^{8}$ Einige Forscher wie Sverdrup (1927: 301) und Mottausch (2013: 18) nehmen an, dass die Dereduplikation durch Aoristeinfluss begünstigt worden sei. Demnach existierten der reduplikationslose Aorist und das reduplizierte Perfekt noch längere Zeit nebeneinander und entwickelten sich im Vorgerm. und Germ. zu Vergangenheitstempora im eigentlichen Sinne. Dabei war beiden ein schwundstufiger Pluralstamm eigen, vgl. den Aorist idg. * $g^{u} e ́ m-/ g^{u} m$ - $^{\prime}$ und das Perfekt * $g^{u} e-g^{u} o ́ m-/-g^{u} m m^{\prime}$. Mit einem allmählichen funktio-

Keine Einhelligkeit herrscht dagegen hinsichtlich der einzigen Ausnahme, dem reduplikationslosen Perfekt zur Wurzel idg. *ueid- 'erblicken', idg. *uóid-/uid- (vgl. 1.Sg. gr. oĩ $\delta \alpha$, awn. veit, nhd. weiß). Für unsere Diskussion ist diese Problematik allerdings vernachlässigbar. Dass das germ. starke Verb ursprünglich reduplizierte, beweisen vokalisch anlautende Verben von Kl. VII, die die Reduplikation einst lautgesetzlich verloren, sie zwecks Präteritalmarkierung aber wieder eingeführt haben müssen, vgl. got. ana-aí-auk 'fügte hinzu' zum Inf. ana-aukan (s. dazu Schumacher 2005: 595f.).

6 Ansatz der Wurzel nach Rix \& Kümmel (2001: 166).

7 Die Gotistik bedient sich der unterschiedlichen Digraphe <aí > und <ái >, um die etymologisch unterschiedliche Herkunft (und meist auch unterschiedliche Länge) dieser Laute zu markieren; <aí > (oft [ع]) geht meist

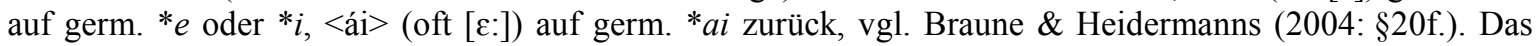
Diakritikon signalisiert also nicht den Wortakzent!

8 Der Akzent in starken Präterita kann bis mind. zum Ende der gemeingermanischen Zeit nicht auf der Reduplikationssilbe gelegen haben, da ein betonter Reduplikationssilbenvokal germ. *e im Got. als $i$ erschienen wäre, vgl. got. saí-so 'säte' (<ai > = [ع]), nicht †si-so, vgl. Voyles (1980: 94f.) und Hill (2009: 79-82). 
nalen Zusammenfall sei ein formaler einhergegangen, bei dem der reduplikationslose Aorist auf das sich herausbildende germanische Präteritum eingewirkt habe. Inwiefern die Annahme einer langen Bewahrung des Aoristes und seines Einflusses auf die Ausbildung des germ. Präteritums gerechtfertigt ist, wird uns in Kap. 3.1. in einem anderen Zusammenhang noch einmal beschäftigen. Für den Moment genügt die Feststellung, dass innerhalb der Verben mit Wurzelvokal *e die Reduplikationslosigkeit allmählich zum Normalfall wurde.

Die oben erwähnte grundsprachliche Alternation $*^{*}-{ }^{*} o-\neg$ tritt in Kl. I-III mit den lautgesetzlichen germ. Reflexen auf. Dagegen zeigen Kl. IV und V in Averbo 3 die Dehnstufe ${ }^{*} \bar{e}^{l}$. Tabelle 1 zeigt die lautgesetzliche Entwicklung der Stammformen, wie wir sie für das Vorgerm., ${ }^{9}$ das Frühgerm. vor der Dereduplikation (,germ. 1“) und für die Zeit danach (,germ. 2“) ansetzen. Nicht-lautgesetzliche Entwicklungen sind durch Unterstreichung markiert. ${ }^{10}$

\begin{tabular}{|c|c|c|c|c|}
\hline $\begin{array}{l}\text { Klasse (inkl. } \\
\text { Reihenmerkmal) }\end{array}$ & $\begin{array}{l}\text { Averbo } 1 \\
\text { (Inf., Präs.) }\end{array}$ & $\begin{array}{l}\text { Averbo } 2 \\
\text { (1.Sg.Prät.) }\end{array}$ & $\begin{array}{l}\text { Averbo } 3 \\
\text { (1.PI.Prät.) }\end{array}$ & $\begin{array}{l}\text { Averbo } 4 \\
\text { (Verbaladj.) }\end{array}$ \\
\hline I: vorgerm. & *réid ${ }^{\mathrm{h}}$-e/o- & *re-róid ${ }^{\mathrm{h}}-\mathrm{h}_{2} \mathrm{e}$ & *re-rid ${ }^{\mathrm{h}}$-mé & *rid ${ }^{\mathrm{h}}-(\mathrm{o})$ nó- \\
\hline germ. 1 & \multirow{2}{*}{$\begin{array}{l}\text { *rīđ-a- } \\
\text { 'reiten' }\end{array}$} & $*^{*}$ re-raiđ- ${ }^{\mathrm{a}}$ & ${ }^{*}$ re-riđ-um ${ }^{\mathrm{e}}$ & *riđ-ana-z \\
\hline germ. 2: *īK & & *raid & *riđ-um & *riđ-ana-z \\
\hline II: vorgerm. & *deuk-e/o- & *de-dóunk-h $\mathrm{h}_{2} \mathrm{e}$ & *de-duk-mé & *duk-(o)nó- \\
\hline germ. 1 & \multirow{2}{*}{$\begin{array}{l}\text { *teuh-a- } \\
\text { 'ziehen' }\end{array}$} & ${ }^{*}$ te-tauh $-{ }^{\mathrm{a}}$ & $*$ te-tugum ${ }^{\text {el1 }}$ & *tug-ana-z \\
\hline germ. 2: *euK & & $*_{\text {tauh }}$ & *tug-um & *tug-ana-z \\
\hline III: vorgerm. & $* \mathrm{~h}_{2}$ melg-e/o- & ${ }^{*} \mathrm{~h}_{2} \mathrm{e}-\mathrm{h}_{2} \mathrm{mó} \mathrm{lg}-\mathrm{h}_{2} \mathrm{e}$ & ${ }^{*} \mathrm{~h}_{2} \mathrm{e}-\mathrm{h}_{2} \mathrm{ml} \mathrm{g}$-mé & $* \mathrm{~h}_{2} \mathrm{ml} \hat{\mathrm{g}}-(\mathrm{o}) \mathrm{nó}-$ \\
\hline germ. 1 & \multirow{2}{*}{$\begin{array}{l}\text { *melk-a- } \\
\text { 'melken' }\end{array}$} & $*^{\text {me-malk- }}{ }^{\mathrm{a}}$ & *me-mulk-um & *mulk-ana-z \\
\hline germ. 2: *eRK $(* u R K)^{12}$ & & *malk & $*_{\text {mulk-um }}$ & *mulk-ana-z \\
\hline IV: vorgerm. & $* \mathrm{~b}^{\mathrm{h}} \mathrm{er}-\mathrm{e} / \mathrm{o}-$ & $* b^{\mathrm{h}} \mathrm{e}-\mathrm{b}^{\mathrm{h}}$ ór- $\mathrm{h}_{2} \mathrm{e}$ & ${ }^{*} \mathrm{~b}^{\mathrm{h}} \mathrm{e}-\mathrm{b}^{\mathrm{h}} \mathrm{r}-\mathrm{mé}$ & *b $\mathrm{b}_{\mathrm{h}}^{\mathrm{r}-(\mathrm{o}) \text { nó- }}$ \\
\hline germ. 1 & \multirow{2}{*}{$\begin{array}{l}\text { *ber-a- } \\
\text { 'tragen' }\end{array}$} & ${ }^{*}$ be-bar- ${ }^{\mathrm{a}}$ & *be-bur-um ${ }^{\mathrm{e}}$ & *bur-ana-z \\
\hline germ. 2: *e $\boldsymbol{R}(* u R)$ & & *bar & *bēr-um & *bur-ana-z \\
\hline V: vorgerm. & *ped-e/o- & *pe-pód-h $\mathrm{h}_{2} \mathrm{e}$ & *pe-pd-mé & *pd-(o)nó- \\
\hline germ. 1 & \multirow{2}{*}{$\begin{array}{l}* \text { fet-a- } \\
\text { 'fallen' }\end{array}$} & $*_{\text {fe-bat- }}{ }^{\mathrm{a}}$ & $*_{\mathrm{fe}} \mathrm{ft}-\mathrm{um}^{\mathrm{e}}$ & $*$ fet-ana- $z^{13}$ \\
\hline germ. 2: *eT $(* u T)$ & & $*_{\text {fat }}$ & *fêt-um & *fet-ana-z \\
\hline
\end{tabular}

Tabelle 1

9 „Vorgerm.“ Formen sind nur für Illustrationszwecke angesetzte Stammformen, die die lautliche Entwicklung der idg. Ablautalternationen zum Germ. hin veranschaulichen sollen. Lautlich zeigen sie noch keine germ. Charakteristika, die grundsprachlichen Restriktionen hinsichtlich der Bildung von Perfektstämmen (vgl. oben) sind jedoch schon gefallen. Außer für die Wurzel *ped- wird nämlich zu keinem der Verben ein grundsprachliches Perfekt angesetzt, vgl. Rix \& Kümmel (2001: 502, 124, 279, 76f., 458)!

10 Bei der Verschriftung der germ. Formen folge ich weitgehend den Konventionen des Etymological Dictionary of Proto-Germanic von Kroonen (2013), d.h. die Glides idg. $*^{*}, *_{i} i$ werden - sofern nicht Bestandteil eines Diphthongs - mit $w, j$, der bei der Ersten Lautverschiebung entstandene Frikativ germ. $* \chi$ $(<$ idg. $* k, * \hat{k})$ mit $h$ verschriftet. Abweichend von Kroonens Usus schreibe ich für den Labiovelar idg. $* g^{u}$ germ. $k^{w}$ (anstelle von $k w$ ), da es sich dabei eindeutig um ein Phonem handelt, vgl. dazu Braune \& Heidermanns (2004: §59). Auch werden Fortsetzer von idg. $* b^{h}, * d^{h}, *^{h}$ nicht einheitlich mit $b, d, g$ verschriftet, sondern je nach lautlicher Umgebung als Plosive $(b, d, g)$ oder Frikative $(\hbar, d, g)$. $\hbar, d$ und $g$ bezeichnen zudem die gemäß dem Verner'schen Gesetz stimmhaft gewordenen Kontinuanten von nachidg. $* p, * t$ und $* k(<\operatorname{idg} . * k, * \hat{k})$.

11 Germ.* ${ }^{*}<$ germ. * $\chi$ gemäß den Regeln des Verner'schen Gesetzes.

12 Verben mit Wurzelvokal germ. *u treten vereinzelt in den Kl. III-V auf, vgl. ae. murnan 'bangen, trauern, fürchten' oder got. trudan 'treten'. Zu *e/*u-Doubletten in Kl. IV und V vgl. auch das in Kap. 5 Gesagte.

13 Entweder sind die $e$-stufigen Formen von Averbo 4 nach dem Vorbild von K1. VI und VII gebildet, wo Averbo 1 und 4 denselben Vokalismus zeigen, oder es kam zu einer Vokalepenthese. Falls Letzteres der Fall war, kannte vielleicht auch Averbo 3 zwischenzeitlich eine e-Stufe (*fetum?). Diese Form hätte aber kaum lange überlebt, weil die Tempuskategorie in germ. starken Verben mit Wurzelvokal *e immer auch durch Stammmodulation markiert wird, vgl. die Besprechung des Beispielparadigmas in diesem Kap. sowie Kap. 5. 
$\mathrm{Ab}$ der hier „Germ. 2“ genannten Sprachstufe entscheidet der Wurzelreim über die Klassenzugehörigkeit eines Verbs (er fungiert daher auch als sog. Reihenmerkmal). Davor galt wohl ungeachtet der Wurzelstruktur die Alternation $* e-{ }^{*} o-\neg$.

Wie das Averbo 4 von *ber- $a$ - zeigt, wäre eine Schwundstufe gemäß dem Muster $* e-{ }^{*} o$ - $\neg$ im Prät.Pl. von Verben mit der Wurzelstruktur *eR ohne weiteres möglich gewesen. Wahrscheinlich existierte sie auch einst (*burum), da ein gemäß den idg. Sonantisierungsregeln silbischer Resonant in einer vorgerm. schwundstufigen Form $* b^{h} e-b^{h} r$-mé lautgesetzlich zu germ. *-ur- aufgefaltet wurde. Auch zeigen die germ. Präteritopräsentien mit der Wurzelstruktur $* K_{0} V R-$ - formal meist Fortsetzer indogermanischer Perfekta und mit präsentischer Bedeutung - schwundstufigen Pluralvokalismus: germ. *skal - *skulum 'sollen' und *man * munum 'meint/meinen; erinnert/erinnern sich'. Daher wird die Dehnstufe in Kl. IV aus gutem Grund ausnahmslos als jüngere Analogieerscheinung nach dem Muster von K1. V erklärt, vgl. Matzel (1970: 178), Bammesberger (1986: 55), Schumacher (2005: 603) und Mailhammer (2007: 67, mit Lit.). Die Bewahrung der ursprünglichen Ablautverhältnisse in den Präteritopräsentien ist sicherlich vor dem Hintergrund zu sehen, dass die Alternation * $a-$ $* u$ hier im Präsens auftrat und dem Analogievorgang daher nicht ausgesetzt war, vgl. Matzel (1970: 175).

Für die Erklärung der Dehnstufe können wir uns also auf Kl. V konzentrieren. Betrachten wir dazu Averbo 3 eines Verbs wie germ. *fet-a- (zur Wurzel *ped- 'treten; fallen, sinken') in Tabelle 1: Solange die germanischen Präterita reduplizierten, ergab sich durch die dem Stamm vorausgehende Reduplikationssilbe eine unproblematische Silbenstruktur, vgl. frühgerm. *fef.tum 'wir fielen' neben dem Sg. *fe.bat. Ein einfacher Reduplikationsabbau, wie wir ihn z.B. für germ. ${ }^{*}$ te-tauh ${ }^{a},{ }^{*}$ te-tugum ${ }^{e}>$ germ. ${ }^{*}$ tauh, ${ }^{*}$ tugum annehmen können, war in Verben von K1. V ausgeschlossen, da dem Stamm ein Silbenkern gefehlt hätte (†ft-um). Drehund Angelpunkt der Auseinandersetzung mit der Averbostufe 3 von Kl. V ist nun die Frage, wie die Ersetzung der redupliziert-schwundstufigen Formen mit deredupliziert-dehnstufigen abgelaufen ist. Dies wird uns im folgenden Kapitel beschäftigen.

Bevor wir uns diesem Problem zuwenden, ist es im Hinblick auf die weitere Diskussion sinnvoll, die Kategorien des germ. starken Verbs und deren Markertypen zu betrachten. Zur Veranschaulichung dient das Paradigma von germ. *werp- $a$ - 'werden' (Sprachstufe „Germ. 2“; Formen nach Ringe 2006: 265f.):

\begin{tabular}{|c|c|c|c|c|}
\hline & Präs.Ind.Akt. & Präs.Opt.Akt. & Prät.Ind.Akt. & Prät.Opt.Akt. \\
\hline 1.Sg. & $*_{\text {werp-ō }}$ & $*_{\text {werp-au }}$ & $*_{\text {warp }}$ & *wurd-iju (?) \\
\hline 2.Sg. & ${ }^{*}$ wirp-izi ${ }^{14}$ & *werp-aiz & ${ }^{*}$ war-st ${ }^{15}$ & *wurd-īz \\
\hline 3.Sg. & *wirp-iđi & *werp-ai & $*_{\text {warb }}$ & *wurd-1̄ \\
\hline 1.Dual & *werb-ōz (?) & *werp-aiw & ${ }^{*}$ wurd-ū $(?)^{16}$ & ${ }^{*}$ wurd-1̄w \\
\hline 2.Dual & *werb-ađiz (?) & $*_{\text {werb-aiđiz (?) }}$ & *wurd-uđiz (?) & *wurd-īđiz (?) \\
\hline 1.Pl. & *werp-amaz & *werp-aim & *wurđ-um & *wurđ-īm \\
\hline 2.Pl. & *wirp-iđ & *werp-aiđ & *wurđ-uđ & *wurđ-īđ \\
\hline 3.Pl. & *werp-anđi & $*_{\text {werb-ain }}$ & $*_{\text {wurd-un }}$ & *wurd-īn \\
\hline
\end{tabular}

Tabelle 2

14 Die $* i$-haltigen Formen im Präs. sind das Resultat einer Hebung $*_{e}>*_{i} /{ }_{-} \$ *_{i}$.

15 Das Flexiv der 2.Sg.Prät. war *-t, vgl. got. nam- $t$ 'du nahmst'. Beim Aufeinandertreffen zweier Dentale, hier vom Stammauslaut und dem Flexiv (*warp- $t$ ), entstand im Germ. lautgesetzlich ein Sibilant. Interparadigmatische Ähnlichkeit wurde in diesen Fällen durch analogisches *-st wiederhergestellt, vgl. got. waist ( ${ }^{*}$ waiss), vgl. Braune \& Heidermanns (2004: §81).

16 Die Stimmhaftigkeit des wurzelschließenden Konsonanten in dieser und den folgenden Paradigmenzellen ist durch den idg. Akzentsitz (ursprünglich auf der Endung) bedingt (Verner'sches Gesetz). 
Die formal markierten Kategorien Tempus (mit den beiden Werten Präs. vs. Prät.), Modus (Ind. vs. Opt. vs. der hier ausgeklammerte Imperativ), Numerus (Sg. vs. Dual vs. Pl.) und Person werden mit unterschiedlichen Verfahren markiert, nämlich mittels Stammmodulation und Suffixen. ${ }^{17}$ Wenden wir uns zuerst dem stammmodulativen Verfahren Ablaut zu: Dieser ist in erster Linie ein eindeutiger Marker für die Kategorie Tempus, indem die $e$-Stufe (und ihre Allophone) nur in präsentischen Paradigmenzellen auftritt, die übrigen nur in präteritalen. Ferner symbolisiert er im Prät.Ind. die Opposition Sg. vs. Dual/Pl. Im Opt. dagegen charakterisiert der Ablaut nur Tempus, zur Numerusunterscheidung dient er nicht. Als zweiter Markertyp treten die Ausgänge ${ }^{18}$ auf, die jede Paradigmenzelle hinsichtlich der Kategorien Modus, Numerus und Person spezifizieren. Zusätzlich tragen sie zusammen mit dem Ablaut die Tempusmarkierung, wodurch dieser Kategorie doppelte Exponenz zukommt.

Insgesamt ergibt sich ein wenig erstaunliches Bild der Morphologie des germ. starken Verbs: Die im Sinne Bybees (1985: 13-24) hochrelevante Tempuskategorie wird durch ein stammmodulatives und ein additives Verfahren markiert. Ähnlich liegen die Verhältnisse in der Numeruskategorie, wo durch unterschiedliche Ablautstufen zumindest im Prät. der Verben mit Wurzelvokal *e eine strikte Trennung zwischen den Stämmen des Singulars und des Nicht-Singulars besteht.

\section{Erklärungsansätze}

Im Folgenden werden drei unterschiedliche Ansätze zur Herleitung von germ. ${ }^{*} e^{-1}$ präsentiert: Die Aoristhypothese, die sog. „bigêtun-Regel“" und die Analogiehypothese. Die Besprechung dieser Theorien fällt relativ umfassend aus. Diese Fokussetzung ist erforderlich, weil die Aoristhypothese und die ,bigêtun-Regel“ unmittelbar mit zwei vielbeachteten Forschungsfragen verquickt sind:

1) Die Aoristhypothese zur Erklärung von $*^{-}{ }^{l}$ in K1. IV und V steht im Kontext der Diskussion, welche Spuren der (nach-)idg. Aorist im germ. Verbalsystem hinterlassen hat. Nachdem sich die Forschung in den letzten Jahrzehnten auf den Konsens verständigt hat, dass nur einige wenige idg. Präsensbildungen sowie das idg. Perfekt am Aufbau des germ. Verbalsystems beteiligt gewesen seien, haben die Beiträge Kortlandts (zuletzt 2011) und Mottauschs (2013) die Diskussion um eine Einmischung des Aoristes neu entfacht, vgl. auch Kap. 2 oben. Ihre Thesen sind prüfenswert und müssen daher auch bei der Diskussion um die Ursprünge von $*^{-1}$ als Marker des Prät.Pl. berücksichtigt werden.

2) Mit der „bigètun-Regel“ (Schumacher 2005) und ähnlichen Ansätzen wird versucht, die Dehnstufe im Prät.Pl. der Kl. IV und V mit phonologischen Constraints des Idg. in Verbindung zu bringen. Die germ. Dehnstufenpräterita seien daher direkt mit dehnstufigen Perfekta in anderen Subphyla zu vergleichen. Ansätze, die die Ursache einer in vielen Tochtersprachen wiederkehrenden Auffälligkeit in der Grundsprache sehen, haben den Vorteil, dass zumindest sprachvergleichend gesehen weniger Annahmen für die

17 Das idg. Perfekt (formal: $\rightarrow$ germ. Präteritum) kannte von alters her keine Diathesenopposition (vgl. Ringe 2006: 26); sie wird daher im Folgenden nicht berücksichtigt.

18 Die Segmentierung von Stammbildungselement und Endung ist bereits im Germ. infolge Lautwandels nicht immer ohne weiteres möglich. Der Einfachheit halber wird daher häufig darauf verzichtet und die beiden Einheiten unter dem Begriff „Ausgang“ subsumiert. 
Lösung scheinbar ähnlich gelagerter Problemfälle bemüht werden müssen (entia non sunt multiplicanda praeter necessitatem). Nimmt man Ockhams Rasiermesser als Maxime ernst, ist eine Erklärung, die ,auf einen Schlag“ Rätsel verschiedener Einzelphilologien zu lösen vorgibt, einer gründlichen Überprüfung zu unterziehen. Gegenüber mehreren einzelsprachlichen Lösungen ist ihr aber natürlich nur dann der Vorzug zu geben, wenn ihre Grundannahmen Bestätigung in der einzelsprachlichen Evidenz finden.

\subsection{Die Aoristhypothese}

Ein u.a. von Cowgill (1957: 46-56) beschriebener Ansatz weitet die Suche nach möglichen Quellen der Dehnstufe auf das idg. Stammbildungsinventar aus. In den Blickpunkt rückt dabei besonders der Aorist, der bei als abgeschlossen aufgefassten Verbalhandlungen Verwendung fand und daher beim Übergang von einem Aspekt- zu einem Tempussystem ein Vergangenheitstempus im eigentlichen Sinne werden konnte (vgl. auch den Synkretismus von idg. Perfekt und Aorist beim Aufbau des ital. Perfekts und des kelt. Präteritums!). Darüber hinaus besteht mit dem sigmatischen Aorist eine Stammbildung, in der der starke Stamm dehnstufigen Vokalismus zeigt, vgl. zur Wurzel *deuk- 'ziehen' den $s$-Aorist *déuk-s-/*déuk-s- (> lat. $d \bar{u} x \bar{l}$ ), vgl. Rix \& Kümmel (2001: 124). Es ist zudem wahrscheinlich, dass Aoriststämme, die im Idg. wohl weitaus zahlreicher waren als Perfektstämme, ${ }^{19}$ im germanischen Präteritum Spuren hinterlassen haben (Mottausch 2013, pace Ringe 2006: 157, Mailhammer 2007: 39f.), vgl. dazu auch 2. zu den möglichen Ursachen der Perfektdereduplikation im Germ.

Mailhammer (2007: 76) verwirft den Ansatz eines Aoristeinflusses auf das sich herausbildende germ. Prät. unter Verweis auf das Wirken des Verner'schen Gesetzes, das auch in Verben von K1. V klar sichtbar ist (vgl. das von ihm angeführte Beispiel des ahd. Verbs wësan 'sein' mit seinen Averbos 2 und 3, was - wārun, < germ. *was - *wēzun) und einen ursprünglichen Akzentwechsel zwischen dem Stamm (im Sg.) und den Endungen (im Pl.) voraussetzt, wie er für das idg. Perfekt zweifelsfrei rekonstruiert wird. Ein solcher Wechsel fehlte dem akrodynamisch flektierten $s$-Aorist. Dieser Argumentation könnte man entgegenhalten, dass sich der Dehnstufenvokal ausgehend von einigen vor- oder frühgerm. Aoristformen zu Wurzeln hätte ausbreiten können, deren wurzelschließender Konsonant ohnehin keiner akzentbedingten Stimmhaftigkeitsalternation unterworfen gewesen wäre (d.h. mit wurzelschließendem idg. $* b^{h}, * d^{h},{ }^{*} g^{h}, * b,{ }^{*} d,{ }^{*} g$ ), wie z.B. im Falle von $* g^{u h} e d^{h}$ - 'bitten, wünschen' (> germ. *bid-ja-) mit dem $s$-Aorist ${ }^{*} g^{u h} \frac{\bar{e}}{d^{h}}{ }^{-}-{ }^{-}{ }^{*}{ }^{*} g^{u h} \dot{e} d^{h}-s-$ (Ansatz nach Rix \& Kümmel 2001: 217). Die Dehnstufe des Singulars wäre später im gesamten Aoristparadigma verallgemeinert worden (Analoga bietet die lateinische Sprachgeschichte, vgl. Kim 2001: 136). Ein solches, durchgehend dehnstufiges Paradigma wäre in der Folge beim Zusammenfließen von Perfekt und Aorist im germanischen Präteritum zusammen mit Formen eines vor- oder frühgerm. Neoperfekts zu einem neuen Paradigma kombiniert worden. Dabei stellte die starke Alternante des Neoperfekts $* g^{u h} e-g^{u h} o d^{h}$ - die Formen von Averbo 2, der Aorist $* g^{u h} \frac{\dot{e}}{e} d^{h}-s-$ diejenigen von Averbo 3. Es ist in diesem Szenario aber nicht ersichtlich, wie die Tilgung des

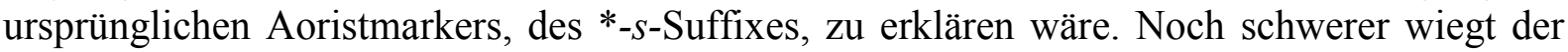
Umstand, dass für das Germ. keine sicheren Reflexe von $s$-Aoristen vorliegen, vgl. Mottausch (2000: 46). Den fünf von Kortlandt (2011: 147) unter Vorbehalt angeführten Beispielen von Verben, in denen der einstige Aspektmarker $*_{-s-}$ wurzelhaft geworden sein soll, fehlen im Falle von germ. *blēs- $a$ - 'blasen', *leus- $a$ - 'verlieren' und *bins- $a$ - 'ziehen' außergerm.

19 Schumacher (2005: 591) zählt auf Basis des Lexikons der indogermanischen Verben (Rix \& Kümmel 2001) 604 Aoriststämme gegenüber 281 Perfektstämmen. 
bezeugte Aoristformen. Zu den Etyma von *hreus- $a$ - 'fallen' und *geus- $a$ - 'sprudeln ${ }^{, 20}$ liegen nicht einmal außergerm. Komparanda vor, die eine Etymologisierung und somit eine Entscheidung über den ursprünglichen Status von $*_{-s-}$ ermöglichen würden. Eine vermeintliche Alternative zur problematischen Herleitung aus dem sigmatischen Aorist ist diejenige aus einem Wurzelaorist, wenn man entgegen der communis opinio für diesen mit Kortlandt (2004) anstatt Normalstufe ebenfalls ursprüngliche Dehnstufe ansetzt. Der von ihm angeführte Kronzeuge für das Postulat eines dehnstufigen Wurzelaorists, lat. vēnit, got. qêmum, Toch. B sém, die er auf einen idg. dehnstufigen Wurzelaorist idg. * $g^{u} \bar{e} m t$ ' kam' zurückführen möchte, ist aber wohl der lautgesetzliche Fortsetzer eines normalstufigen Wurzelaorists 1.Sg. idg. ${ }^{*} g^{u} e ́ m-m$ (> idg. ${ }^{*} g^{u} \bar{e} m$ durch das Wirken von Stangs Gesetz, vgl. Kim 2001: 125, zustimmend Jasanoff 2012: 127, Fn. 2).

Angesichts der vielen ad hoc-Annahmen, die für das Postulat eines aoristischen Ursprungs der Dehnstufe in germ. Präterita nötig würden, kann die Aoristhypothese kaum eine plausible Lösung für unser Problem sein. Sie wird daher an dieser Stelle nicht weiterverfolgt.

\subsection{Die Ersatzdehnungshypothese oder „,bigētun-Regel““(Schumacher 2005)}

Eine vielfach beschriebene, im Detail jeweils leicht unterschiedlich konfigurierte Hypothese sieht im dehnstufigen Vokal das Resultat einer Ersatzdehnung in der schwundstufigen Pluralalternante eines idg. Perfekts durch die Vereinfachung des Obstruentenclusters der Stammsilbe, schematisiert ${ }^{*} T_{1} e-T_{1} T_{2^{-}}>* T_{1} \bar{e} T_{2^{-}}$, so bereits Streitberg (1974 [1896]: §96, allerdings noch breiter und auch für Verben mit wurzelschließendem Resonanten formuliert), zuletzt Schumacher (2005). Letzterer beschreibt den vermeintlichen Wandel als eine „morphonologische Regel“" und nennt sie - nach einer angeblich so entstandenen Prät.Pl.Alternante des Got. - „bigētun-Regel“. Angesichts der oben angeführten (hypothetischen), phonotaktisch unproblematischen Vorform * feftum stellt sich die Frage nach der Motivation einer solchen Regel. Es ist Schumachers (2005: 601f.) Verdienst, dass er den postulierten Lautwandel - der andernorts nolens volens angenommen wurde - zu begründen versucht. Dazu rekurriert er auf die phonotaktischen Constraints, die die grundsprachliche Wurzelstruktur beeinflusst haben (vgl. dazu u.a. Ringe 1998: 174-176). Zu diesen zählt die Regel, dass ,,[e]ine uridg. Wurzel [...] nicht ein und denselben Obstruenten beiderseits des Vollstufenvokals * $e$ haben“ könne (Schumacher 2005: 601). Diese Beschränkung, so Schumacher, habe sich auch bei der Bildung von schwundstufigen Perf.Pl.-Alternanten zu Wurzeln der Struktur ${ }^{*} T_{1} e T_{2^{-}}$geltend gemacht, in denen der Reduplikationsvokal gemäß den Bildungsregeln zwischen denselben tautosyllabischen Obstruenten stand. Die bigètun-Regel ${ }^{*} T_{1} e-T_{1} \cdot T_{2^{-}}>* T_{1} \bar{e} T_{2^{-}}$wäre daher nichts anderes als das Resultat einer phonotaktischen Restriktion, deren Domäne die Indogermanistik bis anhin zu eng definiert hat. Zur Rechtfertigung der Annahme, dass die bzgl. der grundsprachlichen Wurzelstruktur geltenden Verhältnisse eins zu eins auf die Bildung von Perfektstämmen übertragen werden dürften, argumentiert Schumacher (ibid.),

dass im Perfekt besondere strukturelle Regeln gelten. Obwohl es unbestreitbar ist, dass im Perfekt zwischen Reduplikationssilbe und Wurzelsilbe eine Morphemfuge vorliegt, ist diese Morphemfuge von einer besonderen Qualität. Alle anderen Morphemfugen des Urindoger-

20 Die Kürze des Wurzelvokals in *leus-a- und *bins-a-, *hreus- $a$ - und *geus-a- stellt aber nicht per se ein Problem dar, da idg. Langvokale vor *RT-Clustern (hier hypothetisch idg. *-ênus- resp. *-ēns-) gekürzt wurden, vgl. zu diesem „Osthoffkürzung“ genannten Lautgesetz etwa Müller (2007: 45). 
manischen verlaufen nämlich entweder zwischen Wurzel und Suffix oder zwischen Kompositionsvorderglied und Kompositionshinterglied. Da aber die Reduplikationssilbe aus der Wurzel heraus generiert ist, haben wir es hier gewissermaßen mit einer Extension der Wurzel selbst zu tun; und möglicherweise gelten in diesem ganz besonderen morphologischen Kontext besondere strukturelle Bedingungen, die eng verwandt sind mit den Regeln, die die grundsprachliche Wurzelstruktur an sich betreffen.

Den regelhaften Output der bigētun-Regel sieht Schumacher (2005: 603) in *gètun 'sie bekamen', *fētun 'sie fielen' und *beggun 'sie empfingen, nahmen' sowie womöglich auch in * $k^{w} \bar{e}$ đun 'sie sagten' und *gētun 'sie gaben', da ein grundsprachliches Perfekt hier in diesen Fällen „wahrscheinlich bzw. durch Gleichungen nachweisbar“ sei. Von diesen mind. drei bis fünf Verben - man ergänze *et-a-, vgl. 3.3. - habe sich $*^{-1}$ bei der Bildung der zahlreichen Neoperfekta des Germ. analogisch ausgebreitet.

Schumachers Postulat eines „,besonderen morphologischen Kontext[s]“ ist zwar ad hoc, aber nicht per se widerlegbar. Es spricht dennoch zweierlei gegen diese Motivierung der bigētun-Regel: Erstens müsste im beschriebenen „Kontext“" auch in anderen Stammbildungen eine Kontraktion erfolgt sein, in denen gemäß der jeweiligen Bildungsregel $* T_{1} e-T_{1} T_{2^{-}}$ Strukturen entstanden, d.h. in reduplizierten Präsentien und Aoristen (womöglich auch in Intensiva, sofern man sie für die Grundsprache ansetzt), im Falle derer ein Bestandteil des Stammes also ,,aus der Wurzel heraus generiert ist“. Selbst wenn man mit Schumacher annimmt, es handle sich bei der bigētun-Regel um eine „morphonologische Regel“, die auf die Perfektbildung beschränkt gewesen sein könnte, müssten in anderen Kontexten zumindest Spuren einer solchen Kontraktion zu finden sein, denn „,[e]ven morphologized phonological processes normally begin as Neogrammarian sound laws“, wie es Jasanoff (2012: 128) formuliert. Reduplizierte Präsensbildungen zeigen aber Erhalt des inlautenden ${ }^{*} T_{1} T_{2}$-Clusters, vgl. das Präsenspartizip ved. píbdamāna- 'abwechselnd fest auftretend', vgl. Rix \& Kümmel (2001: 458). Zweitens, und dies widerlegt die bigètun-Regel endgültig, gibt es, wie Jasanoff (2012: 128, Fn. 5) und Bammesberger (1996: 36) bemerken, durchaus auch Perf.Pl.-Alternanten mit erhaltener $* T_{1} e-T_{1} T_{2}$-Struktur oder direkten Reflexen derselben, nämlich u.a. ved. 3. P1. ni-ședúh 'sie haben sich niedergelassen', avest. hazd- <*sa-zd-< idg. *se-sd- zur Wurzel *sed- 'sich setzen'(vgl. Kümmel 2000: 543f., 676; Rix \& Kümmel 2001: 513, 515, Fn. 18; zum Lautlichen s. auch Kap. 4). Somit scheint auch der Ansatz, den Dehnstufenvokal in Kl. IV und V des germ. starken Verbs mittels einer phonologischen (ggf. später morphologisierten) Regel der Grundsprache zu gewinnen, gescheitert.

\subsection{Die Analogiehypothese}

Dieser ebenfalls schon mehrfach vorgeschlagene Lösungsansatz sieht den Ursprung des germ. Dehnstufenvokals meist in einem Verb, nämlich dem ESSEN-Wort aus der Wurzel idg. * $h_{1} e d-$ (vgl. u.a. lat. edo 'ich esse', heth. èdmi, adanzi , 'essen', ved. átti, adánti 'id.', air. ithid 'isst', Rix \& Kümmel 2001: 230), zu der im Germ. allem Anschein nach ein reguläres thematisches Präsens *et-a- vorlag. Zu dieser Wurzel wird auch eine grundsprachliche Perfektstammbildung angesetzt, nämlich * $h_{1} e-h_{1} o^{\prime} d-{ }^{*} h_{1} e-h_{1} d{ }^{\prime}$ (vgl. aus dem Außergerm. lat. $\bar{e} d \bar{l}$, air. dúaid

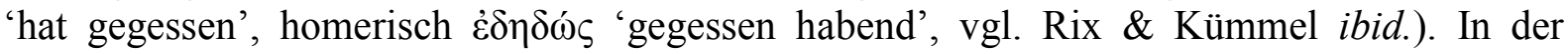
schwachen Alternante dieses Perfekts sei durch Laryngalwandel im Germ. auf direktem, d.h. lautgesetzlichem Weg eine dehnstufige Form * $\bar{e} t$ - entstanden. In der Folge sei die Alternation zwischen Averbo $1 * e t$ - und Averbo $3 * \bar{e} t$ - analogisch auf alle anderen Verben mit Wurzelstruktur $* K_{0} e T$ - übertragen worden, vgl. Mottausch (2000) oder Ringe (2006: 185-187). 
In der Forschung wurde wiederholt Skepsis gegenüber dem Szenario einer analogischen Ausbreitung ausgehend von einer so schmalen Basis geäußert, vgl. etwa Kortlandt (1992: 103) oder Müller (2007: 172). Diese Problematik wird in Kap. 4 ausführlicher diskutiert. Für die weitere Diskussion müssen wir zuerst das mögliche Musterverb und seine Stammformen eingehender betrachten. Der einzelsprachliche Befund lässt bei Berücksichtigung der jeweiligen Lautgesetze eine unproblematische Rekonstruktion der gemeingerm. Averbostufen zu. Es sind dies neben dem Infinitiv im Averbo 2 *êt- (vgl. 1./3.Sg.Prät.Ind. got. fr-êt 'fraß', ahd. $\bar{a} 3$, awn. át, ae. $\bar{e} t$ ), im Averbo 3 *êt- (vgl. 3.Pl.Prät.Ind. got. êtun, ahd. $\bar{a} 3(3) u n$, awn. ótu, ae. äton) und im Averbo 4 *et-i/anaz (> ahd. gi(g)-ezzan, awn. etinn, ae. eten), vgl. Seebold (1970: 179), zu fr-êt vgl. Braune \& Heidermanns (2004: §176, Anm. 3). Das Verb gehört mit seiner Wurzelstruktur $K_{0} e T$ - zur Klasse $\mathrm{V}$ der germanischen starken Verben, doch fällt es aufgrund der Dehnstufe im Averbo 2 aus dem Rahmen. Hier wäre, sofern man ein grundsprachliches Perfekt voraussetzt, vielmehr eine idg. o-Stufe zu erwarten, d.h. †(e-)át- als Kontinuante von $* h_{1} e-h_{1} o ́ d-$, vgl. 2. Zwar lässt sich den Schriften Otfrids und im Mhd. a3 als Prät.Sg. nachweisen, doch wird dies gemeinhin als jüngere Form beurteilt, die sekundär nach dem üblichen Muster von Kl. V umgebildet wurde, vgl. Braune \& Reiffenstein (2004: §343, Anm. 5). Ein mögliches Argument für die einstige Existenz von germ. $\dagger(e-) a ́ t$ könnten Ableitungen zur Verbalwurzel liefern: Mehrere Einzelsprachen führen eine germ. Kausativbildung germ. *at-ja- fort (Ursprungsbed. wohl 'veranlassen, dass jemand isst'), vgl. got. fra-atjan* 'zum Essen austeilen', awn. etja 'essen lassen, füttern', ae. ettan 'weiden' (Schnieders 1938: 12), ahd. etzen 'abweiden, abfressen lassen; ätzen' (Lloyd et al. 1998: 1188). Solche Kausativbildungen zu starken Verben reflektieren ein idg. Wortbildungsmuster, das durch $o$-Stufe und das Suffix *-éie/o- charakterisiert war (vgl. beispielsweise Rix \& Kümmel 2001: 22). In Darstellungen zur Bildung germanischer Kausativa wird häufig darauf hingewiesen, dass im Germanischen synchron betrachtet stets die Averbostufe 2 des zugrundeliegenden starken Verbs als Ableitungsbasis dient, vgl. etwa Krause (1968: 238). Folglich müssen germ. Kausativa entgegen dem idg. Muster nicht zwingend $o$-stufigen Vokalismus zeigen, vgl. das germ. Kausativum *fōr-ija- 'führen', das vom Averbo 2 von *far- $a$ - 'gehen' gebildet wurde (anstelle von †far-ja-). Diese Reanalyse des grundsprachlichen Derivationsmusters der Kausativa erfolgte, wie García García (2005: 17) beschreibt, aufgrund der gemeinsamen Abtönstufe (idg. ${ }^{*} o>$ germ. ${ }^{*} a$ ) des idg. Bildungsmusters einerseits und von Stammform 2 in Verben der Kl. I-V andererseits. Die derivationelle Koppelung von Averbo 2 und der Kausativbildung sei dabei „rigide“ (ibid., Fn. 6), wie sie unter Verweis auf die Bildung ahd. āzen ‘jemandem zu essen geben, speisen, füttern' ausführt (< germ. *ēt-ija-): „, sie weist den Langvokal des Prät.Sg. des Grundverbs germ. *eta- 'essen' auf, obwohl er in der V. Klasse eine Ausnahme darstellt.“ Zwar behandelt García García auch ahd. etzen und stimmt der Rekonstruktion von germ. *at-ja- zu (2005: 55), doch äußert sie sich nicht zu den Implikationen des vermeintlichen Nebeneinanders der Kausativa germ. *ét-ija- und *at-ja-. Auffällig ist, dass äzen (mit der vermutlich obdt. Nebenform gi-ätzen) erst in spätahd. Quellen entgegentritt, vgl. Lloyd et al. (1998: 1188) und keine Entsprechungen in den anderen Einzelsprachen zu kennen scheint. Wie ist diese scheinbar widersprüchliche Faktenlage zu deuten? Eine Möglichkeit wäre, in *at-ja- einen Beweis für ein ursprüngliches Averbo 2 †e-át zu sehen; ahd. $\bar{a} z e n / g i-\bar{a} t z e n$ wäre folglich eine jüngere Bildung aus einer Zeit, in der *e-át bereits durch *êt ersetzt worden war. Es scheint indes nicht ratsam, hinter den germ. Kausativa eine einzige, einheitliche Wortbildungsschicht zu sehen. Vielmehr ist mit einer erst allmählichen Verfestigung der Koppelung von Averbo 2 und Kausativbildung im Laufe der germ. Sprachgeschichte zu rechnen. So kann *at-ja- ohne weiteres ein sehr altes Kausativum sein, dessen Bildung noch dem grundsprachlichen Muster folgte. Dem Ansatz eines germ. † (e-)át- fehlt 
also eine sichere Grundlage, und $* \bar{e} t$ - scheint die einzige legitime Form von Averbo 2 zu sein. Die Übereinstimmung im Stammvokalismus mit Averbo 3 ist in dieser Klasse isoliert, die Alternation $* e-* a-* \bar{e}^{-1}-{ }^{*} e$ ist in dieser mitgliederstarken Klasse sonst völlig fest. ${ }^{21}$ Die Forschung hat diese Abweichung unterschiedlich erklärt. Prokosch (1939: 172) mutmaßt, der Vokalismus des Prät.Pl. sei in den Sg. verallgemeinert worden, „on account of the ,unsubstantial " character of *at." Kortlandt (1992: 102f.) und Schumacher (1998: 187) weisen diese Erklärung als nicht stichhaltig ab, weil sie die Ersetzung einer regelmäßigen und eindeutigen Form, „,die nicht in stärkerem Maße als der Präsensstamm selbst ,unsubstantial ' gewesen wäre" (Schumacher: ibid.), für unwahrscheinlich halten. Stattdessen rechnet Schumacher (1998: 186f.) mit einer Kontraktion des alten $* e-a t,{ }^{22}$ die wahrscheinlich $* \bar{o} t$ ergeben haben müsse. Wie er selbst anmerkt, liefert das Germanische kein Parallelbeispiel für eine Kontraktion von $* e-a$-, weswegen über die genaue Qualität des dabei resultierenden Langvokals nur spekuliert werden kann. Die Alternation $* e t-a--{ }^{*} \bar{o} t-{ }^{*} \bar{e} t u n-{ }^{*} e t-i / a n a z$ sei aufgrund ihrer Isoliertheit im System anfällig für eine analogische Umgestaltung nach dem Vorbild von K1. VI (vgl. *ak-a- 'fahren' - * $\bar{o} k-{ }^{*} \bar{o} k u m-{ }^{*} a k$-i/anaz) gewesen, in der viele Verben eine mit *et- vergleichbare Wurzelstruktur $V K$ - zeigen. Gegen ein solches Szenario spricht, dass das Germanische vergleichbare Hiatus bei anderen Verben toleriert zu haben scheint, wurde doch in den Verben von Kl. VII ein durch Laryngalwandel lautgesetzlich geschwundener Reduplikationsvokal als Tempusmarker analogisch wieder eingeführt, worauf Schumacher (2005: 595f., 1998: 186, Fn. 15) selbst hinweist. ${ }^{23}$ Ferner bleibt unklar, weshalb nicht die angebliche Singularalternante $* \bar{o} t$ in den Plural verallgemeinert wurde, was aufgrund der vermutlich höheren Kategorienfrequenz des Singulars gegenüber dem Plural sowie der Übereinstimmung mit dem vermeintlichen Vorbild, dem Präteritalvokal von K1. VI ( $\left.{ }^{*} \bar{o} k-{ }^{*} \bar{o} k u m\right)$, zu erwarten gewesen wäre. Kaum überzeugender argumentiert Mottausch (1994: 135): Ihm zufolge habe die Reduplikationslosigkeit der Pluralalternante (in seiner Notation *étum) im Singular eine Umakzentuierung von *e-át- zu $* \dot{e}$-at bewirkt und $* \dot{e}-a$ - sei schließlich zu $* \bar{e}^{1}$ kontrahiert worden. Für die Bildung von Prät.Sg.-Formen des Musters * $(K) e-(K) a ́ K-$ zu Verben mit Infinitivvokal germ. *e lagen aber mindestens hunderte von Vorbildern vor, ${ }^{24}$ es ist daher nicht ersichtlich, weshalb sich †e-át nicht wie alle anderen Prät.Sg.-Formen mit *aVokalismus entwickeln und später deredupliziert als †at- hätte erscheinen können. Damit soll natürlich nicht impliziert werden, einem produktiven Muster folgende Verben könnten nicht irregularisiert werden - aus der Geschichte der germ. Einzelsprachen sind hinreichend Fälle bekannt, in denen Verben großer, produktiver Flexionsklassen unter Hochfrequenz Idio-

21 Matzel (1970: 176-178) verweist auf die vereinzelten dehnstufigen Formen des Prät.Sg. zum Verb *sit-ja'sitzen' im Ahd., As., Ae. und Awn., und verbindet die daraus abgeleiteten germ. Averbostufen *sit-ja- *sēt - *sētum mit lat. sedère - sēdī-sēdimus. Diese Gleichung sei ein Indiz für eine zweite grundsprachliche Perfektbildung, die sich gegenüber der in 2. beschriebenen durch das Fehlen der Reduplikation und eine durchgehende Dehnstufe ausgezeichnet habe. Diese Hypothese, die in unterschiedlichen Konfigurationen von Brugmann (1913: 183-187) über Meid (1971: 52-54) bis Jasanoff (2012, Imperfekta zu Narten-Präsentien) allenthalben vorgetragen wird - teils unter Einbezug weiterer dehnstufiger Vergangenheitsformen v.a. des Germanischen (starke Verben Kl. V), Italischen, Baltischen, Albanischen und Tocharischen - krankt aber an der Tatsache, dass zumindest für die beiden vermeintlichen „Bollwerke“ dieser Stammbildung, das Germ. und das Ital., entweder andere, weitgehend unumstrittene Quellen (Lat., vgl. Kim 2001: 135) plausibel sind oder im Vergangenheitstempus gar keine durchgehende Dehnstufe vorliegt, sondern Ablaut $* a-*_{\bar{e}}(\mathrm{Germ}$., vgl. das stets auf germ. * $a$ zurückweisende Averbo 2 des Got.).

22 Er äußert sich dabei nicht zur Betonung in reduplizierten Präterita; vgl. aber dazu das in Kap. 2 Gesagte, inkl. Fn. 8.

23 Vgl. nochmals das von ihm angeführte Beispiel des Verbs *auk-a- 'vergrößern, mehren' mit dem ererbten Perfekt (Präteritum) idg. ${ }^{*} h_{2} e-h_{2}$ óug- $>$ germ. $* a u k-\rightarrow$ germ. $* e$-auk- (woraus got. (ana-)aí-auk).

24 Vgl. Wegera \& Waldenberger (2012: 178). 
synkrasien anhäufen, vgl. insbesondere die Untersuchung Nüblings $(2000,2011) .{ }^{25} \mathrm{Zu}$ den von ihr beschriebenen Irregularisierungspfaden gehört auch eine stärkere Tendenz zu Assimilationen und Synkopen in den frequentesten Paradigmenzellen. Dies allein berechtigt aber sicherlich nicht zur Annahme einer Kontraktion von andernorts offenbar tolerierten Hiatus.

Somit überzeugt keiner der Versuche, die Singularalternante eines vermeintlich grundsprachlichen Perfekts $* h_{1} e-h_{1} o ́ d-/ * h_{1} e-h_{1} d-$ im Germ. nachzuweisen oder die Übernahme des Pluralvokalismus $\mathrm{zu}$ motivieren. Inwiefern für die Grundsprache überhaupt eine Perfektstammbildung zu dieser Wurzel angenommen werden darf, kann nicht Gegenstand dieses Beitrags sein, ${ }^{26}$ es ist auf jeden Fall festzuhalten, dass in der ital. und germ. Sprachgeschichte im Übergang von einem Aspekt- zu einem Tempussystem zwecks Füllung von paradigmatischen Lücken in großem Umfang Neoperfekta gebildet wurden, nachdem die aspektuell begründeten Beschränkungen gefallen waren (zum Germ. vgl. Kap. 2 inkl. Fn. 9, zum Ital. etwa Meiser 2004: 199). Perfektbelege aus diesen beiden Subphyla haben daher wenig Aussagekraft für die Rekonstruktion der grundsprachlichen Stammbildungen zu einer Wurzel. Wenn sich der Ansatz wie in diesem Fall nur auf das Gr. und Air. stützt und er für das Germ. zu den oben erörterten Problemen führt, ist es sicherlich legitim, alternative Herleitungen von germ. Prät.Sg. * $\bar{e} t$ - zu prüfen, die weniger Zusatzannahmen nötig machen als die oben präsentierten. Einen erfolgsversprechenden Vorschlag hat Bammesberger (1986: 57, 1996: 37) wieder aufgegriffen, der das germanische Präteritum auf ein idg. Imperfekt zurückführt. In diesem Szenario ergibt sich * $\bar{t}$ - im Singular und Plural lautgesetzlich aus einem grundsprachlichen Imperfektparadigma, dessen Entwicklung zum Germ. hin in Tabelle 3 aufgezeigt wird:

\begin{tabular}{|c|c|c|c|}
\hline & indogermanisch & gemeingerm. & gemeingerm., mit Endungsersatz \\
\hline 1. Sg. & $*$ é- $\mathrm{h}_{1}$ ed-m & $>* \bar{e} t u^{n}$ & $\rightarrow * \bar{e}^{\mathrm{a}}$ \\
\hline 2. Sg. & *é-h $h_{1}$ ed-s & $>*_{\overline{e s}}$ & $\rightarrow$ *ēst \\
\hline 3. Sg. & *é-h $\mathrm{h}_{1} \mathrm{ed}-\mathrm{t}$ & $>* \overline{\mathrm{e} s}$ & $\rightarrow * \overline{\mathrm{e}} \mathrm{t}^{\mathrm{e}}$ \\
\hline 1. Pl. & $*$ é-h ${ }_{1}$ d-me & $>* \bar{e}$ tme & $\rightarrow{ }^{*} \operatorname{ètum}^{\mathrm{e}}$ \\
\hline 2. Pl. & $*$ é-h $\mathrm{h}_{1} \mathrm{~d}$-te & $>*^{*} \bar{s} \mathrm{se}$ & $\rightarrow{ }^{*} \bar{e}$ tud ${ }^{\mathrm{e}}$ \\
\hline 3. Pl. & $*$ é-h ${ }_{1} \mathrm{~d}$-ent & $>*$ ètind & $\rightarrow *$ ētun \\
\hline
\end{tabular}

Tabelle 3

Die einzige nötige Zusatzannahme ist der Ersatz der ererbten athematischen Endungen mit den für das germ. Präteritum üblichen $*_{-} a / \neg, *_{-} t *_{-s t},{ }^{27} *_{-} e / \neg, *_{-} u m, *_{-} u d, *_{-} u n$. Abgelehnt wird diese lautlich und formal problemlose Herleitung von * $\bar{e}$ - als Averbo 2 und 3 aufgrund des vermeintlichen Fehlens von Reflexen des Imperfekts im Germanischen sowie fehlender Evidenz für die Verwendung des Augments als Imperfektmarker in ebendiesem Sprachzweig, so etwa von Mailhammer (2007: 79). In der Tat lässt sich die allmähliche Obligatorisierung des Augments in denjenigen Sprachen, die es als Vergangenheitsmarker bewahren, d.h. im Gr., Phrygischen, Armenischen und Indoiranischen, in den Korpora nachvollziehen, sie ist folglich vergleichsweise jung (vgl. Meier-Brügger 2010: 315), doch schließt dies die (fakultative) Verwendung im Spätindogermanischen und Vorgermanischen natürlich nicht aus. Weiter lässt sich die Auffassung, das Imperfekt sei im Vorgermanischen spurlos geschwunden, nicht aufrecht erhalten, zumal zumindest das Präteritalparadigma des westgerm. TUN-Wortes in der Forschung einhellig auf ein idg. Imperfekt zurückgeführt wird, vgl. etwa

\footnotetext{
25 Die Folgen der Hochfrequenz sind teilweise auch synchron sichtbar, vgl. das unterschiedliche flexivische Verhalten von nengl. to behave - she behaves gegenüber hochfrequentem to have - he has.

26 Vgl. verteidigend Schumacher (1998: 185), ablehnend Bammesberger (1986: 57; ${ }^{*} h_{l} e d-$ sei praesens tantum).

27 Vgl. hierzu Fn. 15.
} 
Lühr (1984: 40), Rix \& Kümmel (2001: 137, Fn. 14), Ringe (2006: 158) oder Mottausch (2013: 29-32). ${ }^{28}$ Es stellt sich die Frage, ob man das mögliche Überleben dieses in der germ. Sprachgeschichte ansonsten gänzlich marginalisierten Vergangenheitstempus ${ }^{29}$ im Falle von ESSEN mit hoher Gebrauchsfrequenz in Verbindung bringen darf. Beim TUN-Imperfekt liegt eine solche Erklärung zweifelsohne nahe, zumal das Verb zusammen mit einem Verbalnomen oder Part.Prät. die Präteritalperiphrase ausbildete, die für die Vergangenheitsumschreibung von gemäß communis opinio allen sekundären Präsentien verwendet wurde und aus der im Grammatikalisierungsprozess das gemeingerm. Dentalsuffix entstand. Spätestens durch die Einbettung in diese Periphrase muss TUN in den Höchstfrequenzbereich vorgestoßen sein, wo es in den westgerm. Sprachen bis heute geblieben ist. ${ }^{30}$ Für ESSEN haben wir keine vergleichbaren Hinweise zur Gebrauchsfrequenz. Immerhin sprechen aber die starke Verankerung des mit *et-a- versprachlichten Konzepts in jedem erdenklichen kulturellen Umfeld sowie der Umstand, dass das Germ. nach Ausweis der Korpussprachen kein(e) Synonym(e) zu *et-a-kannte, ${ }^{31}$ dafür, dass dieses zum Hochfrequenzbereich gehörte.

Als Zwischenfazit ist festzuhalten: Die übliche Annahme der Analogiethese, wonach die schwache Alternante eines aus dem Idg. ererbten Perfekts (oder womöglich vor- oder frühgerm. gebildeten Neoperfekts/Präteritums?) des ESSEN-Wortes das Muster für die Dehnstufe in Averbo 3 von Verben der Kl. IV und V geliefert habe, trägt dem Umstand nicht Rechnung, dass sich eine zu diesem Perfekt gehörige starke Alternante nicht nachweisen lässt. Die in der Forschung postulierten Erklärungsversuche für ihr Fehlen überzeugen nicht. Aus altgermanistischer Perspektive ist der Ansatz eines vorgerm. Perfekts zur Wurzel idg. ${ }^{*} h_{1} e d-$ daher durch nichts zu stützen. ${ }^{32}$ Eine Rückführung von Averbo 2 und $3 * \bar{e} t$ - auf ein grundsprachliches Imperfekt ist dagegen lautlich unproblematisch. Spuren grundsprachlicher Imperfekta (Prät. des germ. TUN-Wortes) oder Wurzelaoriste (Dentalsuffix; weiter das germ. WOLLEN-Wort) ${ }^{33}$ zeigen, dass vom idg. Formeninventar mehr übriggeblieben ist (wenngleich auch nur in Relikten), als die meisten Darstellungen der Genese des germ. Verbalsystems mit ihrem Fokus auf Systematisierungs- und Regularisierungstendenzen suggerieren. Wenn das ESSEN-Wort von so hoher Gebrauchsfrequenz war (wie es auch Mottausch 2000: 48-50 annimmt), scheint die Fortsetzung eines idg. Imperfekts durchaus plausibel. Die Bedeutung der Gebrauchsfrequenz des ESSEN-Wortes bei der Ausbreitung des Dehnstufenvokals im Averbo 3 von Kl. IV und V ist Gegenstand des nächsten Kapitels.

28 Mit zusätzlichen Imperfektreflexen im germ. Verbalsystem rechnen Kortlandt (2011) und Mottausch (2013: 15).

29 Für die indikativen Formen des Imperfektparadigmas darf sicherlich ein temporaler Charakter angenommen werden.

30 Vgl. für das Nengl. Leech et al. (2001: 282), für das Nhd. Ruoff (1981: 440). Im Ost- und Nordgerm. wurde das Lexem dagegen scheinbar früh durch got. taujan resp. awn. gera/gøra etc. ersetzt.

31 Die semantische Verengung auf 'fressen' (v.a. von Tieren) und die Verwendung eines anderen Etymons für 'essen' wie im Nisl. (borða 'essen' neben eta 'ds.' (gehoben-veraltend), éta 'essen; fressen'), in gew. nnorw. Varietäten (Bokmål å spise 'essen' neben å ete 'gierig essen; fressen') und im Ndän. (at spise 'essen' neben at cede 'fressen') ist jünger.

32 Womit sein grundsprachliches Alter aber natürlich per se in Abrede gestellt sein soll, womöglich ist es bloß im Laufe der vorgerm. Sprachgeschichte ausgestorben.

33 Zur Beteiligung des Aoristes beim Aufbau des Endungssatzes des germ. schwachen Präteritums vgl. insbesondere Mottausch (2013: 28-35); zum WOLLEN-Wort vgl. Bammesberger (1986: 117f.). 


\section{Versuch einer Rechtfertigung der Analogietheorie: Mottausch (2000)}

Ungeachtet dessen, ob man *ēt- aus einem idg. Perfekt oder Imperfekt gewinnt, basiert die Analogietheorie auf der Grundannahme, dass ein einziges Verb die Basis für die analogische Umgestaltung der Formen von Averbo 3 der restlichen Verben mit Wurzelstruktur $* K_{0} V T$ (sowie sekundär $* K_{0} V R-$ ) stellte. Wie bereits oben erwähnt wird dies als ihre größte Schwäche angesehen. Kortlandt (1992: 103-105) verwirft die Analogietheorie deswegen aber nicht, sondern vermutet, die altgerm. Korpussprachen vermittelten nur ein unvollständiges Bild der ursprünglichen Basis von $*^{-1}$ als Averbo-3-Marker. Ursprünglich hätten im Germ. womöglich mehr vokalisch anlautende Verben existiert, in denen $* \bar{e}^{-1}$ lautgesetzlich habe entstehen können. Dieses Postulat stützt er auf die in der deutschen Sprachgeschichte nachweisbare Tendenz zur Eliminierung vokalisch anlautender Verben. Dies illustriert er unter Verweis auf germ. *aik-a-, *ak-a-, *al-a-, *alb-a-, *an-a-, *ann (Präteritopräsens), *auk-a-, *aus-a- etc., die entweder ausstarben oder nur noch präfigiert fortgesetzt wurden (ahd. an, unnen vs. nhd. gönnen). Diese Tendenz sei also womöglich alt und bereits in germ. Zeit wirksam gewesen, ${ }^{34}$ weswegen andere $*_{e K} \mathrm{~K}$-Verben entweder untergegangen oder infolge Präfigierung nicht mehr direkt als solche erkennbar seien. In der Folge rekonstruiert er für die Verben germ. *nem-a'nehmen', *geb-a- 'geben' und *wes- $a$ - 'sein' für die vor- oder frühgerm. Zeit ganze Paradigmata oder zumindest Perfektformen mit vokalischem Anlaut (resp. mit idg. ${ }^{*} h_{1} e-$ ), in denen im germ. Averbo 3 regelmäßig eine Dehnstufe zu erwarten wäre. Seine Etymologisierungsvorschläge wurden in der Forschung m.W. ausnahmslos abgelehnt, vgl. Mottausch (2000: 47; 55, Anm. 6) und Mailhammer (2007: 80f.). In der Tat sind die von ihm postulierten Metanalysevorgänge, deren Resultat die vermeintlichen germ. Neowurzeln mit konsonantischem Anlaut sind, schwer nachvollziehbar; ferner ergeben sich teilweise auch semantische Probleme (germ. *geb-a- zu idg. * $h_{1} e p$ - 'fassen, ergreifen'?). Zumindest die von ihm postulierte Entstehung des NEHMEN-Wortes erscheint aber nicht völlig abwegig: Hinter germ. *nem-a- verstecke sich ein präfigiertes *gan-em-, dessen Simplex in gleichbedeutend lat. $e m \bar{o}$, lit. dial. emù, air. -eim, -emat vorliege. Die Wurzel wird von Rix \& Kümmel (2001: 236, vgl. ibid. für die einzelsprachlichen Formen) als idg. ${ }^{*} h_{1} \mathrm{em}$ - angesetzt; die gesuchte Dehnstufe wäre folglich aus der schwachen Alternante eines Perfekts idg. $*(\hat{k} o m-) h_{1} e-h_{1} o ́ m-/ * h_{1} e-h_{1} m$ - $^{\prime}$ zu gewinnen. ${ }^{35} \mathrm{Idg}$. *k̂kom- erscheint in den germ. Korpussprachen zwar stets ohne Nasal (z.B. ahd. ga-/gi-), aber dessen gelegentlicher Erhalt als zwischenzeitlicher Hiatustilger nach dem Laryngalschwund resp. nach der Metanalyse von *gan-emzu *ga-nem- als Anlaut einer germ. Neowurzel ist nicht per se implausibel, wenn auch ohne gesicherte Parallelen. Eine echte Verbreiterung der Basis, von der aus die Alternation $* e-* a$ $-*^{-1}$ von K1. IV und V hätte produktiv werden können, ist damit aber natürlich nicht erreicht (immerhin läge aber ein Prototyp für die Adaption von ${ }^{*} \bar{e}^{-1}$ in K1. IV vor).

Das Problem der ursprünglich vermeintlich zu geringen Typenfrequenz des Musters bleibt somit bestehen. Mottausch (2000: 48) entgegnet dieser Kritik, eine solche Basis könne ausreichend sein, sofern 1), ,ein Bedürfnis nach einer Neuerung (z.B. größere Klarheit der neuen Formen)“ bestehe, 2) das Musterverb von hoher Gebrauchsfrequenz sei und 3) „die einfache Handhabbarkeit der Neuerung“" gegeben sei. Dabei verweist er auf acht Beispiele aus

\footnotetext{
34 Kortlandt erklärt aber nicht, weshalb diese Tendenz im Nordgerm. spätestens nach der dialektalen Zergliederung des germ. Kontinuums zu wirken aufhörte, vgl. die Fortsetzer dieser vokalisch anlautenden Verben im Neuskandinavischen wie nschwed. att åka 'fahren, gleiten', nnorw. å ale 'züchten, aufziehen', nisl. auka 'erhöhen', ndän. at øse ‘schöpfen' oder nnorw. å unne 'gönnen'.

$35 \mathrm{Vgl}$. zu idg. *k̂kom- lat. cum, com/con/co-, air. con-, germ. *ga-> nhd. ge-.
} 
der Indogermania, ${ }^{36}$ in denen einst sehr schwach besetzte Flexionsklassen mit hochfrequenten Mitgliedern oder sogar Einzelverben aus dem Hochfrequenzbereich das Vorbild für die analogische Umgestaltung anderer Flexionsklassen abgaben. Zwei seiner Beispiele sollen hier kurz präsentiert und besprochen werden, da sie für die weitere Diskussion wichtige Anhaltspunkte liefern:

1) Das Ahd. und As. zeigen in der 1.Sg.Ind.Präs. der 2. (-ōn-Verben) und 3. K1. (-ên-Verben) der schwachen Verben die Endungen -ōm, -ōn resp. -ēm, -ēn. Mit dieser Endung stehen sie innerhalb der Germania isoliert da, vgl. ahd. salbōm, -ōn 'ich salbe', lëbèm, -ēn 'ich lebe' gegenüber got. salbô, liba, ae. sealfiu, -ie, lifgu, awn. kalla 'ich rufe' (2. K1., das SALBEN-Etymon fehlt im Awn.), lifi. Es wird gemeinhin angenommen, dass $-m$ in den kontinentalgerm. Endungen von K1. 2 und 3 aus dem Personalkennzeichen der 1.Sg. des Präs.Ind. der Athematika übertragen wurde, zu denen nebem dem verbum substantivum (ahd. bim 'ich bin') auch die Verben TUN (ahd. tuam, tuon), GEHEN (ahd. gām, gēm) und STEHEN (ahd. stām, stēm) gehören. Boutkan (1995: 346) sieht diese Übertragung durch das Bedürfnis nach Disambiguierung von Ind. und Opt. motiviert, die sonst homonym wären (wie es im Got. der Fall ist). Wie Mottausch (2000: 49) anmerkt, hat sich im slov. und serbokroat. Verbalsystem die genau gleiche Entwicklung abgespielt, allerdings erfasste der Prozess hier alle Verben.

Die Ausbreitung von $-m$ ausgehend von der mitgliederschwachen Gruppe der Athematika ist ein klassischer Fall eines überstabilen Markers im Sinne Wurzels (1984: 139): Ein Marker einer Paradigmenzelle breitet sich von einer oder mehreren Flexionsklassen auf weitere aus, ohne dass es dabei zu einem Flexionsklassenübertritt kommt. Mit anderen Worten: Es wird nicht gleich das gesamte Flexionsmuster des Geberparadigmas verallgemeinert, die Anzahl Flexionsklassen bleibt also (zumindest vorläufig) unverändert. ${ }^{37}$

2) Wie das Germ. führt auch das Ved. das idg. Perfekt mit Reduplikation sowie Abtönund Schwundstufe im starken resp. schwachen Stamm weiter. Bei einer Handvoll Verben zeigt die schwache Alternante des Perfektstammes aber anstelle des üblichen zu erwartenden schwundstufigen $\mathrm{Ka}_{-} K_{1} K_{2^{-}}$(auch oder ausschließlich) die Form $K_{l} \bar{e} K_{2^{-}}$, vgl. petathuh ${ }^{38}$ 'ihr seid geflogen' neben paptū 'sie sind geflogen' zur Wurzel ved. pat'fliegen'. Dadurch werden unübliche Konsonantencluster vermieden. Diese $\bar{e}$-Formen treten in rgvedischer Zeit erst bei Verben der Struktur KaT- auf, in nachrgvedischer Zeit erfasst das Muster dann immer mehr Verben, auch solche der Struktur KaN-, vgl. Kümmel (2000: 19, 293f.). Mottausch geht - wie seit Bartholomae (1885) üblich davon aus, dass dieser Perfekttyp ursprünglich auf die drei Perfekta ved. sed- (zu sad'sich setzen'), yet- (zu yat- 'eine feste Stellung einnehmen, fest Stellung beziehen') und yem- (zu yam- 'ausstrecken, hinstrecken; halten, zügeln') beschränkt war. In diesen Fällen entstand ved. $\bar{e}$ lautgesetzlich: idg. ${ }^{*} s e-z d->*_{s a-z d->}>$ sed- (regulärer Laut-

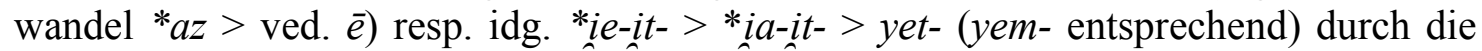
Monophtongierung von $* a i>$ ved. $\bar{e}$. Lubotsky (2012: 177f.) hat aber unlängst

36 Dabei scheint er teilweise auf die Sammlung von Osthoff \& Brugmann (1878: 82f.) zurückzugreifen, die ebenfalls für die Möglichkeit einer Verallgemeinerung von Flexionsmustern ursprünglich tiefer Typenfrequenz argumentieren.

37 Für eine kritische Auseinandersetzung mit diesem Konzept s. Hill (2007: 94-97).

38 Für $\bar{e}$ wird üblicherweise <e> geschrieben; der Vokal wird in der ai. Grammatik zu den Diphthongen gezählt, vgl. Mayrhofer (1978: §5, §14) sowie das Folgende. 
nachgewiesen, dass die Monophthongierung von *ai zur Zeit der Kompilation der Rgveda, in der bereits zahlreiche KaT-Verben im schwachen Perfektstamm nichtlautgesetzliche $e$-haltige Formen zeigten, noch gar nicht erfolgt war. Folglich schmelze die ohnehin schon kleine Gruppe, von der aus sich das $e$-Perfekt ausgebreitet habe, auf ein einziges Vorbild, sed-, zusammen. Sollte Lubotskys Datierung korrekt sein, böte die analogische Ausbreitung von ved. $e$ in der schwachen Perfektstammalternante also ein perfektes Analogon zum in 3. diskutierten Szenario zum Ursprung $\bar{e}$-haltiger Präterita des Germ., ohne dass sich dahinter ältere, gemeingrundsprachliche Zusammenhänge verbergen würden (idg. ${ }^{*} s e-z d$ - konnte im Germ. nur -st- ergeben, vgl. idg. *ni-zd-o > ae., ahd. nest 'Nest').

Mottausch ist mit seiner Annahme, die hohe Tokenfrequenz eines Lexems begünstige die Produktivwerdung seines Flexionsmusters, nicht allein. Auch Kortlandt (1992: 105) und Schumacher (2005: 603), die wie oben dargelegt auf unterschiedlichem Weg zu einer minimalen Basis von vier resp. vier bis sechs Verben gelangen, erklären die Ausbreitung von ${ }^{*} \bar{e}^{-1}$ als Averbo-3-Vokal mit der Hochfrequenz eines „Prototyp[s]“ (Schumacher: ibid.). Auch außerhalb der Altgermanistik ist dieses Argument keineswegs unbekannt. So erachtet Lubotsky (2012: 178) sed-als ausreichende Basis für die Ausbreitung des ved. e-Perfekts, denn, ,[t]he weak forms with $-e$ - are very well attested for this common root $[\ldots]$ and the motivation for introducing this weak grade is evident: most of the clusters *bhj, ${ }^{*} p c,{ }^{*} k$ etc. are phonotactically inadmissible in Sanskrit".

In den allermeisten morphologischen Theorien wird Tokenfrequenz dagegen nicht als Faktor aufgefasst, der die Produktivität eines Musters ermöglicht oder fördert. So kommentiert Bybee (2010: 89): ,type frequency has been found to be an important determinant of productivity, while no such role has been found for token frequency." Der hier manifest werdende Widerspruch zwischen einigen historischen Linguisten und jüngeren morphologischen Theorien ist Gegenstand des nächsten Kapitels. Dabei wird aufgezeigt, dass die Analogiehypothese auch - oder gerade erst recht - im Rahmen jüngerer theoretischer Frameworks eine plausible Erklärung für die Ausbreitung des Präteritalvokals $* e^{-1}$ liefert.

\section{Weil Typenfrequenz nicht alles ist: produktivitätsbestimmende Faktoren und die Ausbreitung von $* \bar{e}^{1}$ in den KI. IV und V}

Produktivität ist bekanntermaßen eines der meistbeachteten Themen der morphologischen Theorie. Befruchtet wurde die Diskussion um ihre Voraussetzungen durch die v.a. in den 1980er- und 1990er-Jahren intensiv geführte Diskussion um eine kognitionswissenschaftlich möglichst plausible Modellierung der Verarbeitung morphologisch komplexer Formen, in der besonders die unterschiedliche Produktion regulärer und irregulärer Flexionsformen im Zentrum stand. Vertreter des Duale-Routen-Modells wie Pinker \& Prince (1992) postulierten, die Produktion regulärer und irregulärer Flexionsformen erfolge über zwei unterschiedliche, streng komplementäre Pfade: Irreguläre Formen seien im mentalen Lexikon als fertige Einheiten repräsentiert und würden als solche abgerufen, reguläre dagegen jedes Mal aufs Neue regelbasiert generiert. Langacker (1987: 42) kritisierte dieses Postulat aus theoretischen Überlegungen, weil nicht auszuschließen sei, dass (hochfrequente) reguläre Formen auch eine eigene Lexikonrepräsentation haben können (,rule/list fallacy“). Den experimentellen Nachweis haben u.a. Baayen et al. (1997) erbracht, die in einem Worterkennungstest mit regulären Flexionsformen frequenzbedingte Unterschiede in der Reaktionszeit der Probanden feststellen 
konnten. Darin sehen sie eine Bestätigung des u.a. in Baayen (1992: 126f.) umrissenen und von Schreuder \& Baayen (1995) genauer definierten dual-route-race-Modells. Dieses geht davon aus, dass im Produktionsprozess sowohl die Regelanwendung (Parsing) wie auch das Abrufen fertiger Formen (direkter Zugriff) parallel erfolgen. Mit zunehmender Tokenfrequenz steige die Wahrscheinlichkeit, dass der direkte Zugriff schneller sei, bei tiefer Tokenfrequenz scheine dagegen der Parsingpfad zum Ziel zu führen, vgl. Baayen et al. (1997: 111f.). Baayen (1992: 140) leitet daraus auch ab, dass die Produktivität einer Regel davon abhänge, ob ihr (auch) Lexeme mit tiefer Tokenfrequenz folgen würden, zumal es nur bei der Produktion dieser Formen zu einer Regelanwendung kommt. ${ }^{39}$ Einen radikaleren Standpunkt nimmt Bybee $(1985,1988,1995)$ ein, die für eine gänzliche Aufgabe einer Dichotomie ,morphologische Regeln“ vs. „lexikalische Repräsentation“ plädiert. Sie beschreibt das mentale Lexikon stattdessen als ein Netzwerk von gespeicherten items, denen je nach Wortfrequenz eine unterschiedlich hohe lexikalische Stärke zukommt. Hohe lexikalische Stärke bedeutet dabei relative Autonomie von anderen Lexemen. Mit abnehmender lexikalischer Stärke werden items dafür stärker mit anderen verknüpft (sog. Verbindungen), was durch semantische und phonologische Übereinstimmungen erfolgt. Die Verbindungen können dabei mal loser, mal enger ausfallen, entsprechend variiert auch die sog. Verbindungsstärke. Solche miteinander verbundenen items bilden nun Schemata aus. So tragen engl. Gerundive auf -ing durch ihre funktional-phonologische Übereinstimmung ein Schema der betreffenden Kategorie. Eine von diesen items unabhängige Regel gebe es nicht, vielmehr werde bei der Produktion morphologisch komplexer Formen versucht, ein item einem Schema anzuschließen. Solche Schemata könnten wie im Falle der engl. Gerundive sehr unspezifisch und offen sein und items jeder phonologischen Struktur attrahieren. In anderen Fällen ergäben sich Verbindungen hingegen nur bei einer Kombination aus semantisch-funktionaler Identität und hoher Übereinstimmung phonologischer Merkmale zwischen den items. So konstituieren im Engl. Verben mit Präterita der phonologischen Gestalt [C (C) (C) $\Lambda$ Nasal/Velar $]_{\text {past }}$ (vgl. string - strung) ein relativ starkes Schema, dem sich weitere Verben mit passendem Konsonantengerippe anschließen können (z.B. in verschiedenen Nonstandardvarietäten bring - brung). Die Formulierung einer Regel, der zufolge man ähnlich wie im System der starken Verben im Germ. Verben einer bestimmten Wurzelstruktur automatisch einen gewissen Präteritalvokal zuweist, funktioniert hier schon allein aufgrund des sehr unterschiedlichen Präsensvokalismus der engl. Verben nicht, das Schema muss sich also über die Vergangenheitsformen konstituiert haben (in der Bybee'schen Terminologie handelt es sich daher um ein sog. Produktorientiertes Schema). Verben mit der Wurzelstruktur [s (C) (C) V y] sind in der Klasse besonders zahlreich, wodurch sie womöglich einen Prototyp darstellen. Für einen Übertritt in diese Klasse sei eine strikte Übereinstimmung im Konsonantismus nicht zwingend erforderlich, denn auch Verben mit wurzelschließendem Velar wie dig oder strike haben sich dem Schema angeschlossen. Bybee geht daher davon aus, dass der Anschluss an das Schema über die Assoziierung nach dem Konzept der Wittgenstein'schen Familienähnlichkeit erfolgen kann: Einige Mitglieder der Klasse wie string sind prototypische, zentrale Vertreter; andere, peripherere Mitglieder zeigen dagegen nur eine gewisse Ähnlichkeit zu diesem Prototyp. Bauer (1997: 554) zieht daraus die Schlussfolgerung, solche periphereren Mitglieder könnten, weil die Verbindungen zum Klassenschema schwächer sind, auch von anderen Schemata angezogen werden, womit die Variation in der Vergangenheitsbildung verschiedener nengl. Verben relativ einfach verständlich wird. Ausgehend von diesen Prämissen ergibt sich im Bybee'schen Netzwerkmodell, dass der Produktivitätsgrad eines Schemas zum einen von seiner Allgemeinheit resp.

39 Interessanterweise ist einer der Verfechter des Duale-Routen-Modells, Steven Pinker, später von der Position einer strengen rule-list-Dichotomie abgerückt, vgl. Pinker (1999: 137). 
Spezifizität hinsichtlich der erforderlichen phonologischen Übereinstimmung zwischen den Mitgliedern abhängt. Zum anderen werde Produktivität von Typenfrequenz beeinflusst, denn durch eine hohe Anzahl an Mitgliedern werde die Verbindungsstärke und somit die Attraktionskraft des Schemas erhöht. Im Umkehrschluss bedeutet dies auch, dass ,,[a] verb of high token frequency will not serve to strengthen a schema; in fact, it appears that very highfrequency verbs have very little effect on productivity, since [...] such forms seem to be processed without forming connections with other items" (Bybee 1988: 138). Im Extremfall kann hohe lexikalische Stärke zu einem so hohen Grad an Entrenchment führen, dass sich das Lexem von seinem flexivischen Muster gänzlich emanzipiert. Die Verwendung des Lexems führt folglich nicht mehr zur Mitaktivierung der zugrundeliegenden schematischen Struktur, womit seine hohe Tokenfrequenz der Produktivität sogar abträglich sein kann, vgl. Barðdal (2008: 49).

Zwei zentrale Behauptungen des Netzwerkmodells müssen uns also beschäftigen: Zum einen die Auffassung, Produktivität sei maßgeblich von Typenfrequenz abhängig und zum anderen die Behauptung, eine hohe Tokenfrequenz wirke nicht produktivitätssteigernd oder im Extremfall sogar produktivitätsmindernd. Wenden wir uns der ersten Behauptung zu. Die Auffassung, nur Flexionsmuster hoher Typenfrequenz könnten neue Lexeme attrahieren, findet sich auch in anderen theoretischen Frameworks wieder. So wird Typenfrequenz auch in den älteren Konfigurationen der Natürlichen Morphologie (Wurzel 1984: 96f., 127, 129-131, 168f., relativierend 154f.) oder dem theorieneutralen Beitrag Haspelmaths (2006: 60) als entscheidender Faktor für Produktivität beschrieben. Diese Sichtweise wurde jedoch verschiedenenorts als zu einseitig kritisiert, vgl. u.a. die Diskussion bei Enger (2010: 85-89). So bemerkt Bauer (2001: 62), nachweislich produktive Regeln wie der nhd. Default-Plural auf $-s$ wiesen eine tiefe Typenfrequenz auf. Eine hohe Typenfrequenz sei zudem ,the result of past productivity rather than an indication of present productivity" (Bauer 2001: 48f.). Argumente gegen eine zu einseitige Betonung des Faktors Typenfrequenz liefert auch die Sprachgeschichte, lässt sich doch in Sprachen mit weit zurückreichender Überlieferung die Ausbreitung von Regeln mit ursprünglich tiefer Typenfrequenz zweifelsfrei nachweisen, vgl. z.B. das in diesem Kontext oft genannte Beispiel der nhd. Pluralmarkierung mittels Umlaut und -er, die von einer Handvoll althochdeutscher Neutra des Typs lamb - lembir ausging. Vergleichbare Beispiele sind auch aus der Wortbildung bekannt, vgl. Fertig (2013: 113). Somit scheint die Kritik an der Analogietheorie eher auf ein Scheinproblem denn ein reales Bezug zu nehmen. Wie aber ist Mottauschs Argument zu beurteilen, der Siegeszug von $* \bar{e}^{l}$ sei mit der hohen Tokenfrequenz des Vorbildes *et- $a$ - zu erklären? Aus Bybees Postulat darf sicherlich nicht der Schluss gezogen werden, Flexionsmuster oder die Marker einzelner Paradigmenzellen hochfrequenter Lexeme könnten sich unter keinen Umständen analogisch auf weitere Lexeme ausbreiten. So beschreibt Nübling (2001: 57f.; 2011: 147) beispielsweise, wie die hochfrequenten athematischen Verben TUN, SEIN, STEHEN und GEHEN des Westfries. nicht nur unter Verallgemeinerung des Präteritalvokalismus -ie- ihre Präterita aneinander angeglichen haben (dwaan - die/diene(n), wêze - wie/wiene(n), stean und gean entsprechend; -ie- mit unbekannter Herkunft), sondern auch das hochfrequente und einst regulär nach der 3. Kl. der schwachen Verben flektierende $h a$ 'haben' in ihre Gang attrahiert haben. ${ }^{40}$ Ähnliche Prozesse von „(Kleinst-)Gruppenbildungen“, die zu ,relative[r] (interparadigmatische[r]) Regularität" führen (Nübling 2000: 268, zum Westfries.), sind auch aus dem Aspan.

40 Vergleichbare Angleichungen haben sich im Westfries. im Paradigma der starken Verben jaan 'geben' und slaan 'schlagen' (unter zusätzlichem Einfluss der Athematika/Kurzverben stean und gean) sowie in der Präsensflexion von dwaan, sjen 'sehen' und tsjen 'ziehen' abgespielt, vgl. Nübling (2000: 112-115, 307; 2011: 147f.). 
bekannt, vgl. die bei Lloyd (1987: 304) beschriebene Herausbildung einer Gruppe von Verben mit Perfektausgang -ove (aspan. andar 'gehen' - andove, seer 'sitzen' - sove, creer 'glauben' - crove, crescere 'wachsen' - crove (sic!), atrever 'wagen' - atrevo und weitere) nach dem Vorbild von tener 'halten; haben'- tove. Häufig erfolgen solche „local optimizations“ (Gaeta 2006: 20) auf Basis außermorphologischer Eigenschaften (im Sinne der Wurzel'schen systembezogenen Natürlichkeit, vgl. u.a. Wurzel 1984: 117f., s. dazu auch unten), wie im Falle der Klasse der aspan. Verben mit -ove-Perfekta, der sich v.a. Verben mit einsilbiger Wurzel anschlossen. In anderen Fällen wie den westfries. -ie-Präterita konnte die lokale Analogie aber scheinbar auch ohne solche „Brücken“ erfolgen, zumindest, wenn die betroffenen Verben hochfrequent sind, vgl. Nübling (2011: 147). Die westfries. und aspan. Beispiele zeigen, dass auch ausschließlich durch Hochfrequenzverben (also „lexikalisch starke“ Verben) instanziierte Schemata produktiv werden können, doch scheinen nur andere eher frequente Verben Verbindungen zu diesem Schema aufzubauen, vgl. Nübling (2000: 269). Durch die von Bybee angenommene Gradualität der Zugehörigkeit von Verben zu Schemata - es gibt prototypischere und peripherere Mitglieder, und letztere können auch mit anderen Schemata Verbindungen ausbilden - umschifft das Netzwerkmodell allerdings die Frage, inwieweit Analogien, die durch die lexikalische Stärke eines Verbs möglich wurden, auch nicht-hochfrequente Verben erfassen können. Das Postulat einer sehr hohen Verbindungsstärke von niederfrequenten items legt zwar die Schlussfolgerung nahe, dass dies relativ unwahrscheinlich ist, aber explizite Aussagen dazu fehlen. Entsprechend fragt Bauer (1997: 554) zu Recht: ,,[H]ow does the native speaker (or, a fortiori, the linguist) know what the relevant phonological class of verbs is?" Die Betonung der Gradualität von semantischphonologischen Übereinstimmungen und die Möglichkeit, dass ein Lexem an unterschiedlichen Schemata andocken kann, scheint fast einem flexionsmorphologischen anything goes gleichzukommen. Dabei ist aber zu bedenken, dass die Schlussfolgerungen weitgehend auf Basis der engl. Verbalmorphologie gezogen wurden. Elizitationstests zur schwed. Nominalmorphologie (Linell 1977) und dt. Verbalmorphologie (Penke 2006: 68) scheinen ein anderes Bild zu zeichnen. In diesen Tests wurde untersucht, wodurch die Zuweisung von NonceWörtern zu konkurrierenden Klassen (oder Schemata) gesteuert wird, wobei eine voll produktiv, die andere nur teilproduktiv war. In beiden Fällen war die Zuweisung zur teil- oder nichtproduktiven Klasse nur dann möglich, wenn der Klasse Lexeme angehören, die in der Grundform $^{41}$ mit den Nonce-Wörtern reimen. Wurzel (1984: 136), der auf die schwed. Fallstudie sowie nur durch Reim ermöglichte Flexionsklassenübertritte im Awn. hinweist, hält diesen Fall von Flexionsklassenzuweisung für ,einen extremen Fall der Nutzbarmachung phonologischer Eigenschaften für die Prädizierung der morphologischen Eigenschaften der Wörter, also ihrer Flexionsklasse.“ Dadurch können auch eigentliche Klassen in Form „sehr kleine[r] Teilklassen“ entstehen (ibid.). Diese Bedeutung von Reimwörtern bei Flexionsklassenübertritten oder der Ausbreitung überstabiler Marker lässt eine Ausbreitung des Musters *et-a- - *ettum auf die Reimwörter *met-a- 'messen', *get-a- 'bekommen' und *fet- $a$ - 'fallen', auf deren Bedeutung auch Mottausch (2000: 53) und Mailhammer (2007: 84, Fn. 101) hinweisen, äußerst wahrscheinlich erscheinen. Damit ist die Ausweitung der Analogiebasis ohne weiteres erklärbar. ${ }^{42}$

41 Beim nschwed. Substantiv die Form des unbestimmten Singulars, beim nhd. Verb der Infinitiv.

42 In Penkes (2006) Elizitationstest zur Bildung neuhochdeutscher Partizipia zeigte sich, dass keinesfalls zu allen mit starken Verben reimenden Nonce-Verben starke Partizipialformen gebildet werden, d.h. die Verben konnten auch vom generelleren Schema resp. der voll produktiven Klasse angezogen werden. Bei Formen von Averbo $3 \mathrm{im}$ Germ. war ein Übertritt in eine der schwachen Klassen allerdings keine Option, da sich die Zugehörigkeit eines Verbs zu den starken Verben der Klassen I-V (und die Abgrenzung zu den Klassen der 
Wenn wir den begünstigenden Einfluss von Reimwörtern auf die Etablierung von Flexionsklassen anerkennen möchten, müssen wir uns aber fragen, wodurch die weitere Ausbreitung von $* e^{-1}$ als Marker von Averbo 3 auf nicht mit *et-a-reimende Verben ermöglicht wurde, wie *les- $a$ - 'sammeln', *geb- $a$ - 'geben' in K1. V und * $k^{w} e m-a-\sim * k u m-a$ - 'kommen', * dwel-a- 'verharren' etc. in Kl. IV. Die im Rahmen der Analogietheorie postulierte Produktivwerdung des überstabilen Markers $* \bar{e}^{-1}$ scheint m.E. nur durch die Berücksichtigung der systembezogenen Natürlichkeit im Sinne Wurzels (1984: 75-109) verständlich. Ausgehend von der Beobachtung, dass der Sprachwandel universellen Natürlichkeitsprinzipen wie konstruktionellem Ikonismus, Uniformität und Transparenz zuwiderlaufen kann, postuliert Wurzel, dass die Natürlichkeit eines flexionsmorphologischen Prozesses nur vor dem Hintergrund des einzelsprachlichen Flexionssystems und dessen sog. systemdefinierender Struktureigenschaften eruiert werden könne. Der Beurteilung, ob flexionsmorphologischer Wandel „natürlich“ oder „markiert“ ist, muss also eine Beschreibung des einzelsprachlichen Flexions- oder Teilflexionssystems vorausgehen. Dieses werde durch die folgenden „Hauptparameter" charakterisiert (Wurzel 1984: 82):

a) ein Inventar an Kategoriengefügen und ihnen zugeordnete Kategorien;

b) das Auftreten von Grundformflexion bzw. Stammflexion;

c) die separate vs. kombinierte Symbolisierung von Kategorien unterschiedlicher Kategoriengefüge;

d) die Anzahl und Ausprägung der formalen Distinktionen unter den Flexionsformen eines Paradigmas;

e) die auftretenden Markertypen bezogen auf die beteiligten Kategoriengefüge;

f) das Vorhandensein vs. Nichtvorhandensein von Flexionsklassen.

Für unsere Thematik reicht es aus, wenn wir uns auf den Parameter (e) konzentrieren, denn der angenommene Wandel wie *ge-gநum $\rightarrow{ }^{*}$ gēbum nach dem Vorbild von *ētum führt nur hier $\mathrm{zu}$ Veränderungen, vgl. 2. Zu den Markertypen ist festzuhalten, dass im System der starken Verben ${ }^{43}$ nach der Aufgabe der Reduplikation die Kategorie Tempus (und im Präteritum auch Numerus, zumindest hinsichtlich der Opposition Sg. vs. Pl.) im überwiegenden Fall durch die Markertypen Ablaut und unterschiedliche Endungssätze symbolisiert wurde. Ein die Averbos 1 bis 3 eindeutig disambiguierender Ablautvokal ist also bei $e$-Verben ein systembezogen natürlicher Marker, wobei sich seine Natürlichkeit (oder „Normalität" $)$ in diesem Fall aus der Typenfrequenz ergibt (K1. I-IV gegenüber K1. V). ${ }^{44}$ Die

schwachen Verben) im Vergleich zum Nhd. in viel mehr Paradigmenzellen manifestierte (Infinitivendung *-an-, Vokalalternation zwischen den Averbos 1, 2 und 3, anfänglich Reduplikation, eigener Endungssatz). Diese ,starken“ Paradigmenzellen verlangten ein dem Typ der starken Verben entsprechendes flexivisches Verhalten auch in den zu Averbo 3 gehörigen Zellen. Vgl. zur Bedeutung implikativer Paradigmenstrukturbedingungen zuletzt Dammel (2011: 20-22, mit Lit.).

43 Die Beschränkung auf die starken Verben ist zulässig, vgl. die vorausgehende Fußnote.

44 Haspelmath (2006: 60) argumentiert, das Markiertheitskonzept (das der Natürlichkeit ,umgekehrt proportional [...] zugeordnet“ ist, Wurzel 1984: 21) der Natürlichen Morphologie sei „superfluous“, weil die Entscheidung, welche Ausdrucksverfahren und Marker natürlich seien und demnach produktiv werden können, eine rein quantitative sei, also vom Faktor Typenfrequenz abhängig sei. Ein solcher Monokausalismus kann angesichts gesicherter Fälle, in denen Marker mit ursprünglich tiefer Typenfrequenz produktiv wurden, aber keinesfalls vertreten werden. Auch wenn Wurzel selbst Typenfrequenz für einen wichtigen Faktor für die Bestimmung von Markiertheit/Natürlichkeit hält (vgl. oben), trägt sein Konzept der systembezogenen Natürlichkeit mit den oben zitierten Parametern dem Umstand Rechnung, dass in gewissen Fällen von Sprachwandel Natürlichkeit nicht nur „durch die Ausbreitung einer konkreten Flexionsklasse, sondern durch die Ausbreitung allgemeinerer Strukturprinzipen im Flexionssystem“ (Wurzel 1984: 79) 
Verallgemeinerung von $* \bar{e}^{-1}$ in K1. V nach dem Vorbild des reduplikationslosen Averbos 3 *ētum (*mētum etc.) bringt nun die *KeT-Verben in Übereinstimmung mit den übrigen Klassen, in denen die Markierung einer Opposition Averbo 1 vs. Averbo 2 vs. Averbo 3 ohne Reduplikation möglich war. Weiter kann man vermuten, dass im Averbo 2 von *KeT-Verben die Reduplikation gleich früh beseitigt wurde wie in den Kl. I-IV (*fat wie *raid, *tauh, * malk und *bar) und die Tempuskategorie innerhalb des Paradigmas eines *KeT-Verbes im Singular durch zweifache, im Plural dagegen durch dreifache Exponenz charakterisiert war. Die Analogie führte also zu intraparadigmatischer Einheitlichkeit hinsichtlich der am Tempusausdruck beteiligten Markertypen. Dieses Räsonnement kann indes für die Aufgabe der phonotaktisch unproblematischen Schwundstufe in Kl. IV (*numum $\rightarrow{ }^{*}$ nèmum), die eine systembezogen absolut natürliche Tempusmarkierung ermöglichte, nicht geltend gemacht werden. Matzel (1970: 178) vermutet, die Ersetzung des Schwundstufenvokals durch die Dehnstufe habe ihren Ausgangspunkt in Verben mit schwundstufigem Präsensvokalismus gehabt (diese werden in der Altgermanistik meist „Aoristpräsentien“ genannt, vgl. für das Detail und den ungeklärten Status ihres Alters Bammesberger 1984, insbesondere 22). Als Beispiele nennt er aus K1. IV die Verben germ. *kum- $a$ - 'kommen', *wul-a- 'wallen', aus K1. $\mathrm{V} *$ sub- $a$ - 'schlafen', *trud- $a$ - 'treten' und *knud- $a$ - 'kneten'. ${ }^{45}$ Averbo 1 und 3 hätten in diesen Verben denselben Stammvokal gezeigt, und dieser Missstand sei durch die Übernahme der Dehnstufe beseitigt worden. Diese Argumentation überzeugt auf den ersten Blick nur bedingt, da Übereinstimmungen im Stammvokalismus zwischen Averbo 1 und 2 offenbar teilweise geduldet wurden, vgl. awn., aschwed. koma - kom und aschwed. sova - sof, vgl. Noreen (1904: §536). Dies ist umso erstaunlicher, als das Idg. ein Perfekt zu den Wurzeln * gem- '(wohin) gehen, kommen' und *suep- 'einschlafen' kannte (vgl. Rix \& Kümmel 2001: 209, 612) und got. qam, ahd., as. quam resp. ae. swaef, awn. svaf ein abtönstufiges Averbo 2 für das Germ. auch indizieren. Die nordgerm. Averbo-2-Formen mit Stammsilbenvokal -o- könnten - sofern sie wirklich idg. ${ }^{*}-g^{u} m$ - resp. ${ }^{*}$-sup- fortsetzen - Reflexe eines alten Aoristes sein, ${ }^{46}$ wobei dann aber die Senkung $* u>o$ zu erklären wäre. ${ }^{47} \mathrm{Ihr}$ Überleben in einer direkten Konkurrenzsituation mit dem systembezogen natürlichen Präteritum $\left(* k^{w} e-k^{w} a m-\right)$ wäre vor dem Hintergrund der Tendenz zur Bevorzugung kürzerer Formen im Höchstfrequenzbereich zu verstehen, die, wie Nübling (2011: 145) festhält, sich nicht nur zwischen verschieden frequenten Lexemen, sondern auch zwischen unterschiedlichen Kategorienwerten bemerkbar macht: „the present is always more strongly affected than the past tense. The same holds for the singular vs. plural." Nübling bezieht sich hierbei zwar auf durch Assimilationen entstandene Kürzungen, aber die Tendenz kann sich auch bei der Integration verschiedener Stammbildungen in ein Paradigma geltend gemacht haben, wie in jüngerer Zeit das Beispiel des nhd. HABEN-Paradigmas zeigt, dessen Formen sich aus mhd. haben und dem Kurzverb hân speisen, vgl. dazu Nübling (2000: 23). Wenn wir Matzels Annahme also teilen, dass die Formendisambiguierung in ${ }^{*} K u R$-Wurzeln zur Übernahme des Stammsilbenvokals ${ }^{*} \bar{e}^{-}$führte, fand in der Terminologie der Natürlichen Morphologie in diesen Fällen ein für die

erreicht wird. Hier rücken Parameter (d) und (e) in den Blick: Tempus und Numerus (im Prät.) werden im System der $e$-Verben durch eigene Ablautvokale mitmarkiert. Dieses „Strukturprinzip“ förderte die Ausbreitung des überstabilen Markers $*^{-1}$ trotz seines anfänglich stark beschränkten Vorkommens.

45 Die genaue Herleitung der germ. „Aoristpräsentien“ ist umstritten (zumindest *wul-a- könnte als Fortsetzer eines akrostatischen Präsens gelten, sofern man diesen Stammbildungstyp ansetzen möchte, vgl. Kümmel 2004). Diese schwundstufigen Verben haben häufig normalstufige Doubletten neben sich, vgl. * $k^{w} e m-a$-, *swef-a-, *tred-a- und *kned-a-.

46 So etwa Ljungstedt (1887: 112f.).

47 Sekundärer Antritt der frühgerm. Endung der 1.Sg.Prät.Ind. *-a und späterer analogischer Ausgleich der 2. und 3. Pers.? 
Tempuskategorie der starken Verben natürlicher Markertyp weitere Verbreitung. Weiter wird der Distinktionstyp Averbo $1 \neq$ Averbo $2 \neq$ Averbo 3 (mit Ablaut als Ko-Marker), der innerhalb der Kl. I-V üblich ist, verallgemeinert (vgl. dazu den Wurzel'schen Hauptparameter (d) oben). Matzel erläutert nicht, aus welchem Grund ein Verb von Kl. IV mit der Wurzelstruktur *KeR-, beispielsweise ein ursprüngliches *dwel-a- 'verharren' - *dwal - *dwulum, ebenfalls zu Averbo $3 * d w e \overline{l u m}$ neuerte. Auch die Natürliche Morphologie bietet auf den ersten Blick keine Antwort $-{ }^{*} d w u l u m$ und $* d w e \overline{e l u m}$ sind systembezogen beide gleichermaßen natürlich. Es ist aber zu beachten, dass - wie bereits in einem anderen Kontext erwähnt - in der Natürlichkeitstheorie die Koppelung von Flexionsklassenzugehörigkeit an außermorphologische Eigenschaften als zentraler Mechanismus beschrieben wird, mit dem Sprecher die Prädizierbarkeit der Flexionsklassenzugehörigkeit von Lexemen bewahren. Dies hat sich sicherlich auch bei der Bildung der verschiedenen Averbo-Formen starker Verben mit einmorigem Wurzelsilbengipfel $\left({ }^{*} e\right.$ oder $* u$, d.h. Kl. III-V) geltend gemacht, war doch deren Gestalt vor dem Reduplikationsverlust synchron noch von der Wurzelstruktur ableitbar, also anhand außermorphologischer Eigenschaften prädizierbar. Die Ausbreitung von $* e^{-1}$ in K1. V dürfte diese Prädizierbarkeit vorerst noch nicht direkt bedroht haben: Folgte auf den Wurzelsilbengipfel ein Obstruent als Wurzelsilbenauslaut, galt (neu) die Alternation ${ }^{*} e{ }^{*} u-{ }^{*} a-{ }^{*} \bar{e}^{l}(\mathrm{~K} 1 . \mathrm{V})$. Folgte ihm dagegen ein Resonant (Kl. IV) oder ein Resonant-Obstruent-Cluster (Kl. III), hatte die hier ursprüngliche Alternation ${ }^{*} e / u-{ }^{*} a-{ }^{*} u$ vorerst weiterhin Bestand. Als nun aber die Verben der Struktur *KuR-wie *kum- $a$ - oder *wul-a-zwecks Disambiguierung von Averbo 1 und 3 den überstabilen Marker $* \bar{e}^{-1}$ übernahmen, verlor die Wurzelstruktur ihren Status als flexionsklassenindizierendes Merkmal. Nachdem die Lautklasse der im Wurzelsilbenauslaut stehenden Konsonanten keine sichere Flexionsklassenzuordnung mehr erlaubte, trat ein anderes außermorphologisches Merkmal an ihre Stelle, nämlich dasjenige der Silbenlänge: Verben mit kurzer Wurzelsilbe $\left(*_{-}-e / u R\right.$ - resp. ${ }^{*}-e / u T$-) erhalten im Averbo 3 den Vokal $*^{-1}$, solche mit langer Wurzelsilbe $(*-e R K-) * u$. Die „Umkoppelung“ der Flexionsklassenzugehörigkeit von der Wurzelstruktur an die Silbenlänge der Wurzel dürfte ein langwieriger, sukzessiver Prozess gewesen sein, der die allmähliche Ausbreitung von $* \bar{e}^{l}$ auf die - ohnehin nicht sehr zahlreichen $-{ }^{*}-e / u R$-Verben zur Folge hatte.

\section{Zusammenfassung}

Ziel dieses Beitrages war, die Vorzüge der Analogiehypothese für die Erklärung von Ursprung und Ausbreitung des überstabilen Markers ${ }^{*} \bar{e}^{-}$in den Kl. IV und V aufzuzeigen, nicht zuletzt auch in Gegenüberstellung zu anderen Lösungsansätzen. Als einziges gesichertes Musterverb wurde in Übereinstimmung mit bisherigen Forschungsbeiträgen *et- $a$ - mit seinen Präteritalaverbos $* \bar{e} t-{ }^{*} \bar{e} t u m$ identifiziert. Die in jüngeren Beiträgen einhellig vertretene Annahme, es handle sich dabei um die Kontinuanten eines idg. Perfekts, wurde hingegen verworfen, weil dann in Averbo $2 \dagger(e-) a t$ anstatt des von den Einzelsprachen indizierten * $\bar{e} t$ zu erwarten wäre. Die von der Forschung bemühten Argumente für einen vermeintlichen (lautgesetzlichen oder analogischen) Wandel von *(e-)at $\mathrm{zu} * \bar{e} t$ würden Zusatzannahmen nötig machen, die entweder unplausibel sind oder im direkten Widerspruch zur germ. Lautgeschichte stünden. Aus diesem Grund wurde mit Bammesberger (1986, 1996) die Rückprojizierung auf ein altes augmentiertes Imperfekt erwogen, das (unter der naheliegenden Annahme eines späteren Endungsersatzes) direkt zu den Präteritalaverbos *ēt $-* \bar{e} t u m$ führt. Die in der Altgermanistik gängige Annahme, aus dem idg. Formensystem hätten nur einige Präsensstammbildungstypen und das Perfekt bis ins Germ. überlebt, greift angesichts klarer 
Aorist- und Imperfektspuren (besonders in hochfrequenten Verben) offenbar zu kurz. Die Weiterführung eines Imperfekts zum sicherlich gebrauchsfrequenten ESSEN-Wort ist daher nicht unplausibel. Im zweiten Teil des Beitrages wurde dargelegt, wie die Altgermanistik die Ausbreitung des überstabilen Markers $* e^{l}$ auf Verben der Wurzelstruktur ${ }^{*} K_{0} e T$ - und (sekundär) ${ }^{*} K_{0} e R$ - ausgehend von nur einem Verb zu erklären versucht hat. Die Betonung der Gebrauchsfrequenz als produktivitätsbestimmender Faktor vonseiten der Altgermanistik steht der Annahme in jüngeren morphologischen Theorien gegenüber, dass Typen-, nicht Tokenfrequenz über die Produktivwerdung eines Musters entscheide und die hohe Tokenfrequenz eines Musterverbs der Produktivität sogar abträglich sein könne. Die Identifizierung einer hohen Typenfrequenz als eine Voraussetzung von Produktivität ist in Theorien wie der Natürlichen Morphologie oder dem Netzwerkmodell psycholinguistisch und auch hinsichtlich der diachronen Evidenz gut abgestützt. Das bedeutet aber keineswegs, dass die Analogiehypothese verworfen werden müsste. Im Gegenteil wird die Übertragung von $* e^{-1}$ auch ausgehend von einer kleinen Basis verständlich, wenn das Konzept der systembezogenen Natürlichkeit der Natürlichen Morphologie (Wurzel 1984) in die Diskussion miteinbezogen wird. Der Präteritalvokal $*^{-1}$ war ein innerhalb der starken Verben systemangemessener (natürlicher/normaler) Marker und ermöglichte die Ko-Markierung der hochrelevanten Tempuskategorie auch ohne Reduplikation, die bei starken Verben mit Wurzelvokal ${ }^{*} e$ im Abbau befindlich war und damit zunehmend zu einem gemäß der Natürlichkeitstheorie markierten (d.h. nicht-normalen) Markertyp wurde. Die Ausbreitung wurde zudem durch die Tendenz der Koppelung von phonologischer Struktur und Flexionsklassenzugehörigkeit (Koppelung an ,außermorphologische Eigenschaften“ gemäß Wurzel 1984) gefördert. Die Ausbreitung in den Kl. IV und V lässt sich in der folgenden Szenariokette wie folgt einordnen:

1) Im germ. Präteritum wird die Reduplikation in starken Verben mit Wurzelvokal * $e$ resp. Wurzelreim * $a K$ - zusehends abgebaut (Aoristeinfluss? Abbau multipler Exponenz?).

2) Die Alternation zwischen Averbo 1 und 3 im Verb $* e t-a-,{ }^{*} e-*^{*} \bar{e}^{l}$, wird auf Reimverben wie *met-a-oder *fet-a- übertragen.

3) Alle Verben der Wurzelstruktur $* K_{0} V T$ - übernehmen den überstabilen Marker $* \bar{e}^{l}$, der systembezogen natürlich/angemessen ist.

4) In Verben von Kl. IV $\left(* K_{0} V R-\right)$, wo im Allgemeinen die lautgesetzliche Alternation $* e-$ $* a-{ }^{*} u$ herrscht, wird $*^{-1}$ von Verben mit Wurzelvokal $* u$ übernommen, da in diesen Fällen zwischen Averbo 1 und 3 die Kategorie Tempus nur durch die Ausgänge (und ggf. noch durch Reduplikation), nicht aber durch das systemangemessene Ausdrucksverfahren der Stammmodulation markiert wurde. Als Muster für die Analogie dienten Verben wie *sub- $a$ - mit Averbo 3 *sēbum von K1. V.

5) Durch die Ausbreitung von $* \bar{e}^{-1}$ auf einige Mitglieder von Kl. IV kann die Bildung des Stammes von Averbo 3 nicht mehr anhand der außermorphologischen Eigenschaft Wurzelstruktur $\left({ }^{*} K_{0} V T\right.$ - vs. $\left.{ }^{*} K_{0} V R-\right)$ prädiziert werden. Das Vorkommen von ${ }^{*} \bar{e}^{-1}$ (gegenüber $* u$ ) bei starken Verben ohne Diphthong in der Wurzel wird sekundär an die Silbenlänge gekoppelt: Verben mit kurzer Wurzelsilbe $\left({ }^{*} K_{0} e / u K-\right)$ zeigen im Averbo 3 ${ }^{*} \bar{e}^{1}$, solche mit langer Silbe $\left({ }^{*} K_{0} e R K-\right) * u$. In der Folge übernehmen die wenigen verbleibenden ${ }^{*} K_{0} e R$-Verben mit lautgesetzlichem $* u$ in Averbo 3 den neuen Prät.Pl.Stammvokal $* \bar{e}^{-1}$.

Natürlich hat die hier skizzierte Ausbreitung einen stark hypothetischen Charakter. Das vorgelegte Szenario zeigt aber m.E., dass für die Erklärung des Auftretens von $* e^{-1}$ im germ. starken Verb plausible Annahmen zur Produktivwerdung ursprünglich typenschwacher Muster 
ausreichen. Wir sind aus altgermanistischer Warte also mitnichten genötigt, bei der Bildung schwacher Perfektalternanten im Idg. von vermeintlichen (und widerlegbaren) phonotaktisch bedingten Regeln auszugehen oder bei der Genese des germ. Präteritums den Einfluss dehnstufiger Aoristbildungen anzunehmen.

\section{Dank}

Mein Dank gebührt Pino Marco Pizzo (Johannes Gutenberg-Universität Mainz), Hans-Olav Enger (Universitetet i Oslo), Stephen Anderson (Yale University), Sandro Bachmann, Michael Erlach, Francesco Gardani und Anja Hasse (alle Universität Zürich) für wertvolle Diskussionen und Anregungen.

$\begin{array}{llll}\text { Abkürzungen } & & \\ \text { ahd. } & \text { althochdeutsch } & \text { ital. } & \text { italisch } \\ \text { air. } & \text { altirisch } & \text { lat. } & \text { lateinisch } \\ \text { as. } & \text { altsächsisch } & \text { ndän. } & \text { neudänisch } \\ \text { aspan. } & \text { altspanisch } & \text { nengl. } & \text { neuenglisch } \\ \text { avest. } & \text { avestisch } & \text { nhd. } & \text { neuhochdeutsch } \\ \text { awn. } & \text { altwestnordisch } & \text { nisl. } & \text { Neuisländisch } \\ \text { germ. } & \text { germanisch } & \text { nnorw. } & \text { neunorwegisch } \\ \text { got. } & \text { gotisch } & \text { nschwed. } & \text { neuschwedisch } \\ \text { gr. } & \text { altgriechisch } & \text { slov. } & \text { slovenisch } \\ \text { heth. } & \text { hethitisch } & \text { ved. } & \text { vedisch } \\ \text { idg. } & \text { indogermanisch } & \text { westfries. } & \text { neuwestfriesisch }\end{array}$

\section{Literatur}

Baayen, H. (1992): Quantitative aspects of morphological productivity. In: Booij, G., van Marle, J. \& Aronoff, M. (Hg.), Yearbook of Morphology 1991. Dordrecht: Springer. 109-149.

Baayen, H., Dijkstra, T. \& Schreuder, R. (1997): Singulars and Plurals in Dutch: Evidence for a Parallel DualRoute Model. Journal of Memory and Language 37. 94-117.

Bammesberger, A. (1984): Die urgermanischen Aoristpräsentien und ihre indogermanischen Grundlagen. In: Untermann, J. \& Brogyanyi, B. (Hg.), Das Germanische und die Rekonstruktion der Indogermanischen Grundsprache. Akten des Freiburger Kolloquiums der Indogermanischen Gesellschaft, Freiburg, 26.-27. Februar 1981. Amsterdam, Philadelphia: John Benjamins. 1-24.

Bammesberger, A. (1986): Der Aufbau des germanischen Verbalsystems. Heidelberg: Carl Winter. (= Indogermanische Bibliothek. Erste Reihe: Lehr- und Handbücher. Untersuchungen zur vergleichenden Grammatik der germanischen Sprachen, 1).

Bammesberger, A. (1996): The preterite of Germanic strong verbs in classes four and five. North-Western European Language Evolution 27. 33-43.

Barðdal, J. (2008): Productivity. Evidence from case and argument structure in Icelandic. Amsterdam: John Benjamins (= Constructional Approaches to Language, 8).

Bartholomae, Ch. (1885): Die altindischen ē-Formen im schwachen perfekt. Zeitschrift für vergleichende Sprachforschung auf dem Gebiete der indogermanischen Sprachen 27/4. 337-366.

Bauer, L. (1997): A Class of English Irregular Verbs. English Studies 6. 545-555.

Bauer, L. (2001): Morphological productivity. Cambridge, New York: Cambridge University Press (= Cambridge Studies in linguistics, 95).

Boutkan, D. (1995): The Germanic "Auslautgesetze". Amsterdam: Rodopi. (= Leiden Studies in Indo-European, 4).

Braune, W. \& Heidermanns, F. (2004): Gotische Grammatik. Mit Lesestücken und Wörterverzeichnis. Berlin: de Gruyter (= Sammlung kurzer Grammatiken germanischer Dialekte. A: 1). 
Braune, W. \& Reiffenstein, I. (2004): Althochdeutsche Grammatik I. Laut- und Formenlehre. Berlin: de Gruyter (= Sammlung kurzer Grammatiken germanischer Dialekte. A: Hauptreihe, 5/1).

Brugmann, K. (1913): Zu den Ablautverhältnissen der sogenannten starken Verba des Germanischen. Indogermanische Forschungen 32. 179-195.

Bybee, J. (1985): Morphology. A Study of the Relation Between Meaning and Form: John Benjamins Publishing Company (= Typological Studies in Language, 9).

Bybee, J. (1988): Morphology as Lexical Organization. In: Hammond, M. \& Noonan, M. (Hg.), Theoretical Morphology. Approaches in Modern Linguistics. San Diego: Academic Press. 119-141.

Bybee, J. (1995): Regular Morphology and the Lexicon. Language and Cognitive Processes 5/10. 425-455.

Bybee, J. (2010): Language, Usage and Cognition. Cambridge: Cambridge University Press.

Cowgill, W. (1957): The Indo-European long-vowel-preterits. Yale: Yale University.

Dammel, A. (2011): Konjugationsklassenwandel. Prinzipien des Ab-, Um- und Ausbaus verbalflexivischer Allomorphie in germanischen Sprachen. Berlin, New York: de Gruyter (= Studia linguistica Germanica, 103).

Enger, H.-O. (2010): /æprede/ i Gudbrandsdalen - grammatikalisering og homonymiunngåelse? Folkmålsstudier 48. 75-92.

Fertig, D. (2013): Analogy and Morphological Change. Edinburgh: Edinburgh University Press (= Edinburgh historical linguistics).

Gaeta, L. (2006): How to Live Naturally and not be Bothered by Economy. Folia Linguistica 40/1-2. 7-28.

García García, L. (2005): Germanische Kausativbildung. Die deverbalen jan-Verben im Gotischen. Göttingen: Vandenhoeck \& Ruprecht (= Historische Sprachforschung. Ergänzungsheft, 45).

Haspelmath, M. (2006): Against Markedness (And What to Replace it With). Journal of Linguistics 42. 25-70.

Hill, E. (2007): Proportionale Analogie, paradigmatischer Ausgleich und Formerweiterung. Ein Beitrag zur Typologie des morphologischen Wandels. Diachronica 24/1. 81-118.

Hill, E. (2009): Das starke Präteritum der Klasse VII in den nord- und westgermanischen Sprachen. Teil I. International Journal of Diachronic Linguistics and Linguistic Reconstruction 6. 49-123.

Hill, E. (2010): A case study in grammaticalized inflectional morphology. Origin and development of the Germanic weak preterite. Diachronica 27/3. 411-458.

Jasanoff, J. (2012): Long-vowel preterites in Indo-European. In: Melchert, C. (Hg.), The Indo-European Verb. Proceedings of the Conference of the Society for Indo-European Studies, Los Angeles 13-15 September 2010. Wiesbaden: Reichert. 127-135.

Kim, R. (2001): Tocharian B śem $\approx$ Latin vēnit? Szemerényi's Law and $*_{\bar{e}}$ in PIE root aorists. Münchener Studien zur Sprachwissenschaft 61. 119-147.

Kortlandt, F. (1992): The Germanic Fifth Class of Strong Verbs. North-Western European Language Evolution 19. 101-107.

Kortlandt, F. (2004): Accent and ablaut in the Vedic verb. Indo-Iranian Journal 47/1. 7-15.

Kortlandt, F. (2011): Where have all the aorists gone? Amsterdamer Beiträge zur älteren Germanistik 67. 143-148.

Krause, W. (1968): Handbuch des Gotischen. München: C.H. Beck'sche Verlagsbuchhandlung (= Handbücher für das Studium der Germanistik).

Kroonen, G. (2013): Etymological Dictionary of Proto-Germanic. Leiden: Brill (= Leiden Indo-European Etymological Dictionary Series, 11).

Kümmel, M. (2000): Das Perfekt im Indoiranischen. Eine Untersuchung der Form und Funktion einer ererbten Kategorie des Verbums und ihrer Weiterentwicklung in den altindoiranischen Sprachen. Wiesbaden: Reichert.

Kümmel, M. (2004): Zur o-Stufe im indogermanischen Verbalsystem. In: Clackson, J. \& Olsen, B. A. (Hg.), Indo-European Word Formation. Proceedings of the Conference held at the University of Copenhagen October 20th-22th 2000. Kopenhagen: Museum Tuscalanum Press. 139-158.

Langacker, R. (1987): Foundations of Cognitive Grammar. Volume 1. Theoretical Prerequisites. Stanford: Stanford University Press.

Leech, G., Rayson, P. \& Wilson, A. (2001): Word Frequencies in Written and Spoken English. Based on the British National Corpus. Harlow: Longman.

Linell, P. (1977): Knilpor, mejder och snulkar - ett experiment med pluralbildning i svenskan. In: Otterbjörk, R. \& Sjöström, S. (Hg.), Provinsiellt och universellt. En vänskrift till Karl-Hampus Dahlstedt. 19 april 1977. Umeå: Umeå universitet. 91-101.

Ljungstedt, K. (1887): Anmärkningar till det starka preteritum i germanska språk. Uppsala: Akademiska Boktryckeriet.

Lloyd, A., Lühr, R. \& Springer, O. (1998): Etymologisches Wörterbuch des Althochdeutschen. Band 2: bîezzo. Göttingen: Vandenhoeck \& Ruprecht.

Lloyd, P. (1987): From Spanish to Latin. Philadelphia: American Philosophical Society (= Memoirs of the American Philosophical Society held at Philadelphia for Promoting Useful Knowledge, 173). 
Lubotsky, A. (2012): Dissimilatory loss of $i$ in Sanskrit. In: Sukač, R. \& Šefčík, O. (Hg.), The Sound of IndoEuropean 2. Papers on Indo-European Phonetics, Phonemics and Morphophonemics. München: Lincom. 177-181.

Lühr, R. (1984): Reste der athematischen Konjugation in den germanischen Sprachen. In: Untermann, J. \& Brogyanyi, B. (Hg.), Das Germanische und die Rekonstruktion der Indogermanischen Grundsprache. Akten des Freiburger Kolloquiums der Indogermanischen Gesellschaft, Freiburg, 26.-27. Februar 1981. Amsterdam, Philadelphia: John Benjamins. 25-90.

Mailhammer, R. (2007): The Germanic Strong Verbs. Foundations and Development of a New System. Berlin, New York: Mouton de Gruyter (= Trends in Linguistics. Studies and Monographs, 183).

Matzel, K. (1970): Zum System der starken Verben des Germanischen. In: Tilakasiri, J. (Hg.), Añjali: Papers on Indology and Buddhism. A Felicitation Volume Presented to Oliver Hector de Alwis Wijesekera on his Sixtieth Birthday. Peradeniya: The Felicitation Volume Editorial Committee University of Ceylon. 172-181.

Mayrhofer, M. (1978): Sanskrit-Grammatik mit sprachvergleichenden Erläuterungen. Berlin, New York: W. de Gruyter (= Sammlung Göschen, 2207).

Meid, W. (1971): Das germanische Praeteritum. Indogermanische Grundlagen und Ausbreitung im Germanischen. Innsbruck: Institut für Vergleichende Sprachwissenschaft der Universität Innsbruck (= Innsbrucker Beiträge zur Sprachwissenschaft 3).

Meier-Brügger, M. (2010): Indogermanische Sprachwissenschaft. Berlin: Walter de Gruyter (= De Gruyter Studium).

Meiser, G. (2004): Zur Vorgeschichte des lateinischen Perfektsystems. In: Poschenrieder, T. (Hg.), Die Indogermanistik und ihre Anrainer. Dritte Tagung der Vergleichenden Sprachwissenschaftler der Neuen Länder. Stattgehabt an der Ernst-Moritz-Arndt-Universität zu Greifswald in Pommern am 19. und 20. Mai 2000. Innsbruck: Institut für Sprachen und Literaturen der Universität Innsbruck. 191-213.

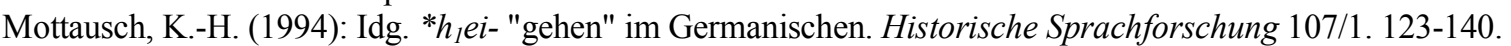

Mottausch, K.-H. (2000): Das Präteritum der 4. und 5. starken Verbklassen im Germanischen. North-Western European Language Evolution 36. 45-58.

Mottausch, K.-H. (2013): Untersuchungen zur Vorgeschichte des germanischen starken Verbs. Die Rolle des Aorists. Hamburg: Kovač (= Schriftenreihe Philologia. Sprachwissenschaftliche Forschungsergebnisse, 173).

Müller, St. (2007): Zum Germanischen aus laryngaltheoretischer Sicht. Mit einer Einführung in die Grundlagen. Berlin, New York: Walter de Gruyter (= Studia linguistica Germanica, 88).

Noreen, A. (1904): Altschwedische Grammatik mit Einschluss des Altgutnischen. Altnordische Grammatik II. Halle: Max Niemeyer (= Sammlung kurzer Grammatiken germanischer Dialekte, A. Hauptreihe VIII).

Nübling, D. (2000): Prinzipien der Irregularisierung. Eine kontrastive Analyse von zehn Verben in zehn germanischen Sprachen. Tübingen: Niemeyer (= Linguistische Arbeiten, 415).

Nübling, D. (2001): The Development of "junk". Irregularization Strategies of HAVE and SAY in the Germanic Languages. In: Booij, G. \& van Marle, J. (Hg.), Yearbook of Morphology 1999. Dordrecht: Kluwer. 53-74.

Nübling, D. (2011): How do Exceptions Arise? On Different Paths to Morphological Irregularity. In: Simon, H. \& Wiese, H. (Hg.), Expecting the Unexpected: Exceptions in Grammar. Berlin, New York: De Gruyter Mouton. 139-162.

Osthoff, H. \& Brugmann, K. (1878): Morphologische Untersuchungen auf dem Gebiete der indogermanischen Sprachen. Erster Theil. Leipzig: Hirzel.

Penke, M. (2006): Flexion im mentalen Lexikon. Tübingen: Niemeyer. (= Linguistische Arbeiten, 503).

Pinker, St. (1999): Words and Rules. The Ingredients of Language. London: Weidenfeld \& Nicolson.

Pinker, St. \& Prince, A. (1992): Regular and Irregular Morphology and the Psychological Status of Rules of Grammar. In: Sutton, L., Johnson, Ch. \& Shields, R. (Hg.), Proceedings of the Seventeenth Annual Meeting of the Berkeley Linguistics Society: General Session and Parasession on the Grammar of Event Structure. Berkeley: Berkeley Linguistics Society. 230-251.

Prokosch, E. (1939): A Comparative Germanic Grammar. Philadelphia: Linguistic Society of America.

Ringe, D. (1998): Probalisitic evidence for Indo-Uralic. In: Salmons, J. \& Joseph, B. (Hg.), Nostratic. Sifting the Evidence. Amsterdam, Philadelphia: John Benjamins. 153-197.

Ringe, D. (2006): From Proto-Indo-European to Proto-Germanic. Oxford: OUP Oxford (= A Linguistic History of English, 1).

Rix, H. \& Kümmel, M. (2001): LIV, Lexikon der indogermanischen Verben. Die Wurzeln und ihre Primärstammbildungen. Wiesbaden: Reichert.

Ruoff, A. (1981): Häufigkeitswörterbuch gesprochener Sprache. Gesondert nach Wortarten, alphabetisch, rückläufig alphabetisch und nach Häufigkeit geordnet. Tübingen: Niemeyer (= Idiomatica, 8).

Schnieders, M. (1938): Die einheimischen nicht komponierten schwachen Verben der jan-Klasse im Altnordischen. Göttingen: Vandenhoeck \& Ruprecht (= Hesperia, 19). 
Schreuder, R. \& Baayen, H. (1995): Modeling morphological processing. In: Feldmann, L. B. (Hg.), Morphological Aspects of Language Processing. Hillsdale: Erlbaum. 131-154.

Schumacher, St. (1998): Eine alte Crux, eine neue Hypothese: gotisch iddja, altenglisch ēode. Die Sprache 40/2. 179-201.

Schumacher, St. (2005): 'Langvokalische Perfekta' in indogermanischen Einzelsprachen und ihr grundsprachlicher Hintergrund. In: Meiser, G. \& Hackstein, O. (Hg.), Sprachkontakt und Sprachwandel. Akten der XI. Fachtagung der Indogermanischen Gesellschaft, 17.-23. September 2000, Halle an der Saale. Wiesbaden: Dr. Ludwig Reichert. 591-626.

Seebold, E. (1970): Vergleichendes und etymologisches Wörterbuch der germanischen starken Verben. Den Haag, Paris: Mouton (= Janua linguarum. Series practica, 85).

Streitberg, W. (1974 [1896]): Urgermanische Grammatik. Einführung in das vergleichende Studium der altgermanischen Dialekte. Heidelberg: Carl Winter Universitätsverlag.

Sverdrup, J. (1927): Der Aorist im germanischen Verbalsystem und die Bildung des starken Präteritums. In: Festskrift til Hjalmar Falk. 30. desember 1927. Fra elever, venner og kolleger. Oslo: Aschehoug \& Co. 296-330.

Tichy, E. (2009): Indogermanistisches Grundwissen für Studierende sprachwissenschaftlicher Disziplinen. Bremen: Hempen.

Voyles, J. (1980): Reduplicating Verbs in North-West Germanic. Lingua 52. 89-123.

Wegera, K.-P. \& Waldenberger, S. (2012): Deutsch diachron. Eine Einführung in den Sprachwandel des Deutschen. Berlin: Erich Schmidt (= Grundlagen der Germanistik, 52).

Wurzel, W. U. (1984): Flexionsmorphologie und Natürlichkeit. Ein Beitrag zur morphologischen Theoriebildung. Berlin: Akademie-Verlag (= Studia grammatica, XXI). 



\title{
Kommunikationsstrategien ungeübter Schreiber - Eine exemplarische Studie anhand eines französischen Bittbriefs
}

\author{
Stephanie Massicot (Erlangen)
}

\begin{abstract}
Der vorliegende Beitrag beschäftigt sich mit der Analyse des Schriftguts sogenannter semicolti, d.h. Personen, die - wenngleich alphabetisiert - nicht täglich mit der Schriftkultur konfrontiert sind und daher auch bei schriftlicher Kommunikation stark in der (konzeptionellen) Mündlichkeit verhaftet bleiben. In der Forschung stand bisher eine - aus der Perspektive einer elaborierten Schriftkultur heraus - eher negative und defizit-orientierte Betrachtungsweise im Vordergrund. Das hier angestrebte Ziel soll es hingegen sein, diejenigen Strategien herauszuarbeiten, derer sich die Autoren im Rahmen ihrer (vergleichsweise eingeschränkten) Versprachlichungsmöglichkeiten bedienen, um ihre jeweiligen kommunikativen Ziele zu erreichen. $\mathrm{Zu}$ diesem Zweck wird beispielhaft eine Analyse eines französischen Bittbriefes auf den verschiedensten sprachlichen ((Ortho-)Graphie, Syntax, Lexik, Vertextungsmuster etc.) und außersprachlichen (äußere Textgestalt, Handschrift etc.) Ebenen durchgeführt. Die Analysen sowie das aus dem 19. Jh. stammende Korpus sind dabei - dies zeigt die folgende Untersuchung - nicht nur von romanistisch-linguistischem Interesse, sondern besitzt - mit dem geschichtswissenschaftlichen Ansatz einer History from below - auch interdisziplinäre Relevanz und bewegt sich damit letztlich an der Schnittstelle zwischen historischer Pragmatik und Textlinguistik, die als theoretischer und methodischer Rahmen fungieren. Zugleich reiht sie sich in die (historische) Substandardforschung ein.
\end{abstract}

\section{Einleitung}

Der Beitrag versteht sich als Versuch, Textzeugnisse ungeübter Schreiber aus einer neuen, positiv ausgerichteten Perspektive zu betrachten. Dabei soll es darum gehen, Kommunikationsstrategien solcher ungeübter Schreiber anhand französischer Texte aus dem 19. Jh. zu betrachten.

Die Fragestellung wird auf die Kommunikationsform und Textsorte 'Brief' zentriert, die medial eindeutig als graphisch zu etikettieren ist, deren konzeptionelle Zuordnung großen Schwankungen unterliegt, was die Untersuchung von Briefen, deren Verfasser in der italianistischen/hispanistischen und auch frankoromanistischen Sprachwissenschaft seit einigen Jahren unter der Bezeichnung semicolti ${ }^{1} /$ semicultos (dt. 'Schreibnovizen', 'Schreibneulin$\mathrm{ge}^{6}$ ) gefasst werden, besonders lohnend macht. Denn es handelt sich dabei um Personen, die zwar alphabetisiert sind, aber nicht die volle Kompetenz im Bereich der Schriftlichkeit erworben haben:

\footnotetext{
1 Nachfolgend soll dieser Terminus auch für die französischsprachigen ungeübten Schreiber benutzt werden, und zwar vor allem deshalb, weil er in der Forschung allgemein etabliert ist und sich nicht auf einzel- bzw. kulturspezifische Phänomene bezieht. Die Frage nach der Adäquatheit des Begriffs sowie eine exhaustive Terminologiegeschichte können nicht Teil dieses Beitrags sein.
} 
[...] les individus mêmes alphabétisés, qui se trouvent en contact direct avec la culture écrite ne sont pas forcément en mesure de profiter pleinement du potentiel de réflexion et de planification offert par une langue élaborée. C'est ce que nous pouvons observer dans les textes produits par ceux qu'on appelle semicolti/semicultos. (Koch \& Oesterreicher 2001: 601; Herv. durch Fettdruck S.M.)

'[...] auch wenn die Individuen, die sich in direktem Kontakt mit der Schriftkultur befinden, alphabetisiert sind, sind sie nicht unbedingt in der Lage, das Reflexions- und Planungspotential einer elaborierten Sprache vollständig zu nutzen. Eine Tatsache, die man in den Texten, die von sogenannten semicolti/semicultos verfasst wurden, feststellen kann. ${ }^{2}$

Ähnliches konstatiert D'Achille, der insbesondere den stark mündlichen Duktus der Texte der semicolti betont:

Esiste, infatti, la categoria particolare dei cosiddetti 'semicolti', i quali, pur essendo alfabetizzati, non hanno acquisito una piena competenza della scrittura e pertanto rimangono sempre legati alla sfera dell'oralità [...]. (D'Achille 1994: 41; Herv. durch Fettdruck S.M.)

'Es gibt nämlich die spezielle Kategorie der sogenannten ,semicolti'; diese haben, wenngleich sie alphabetisiert sind, nicht die volle Schreibkompetenz erworben und bleiben daher immer in der Sphäre der Mündlichkeit verhaftet [...].'

Aus linguistischer Perspektive stellt sich hier die Frage, welche sprachlichen Mittel und welche Kommunikationsstrategien solche ungeübten Schreiber - im Gegensatz zu schreibgewohnten Personen - einsetzen, um ihre Interessen auszudrücken. Und zwar nicht wie bisher auf Basis einer „Defizit“-Orientierung (vgl. z.B. Spitzer 1921; Massariello Merzagora 2008; Fresu 2014), sondern im Hinblick auf die Strategien der Schreiber.

\section{Forschung: Status quo und Desiderata}

Die Sprachwissenschaft beschäftigt sich seit längerem mit dem Schriftgut der semicolti. Betrachtet man den aktuellen Forschungsstand, so steht man mit Blick auf den hispano- und italophonen Raum einer großen Fülle an Bestandsaufnahmen und Beschreibungen des Schriftguts solcher Schreibnovizen gegenüber, während der frankophone Raum bisher deutlich weniger bearbeitet worden ist. Dieser Umstand wird auch in Martyn Lyons' Forschungsüberblick Writing Culture of Ordinary People in Europe, c. 1860-1920 (2013) deutlich, in dem das Französische lediglich im Hinblick auf die Soldatenbriefe des 1. Weltkriegs, der Briefe der poilus, Erwähnung findet.

Die Anfänge der Erforschung des 'Schreibnoviziats'” avant la lettre finden sich, zumindest für das Italienische, bereits in den 20er Jahren des letzten Jahrhunderts mit Leo Spitzer $(1921 / 1976)^{4}$ und seiner Untersuchung zu den Briefen italienischer Kriegsgefangener.

Einen ersten Höhepunkt erlebte die Untersuchung des Schriftguts italienischer Schreibnovizen in den 1980er und 1990er Jahren. In diesem Kontext ist insbesondere Francesco Bruni von zentraler Bedeutung, da er den Terminus semicolti 1978 in die Forschungsdiskussion einführt, und diesen 1984 noch weiter ausarbeitet und zwar im Rahmen des letzten Kapitels

Alle Übersetzungen stammen - soweit nicht anders angegeben - von S.M.

Als begriffliches Gegenstück fungiert hier der - ursprünglich aus der Kognitionspsychologie bzw. Didaktik stammende - Begriff der „Schreibexperten“(vgl. Raible 1995: ix; Stoll 1997: 7)

4 Diese sind auch frei zugänglich online abrufbar unter:

https://archive.org/stream/italienischekrie00spituoft/italienischekrie00spituoft_djvu.txt. 
seiner italienischen Sprachgeschichte, das er verschiedenen Textzeugnissen dieser ungeübten Schreiber, von der Antike bis hin zu den Texten bei Benevolo (1979), widmet. Eine andere Stoßrichtung liegt bei Berruto (1983) vor: Er beschäftigt sich mit sprachlichen Vereinfachungsprozessen bei der Kodierung und Strukturierung, die in den Texten der semicolti anzutreffen sind.

Der aktuelle Standardhandbuchartikel zu dieser Thematik, ebenfalls für den italienischsprachigen Raum, wurde von D'Achille (1994) verfasst, der sich im zweiten Band (Scritto $e$ parlato) der Storia della lingua italiana spezifisch mit den sprachlichen Phänomenen des italiano dei semicolti beschäftigt.

In der Zwischenzeit sind neue Fragestellungen hinzugekommen und so wird mit Enrico Testa (Testa 2014) und seiner Arbeit zum italiano nascosto die Dichotomie zwischen italienischer Kultursprache einerseits und den Dialekten andererseits ${ }^{5}$ in einem Kontinuum zwischen diesen beiden Polen aufgelöst, wobei er den scritture dei semicolti, die er als Ausgangspunkt für den Bereich zwischen den dialektal abgegrenzten Varietäten und der literarischen Normsprache nimmt, einen prominenten Platz einräumt. Die von Testa vorgebrachte Verortung dieses Schriftguts der semicolti im ,Kontinuum‘ spielt auch für die anvisierte Arbeit eine wesentliche Rolle, da sich die Schreiber auch hier Elemente unterschiedlicher Register bedienen.

In der hispanistischen Forschung gehören die Beiträge von Wulf Oesterreicher zur Schreibkompetenz der semicultos in der indianischen Historiographie (Oesterreicher 1994) sowie die Dissertationsschrift von Eva Stoll (Stoll 1997), die sich mit den diskurstraditionellen und textpragmatischen Aspekten von Texten spanischer Konquistadoren als neuer Trägergruppe für Historiographie befasst, zu den Standardwerken. Beide Publikationen antworten dabei auf die Frage nach der Einordnung dieser historiographischen Texte in das von Peter Koch und Wulf Oesterreicher angenommene Kontinuum zwischen kommunikativer Nähe und Distanz (vgl. Koch \& Oesterreicher 2011).

Der frankophone Raum ist hingegen bisher weitgehend unbearbeitet geblieben; abgesehen von wenigen Arbeiten der deutschen Romanistik, in denen man sich vor allen Dingen auf private Korrespondenzen aus der Feder von Soldaten stützt, fehlen systematische Studien und vor allem empirische Untersuchungen anhand von Schreiben der semicolti an offizielle Instanzen.

So erschließen Analysen wie beispielsweise die von Prein (1921) oder Frei (1929) zwar schriftliche Quellen der semicolti, die dann nach und nach in der Linguistik auf Interesse stoßen, doch beschränken sich beide Autoren auf die Textsorte 'Soldatenbrief'. Ebenso verarbeitet Angela Schrott (Schrott 2015) in ihrer Untersuchung eine große Anzahl solcher Unterschichtentexte, wobei auch sie sich auf Briefe aus der Feder von Soldaten und deren Familienangehörigen aus der Zeit um die Wende vom 18. zum 19. Jh. beschränkt und vor allem auf die Mittel zur Herstellung von Nähe eingeht.

Ein aktuelles Forschungsprojekt zu diesem Themengebiet im weitesten Sinne stellt das DFG-Projekt zu einem Corpus Historique du Substandard Français unter der Ägide Harald Thuns dar, doch geht es hier insbesondere um die Erforschung der français régionaux, wobei die Quellenbasis weitgehend bisherigen Traditionen verpflichtet ist und so vor allen Dingen Textsorten wie 'Soldatenbriefe' oder 'Kriegstagebücher' beinhaltet. ${ }^{6}$

Auch Berruto (1983) verweist auf die Rolle der Dialekte und ihren Beitrag zu den dem italiano dei semicolti zugerechneten Phänomenen.

6 Bisher liegen aus diesem Projekt noch kaum Publikationen vor, doch wird aus Thun (2011) klar, dass hier nicht die Strategien der semicolti im Vordergrund stehen, sondern deren Texte als methodische Basis für einen systematischen Entwurfs einer diachronen Untersuchung der français régionaux dienen. 
Weiter liegt, wiederum zum privaten Schriftgut, eine Veröffentlichung Gerhard Ernsts (Ernst 1999) vor, der sich in seiner Analyse zum français populaire écrit der Graphie privater französischer Texte widmet. Er stellt dabei die Frage nach der Sprachform, die aus der Verquickung von medialer Schriftlichkeit, niedriger sozialer Herkunft des Schreibers und der privaten Kommunikationssituation resultiert, geht dabei aber, der gängigen Defizitorientierung gemäß, in erster Linie auf die verschiedensten sprachlichen Verstöße ein.

Für das Französische ist folglich eine eklatante Daten- und Forschungslücke zum Problemkomplex der semicolti zu beklagen, da sich die bisherigen Publikationen meist nur auf private Korrespondenzen und hier vornehmlich auf ,Soldatenbriefe' beziehen. Die angestrebte Untersuchung insbesondere französischer Texte antwortet damit auf ein unzweifelhaftes Desideratum.

Darüber hinaus verdeutlicht die bisherige Orientierung der linguistischen Forschung eine weitere Lücke: Das Gros der genannten Publikationen zielt nämlich vorrangig auf die (scheinbaren) Defizite der Schreiber und deren Verstöße gegen die sprachliche Norm, d.h. es geht um wie auch immer geartete Verstöße gegen die grammatischen und diskurstraditionellen Regeln (,falsche“ oder „fehlende“ Interpunktion, Orthographiefehler, „fehlerhafte“ Syntax, Elemente konzeptioneller Mündlichkeit, Schwierigkeiten bei der Textstrukturierung, nicht-textsortenadäquate Verprachlichungsmuster etc.) bzw. ganz generell um die „Unfähigkeit“ dieser Schreiber, „eine Mitteilung in einen klar formulierten, syntaktisch einwandfreien Satz zu pressen“ (Spitzer 1921/1976: 285). Und bereits der Begriff des semicolto, des „Halbgebildeten“ suggeriert bereits diese „Defizit"-Perspektive, in diese Schreiber zugleich eine gesellschaftliche Marginalisierung erfährt:

Con il termine semicolto si designa lo scrivente che si serve dello strumento linguistico in modo deviante rispetto alla norma corrente, condivisa e accettata, e il cui comportamento linguistico per tale motivo è soggetto a forte stigmatizzazione sociale. (Fresu 2014: 195)

'Mit dem Begriff semicolto wird ein Schreiber bezeichnet, der bei der sprachlichen Mittel von der gängigen, von allen geteilten und akzeptierten Norm abweicht und dessen Sprachverhalten deshalb eine starke gesellschaftliche Stigmatisierung nach sich zieht.'

Dieser Standpunkt rührt vom Erwartungshorizont des Empfängers her, dessen Vorstellungen bezüglich einer ,adäquaten“ und normgerechten Textkonzeption in diesem Schriftgut oftmals nicht eintreffen. Der „semicolte“ Schreiber aber - und dies wurde bisher weitgehend übergangen - macht das Beste aus seiner Situation mit den ihm zur Verfügung stehenden Möglichkeiten der Verschriftlichung.

Insbesondere das Schriftgut italienischer semicolti wurde immer wieder ausführlich behandelt, jedoch unter diesem defizitärem Vorzeichen und meist ohne adäquate Aufarbeitung im Sinne einer Rekontextualisierung, d.h. einer ,ganzheitlichen“ Textbetrachtung (vgl. Raible 1985; Fesenmeier 2005) mit Hilfe von Nachbardisziplinen wie z.B. der Geschichtswissenschaft oder der Soziologie, deren methodische Grundlagen vor allen Dingen der Rekontextualisierung des Schreibprozesses dienen. ${ }^{7}$ Auch diachron-kontrastive Analysen fehlen

Die französischen Dokumente sind dabei auch von interdisziplinärem Forschungsinteresse (z.B. für die Geschichtswissenschaft), denn wenn D'Achille betont, dass es sich bei diesem Schriftgut um ein Phänomen „dal basso“ (1994: 49) handelt und auch Oesterreicher dies in gleicher Weise hervorhebt, so wird evident, dass auch die Bittbriefe in einem größeren, interdisziplinären Rahmen anzusiedeln sind. Als Texte, die eben nicht vorrangig die „große Geschichte“ im Blick haben, sondern vielmehr den Alltag von Strafgefangenen und ihren Angehörigen (bzw. von Migranten) in den Fokus rücken, repräsentieren sie „Geschichte von unten“ (vgl. Oesterreicher 1998: 214) und reihen sich damit ein in eine bereits mit den „Annales-Schulen“ 
weitgehend. Gegenstand und Ziel dieses Beitrags soll daher die Skizze einer ,,positiv“ ausgerichteten Perspektive sein, d.h. eines dezidiert an den Lösungsstrategien der Verfasser für ihre kommunikativen Ziele interessierten Ansatzes, der auch die soziohistorischen Bedingungen mit in den Blick nimmt. Dazu sind, neben einer Rekontextualisierung des Schreibprozesses, diejenigen kommunikativen Strategien im Rahmen der im Korpus systematisch relevanten Sprechhandlung ,Erbitten“ herauszuarbeiten und zu analysieren, die auf der textuell-pragmatischen Makroebene anzutreffen sind; etwaige „Defizite“ auf der Mikroebene können freilich zur Verortung der jeweiligen Sprecher hinsichtlich ihres Alphabetisierungsgrads bzw. ihrer Schreibkompetenz dienen (vgl. unten).

\section{Darstellung der Arbeitshypothese, Ziele und Analyseraster}

Während in der bisherigen Forschung die Defizite des semicolti-Schriftguts (kritisch) analysiert wurden, besteht das hier verfolgte Ziel darin, die Strategien zu identifizieren, mit denen die Verfasser der Briefe versuchen, ihren Sprechakt ${ }^{8}$ der 'Bitte/Aufforderung' erfolgreich zu vollziehen. Denn, so die These, trotz der zweifelsohne begrenzten Kompetenzen im distanzsprachlichen ${ }^{9}$ Bereich, über die diese „,normfernen“ Sprecher verfügen, um ihren Bittbrief in der vom Adressaten möglicherweise erwarteten Form zu verfassen, sind sie dennoch in der Lage, ihr Anliegen zu beschreiben. Obschon vieles auf der Mikroebene (z.B. Art der Verschriftung von Lauten, Orthographie, (Nicht-)Setzung der Diakritika etc.) nicht der sprachlichen Norm entspricht, deren präskriptives Gewicht in Frankreich besonders ausgeprägt ist, kann diese scheinbare Fehlerhaftigkeit, die nicht allein auf Interferenzen aus der konzeptionellen und medialen Mündlichkeit reduziert werden kann, auf der Makroebene (z.B. Ebene der Textstruktur oder Pragmatik) ausgeglichen werden. Die Verschriftungsverfahren der Autoren der hier zugrundeliegenden Texte ${ }^{10}$ - zum Teil angetrieben vom Traum einer besseren Existenz in den fremden und fernen Ländern - zeugen nämlich von zahlreichen Kompensationsstrategien, mit denen sie ihr fehlendes Bewusstsein für Verwendungsmöglichkeiten der sprachlichen und - spezifischer - diskurstraditionellen ${ }^{11}$ Ausdruckmittel „Bittbrief“ ausglei-

der 1960er Jahre beginnende, historiographische Diskussion einer „Geschichte des Alltags“, einer History from Below (vgl. Thompson 1996).

Auf die Historizität von Sprechakten verweist u.a. Gudrun Held: „Es dürfte wohl keine Frage sein, dass Sprechakte und v.a. die Art der Realisierung kulturspezifisch und damit historisch sind: So gibt es heute bestimmte Sprechakte wie bannen, ächten, verfluchen nicht mehr oder in anderer Form als früher; grüßen, danken oder eine Liebeserklärung machen werden anders vollzogen als in vergangenen Jahrhunderten" (Held 2006: 2312).

9 Diese Bezeichnung wird hier verstanden im Sinne von Koch \& Oesterreicher; sie sprechen in Anlehnung an Sölls Unterscheidung in Bezug auf die konzeptionelle Mündlichkeit auch von „Nähe“ und in Bezug auf die konzeptionelle Schriftlichkeit von „Distanz“: „Es lässt sich also sagen: Die beiden oben beschriebenen Extrempole des, gesprochen/geschrieben'-Kontinuums entsprechen Kommunikationsformen, die in allen Parametern einerseits maximale kommunikative Nähe (,gesprochen'), andererseits maximale kommunikative Distanz (,geschrieben') verkörpern. In dem vieldimensionalen Raum, der von diesen beiden extremen Formen sprachlicher Kommunikation begrenzt wird, sind alle konzeptionellen Möglichkeiten zwischen ,Mündlichkeit" und ,Schriftlichkeit" lokalisierbar" (Koch \& Oesterreicher 2011: 10). Nach Meinung der beiden Autoren umfasst das von beiden Polen abgeschlossene konzeptionelle ,Nähe/Distanz-Kontinuum“ eine Vielzahl von unterschiedlichen Kommunikationskonstellationen bzw. Bedingungen, wie beispielsweise den Grad an Öffentlichkeit der Kommunikation, den Grad an Spontaneität bzw. Reflektiertheit, den Grad der emotionalen Beteiligung etc. (Koch \& Oesterreicher 2011: 13).

10 Vgl. hierzu Kapitel 4 vorliegender Publikation.

11 „Eine Text- oder Diskurstradition entspricht in diesem Sinne der Gesamtheit der Äußerungen einer bestimmten Textsorte (oder eines Diskurstyps) über die Zeiten hinweg. Zu einem gegebenen Zeitpunkt entsprechen der Diskurstradition definierbare Diskursnormen und -regeln, die jeweils die Grundmuster der 
chen können, sodass der direktive Sprechakt ,Erbitten', der den Adressaten des Briefs zu einer bestimmten Handlung auffordert, letztendlich glückt (vgl. unten).

Um nun die Kommunikationsstrategien der Schreiber nicht, wie bisher üblich, allein als Abweichungen von der Norm zu untersuchen, sondern vor allem unter dem ,positiven“ Vorzeichen einer anderen Art und Weise der Textorganisation, sollen unterschiedliche Ebenen der Textanalyse betrachtet werden, die sich wie folgt aufgliedern lassen:

1. Paratextuelle Ebene

1.1. Materielle Textgestalt

- Papier (z.B. Vordruck, Stempel)

- Aufbewahrung (Zustand)

- Schreibwerkzeug

1.2. Äußere Textgestalt

- Äußere Textgliederung (Absätze, Unterschrift, Paginierung)

- Nebentext (z.B. Randnotizen)

- Einhaltung der Zeilenlinien und Zeilenabstände

- Art der Handschrift (z.B. sicher vs. unsicher)

- Revisionen (z.B. Streichungen, Verbesserungen)

- Lesbarkeit

2. Sprachliche Ebene

2.1.(Ortho-)Graphische Auffälligkeiten

- Groß-Kleinschreibung

- Graphie-Phonie-Relation

- Interpunktion

2.2. Morphosyntaktische Besonderheiten

2.3. Lexikalische Charakteristika

2.4. Textstrukturelle und pragmatische Ebene

2.5. Diskurstraditionelle Ebene

- Textaufbau: Makrostruktur

- Verhältnis: Formelhaftigkeit vs. „freier“ Teil

Sowohl die Untersuchung der paratextuellen als auch die der sprachlichen Ebene sind hier von zentraler Bedeutung, da der Ausgangspunkt die - auf den ersten Blick banal wirkende Beobachtung ist, dass nicht nur sprachliche, sondern auch paratextuelle Phänomene wertvolle Hinweise für die Einordnung des Schriftguts in den soziohistorischen Kontext, die Situierung der Sprechsituation sowie auch die Frage nach dem Bildungsgrad des Sprechers (Stichwort 'semicolti') geben können.

\section{Korpusvorstellung Les lettres des bagnards et leurs proches}

\subsection{Vorbemerkungen}

Die Herausarbeitung der Strategien der französischen semicolti wird sich, wie erwähnt, auf französische Bittbriefe stützen, ${ }^{12}$ von denen einer für den vorliegenden Beitrag zur Illustration

zugehörigen Textsorten prägen; die diachrone Veränderung dieser Normen wird dann zur Tradition“ (Gleßgen 2005: 209).

12 Das Korpus umfasst ca. 300 Briefe, die bereits in vorläufiger Form transkribiert sind und nun editionsphilologisch aufbereitet werden. Als „historische Überreste“ (vgl. Droysen 1977/1882: 26) sind sie geprägt 
der Phänomene und des Analyseverfahrens ausgewählt wurde (vgl. Anhang). Die Untersuchung dieser handschriftlich verfassten Texte liefert zunächst paläographische Daten, wobei die daraus resultierenden Ergebnisse, wie z.B. das Schriftbild, die Einhaltung der Zeilen etc. für die soziale Lokalisierung der Handschrift sowie für die Aufbereitung des Korpus äußerst wertvoll sind. Aus diesem Grund ist hier stets eine ganzheitliche Betrachtung des Texts anzustreben ist - und zwar auch im Sinne einer Rekontextualisierung des Schreibprozesses (vgl. „recontextualisation“ bei Fleischman 1990: 37; Oesterreicher 2005).

\subsection{Die dossiers der Archives nationales d'outre-mer}

Das Korpus stellt nun aus mehreren Gründen einen „Glücksfall“ dar: Zum einen deshalb, weil in Frankreich nur das, was die „große“ Geschichte tangierte, als aufbewahrungswürdig erschien, so dass hier - im Gegensatz zu Spanien oder Italien - keine spezialisierten Archive für Alltagsgeschichte existieren. Dies macht eine systematische Suche nach Dokumenten solcher „Schreibnovizen“ praktisch unmöglich und deren Erhalt besonders erstaunlich. Auch weil diese Schreiber sich - als verurteilte Gefängnisinsassen - stets in einer gesellschaftlichen Marginalposition befanden.

Zum anderen handelt es sich auch deswegen um einen Glücksfall, weil diese originalen Texte auch wertvolle paläographische Daten enthalten, die z.B. für die soziale Verortung der Handschrift sehr wertvoll sind.

All diese Briefe der bagnards ${ }^{13}$ bzw. ihrer Angehörigen sind gegen Mitte des 19. Jh. und des beginnenden 20 . Jh. entstanden. Sie lagerten - bisher weitgehend unbekannt - im Nationalarchiv für überseeische Gebiete (Archives nationales d'outre-mer) in Aix-en-Provence. Bei den dortigen Recherchen für vorliegende Untersuchung wurden unterschiedliche Repertorien, d.h. Findbücher, konsultiert, die anhand bestimmter Selektionskriterien ausgewählt wurden.

Zum einen wurde eine zeitliche Einschränkung vorgenommen. Aus historischen Gründen standen und stehen diejenigen Dokumente im Zentrum, die zwischen 1852 und 1896 entstanden sind. Und zwar aus dem Grund, weil die flächendeckende Schulpflicht in Frankreich mit den sogenannten lois Ferry zu Beginn der 1880er Jahre eingeführt wurde (école publique, laique et obligatoire). Die Wahrscheinlichkeit, in dieser Zeit Schriftgut von semicolti zu finden, stieg damit natürlich.

Zudem war für die Auswahl auch die hierarchisch klar gegliederte Beziehung zwischen Schreiber und Empfänger von größter Bedeutung. Ein solch asymmetrisches Beziehungsgeflecht veranlasst die semicolti dazu, sich eines sprachlichen Registers zu bedienen, mit dem sie nur wenig vertraut sind. Im Zusammenhang mit der Bedeutung der Beziehung zwischen Rezipienten und Emittenten während der sprachlichen Handlung rückt ein weiteres, hier zentrales Element in den Fokus der Betrachtung: das Konzept der (pragmatischen) Höflichkeit. ${ }^{14}$ Daher wurde hier die Sammlung H der „fonds ministériels“ gewählt, in der sich insbesondere die Korrespondenzen zwischen staatlich-ministerialen Amtsträgern (Minister, Präfekt, Direktor) und den meist straffälligen Privatpersonen bzw. deren Angehörigen befinden.

von den Unabwägbarkeiten der Überlieferung und weisen oft schwer rekonstruierbare Lücken auf, beispielsweise in Form von Löchern im Papier oder Wasserflecken, die die Analyse erschweren.

13 Dieser - aus dem Italienischen entlehnte Begriff (vgl. ital. il bagno 'das Bad') - bezeichnete ursprünglich die engen Käfige, in der die Verurteilten auf der Überfahrt gepfercht wurden (vgl. Dion \& Taillemite 2007: 10).

14 Höflichkeit stellt - und dies wird u.a. durch die Begriffsgeschichte augenfällig - eine historische Größe dar und steht damit in unmittelbarem Zusammenhang mit der historischen Pragmatik (vgl. Held 2006: 2305). 
An dieser Stelle soll das Wort einem der Schreiber selbst erteilt werden, der sich in einem Metakommentar zur Schreibsituation äußert:

pardonner moi Monsieur le ministre//si si ne tourne pas par lettre mieux//je ne sais pas comme on doit vous écrire (Lettre Bernard ANOM FM H71)

'Herr Minister, entschuldigen Sie, wenn ich mich nicht mit einem besseren Brief an sie wende, ich weiß nicht, wie man Ihnen schreiben muss'

Diese Einschätzung der Situation ist insofern sehr interessant, als sich der Schreiber bzw. in diesem Fall die Schreiberin bewusst ist, dass sie eben nicht über die der Kommunikationssituation mit dem Minister angemessene Schreibkompetenz im distanzsprachlichem Bereich verfügt.

\subsection{Historische Einordnung}

Es ist nun im Sinne der Rekontextualisierung ${ }^{15}$ des Schreibprozesses wichtig, auch ein Bild des soziohistorischen Kontexts zu erstellen, in dem diese Briefe entstanden sind. Ganz allgemein lassen sich die Bittbriefe eingliedern in die Kolonial- und Strafverfolgungsgeschichte Frankreichs.

Die bagnes coloniaux, also die Arbeitsstrafanstalten in den französischen Kolonien wurden unter dem letzten französischen Kaiser, Louis Napoleon III. Bonaparte, geschaffen. Diese Art der kolonialen Strafverwahrung wird durch ein Gesetz im Jahr 1848 eingeleitet und dann während des Second Empire sowie in der III ème République weiter ausgebaut.

Als die Verfassung von 1848 die Todesstrafe für die politischen Gefangenen abschafft, benötigt man eine Ersatzstrafe. Diese wird in einem Gesetz von 1848 beschrieben als „la déportation dans une enceinte fortifiée, hors du territoire continental de la République“" (zit. nach Dion \& Taillemite 2007: 7)

Deshalb werden die Aufständischen der bürgerlich-demokratischen Februarrevolution von 1848 zunächst nach Algerien und ab 1852 nach Guyana als überseeische Strafkolonie transportiert. Neben dieser Strafkolonie in Guyana gab es ab 1863 auch ein bagne in Neu-Kaledonien. Dorthin wurden, nach der Niederschlagung der revolutionären Aufstände der Commune de Paris 1871, die politisch Verurteilten gebracht - wobei ein großer Teil der Briefe aus dieser Zeit stammt. ${ }^{16}$

\subsection{Diskurstradition 'Bittbrief' und Sprechakt 'Bitte'}

Die Diskurstradition 'Bittbrief' und der Sprechakt 'Bitte' sind aus vielerlei Hinsicht interessant. Die Bitte gilt als Abbild der Sprechhandlung 'Aufforderung' par excellence und ist damit eo ipso ein face-threatening-act. Die Grundlage hierfür bildet die bekannte Theorie der

15 Held bezeichnet die „Rekonstruktion von Kommunikationsformen als Hauptziel“ (Held 2006: 2304) der Historischen Pragmatik.

16 Insgesamt wurden in der Zeit zwischen 1852-1938, d.h. bis zur Abschaffung der bagnes, ungefähr 100.000 Männer und 2000 Frauen in die Strafkolonien versetzt. Viele von ihnen mussten bereits auf der Überfahrt, die eineinhalb Monate dauerte, ihr Leben lassen. Das Leben in den Kolonien war nicht besser, ein großer Teil der Sträflinge wurde krank und verstarb nach nur weniger Zeit. Diejenigen, die es schafften, die Hälfte ihrer Strafe bei guter Führung abzusitzen, konnten jedoch auch ihre Familien zu sich holen, was auch in den Bittbriefen als Anliegen immer wieder zum Ausdruck kommt (vgl. Dion \& Taillemite 2007: 7-19). 
Höflichkeit auf der Grundlage von Brown \& Levinson (1978), Leech (1983) und Goffmann (1974/1971), wonach sich die Kommunikationspartner an den sogenannten face-Bedürfnissen orientieren. Zentral ist hierbei nicht nur die Tatsache, dass das face als öffentliches Selbstbild erst durch Interaktion generiert wird, sondern auch die Erweiterung dieses Konzepts im Sinn einer Verbindung aus einem positiven und einem negativen Gesicht, das die Individuen bei jeglicher Art von Interaktion gelten machen wollen. Das positive face meint dabei ein Bedürfnis nach individueller Wertschätzung, während sich das negative face auf die Wahrung des persönlichen Handlungsfreiraums bezieht (vgl. Brown \& Levinson 1987: 67). Die Autoren der Bittbriefe stehen - angesichts ihres Gesuchs und dem damit verbundenen willentlichen Eindringen in die Handlungsfreiheit des Adressaten als einer Bedrohung des negativen Gesichts - unter einer Art Submissionszwang, der sich in ritualisierten politeness-strategies manifestiert. Es ist also hier sogenanntes face-work $^{17}$ notwendig und damit stehen sich Rhetorik und Pragmatik (direktiver Sprechakt der 'Bitte') gewissermaßen diametral gegenüber, was eine linguistische Analyse besonders lohnend macht, da der hier zugrundeliegende direktive Sprechakt einen Übergriff auf das Territorium des anderen beinhaltet, der sprachlich aufbereitet werden muss. Relevant ist in diesem Zusammenhang, dass - wie erwähnt - ein stark hierarchisiertes Beziehungsgeflecht vorliegt, bei dem der Adressat über dem Sender steht. Hieraus resultieren Regelungen der ALTER-Erhöhung und EGO-Erniedrigung, die durch bestimmte Ehrerbietungs- und Unterwerfungsgesten zum Ausdruck kommen (vgl. Held 2005: 52); so heißt es beispielsweise in den Briefen je me jette à vos pieds 'ich werfe mich zu Ihren Füßen' (Lettre: Le Folle ANOM FM H265) oder auch je suis votre très humble et très devoué serviteur 'ich bin ihr untergebenster und demütigster Diener' (Lettre: Massi ANOM FM H258).

Um zu verstehen, ob die hier untersuchten Briefe der Textsorte des 'traditionellen' Bittbriefs entsprechen, bedarf es erst einer kurzen Klärung, was man grosso modo darunter versteht; und dies insbesondere im Hinblick auf die textstrukturelle und diskurstraditionelle Analyse. Die Textgattung 'Brief' bezieht sich seit dem Mittelalter auf zahlreiche Elemente der sogenannten ars dictaminis, als Lehre vom stilgerechten Abfassen von Briefen, die sich stark an die antike Rhetorik anlehnt (vgl. Held 2005: 46). Diese ars besitzt die idealtypische Makrostruktur: salutatio - exordium - narratio - petitio - conclusio, ${ }^{18}$ wobei auch die Briefsteller ${ }^{19}$ ab dem 16. Jh. Anleihen an diesem Schema nehmen - ohne dies immer explizit zu kennzeichnen (vgl. Große 2003: 136, 147).

Auch in den untersuchten Briefen scheinen diese Elemente der ars dictaminis vermittelt durch verschiedene Briefsteller des 18. und 19. Jh. ${ }^{20}$ immer wieder durch. Als einige zentrale Modelle zum Abfassen offizieller Schreiben wären für die hier untersuchte Zeit beispiels-

17 Unter dem Begriff versteht Goffmann „the positive value a person effectively claims for himself by the line others assume he has taken during a particular contact. Face is an image of self-delineated in terms of approved social attributes-albeit an image that others may share, as when a person makes a good showing for his profession or religion by making a good showing for himself" (Goffmann 2008/1967: 5).

18 In Anlehnung daran gliedert Held (2008: 360f) den 'Bittbrief' in eine

1) Vorbereitungsphase, in der der Schreiber den Kontakt zum Adressaten herstellt und die Sprechhandlung, in diesem Fall die Bitte antizipiert,

2) in die eigentliche Sprechhandlung als zentrale Phase, da hier die Illokution 'Bitte' stattfindet

3) sowie in illokutionsstützende Phasen, sogenannte supportives, die der Rechtfertigung dienen und in denen bestimmte höfliche Ausgleichverfahren angewandt werden.

19 Es handelt sich hier um Anleitungen und Modelle zum Abfassen unterschiedlicher Brieftypen, die stilistische, sprachliche sowie textpragmatische Regeln beinhalten. Im Französischen werden diese mit Begriffen wie secrétaire, manuel épistolaire, manuel de correspondence oder modèles de lettre wiedergegeben (vgl. Große 2003: 135, 138f.). 
weise Le Secrétaire de la cour impériale de France von 1813 oder der Code épistolaire, contenant les règles, les principes et le cérémonial du style épistolaire, avec de modèles de lettres sur toute espèce de sujets von 1829 zu nennen. Problematisch ist in jedem Fall die Frage nach der genauen Beeinflussung auf die untersuchten Briefe und Briefsteller, zumal die Verfasser der Briefe auch nicht mehr befragt werden können; treffend formuliert dies Bax (1991: 200): „die Kommunikationsgemeinschaften, die untersucht werden sollen, sind eben tot und haben ihre Sprache mit ins Grab genommen“".

Dennoch erscheinen einzelne Elemente der Briefsteller immer wieder auf; wenngleich in abgewandelter Form, sodass der Aufbau dieser Briefe in vielen Fällen, als „kreisförmig“ zu etikettieren ist (vgl. unten).

\section{Exemplarische Analyse}

\subsection{Vorbemerkungen}

Wenngleich das Korpus sprechakttheoretisch als homogen ('Bitte') bezeichnet werden kann, lassen sich die untersuchten Bittbriefe inhaltlich in fünf große Bereiche einteilen:

1) Die Bitte der verurteilten Gefängnisinsassen um eine Versetzung in eine der Kolonien

2) Die Bitte der Frauen, ihren Männern ins koloniale Exil folgen zu dürfen

3) Die Bitte von Familienmitgliedern um einen Auszug aus der Sterbeurkunde

4) Bitten von Familienmitgliedern und Bekannten um Neuigkeiten zu ihren Angehörigen

5) Sonstige Themen: Bitten um Geld, um erneute Überprüfung der Tatbestände, Hilfe bei der Zustellung von Briefen an Angehörige, etc.

Die Analyse des folgenden Briefs, in der die theoretischen Anmerkungen bzgl. der in Kapitel 4.4. vorgestellten Analyseebenen veranschaulichen werden sollen, bezieht sich auf den Inhaltspunkt 4), also „Neuigkeiten“. Im Folgenden soll jedoch keine exhaustive Analyse durchgeführt werden. Vielmehr geht es darum, einige zentrale Punkte herauszugreifen, damit ein erster Eindruck entstehen kann.

Wie die wörtliche Abschrift des Briefs (Original vgl. Anhang) illustriert, ist der Brief, was die rein materielle Textgestalt (paratextuelle Ebene) anbelangt, relativ unversehrt. Eine erste, rein äußerliche Textgliederung ist insofern gegeben, als Datum (Le 18 juillet 1873 'Den 18. Juli 1873, Z. 1) und Unterschrift (femme bloquel 'Frau Bloquel', Z. 20) zum einen vorhanden sind und - zumindest was die Briefdatierung angeht - zum anderen auch ein semantisch begründeter Absatz existiert, wie dies auch nach der „Anrede“ des Adressaten (Ministère de la Marine 'Marineministerium', Z. 3) der Fall ist. Der Haupttext wird allerdings en bloc verfasst. Zudem liegen Randnotizen vor (Bloquel, 7639; A été invitée a sePresenter au bureau 23 Juillet 1873 'Bloquel, 7639; Wurde am 23 Juli 1873 eingeladen, im (Ministerial-)Büro vorstellig zu werden'), die aus perlokutive Relevanz besitzen, da sie Aussagen über das Gelingen des Sprechakts zulassen; so erfahren wir, dass die Bittstellerin ins Ministerium geladen wurde - ihrem Gesuch also - zumindest partiell - stattgegeben wurde. Bezüglich der Zeileneinhaltung bzw. des Zeilenabstands zeigt die Autorin zwar keine vollständige Sicherheit, da sich der Zeilenabstand sowie auch die Schriftgröße gegen Ende einer Seite verkleinern, was darauf hindeutet, dass die Verfasserin - im Gegensatz zu den Schreibexperten - nicht von Anfang einschätzen kann, wie viel Platz sie zum Schreiben bei der gewählten Schriftgröße benötigt. Dennoch ist das Schriftbild gut lesbar und klar; und auch die Handschrift als solche erscheint relativ sicher, d.h. die Autorin schreibt ohne saliente individuelle Fluktuationen. $\mathrm{Zu}$ - 
dem sind weder Streichungen noch Einfügungen oder sonstige Revisionen vorhanden, was ebenfalls auf eine nicht völlig ungeübte Hand schließen lässt.

Diese kurze Analyse der paratextuellen Ebene hat gezeigt, dass es sich bei der Autorin um eine Schreiberin handeln dürfte, die der Definition des semicolto entspricht: Sie ist zwar alphabetisiert und besitzt zudem eine relativ sichere Handschrift, dennoch hält sie die Zeilen(abstände) nicht immer ein und benutzt kaum strukturierende Absätze. Es handelt sich also hier um ein Faktum, das zwar zunächst das Lesen etwas erschwert, sich aber letztlich nicht auf das Verständnis auswirkt.

\subsection{Sprachliche Ebene: (Ortho-)Graphische Ebene}

Wenn hier zunächst die sprachliche Ebene betrachtet wird, dann erfolgt dies, wie erwähnt, um diejenigen Merkmale herauszuarbeiten, die für die „Halbgebildetheit“ der Verfasserin sprechen, aber das Verstehen nicht gefährden, was ja als Prämisse für einen erfolgreichen Sprechakt gilt. Die nachfolgenden (Einzel-)Resultate müssen dabei nicht nur isoliert, sondern nach der Gesamtuntersuchung auch summativ betrachtet werden.

\subsubsection{Resyllabierung}

Nach der paratextuellen Ebene soll nun auch die sprachliche Ebene betrachtet werden, wobei sich die Untersuchung auf einige ausgewählte, besonders augenfällige Phänomene beschränkt. Betrachtet man die Graphie-Phonie-Relation, so ist im genannten Textbeispiel das massierte Auftreten an Resyllabierungen besonders markant. Die Wortgrenzen sind instabil und es kommt zu zahlreichen Agglutinationen, meist bedingt durch das Fehlen des Apostrophs. Diese treten insbesondere in der Verbindung „Artikel + Nomen“ oder „Pronomen + Verb“ auf, ${ }^{21}$ wie folgende Beispiele deutlich zeigen: laupitale (Z.7; 'l'hôpital', 'das Krankenhaus'), jai (Z.6; 'j'ai', 'ich habe'), quil mécrié quil (Z.7; 'qu'il m’écrie qu'il', 'dass er mir schrieb, dass'), je serait (Z.8f.; 'je serais', 'ich wäre'), sappelle (Z.13; 's'appelle', 'er heißt'), engrase (Z.15; 'en grâce', 'die Gunst/Gnade'). Fernerhin treten auch Deglutinationen auf, die nur zum Teil etymologisch bedingt sind: et tez (Z.4; 'etiez', 'Sie wären') de puis (Z.7f.; 'depuis', 'seit'), à voir (Z.9; 'avoir', 'haben'), est mable (Z.10; 'aimbale', 'liebenswert'), re mercirait (Z.11; 'remercierais', 'ich würde danken'), vou loire (Z.16; 'vouloir', 'wollen') oder auch par ti (Z.14; 'parti', 'weggegangen'). ${ }^{22}$

\subsubsection{Phonographematisches Prinzip}

Im Französischen gibt es eine ganze Reihe von Graphemkombinationen, die ein eigenständiges Phonem darstellen; ein- und dasselbe Phonem kann also durch unterschiedliche, phonetisch aber gleichgestellte, Graphemgruppen dargestellt werden, wobei sich im Laufe der Sprachgeschichte jeweils eine Schreibweise als normierte und damit schließlich korrekte durchsetzt. Eine solch normierte Schreibung habe sich - so Catach (1995: 1101-1111) -

21 Dazu tauchen im Gesamtkorpus auch immer wieder Agglutinationen auf, die aus der Schrift bzw. aus der Art des Schreibens resultieren, da aus der gleichen Federfüllung mehrere Wörter geschrieben werden; ein Absetzen der Schreibfeder scheint vielfach unökonomisch zu sein.

22 Diese Tendenz verweist dabei auf ein sehr ausgeprägtes Silbenverständnis der Autorin des Briefs. 
bereits im beginnenden 17. Jh. durchgesetzt, was insbesondere mit dem Buchdruck und der damit verbundenen Verbreitung von Wörterbüchern zusammenhänge. Allerdings weist der vorliegende Brief vielfach Verstöße gegen eine solche allgemein verbindliche Norm auf, sodass in jedem Fall davon auszugehen ist, dass selbst am Ende des 19. Jh. mehrere (Graphem-)Varianten für ein- und denselben Laut existieren. Wenngleich eine vollständige Systematik in diesem Rahmen nicht möglich ist, seien dennoch einige Fälle einer solchen phonographematischen Realisierung herausgegriffen, die teilweise auch Auswirkungen auf morphologischer Ebene haben und zum Teil durch das Verstummen des auslautenden Konsonanten im Französischen bedingt sind: donnez (Z.4; 2. Ps.Pl. 'ihr gebt') statt donner (Infinitiv 'zu geben'), nouvelle ${ }^{23}$ (Z.5; Sg. 'Neuigkeit') statt nouvelles (Pl. 'Neuigkeiten'), maris (Z.12) statt mari ('Ehemann'), lètre (Z.6) statt lettre ('Brief'), laupitale (Z.7) statt l'hôpitale ('Krankenhaus'), (je) serait (Z.8; 1. Ps. Sg. '(ich) wäre') statt (je) serais (1. Ps. Sg. 'ich wäre'), mois (Z.12; 'Monat') statt moi ('ich'). Letztlich sind aber diese Normabweichungen lediglich graphischer Natur und für das Verständnis irrelevant, da die Bedeutung durch den Ko(n)text erschlossen werden kann. Dennoch nehmen sie, wie bereits mehrfach erwähnt, für die vorliegende Untersuchung eine Schlüsselrolle ein, da eine solch normferne Schreibung Aufschluss über die Vertrautheit der Schreiber/innen mit Schrift- und Schriftkultur gibt und somit die Grundlage für die Prämisse des Beitrags liefert.

\subsubsection{Groß- und Kleinschreibung}

Der Gebrauch von Majuskeln bzw. Minuskeln hat bekanntermaßen unterschiedliche Funktionen, die cum grano salis wie folgt eingeteilt werden können: a) Aufzeigen eines neuen Satzes oder Abschnitts, einer neuen Wortart (rein funktionell), b) Markierung einer höflichen Distanz, c) Betonung und Hervorhebung von als subjektiv wichtig Eingestuftem oder d) ästhetische-dekorative Funktion ${ }^{24}$ (vgl. u.a. Ernst 1999: 97).

Solche Fälle von Großschreibung sind bei Bittschriften besonders frequent, wie die nachfolgenden Beispiele aus dem analysierten Bittbrief belegen, wobei man es hier mit einer relativ unsystematischen Setzung von Großbuchstaben zu tun hat. Die Anrede Maissieur 'Herr/Herren', ${ }^{25}$ wird immer großgeschrieben, wobei offen bleiben muss, ob dies aufgrund eines bestimmten Normbewusstseins geschieht (a), oder, weil die Autorin dadurch die Wichtigkeit des Empfängers ausdrücken will (c) und zugleich eine höfliche Distanz schafft (b) - oder ob es sich um eine Kombination dieser Funktionen handelt. In jedem Fall bleibt die Autorin aber damit der Diskurstradition 'Bittbrief' treu und verleiht ihrem Sprechakt ('Erbitten') durch diese Schreibung als eine Art „Ehrerbietungsgeste“ - ganz im Sinne der Höflichkeitsmaxime - Nachdruck. Auch der Eigenname des Schiffs (Calvados, Z.13) sowie die Toponyme Calidonie 'Kaledonien' (Z.13) oder Maronne 'Maronne' (Z.17) werden

23 Diese fehlende Pluralmarkierung ist der orthographegrammaticale geschuldet, das heißt, dem ,im Französischen besonders stark ausgeprägten Phänomen, daß - als Folge des Schwunds von Endkonsonanten in der Aussprache - grammatische Markierungen am Wortende nicht phonisch, sondern nur graphisch markiert werden“ (Ernst 1999: 108).

24 Wichtig ist in diesem Zusammenhang, dass sich diese Funktionen nicht immer gegenseitig ausschließen und daher nicht isoliert voneinander zu betrachten sind; so kann beispielsweise in manchen Fällen die dekorative Funktion eng mit dem Unterstreichen von subjektiv als bedeutend wahrgenommenen Sachverhalten korrelieren.

25 Diese Graphie verweist darauf, dass die Autorin wohl mehrere Personen anspricht, da das Maissieur (Z. 4, 8) als nicht-normkonforme und diatopisch oder zumindest diaphasisch/diastratisch markierte Realisierung von Messieurs angesehen werden kann. 
normgerecht groß geschrieben. Gleichzeitig, und dies verweist auf die fehlende Normvorstellung und die subjektive und recht willkürliche Verwendung von Majuskeln/Minuskeln, werden die Eigennamen (bloquel 'Bloquel', Z. 20; benoit 'Benoît', Z. 13 sowie auch nouvelle 'neu', Z. 5 als Bestandteil des Toponyms Nouvelle-Calédonie 'Neu-Kaledonien') klein geschrieben. Das Possessivum in Mon maris 'Mein Ehemann' (Z.12) hingegen wird - entgegen der bestehenden Norm - groß geschrieben, wobei hier als Grund die subjektive Wichtigkeit (c) im Sinne einer Art kommunikativer Salienz angenommen werden kann. Prima vista scheint die Groß-/Kleinschreibung aleatorisch. Trotzdem dürfen zwei Dinge nicht vergessen werden: Das Gesagte ist dennoch verständlich und die veränderte Schreibung ist zum Teil sogar dem Sprechakt dienlich und somit funktionalisiert - so beispielsweise im letzten Fall, bei dem die Majuskel von Mon 'Mein', dem Adressaten die Wichtigkeit ihres Ehemanns betont und damit ihrer Bitte eine zusätzliche Rechtfertigung verleihen kann.

\subsection{Syntaktische Besonderheiten: Dislokation}

Die Analyse der syntaktischen Besonderheiten des Texts rückt einen interessanten Fall auf informationsstruktureller Ebene in den Fokus, wenn die Autorin schreibt: Maissieur je serait si heureuse dan navoir de ses nouvelle 'Herr, ich wäre so froh welche zu haben, von seinen Neuigkeiten' (Z. 8f). Diese Rechtsdislokation, bei der die Objekt- Nominalgruppe (de ses nouvelle) zunächst nur pronominal (dan) repräsentiert ist, hebt sich durch die Rhema-ThemaAnordnung von der unmarkierten Grundserialisierung des französischen Aussagesatzes (Adverbiale Ergänzung - Subjekt - Prädikat - Objekt) ab, sodass das Element ses nouvelles hervorgehoben wird (vgl. hierzu Stark 2008: 312 f.). Diese Konstruktion kann als prototypisches Beispiel für die syntagmatische Anordnung der Nähesprache gesehen werden, in der solche Rhema-Thema-Muster typisch sind (vgl. Koch \& Oesterreicher 2011: 96). Wenngleich es sich hierbei um eine konzeptionell mündliche Syntax handelt, so ist das Gesagte freilich nicht unverständlich, da - im Gegenteil - die Bitte durch die wiederholte Aufnahme des Gesuchs, noch mehr Nachdruck erhält, obschon eine solche Anordnung freilich nicht dem Aptum des offiziellen Bittbriefs entspricht.

\subsection{Textstrukturelle und pragmatische Ebene: Kohäsion und Kohärenz}

Blickt man auf die Ebene der Textkohäsion bzw. -kohärenz, so scheint eine Stelle besonders relevant zu sein; und es liegt prima vista ein Bruch in der Textstruktur vor, da der topicWechsel nicht markiert ist, wenn die Autorin argumentiert:

je vous//re mercirait mil fois de la bontè que vous aurez//pour mois sil vous plais, Mon maris// sapelle Ms bloquel benoit Couvreur//dèporte simple par ti sur le Calvados en nouvell// Calidonis, je vous en suplis engrase// (Z.10-13)

'ich danke Ihnen vielmals für die Güte, die Sie mir bitte entgegenbringen werden, mein Mann heißt Herr Bloquel Benoit, Dachdecker, einfacher Deportierter auf der Calvados nach NeuKaledonien, ich flehe Sie um Gnade an' [Abweichungen von der Orthographie wurden nicht wiedergegeben, S.M.]

Es fehlen also hier ein entsprechender Übergang sowie ein Expertenwissen bezüglich der Textstrukturierung. Zudem ist eine solche Strukturierung ungewöhnlich, da eine solch essentielle Nachricht hier sehr „spät“ formuliert wird. Das Verständnis bzw. die kommunikative 
Absicht bleiben trotzdem klar, da global betrachtet, die zentralen Informationen versprachlicht werden - selbst wenn dieser wichtige Inhalt gleichsam ,nachgereicht“" wird. ${ }^{26}$

Ansonsten ist der Brief weitgehend kohärent, was durch verschiedene Mittel, wie Rekurrenzen (z.B. wortwörtliche Wiederaufnahme: Maissieur 'Herr', Z. 4, 7); Verwendung von Pro-Formen (sa [Ref.: Mon maris] dernier lètre 'Sein [Ref: Mein Ehemann] letzter Brief', Z. 5f.; vous [Ref.: Maissieur] 'ihr' [Ref: Herr/Herren], Z. 4, 9, 10, 11, 15, 16), semantische Isotopien (z.B. Themenfeld des Bittens/Dankens: je vous re mericirait mil fois 'tausend Dank', Z.11; en vous remercient 'indem ich Ihnen danke', Z.11; sil vous plais 'bitte', Z.16f.; je vous en suplis 'Ich flehe Sie an', Z.15) oder auch die Verwendung von Konnektoren (z.B. argumentativ-kausaler Konnektor: Car 'Denn', Z.5, der hier die Rechtfertigung einleitet, weshalb ausgerechnet die Bitte der Autorin erhört werden soll und zugleich ein Marker für ein höheres Register zu sein scheint) gewährleistet ist.

\subsection{Diskurstraditionelle Ebene}

\subsubsection{Textaufbau: Makrostruktur}

Ein exemplarischer Blick auf die Makrostruktur wird nun - und dies sei vorweggenommen zeigen, dass sich die ungeübte Schreiberin auch auf dieser Ebene weitgehend auf den klassischen, d.h. an die ars dictaminis angelehnten Aufbau (vgl. oben), bezieht und diesen als Schablone nimmt - die Reihenfolge der traditionellen Bestandteile wird allerdings etwas abgeändert. Die salutatio (Z. 2-3) besitzt mit Ministère de la Marine 'Marineministerium' (Z. 2), Maissieur 'Mein Herr/Meine Herren' (Z. 4), eine zweiteilige Struktur, wobei offen bleiben muss, ob der erste Teil tatsächlich als Teil dieser Grußformel gedacht ist. Ein explizites exordium, das den Empfänger - ganz im Sinne einer captatio benevolentiae - zu Wort kommen ließe, ist nicht vorhanden, vielmehr geht die Autorin gleich in medias res, da die petitio, die hier ohne die erwartbare Überfrachtung mit positiven politeness-Strategien (vgl. Held 2005: 53) auskommt, als eigentliche Sprechhandlung (Maissieur si vous èt tès àseé bon pour me donner des nouvelle de mon maris 'Mein Herr/Meine Herren, wenn Sie so gut wären, mir Neuigkeiten bezüglich meines Ehemannes zu geben', Z.4f.) folgt. Diese Bitte wird aber - und dies entspricht nicht der klassischen Reihenfolge - später wiederholt, wenn es heißt je vous en suplis engrase de bien//vou loire me donné de ses nouvelle, silvous plais 'ich flehe Sie um Gnade, mir Neuigkeiten von ihm zu geben, bitte' (Z.15). Der Umstand, dass sich diese letzte Supplik am Ende des Briefs befindet, verleiht dem Gesagten noch einmal einen gewissen Nachdruck. Die Wiederholung kann damit - entgegen bisheriger Einschätzungen (vgl. Spitzer 1921/1976: 285) - als eine Argumentationsstrategie gesehen werden, durch die der Sprechakt zusätzlich untermauert wird.

Auf die erste petitio folgt eine Art narratio als illokutionsstützende Phase, in der die Autorin erklärt, weshalb die Bitte erfüllt werden soll (vgl. Z.5-8). Mit dem performativen Verb terminer 'aufhören' am Ende des Briefs wird eindeutig eine Schlussformel in der conclusio gesetzt (je termine en vous remercient, je vous salut femme bloquel 'ich beende [den Brief]

26 Ein Grund hierfür liegt darin, dass Experten - im Gegensatz zu Novizen - wesentlich mehr Planungszeit verwenden und ihre Texte meist vorab sehr detailliert organisieren und strukturieren. Nachdem Experten die Planung beendet haben, unterbrechen sie ihren Schreibprozess kaum. Die Schreibnovizen hingegen beginnen relativ spontan mit dem Verfassen des Texts, doch wird hier der Prozess nach jedem kohärenten Absatz unterbrochen, um den neuen Absatz zu planen. So sind Experten beim Schreiben wesentlich schneller und können so komplexere und umfangreichere Texte in weniger Zeit produzieren (vgl. Sasaki 2000: 279f.). 
indem ich Ihnen danke, ich grüße Sie Frau Bloquel' (Z.19f.), die allerdings ohne die in diesen Briefen üblichen Unterwerfungsriten der ALTER-Erniedrigung auskommt.

\subsubsection{Formelhaftigkeit vs. „freier Teil“6}

Die Untersuchung des Textaufbaus des vorliegenden Briefs hat eine starke imitatio der gängigen, zur damaligen Zeit bekannten Briefsteller und - damit verbunden - der ars dictaminis illustriert; stets hält sie sich dabei auch an bestimmte vorgegebene, epochenspezifische Floskeln der Höflichkeit. Obwohl es dabei zu einer gewissen Redundanz kommt, beachtet die Schreiberin - der Diskurstradition 'Bitte' entsprechend - eine gewisse Dekorumspflicht, indem die Verfasserin immer wieder, textstrukturell sinnvoll, bestimmte Floskeln einfügt (vgl. z.B. je vous//re mercirait mil fois de la bontè que vous aurez pour mois 'ich bedanke mich tausendfach bei Ihnen für die Güte, die Sie mir erweisen werden', Z. 10f.). Diese sind nicht nur textgliedernd, sondern bezwecken meist auch eine Erhöhung des Adressaten bzw. damit einhergehend, eine Unterwerfung des Schreibers (vgl. Maissieur 'Herr', Z. 4, 7; je vous salut 'ich grüße Sie', Z. 20). Betrachtet man die Floskel der beiden Bitten, si vous pouviez estre àsè bont èt est mable 'Wenn Sie so gut liebenswert wären' (Z.10) sowie je vous en suplis engrase de bien//vou loire me donné de ses nouvelle 'Ich bitte Sie um Gnade, mir seine Neuigkeiten zu überbringen' (Z.15f.), so zeigt dies, dass hier offene Bittformeln verwendet werden, in denen das Gesuch direkt formuliert wird.

Trotz dieser ritualisierten Formeln kommt es im „freien“ Teil der narratio zu einem (scheinbaren) Bruch mit der Diskurstradition des offiziellen Bittbriefs, da sich die Rechtfertigung des Schreibanlasses nicht in die epochenspezifische Formelhaftigkeit fügt und sie außerdem - durch das Anführen der Information über den schlechten Gesundheitszustand ihres Mannes (quil mècrié quil ést taità laupitale maide 'den er mir schreib, dass er im Krankenhaus sei; krank', Z.7) - etwas zu Persönliches für eine solch formelle Supplik anführt. Doch gerade dieser „Ausbruch“ aus den gängigen Traditionen und Mustern könnte der Autorin besonderes Gehör verschaffen, da sie dadurch auch auf emotionaler Ebene eine Legitimation ihres Anliegens liefert.

\section{Fazit}

Die Analyse zeigte ein interessantes Schwanken zwischen colto und semicolto bzw. incolto. Die Tatsache, dass es sich um eine unroutinierte Schreiberin handelt, erkennt man insbesondere an den hier herausgestellten, vor allem (ortho-)graphischen Besonderheiten, die ganz typisch für solche Texte sind, wie z.B. der normfernen Verschriftung von Lauten oder dem Fehlen textstrukturierender Absätze.

Und dennoch ist sich die Verfasserin des Briefs beispielsweise darüber bewusst, dass bei einer solchen Art von Text Anrede, Schlussformel und ein Mittelteil mit dem eigentlichen Gesuch benötigt werden, die hier, wenn auch abgeändert, an die Briefsteller bzw. die ars dictaminis angelehnt sind und zugleich bestimmte epochenspezifische Höflichkeitsfloskeln beinhalten. Daraus folgt, dass sie nicht nur über ein bestimmtes, allgemeines Wissen über den Sprechakt 'Bitte' verfügt, sondern tatsächlich auch eine Grundkenntnis von der Diskurstradition 'Bittbrief' besitzt.

Der hier analysierte Brief weist auch weitere Elemente auf, die durchaus von einer gewissen Erfahrung mit der Textkultur zeugen, und alle wichtigen Anliegen wurden treffend 
übermittelt. Die Bitte wurde vorgebracht und verstanden, was insbesondere durch die Randbemerkung, die Dame solle im Ministerium vorstellig werden, zum Ausdruck kommt. Die „Defizite“ auf der Mikroebene, so die Schlussfolgerung, können auf der übergeordneten Makroebene ausgeglichen werden, sodass gezeigt werden konnte, dass die semicolti durchaus erfolgreiche Kommunikationsstrategien anwenden. Es ist also ein Umdenken im Sinne einer lösungsorientierten, positiven Perspektive bzgl. des Schriftguts solcher semicolti vonnöten. Denn die Nichteinhaltung gewisser Verschriftlichungsparameter muss ein erfolgreiches Schreiben nicht verhindern.

\section{Literatur}

Barbaçon, L.-J. (2006): Chronologie relative à la déportation, transportation et relégation française. In: Les bagnes coloniaux, Instruments de recherche (Criminocorpus online, mis en ligne le 01 janvier 2006, http://criminocorpus.revues.org/142, letzter Zugriff 10.07.2016).

Bax, M. (1991): Historische Pragmatik: Eine Herausforderung für die Zukunft. In: Busse, D. (Hg.), Diachrone Semantik und Pragmatik. Tübingen: Niemeyer. 197-213.

Benevolo, L. (1979): La laurea dell'obbligo. Roma \& Bari: Laterza.

Berruto, G. (1983): L'italiano popolare e la semplificazione linguistica. Vox Romanica 42. 38-79.

Branca-Rosoff, S. \& Schneider, N. (1994): L'écriture des citoyens. Une analyse linguistique de l'écriture des peu-lettrés pendant la période révolutionnaire. Paris: Klincksieck.

Brown, P. \& Levinson, S. (1978/1987): Politeness. Some Universals in Language Usage. Cambridge: University Press.

Bruni, F. (1984): L'italiano. Elementi di storia della lingua e della cultura. Torino: UTET.

Catach, N. (1996): Dictionnaire historique de l'orthographe française. Paris: Larousse.

D’Achille, P. (1994): L'italiano dei semicolti. In: Serianni, L. \& Trifone, P. (Hg.), Storia della lingua italiana. Band 2: Scritto e parlato. Torino: Einaudi. 41-79.

De Mauro, T. (1970): Per lo studio dell'italiano popolare unitario. In: Rossi, A. (Hg.), Lettere da una tarantata. Bari: De Donato. 43-75.

Dion, I. \& Taillemite, H. (2007): Lettres du bagne. Aix en Provence: Collection d'outre-mer.

Droysen, J. G. (1977): Historik. Rekonstruktion der ersten vollständigen Fassung der Vorlesungen (1857). Grundriß der Historik in der ersten handschriftlichen (1857/1858) und in der letzten gedruckten Fassung, historisch-kritische Ausgabe von Peter Leyh. Band 1. Stuttgart (Bad Cannstatt): Frommann-Holzboog.

Ernst, G. (1999): Zwischen Alphabetisierung und 'français populaire écrit'. Zur Graphie privater französischer Texte. In: Ammon, U., Mattheier K. J. \& Nelde, P. H. (Hg.), Historische Soziolinguistik. Tübingen: Niemeyer (= Sociolinguistica. Internationales Jahrbuch für europäische Soziolinguistik 13). 91-111.

Fabre, D. (Hg.) (1993): Ecritures ordinaires, Paris: P.O.I. \& Centre Georges Pompidou.

Fesenmeier, L. (2005): Justizielle Texte aus Prato. Ein Fall für ganzheitliche Textbetrachtung. In: Schrott, A. \& Völker, H. (Hg.), Historische Pragmatik und historische Varietätenlinguistik in den romanischen Sprachen. Göttingen: Universitätsverlag. 157- 170.

Fleischman, S. (1990): Philology, Linguistics, and the Discourse of the Medieval Text. Speculum 65. 19-37.

Frei, H. (1929): La grammaire des fautes. Genève: Slatkine.

Fresu, R. (2014): Scritture die semicolti. In: Antonelli, G., Motolese, M. \& Tomasin, L. (Hg.), Storia dell'italiano scritto. Vol. III: Italiano dell'uso. Roma: Carocci. 195-223.

Gleßgen, M.-D. (2005): Diskurstraditionen zwischen pragmatischen Vorgaben und sprachlichen Varietäten. Methodische Überlegungen zur historischen Korpuslinguistik. In: Schrott, A. \& Völker, H. (Hg.), Historische Pragmatik und historische Varietätenlinguistik in den romanischen Sprachen. Göttingen: Universitätsverlag. 207-228

Goffmann, E. ( $\left.{ }^{4} 2008\right)$ : Interaction Ritual: Essays in Face to Face Behavior. New Brunswick (NJ): Aldine Transaction [Original: 1967].

Große, S. (2003): Französische Briefsteller. In: Aschenberg, H. \& Wilhelm, R. (Hg.), Romanische Sprachgeschichte und Diskurstraditionen. Tübingen: Narr. 135-161.

Haferland, H. \& Ingwer, P. (1996): Eine Theorie der Höflichkeit. In: Haferland, H. \& Ingwer, P. (Hg.), Höflichkeit. Osnabrück: OBST (= Osnabrücker Beiträge zur Sprachtheorie). 527-569. 
Held G. (2005): Der Einfluss von Höflichkeit auf die mittelalterliche Briefkunst - exemplarische Überlegungen zur Entwicklung von Textstruktur und Syntax vom dictamen zur freien Briefpraxis. In: Schrott, A. \& Völker, H. (Hg.), Historische Pragmatik und historische Varietätenlinguistik in den romanischen Sprachen. Göttingen: Universitätsverlag. 45-61.

Held, G. (2006): Schwerpunkt der historischen Pragmalinguistik: exemplarische Fallstudien/Linguistique pragmatique: études de cas. In: Ernst, G. (Hg.), Romanische Sprachgeschichte/Histoire linguistique de la Romania. 2. Teilband. Berlin \& New York: de Gruyter (= Handbücher zur Sprach- und Kommunikationswissenschaft 23.2). 2302-2318.

Held, G. (2008): Höflichkeit. In: Kolboom, I., Kotschi, K. \& Reichel, E. (Hg.), Handbuch Französisch. Sprache. Literatur. Kultur. Gesellschaft. Für Studium, Lehre, Praxis. Berlin: Schmitt. 357-363.

Leech, G. N. (1982): Principles of Pragmatics. London: Longman.

Lyons, M. (2013): The Writing Culture of Ordinary People in Europe, c. 1860-1920. Cambridge: Cambridge University Press.

Koch, P. \& Oesterreicher, W. (22011): Gesprochene Sprache in der Romania. Französisch, Italienisch, Spanisch. Berlin, New York: de Gruyter. (= Romanistische Arbeitshefte 31).

Koch, P. \& Oesterreicher, W. (2001): Gesprochene Sprache und geschriebene Sprache/ Langage parlé et langage écrit. In: Holtus, G., Metzeltin, M. \& Schmitt, C. (Hg.), Lexikon der Romanistischen Linguistik. Band I/2. Tübingen: Niemeyer. 584-60.

Massariello Merzagora, G. (2008): La scrittura degli emigrati. In: Sala, R. \& Massariello Merzagora, G. (Hg.), Radio Colonia. Emigrati italiani in Germania scrivono alla radio. Torino: UTF.

Oesterreicher, W. (1994): El español en textos escritos por semicultos. Competencia escrita de impronta oral en la historiografia indiana (s. XVI). In: Lüdtke, J. (Hg.), El español de America en el siglo XVI, Actas del Simposio del Instituto Ibero-Americano de Berlin, 23 y 24 abril de 1992. Frankfurt a. M., Madrid: Vervuert/Iberoamericana (= Bibliotheca Ibero-Americana 48). 155-190.

Oesterreicher, W. (1998): Nähesprachlich geprägtes Schreiben in der Kolonialhistoriographie Hispanoamerikas. (1500-1615). In: Raible, W. (Hg.), Medienwechsel: Erträge aus zwölf Jahren Forschung zum Thema 'Mündlichkeit und Schriftlichkeit'. Tübingen: Narr (= ScriptOralia 53). 76-78.

Oesterreicher, W. (2005): Autonomisation du texte et recontextualisation. Deux problèmes des sciences du texte. In: Murgía, A. (Hg.), Sinn und Referenz. Festschrift für Georges Kleiber/Sens et Références. Mélanges pour Georges Kleiber. Tübingen: Narr. 199-222.

Prein, A. (1921): Syntaktisches aus französischen Soldatenbriefen. Gießen: Selbstverlag des romanischen Seminars.

Raible, W. (1995): Kulturelle Perspektiven auf Schrift und Schreibprozesse. In: Raible, W. (Hg), Kulturelle Perspektiven auf Schrift und Schreibprozesse. Elf Aufsätze zum Thema Mündlichkeit uns Schriftlichkeit. Tübingen: Narr. vii-xxii.

Sasaki, M. (2000): Toward an Empirical Model of EFL Writing Processes. An Exploratory Study. Journal of Second Language Writing 9/3. 259-291.

Schrott, A. (2015): Präsente Schreiber(innen): Nähe und Lebendigkeit in privaten Briefen aus diskurstraditioneller Sicht. In: Bernsen, M., Eggert, E. \& Schrott, A. (Hg.), Historische Sprachwissenschaft als philologische Kulturwissenschaft. Festschrift für Franz Lebsanft zum 60. Geburtstag. Bonn: Vandenhoeck \& Ruprecht/Bonn: University Press. 479-497.

Spitzer, L. (1976): Lettere di prigionieri di guerra italiani, 1915-1918. Torino: Einaudi [Original: Italienische Kriegsgefangenenbriefe. Bonn: Hanstein 1921].

Stark, E. (2008): Einzelaspekt: Wortstellung und Informationsstruktur. In: Kolboom, I., Kotschi, T. \& Reichel, E. (Hg.), Handbuch Französisch. Sprache. Literatur. Kultur. Gesellschaft. Für Studium, Lehre, Praxis. Berlin: Schmitt. 311-317.

Stoll, E. (1997): Konquistadoren als Historiographen. Diskurstraditionelle und textpragmatische Aspekte in Texten von Francisco de Jerez, Diego de Trujillo, Pedro Pizzaro und Alonso Borregán. Tübingen: Narr.

Testa, E. (2014): L'italiano nascosto. Una storia linguistica e culturale. Torino: Einaudi.

Thompson, E. P. (1966): History from Below. Times Literary Supplement 7 (April). 279-80.

Thun, H. (2011): Die diachrone Erforschung der français régionaux auf der Grundlage des Corpus Historique du Substandard Français. In: Schlaak, C. \& Busse, L. (Hg.), Sprachkontakte, Sprachvariation und Sprachwandel. Festschrift für Thomas Stehl zum 60. Geburtstag. Tübingen: Narr. 359-394.

\section{Quellen}

Anonymous (1813): Le Secrétaire de la cour impériale de France. Sixième édition. Paris: Barba. 
Bernier, J. (1829): Code épistolaire, contenant les règles, les principes et le cérémonial du style épistolaire, avec de modèles de lettres sur toute espèce de sujets. Bruxelles: De Mat.

ANOM: FM H 70-76, Fonds ministériels (FM)

ANOM: FM H 248-262, Fonds ministériels (FM)

ANOM: FM H 416 und H 698, Fonds ministériels (FM) 


\section{Anhang}

Abbildung 1: Brief „Bloquel““ ANOM FM H72

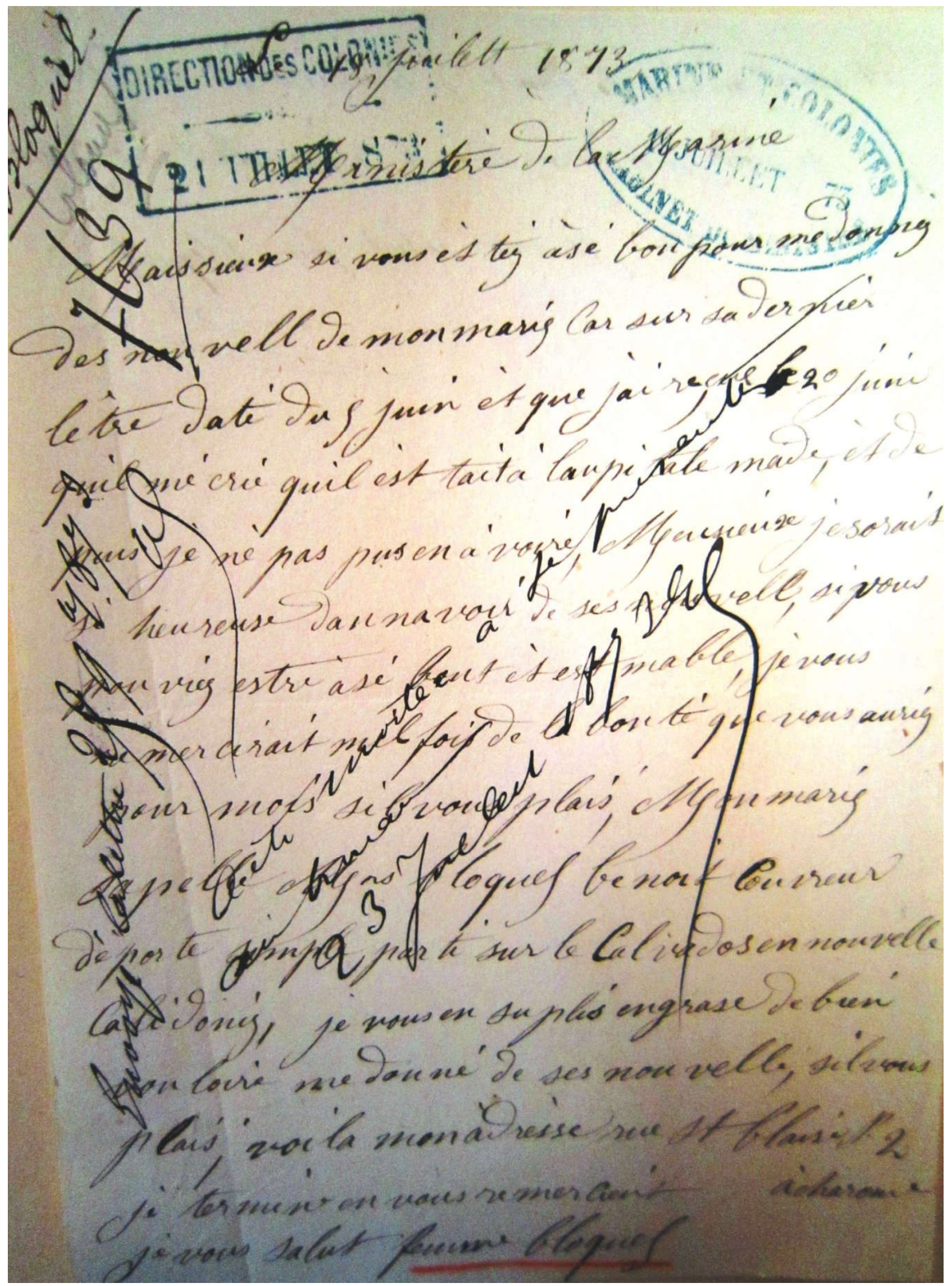


Transkription 1: Brief „Bloquel“ ANOM FM H72

Le 18 juilett 1873

Ministère de la Marine

Maissieur si vous èt tèz àsé bon pour me donnez des nouvell de mon maris car sur sa dernier lètre daté du 5 juin èt que jai reçue le 20 juin quil mécrié quil ést taità laupitale made, èt de puis je nè pas pus en à voire, Maissieur jeserait si heureuse dannavoir de ses nouvelle, si vous pouviez estre àsè bont èt est mable, je vous re mercirait mil fois de la bontè que vous auréz pour mois sil vous plais, Mon maris sapelle Ms bloquel benoit couvreur dèporte simple par ti sur le Calvados en nouvelle Calidonis, je vous en suplis engrase de bien vou loire me donné de ses nouvelle, silvous plais, voila monadrèsse rue st blaisé $\mathrm{N} 2$

àMaronne

je termine en vous remercient je vous salut femme bloquel 


\title{
Die Verwendung der perfektiven Präsensformen im Russischen, Slovenischen und heutigen Resianischen
}

\author{
Malinka Pila (Konstanz)
}

\begin{abstract}
My paper concerns the use of perfective present forms in Russian, Slovene and Resian, a Slovene dialect in a setting of absolute language contact with Italian. While in Russian the use of perfective present forms is very limited, in Slovene there are no fundamental restrictions for perfective forms to be used in the present tense, apart from, of course, the expression of ongoing processes. In the Slovene present tense, the perfective aspect can have habitual/iterative and potential meanings; it is often used in performative utterances, within the context of the historical present and in the so-called gnomic present.

Theoretically, all these uses of perfective present forms are permitted in Resian, too. Nevertheless, it seems that in the present tense, Resian generally opts for the imperfective aspect. The present paper discusses the hypothesis of this phenomenon being related to the Resian situation of absolute contact with Italian.
\end{abstract}

\section{Einleitung}

In meinem Beitrag möchte ich den Gebrauch der perfektiven Präsensformen im Russischen, Slovenischen und modernen Resianischen vergleichend darstellen. Das Resianische ist eine Mikrosprache auf slovenischer Dialektbasis, die in der Hauptsache im Resiatal (Val Resia) im Nordosten Italiens, an der Grenze zu Slovenien, gesprochen wird. Dabei handelt es sich im Prinzip um vier Dörfer mit jeweils einer eigenen Dialektvarietät: San Giorgio, Gniva, Oseacco und Stolvizza. Daneben bestehen in diesem Tal noch einige weitere Dörfer mit Mischvarieteten. Das Resianische befindet sich in einer Situation des „totalen“ Sprachkontakts mit dem Italienischen, d.h. alle Sprecher des Resianischen beherrschen auch das Italienische, das die Rolle einer Dachsprache für das Resianische spielt (fremdes Dach), während der Kontakt mit dem Slovenischen als der genetisch am nächsten stehenden Standardsprache deutlich geringer ist und in den vergangenen Jahrhunderten überhaupt keine Rolle spielte. ${ }^{1}$

Über den Aspektgebrauch im Präsens liegen schon einige vergleichende Arbeiten zum Russischen und Slovenischen vor, zum Beispiel die Dissertation von O.S. Plotnikova aus den Siebziger Jahren, die aber nie publiziert wurde (Plotnikova 1975), die Aufsätze von A. Derganc (Derganc 1992-1993, 2002, 2003, 2010), ein Artikel von B. Wiemer (Wiemer 2014), in dem alle slavischen Sprachen in Bezug auf die Koinzidenzfälle analysiert werden, die Monographie von S. Dickey (Dickey 2000), der den Aspektgebrauch in den slavischen Sprachen auf allen Zeitstufen analysiert, sowie auch die Dissertation der Autorin dieses Beitrags (Pila

1 „Totaler (oder absoluter) Sprachkontakt“ liegt nach Breu (2011: 431) vor. Überlegungen über die geographische Lage, den Ursprung und die Entwicklung des Resianischen und der wie das Resianische in Julisch-Venetien gesprochenen slovenisch-basierten Minderheitendialekte von Torre und Natisone finden sich in Steenwijk (1992: 1-3) und Benacchio (2002: 17-20, 63-70). 
2013), in der die Funktionen des pf. (perfektiven) und des ipf. (imperfektiven) Aspekts im Russischen und Slovenischen unter Einschluss des Präsens beschrieben werden.

Zwar finden sich einige interessante Ansätze zur Beschreibung des resianischen Verbalaspekts auch in der Grammatik von H. Steenwijk (Steenwijk 1992), aber eine systematische Analyse des Gebrauchs und der Bedeutungen des pf. und ipf. Aspekts steht noch aus. Interessant ist das resianische Aspektsystem nicht zuletzt deshalb, weil diese Mikrosprache im absoluten Kontakt mit einer romanischen Standardsprache steht, die die Aspektkategorie nicht, wie für die slavischen Sprachen typisch, durch Derivation ausdrückt, sondern über eine progressive Periphrase und - auf das Präteritum beschränkt - durch die flexivisch-analytische Opposition von Imperfekt und Perfekt. Hinzu kommt der Jahrhunderte währende Kontakt mit dem ebenfalls romanischen Friaulischen.

Meine laufenden Arbeiten zum resianischen Aspektsystem sind auch als Beitrag zur Beschreibung der aspektuellen Variation in den slavischen Sprachen insgesamt zu verstehen. Eine zentrale Rolle spielt hierbei die Frage, ob und wie die Situation des totalen Sprachkontakts mit dem Italienischen einerseits und die Isolierung vom Standardslovenischen (und den slovenischen Dialekten) andererseits das Aspektsystem des Resianischen beeinflusst haben. Der hier vorliegende Beitrag ist dem Präsens gewidmet, weil hier die auffälligsten Unterschiede zwischen dem Russischen und dem Slovenischen zu beobachten sind.

Hinsichtlich des Russischen und Slovenischen beziehe ich mich hauptsächlich auf das Datenkorpus, das ich für meine Dissertation analysiert habe. Es handelt sich um literarische Werke des 20. Jahrhunderts: Pomladni dan von C. Kosmač (slovenisches Original und russische Übersetzung), Čemodan von S. Dovlatov (russisches Original und slovenische Übersetzung) sowie die slovenische und russische Übersetzung des Romans Il deserto dei Tartari des italienischen Autors D. Buzzati.

Das resianische Korpus für meine Analyse besteht größtenteils aus den Materialien, die ich bei meiner Feldforschung im Resiatal in den Jahren 2015-2016 gesammelt habe. Es handelt sich um transkribierte Aufnahmen von Interviews sowie mündlichen Übersetzungen vorgegebener italienischer Sätze ins Resianische. ${ }^{2}$ Daneben entnehme ich einige Beispiele aus Publikationen anderer Autoren sowie aus Informationsbroschüren für Touristen. In den letzteren Fällen wird jeweils die Orthographie der Vorlage verwendet, während die Transkription des von mir selbst gesammelten Materials den orthographischen Regeln nach Steenwijk (1994) folgt, und zwar jeweils in Abhängigkeit von der Varietät des Resianischen, aus der das gewählte Beispiel stammt.

Nach diesen notwendigen Vorbemerkungen komme ich nun zu den ersten Ergebnissen meiner Analyse. Ich gehe dabei von den Verhältnissen im Russischen aus und stelle ihnen den Aspektgebrauch im Slovenischen und Resianischen gegenüber. Abschließend wird dann der Gebrauch des Verbalaspekts im Resianischen mit demjenigen in anderen slavischen Sprachen, die sich in Situationen von totalem Sprachkontakt befinden, verglichen.

\section{Der Aspektgebrauch im Präsens des Russischen}

Bekanntlich ist das Russische unter den slavischen Sprachen diejenige, welche die stärksten Beschränkungen für den Gebrauch der perfektiven Verbformen aufweist. So wird zum Beispiel auf der Stufe der Gegenwart die aspektuelle Opposition fast völlig neutralisiert, da der pf. Aspekt nur in ganz speziellen Fällen benutzt werden kann. Abgesehen vom aktuellen Prä-

2 Beispiele ohne weitere Quellenverweise stammen aus diesen Materialien. 
sens ist der pf. Aspekt beispielsweise auch vom direkten Ausdruck der Habitualität und des historischen Präsens ausgeschlossen. Möglich ist der pf. Aspekt in den beiden folgenden Fällen: nagljadno-primernoe značenie (= anschaulich-exemplarische Bedeutung), d.h. die (stilistisch markierte) Einbettung wiederholter Handlungen, (1)-(2), und potencial'noe značenie (= potenzielle Bedeutung), d.h. der Ausdruck der Möglichkeit / Unmöglichkeit, eine Handlung zu verwirklichen, (3)-(4). ${ }^{3}$

А у вас всегда зуд? - Всегда. То есть вот как сяду ${ }^{\mathrm{pf}}$, так все и засвербит ${ }^{\mathrm{pf}}$. (Kosmač 1988: 75)

'Haben Sie immer Juckreiz? - Immer. Das heißt, sobald ich mich setze, juckt es mich sofort.'

А помнишь, как он, бывало, по воскресеньям оденется ${ }^{\text {pf }}$ и ходит ${ }^{\text {ipf }}$ по комнате, выпрямившись, как струна! Повернется ${ }^{\text {pf }}$ на каблуках, поведет ${ }^{\text {pf }}$ плечами и оглядит ${ }^{\mathrm{pf}}$ себя. (Kosmač 1988: 25)

'Erinnerst du dich, wie es vorkam, dass er sich an Sonntagen ankleidet und im Zimmer aufrecht und pfeilgerade herumläuft! Er dreht sich (dann) auf seinen Absätzen, zuckt mit den Achseln und blickt an sich herab.'

(3) Отец поднял глаза к небу - видать, этого человека ничем не прошибешь ${ }^{\mathrm{pf}}$. (Kosmač 1988: 51)

'Der Vater schaute zum Himmel - offensichtlich kannst du diesen Menschen mit nichts durchdringen. (wörtlich: „offensichtlich durchdringst du diesen Menschen mit nichts")'

(4) Конечно, теперь он офицер, [...] а все-таки - чувствовал Джованни Дрого лучшие годы, годы ранней юности, уже не вернешь ${ }^{\text {pf }}$. (Buccati 2010: 6)

'Freilich! Er ist jetzt ein Offizier, [...] jedoch spürte Giovanni Drogo, dass die besten Jahre, die frühen Jugendjahre, nicht mehr zurückgeholt werden können. (wörtlich: ,dass du sie nicht mehr zurückholst" ).'

Wie diese Beispiele zeigen, muss im Russischen ein spezieller Kontext vorliegen, der den Gebrauch des pf. Aspekts in solchen Fällen erlaubt. Im Falle der anschaulich-exemplarischen Bedeutung, (1)-(2), muss die Iterativität übergeordnet ausgedrückt sein, wie zum Beispiel über die Verbindung des Adverbs всегда 'immer' mit der Konstruktion вот как... так все... 'sobald... dann sofort...' in (1) oder бывало 'es pflegte zu sein' in (2). Wenn kein solcher übergeordneter Hinweis auf die Iterativität vorliegt, wird die Handlung als Einzelhandlung oder Handlungskette interpretiert. ${ }^{4}$ Im Falle der potentiellen Bedeutung wie in den Beispielen

3 Für Aufzählungen und Beschreibungen der Gesamtheit der Funktionen des pf. Aspekts im Russischen, einschließlich der ,anschaulich-exemplarischen“ und der „potenziellen Bedeutung“, vgl. zum Beispiel Bondarko (1971: 22-24), Zaliznjak \& Šmelev (2000: 19-21) und Petruchina (2009: 64-66).

$4 \mathrm{Zu}$ beachten ist, dass sich die Iteration sowohl auf die Gegenwart, vgl. (1), wie auch auf die Vergangenheit, vgl. (2), beziehen kann. In beiden Fällen stehen die Verben im pf. Präsens. Auf der Vergangenheitsstufe benutzt das Russische den pf. Aspekt nur im Falle der summarischen Iteration, das heißt etwa bei Adverbien wie "zweimal”, “dreimal” etc. (Barentsen 1992, 1994). 
(3)-(4) hat das Verb eine modale Nuance, der Kontext ist in der Regel allgemein gehalten (vgl. hier das ,allgemeine $d u^{\text {" }}$ ) und oft liegt Negation vor. ${ }^{5}$

Der pf. Aspekt im Russischen wird auch mit performativen Verben im Falle der Koinzidenz verwendet, d.h. wenn der Sprechakt und die Realisierung der Handlung zusammenfallen, vgl. etwa die Beispiele (5)-(7) aus Apresjan (1986: 211, 215). Trotzdem ist der Gebrauch des pf. Aspekts im Falle der Koinzidenz insgesamt selten und es wird normalerweise im Russischen der ipf. Aspekt benutzt (Derganc 2003: 74).

\section{Покаюсь $^{\mathrm{pf}} /$ признаюсь ${ }^{\mathrm{pf}} /$ сознаюсь $^{\mathrm{pf}}$ : это сделал я.}

'Ich werde büßen/ ich gebe zu/ ich gestehe: das habe ich getan.'

$$
\begin{aligned}
& \text { Пожелаю }{ }^{\mathrm{pf}} \text { Вам удачи } \\
& \text { 'Ich wünsche Ihnen Erfolg.' }
\end{aligned}
$$

$$
\begin{aligned}
& \text { Попрошу }{ }^{\mathrm{pf}} \text { вас выйти } \\
& \text { 'Ich bitte euch hinauszugehen.' }
\end{aligned}
$$

Der pf. Aspekt kommt im Präsens des Russischen häufig in Aphorismen vor, das heißt im atemporalen gnomischen Präsens. Nach Petruchina (2009: 132) wird der pf. Aspekt hier häufig als die Möglichkeit (potencial'naja vozmožnost'), die Handlung zu verwirklichen, interpretiert. $^{6}$

$$
\text { Имеем - не храним, потеряем }{ }^{\mathrm{pf}}-\text { плачем. }
$$

'Was wir haben, bewahren wir nicht. Wenn wir es verlieren, dann weinen wir ihm nach.'

$$
\begin{aligned}
& \text { Кто посеет }{ }^{\mathrm{pf}} \text { ветер, пожнет }{ }^{\mathrm{pf}} \text { бурю. } \\
& \text { 'Wer Wind sät, wird Sturm ernten.' }
\end{aligned}
$$

$$
\begin{aligned}
& \text { Не обманешь }{ }^{\text {pf }} \text { - не продашь }{ }^{\text {pf }} \text {. } \\
& \text { 'Wenn du nicht betrügst, verkaufst du nicht.' }
\end{aligned}
$$

Zusammenfassend können wir in Bezug auf das Russische sagen, dass die Verwendung des pf. Aspekt sehr begrenzt ist. Abgesehen vom Fall der Koinzidenz wird er nur bei atemporaler Gegenwart benutzt, und zwar, wenn die anschaulich-exemplarische oder die potentielle Bedeutung vorliegt, sowie auch im gnomischen Präsens.

Die Verhältnisse im Slovenischen und Resianischen sind komplizierter und vielfältiger. Zum Teil ist das auf die Unterschiede im Tempussystem im Vergleich mit dem Russischen zurückzuführen. Das Aspekt-Tempus-System des Russischen verfügt ja morphologisch über eine perfektive Präsensform, die aber Zukunftsbedeutung aufweist. Dagegen zeigt das Aspekt-Tempus-System des Slovenischen eine perfektive Präsensform, die gewöhnlich eine atemporale Bedeutung hat und nur selten eine Handlung in der Zukunft ausdrückt (Derganc 2003: 67). Die Zukunft wird im Slovenischen durch analytische Formen ausgedrückt, und zwar nicht nur im ipf. Aspekt wie im Russischen, sondern auch im pf. Aspekt. Eine ähnliche

\footnotetext{
5 Für die Beschreibung der Faktoren, die die Verwendung des pf. Aspekts mit ,,anschaulich-exemplarischer“ Bedeutung begünstigen, vgl. Bondarko (1971: 23) und Zaliznjak \& Šmelev (2000: 19). Hinsichtlich der ,,potentiellen Bedeutung“vgl. Bondarko (1971: 23) und Glovinskaja (2001: 68).

6 Diese Beispiele stammen aus dem Internet. Für weitere Beispiele vgl. Zimin (2008).
} 
Situation findet man im Resianischen, wobei allerdings neben dem Auxiliar 'sein' (bet) wie im Russischen und Slovenischen zusätzlich auch das Auxiliar 'wollen' (tët) vorkommt.

\section{Der Aspektgebrauch im Slovenischen und Resianischen}

\subsection{Iterativer und habitueller Gebrauch}

Das Slovenische ist die slavische Standardsprache, in der die pf. Verbformen wohl am häufigsten auf der Zeitstufe der Gegenwart außerhalb aktueller Handlungen, bei denen der ipf. Aspekt auch im Slovenischen obligatorisch ist, verwendet werden (Plotnikova 1998). Prinzipielle Beschränkungen bestehen überhaupt nicht. So ist der pf. Aspekt stilistisch neutral neben dem ipf. Aspekt etwa bei habituellen oder iterativen Handlungen möglich (Plotnikova 1975: 112, 1999: 75):

Spomeniki se rojevajo ${ }^{\text {ipf }}$ takole. Kipar zmodelira ${ }^{\text {pf }}$ glinast osnutek. Kalupar ga odlije $^{\text {pf }}$ v mavec. (Dovlatov 2006: 154)

'Denkmäler entstehen in folgender Weise: Der Bildhauer modelliert ein Model aus Ton. Der Formenmacher gießt es in Gips.'

Pomislili so: bolje je torej, če pozna geslo samo častnik. Toda zdaj zapustijo ${ }^{\text {pf }}$ Trdnjavo tri četrt ure pred menjavo straž... (Buzzati 2004: 34)

'Sie haben gedacht: es ist besser, wenn nur der Offizier das Kennwort weiß. So gehen sie jetzt (immer) 45 Minuten vor der Wachablösung hinaus...'

Toda taka drevesa navadno požagajo ${ }^{\text {pf }}$, - je vztrajal pob. (Kosmač 2001: 115)

'Dann sägen sie diese Bäume normalerweise ab, - insistierte der Junge.'

Liegen wie in diesen Beispielen terminative Verben vor, so wird im Slovenischen die pf. der ipf. Form vorgezogen, wenn der Fokus auf dem Erreichen der inhärenten Grenze der Handlung liegt. Das geschieht vor allem in Handlungsketten, in denen jedes Ereignis abgeschlossen wird, bevor das nachfolgende beginnen kann. Im Russischen erscheint in diesen Fällen immer eine ipf. Form, weil diese Sprache die Aufmerksamkeit auf die Makroebene der Wiederholung lenkt, das heißt indem sie die Handlung oder Handlungskette als ein 'Kontinuum' darstellt, während im Slovenischen der Fokus auf der Mikroebene der Iteration liegt, auf den einzelnen wiederholten Handlung. ${ }^{7}$ Wie oben erwähnt, ist in solchen Fällen der pf. Aspekt nur im Fall der Konstruktionen möglich, die anschaulich-exemplarische Bedeutung haben. ${ }^{8}$

Eine zum Slovenischen analoge Situation findet man im Resianischen, wo ebenfalls im Bereich von Habitualität, (14), und Iteration, (15)-(16), der Gebrauch beider Aspekte erlaubt ist, wie die nachfolgenden Beispiele zeigen.

7 Für die Definition der Makro-/Mikroebenen der Wiederholung vgl. Stunová (1993: 25), die das Problem hinsichtlich des Tschechischen und Russischen aspektuellen Verhaltens analysiert. Vgl. auch Breu (2000: 4447), wo das Problem der Makro-/Mikroebenen angesprochen wird, einschließlich der nagljadno-primernoe značenie. Der Aufsatz enthält im Übrigen auch Aussagen zur Koinzidenz und zu diversen Typen von transponiertem Präsens (etwa dem historischen Präsens).

8 Für eine eingehende, vergleichende Analyse der Verwendung des Verbalaspekts im Russischen und Slovenischen in iterativen Kontexten vgl. Benacchio \& Pila (2015). 
(14) Ko se dujdë ${ }^{\mathrm{pf}}$ nu-na ta viliki must, sa ma sa zvyt ... a sinistra.

'Wenn man zu der großen Brücke kommt, muss man abbiegen... a sinistra (= nach links).'

(15) Wsaki din an $\mathbf{d a}^{\mathrm{pf}}$ no jabulko otroko. Pa wčera an jë mu dal no jabulko.

'Jeden Tag gibt er dem Kind einen Apfel. Auch gestern gab er ihm einen Apfel.'

(16) Wsaki viječ policjot paraća ${ }^{\text {pf }}$ mülto, napišê ${ }^{p f}$ mülto an wsaki viječ, policjot da ${ }^{\text {pf }}$ mülto ... da no mülto midio.

'Jedesmal bereitet der Polizist einen Strafzettel vor, schreibt einen Strafzettel und jedesmal gibt der Polizist den Strafzettel ... gibt einen Strafzettel dem Arzt.'

Auch wenn das Resianische hier im Kontext von wiederholten Handlungen die pf. Form verwenden kann, ist allerdings zu beachten, dass; dies nur in viel geringerem Umfang geschieht als im Slovenischen. Sehr häufig fällt die Wahl sogar im Fall von Handlungsketten wie in (18), die im Slovenischen bevorzugt mit dem pf. Aspekt gebraucht werden, auf die ipf. Form.

(17) Vïlažej an dujaja ${ }^{\text {inf }}$ wökul marča, sri marča, pyrve dni avrïla, ma to nï wsakë lëtu par.

'Der Frühling kommt gegen März, gegen Mitte März oder in den ersten Tagen des April, aber das ist nicht jedes Jahr gleich.'

(18) Strok sa ji sijë $\ddot{i p f}^{\text {pf }}$ ta-w jisiny, ko to jë otobarja, wyr otobarja. Sa ji sijë ${ }^{i p f}$, sa prikopawa $^{\text {ipf }}$ njïwo, sa diwa ${ }^{\text {ipf }}$ gnuj, sa sije ${ }^{\text {ipf }}$ strok, tadij se ga pukrïwa ${ }^{\text {ipf }}$ spet ziz gnujon za wöjo mraza, ano ko to jë wökul favrarja, zadnje dni favrarja, pyrve dni marča strok pučnüwa ${ }^{\text {ipf }}$ gnyt, tadij, ti mäš ga rizkryt.

'Knoblauch pflanzt man im Herbst, im Oktober, Ende Oktober. Ihn pflanzt man, man hackt das Feld um, man gibt Mist hinzu, man pflanzt Knoblauch, man bedeckt ihn mit Mist gegen die Kälte, und Mitte Februar, Ende Februar, Anfang März fängt der Knoblauch an zu wachsen. Dann musst du ihn aufdecken.'

(19) Wsaki din pojütrë an paraćawa ${ }^{\text {ipf }}$ dwa panïna za wotroke.

'Jeden Tag, am Morgen, macht er zwei Brötchen für die Kinder.'

\subsection{Die potenzielle Bedeutung}

Der pf. Aspekt kommt im Slovenischen auch mit potenzieller Bedeutung vor; vgl. hierzu Bajec (1971) und Plotnikova (1975) mit den folgenden Beispielen:

(20) Kdo vzdigne ${ }^{\text {pf }}$ ta kamen? (Bajec 1971: 239)

'Wer kann diesen Stein hochheben? (wörtl. 'Wer hebt diesen Stein hoch?')'

(21) Na koncu se vendar najde mož, ki te premodri ${ }^{\text {pf }}$. (Plotnikova 1975: 92)

'Am Ende findet sich immer ein Mann, der dich betrügen kann. (wörtl. 'der dich betrügt')' 
Jaz vsega tega ne prenesem ${ }^{\mathrm{pf}}$. (ibid.)

'Das alles kann ich nicht ertragen. (wörtl. 'Das alles ertrage ich nicht')'

Im Resianischen habe ich im Verlauf meiner Feldforschungen vor Ort kein einziges Beispiel einer pf. Präsensform gefunden, das die Möglichkeit/Unmöglichkeit, die Handlung auszuführen, ausgedrückt hätte. Es scheint, als bevorzuge das Resianische in diesen Fällen absolut den ipf. Aspekt, so wie in den Beispielen (23)-(24). Diese Hypothese ist aber erst noch mit einer größeren Zahl von Informanten zu verifizieren.

Du wzdüwa ${ }^{\text {ipf }}$ no tako valïko pënjć?

'Wer kann einen so großen Stein hochheben? (wörtl. 'Wer hebt einen so großen Stein hoch?')'

(24) Puslüšaj me, izdë w dulïni ti ni kupüwašs ${ }^{\text {ipf }}$ ne ïše za 100 mijarjuw ewruw!

'Hör zu! Hier im Tal kannst du kein Haus für 100 tausend Euro kaufen. (wörtl.

'Hier im Tal kaufst du kein Haus für 100 tausend Euro')'

\subsection{Aspekt und Koinzidenz}

Für den vorliegenden Beitrag habe ich auch Koinzidenzfälle analysiert, also Sätze, die performative Verben enthalten, bei denen der Sprechakt und die Realisierung der Handlung zusammenfallen.

Im Slovenischen ist der Gebrauch des pf. Aspekts auch in diesen Kontexten erlaubt, auch wenn es hier - wie Derganc (2003:74) ausführt - auf den konkreten Einzelfall ankommt. So erscheinen einige Verbformen, wie vdam se 'ich ergebe mich' und priznam 'ich gebe zu', bevorzugt im pf. Aspekt. Andere Verbformen, wie prosim 'ich bitte' und predlagam 'ich schlage vor', erscheinen üblicherweise im ipf. Aspekt (Derganc ibid.). In einigen Fällen, wie bei obljubljati/obljubiti 'versprechen' und prisegati/priseči 'schwören', scheint es, als bevorzuge das Slovenische bei offiziellen Anlässen die ipf. Form. Bei inoffiziellen Gelegenheiten wird dagegen die pf. Form verwendet (Derganc 1992-1993: 72). Hier zwei Beispiele für den pf. Aspekt bei Koinzidenz im Slovenischen aus Derganc (2003: 73), gekürzt:

Priznam $^{\text {pf: }}$ to sem naredil jaz.

'Ich gebe zu: das habe ich getan.'

Obljubim $^{\text {pf }}$, da ti bom pomagal.

'Ich verspreche, dir zu helfen.'

Im Resianischen scheint die Zahl der performativen Verben im Vergleich zum Russischen und Slovenischen an sich schon eher klein zu sein: zwar bestehen Entsprechungen zu 'sich bedanken' und 'versprechen' in Form des pf. zahwalit bzw. des Paares obaćawat/obaćät, daneben für 'warnen' das pf. Verb ovizät ${ }^{10}$, ursprünglich eine Entlehnung aus italienisch

9 Zu dem pf. Verb obaćät 'verprechen' bestehen zwei ipf. Entsprechungen, obaćawat und ubićüwat.

10 Die hier zitierten Infinitivformen sind in der standardisierten Form nach Steenwijk (1994) wiedergegeben. Die tatsächlich beobachteten Koinzidenzfälle beziehen sich auf die Varietät von Stolvizza mit den ipf. Präsensformen zawaljüwen und ovizawen. Die zugehörigen Infinitive in dieser wie auch in den anderen Varietäten können noch nicht belegt werden. 
avvisare. Aber bis jetzt wurden keine Entsprechungen für 'zugeben', 'anerkennen', 'verbieten' etc. gefunden.

Generell benutzt das Resianischen in Fällen der Koinzidenz fast immer das ipf. Verb, so wie in (27)-(28). Der einzige mir bekannte Fall einer pf. Koinzidenzform ist zawalin wie in Beispiel (29), wobei hier auch die ipf. Form möglich wäre.

$$
\begin{aligned}
& \text { Ti obaćawen }{ }^{\text {ipf }} \text { da parajan zütra. } \\
& \text { 'Ich verspreche dir morgen zu kommen.' }
\end{aligned}
$$

Ti ovizawen ${ }^{\text {ipf }}$, ći ti ni prïdëš, ćon se ribjet.

'Ich warne dich: wenn du nicht kommst, werde ich mich ärgern.'

$$
\text { Te zawalin }{ }^{\text {pf }} / \text { zawaljüwen }^{\text {ipf }} \text { karjë čas. }
$$

'Ich danke dir vielmals.'

\subsection{Das historische Präsens}

Mit Hilfe des historischen Präsens, also der Wiedergabe einer vergangenen Handlung durch Präsensformen, kann eine Erzählung „lebendiger“ gestaltet werden als bei der Verwendung des unmarkierten Präteritums. Während hier im Russischen eine vollständige Neutralisierung im ipf. Aspekt vorliegt, weist etwa Bondarko (1959: 49-51) darauf hin, dass im Slovenischen im Gegensatz zur Mehrzahl der slavischen Sprachen eine vollständige und kohärente Aspektopposition innerhalb des historischen Präsens besteht. Derganc (2003: 73) präzisiert demgegenüber, dass im Slovenischen bei der Übertragung eines präteritalen Textes ins historische Präsens der Aspektgebrauch unverändert bleibt, was bedeutet, dass die Aspektwahl im historischen Präsens denselben Regeln folgt wie im Präteritum. Vgl. hierzu die Beispiele (30) und (31) mit pf. historischem Präsens:

(30) Dovolj je bil en sam strel, navaden strel iz puške, da se je Trdnjava prebudila. Na stražarskem mestu, kjer se je zgodila nesreča, [...] je velik vrvež [...]. Zdaj se prikaže $^{\mathrm{ipf}}$ še major Matti [...]. »Pravim: je bil zadet v čelo? « reče ${ }^{\text {pf }}$ Matti, naveličan ponavljanja. Tronk dvigne ${ }^{\text {pf }}$ svetilko in od blizu osvetlip ${ }^{\text {pf }}$ Lazzarijev obraz. Zdaj tudi sam opazi ${ }^{\text {pf }}$ luknjico, podzavestno sproži ${ }^{\text {pf }}$ prst, kot da bi se je hotel dotakniti, in ga takoj zmedeno odmakne ${ }^{\mathrm{pf}}$. (Buzzati 2004: 81-83)

'Ein einfacher Gewehrschuss hatte genügt, um die ganze Festung aufzuscheuchen. [...] Bei der Wachmannschaft [...], wo das Unglück geschehen ist, herrscht großes Durcheinander. [...] Nun erscheint auch Major Matti [...]. „Ich sage: Wurde er an der Stirn getroffen?" sagt Matti, verärgert darüber, dass er sich wiederholen muß. Tronk hebt die Laterne, leuchtet voll in das Gesicht von Lazzari, sieht ebenfalls die Einkerbung und streckt instinktiv den Finger aus, um sie zu berühren. Er zieht ihn aber sofort verstört zurück.'

(31) $\quad » N e$, ne bom ga več videl ...« sem zamrmral in zaprl oči. A še isti hip sem ga zagledal. [...] - Stopi naprej - pravi oče [...]. Previdno se pomaknem ${ }^{\text {pf }}$ po stezi, nato se obrnem ${ }^{\text {pf }}$ in se spet oprem $^{\text {pf }}$ na sekiro. (Kosmač 2001: 67-68)

'Nein, ich werde ihn nicht mehr wiedersehen..." murrte ich und schloss die Augen. Und noch im selben Moment erblickte ich ihn. [...] - Geh vorbei - sagt Va- 
ter [...].Vorsichtig gehe ich auf dem Pfad los, dann drehe ich mich um und stütze mich wieder auf die Axt.'

Was das Resianische angeht, hat es beim gegenwärtigen Stand der Untersuchungen den Anschein, dass das historische Präsens in Erzählungen nicht besonders häufig verwendet wird. Während der Aufnahme mündlicher Texte gebrauchte kein einziger Sprecher diese Form spontan, und bei den verschiedenen Versuchen, ein Textstück von einer italienischen Vorlage ins historische Präsens zu übersetzen, setzten die Informanten die Erzählung häufig ins Präteritum um. Dass das historische Präsens dennoch auch in dieser Mikrosprache zulässig ist, geht beispielsweise aus einer Broschüre hervor, in der mit dem historischen Präsens die Entstehung einer Molkerei im resianischen Dorf San Giorgio beschrieben wird. ${ }^{11}$ Auch wenn es sich wahrscheinlich um die Übersetzung einer italienischen Vorlage handelt und nicht um einen authentischen resianischen Text, so ist es doch bezeichnend, dass pf. Formen hier vollständig fehlen.

Ich gebe im Folgenden einen Ausschnitt aus diesem Text (in Originalorthographie) wieder. Es geht dabei um den Umbau eines Hauses zu einer Molkerei, weil die alte im Krieg geschlossen worden war: ${ }^{12}$

(32) Tu-w isamu mëstu sa sïrilu mliku dardu lëtu 1943. Itö lëtu bila wera anu latarïja mažala se wstavït. Po weri, Giovanni Barbarino Śtofänat kupel hïšo tu-ka jë latarïja anu jüdi, tu-w lëtu 1946, majo jïskat no drügi mëstu. Ni ga nalažajo ${ }^{\text {ipf }}$ tuw hï̌̌e Folenkawe-Rinine. Ito prihaja ${ }^{\text {ipf }}$ narëd ta nöwa latarïja, anu jto se nalaža ${ }^{\text {ipf }}$, näs, kaśanjë od te stare latarïje. Śa naredit to nöwo latarïjo, jti ka nisëjo $^{\text {ipf }}$ mliku, majo dät 25 Lire. Tu-w lëtu 1950 se udiwa ${ }^{\text {ipf }}$ ta nöwa latarïja tu-w Bili. Tu-w lëtu 1976, śawöjo potresa, latarija prihaja ${ }^{\text {ipf }}$ śagana, anu na spet se udiwa $^{\text {ipf }}$ tu-w lëtu 1977. Tu-w lëtu 1980, döpö 52 lit, prešident od latarïje, Guido Micelli Rojza, an mara śag̉at latarïjo śawöjo ka jüdi parnašajo ${ }^{\text {ipf }}$ malu mlika. Tu-w lëtu 2001 soči anu eredi od latarïje, šinküwajo ${ }^{\text {ipf }}$ kumünu latarïjo par da an jo porawnej anu an odgej kaśanjë. ${ }^{13}$

'An diesem Ort stellte man bis 1943 Käse aus Milch her. In diesem Jahr war Krieg und die Molkerei musste schließen. Nach dem Krieg kaufte Giovanni Barbarino Štofanat das Haus, wo sich jetzt die Molkerei befindet, und man musste im Jahr 1946 einen anderen Platz suchen. Sie finden ihn in dem Haus von Folenkawa-Rinina. Hier wird die neue Molkerei gebaut und hier findet sich heute die Ausstellung der alten Molkerei. Um die neue Molkerei zu bauen, müssen diejenigen, die Milch bringen, 25 Lire bezahlen. Im Jahr 1950 eröffnet man die neue Molkerei in San Giorgio. Im Jahre 1976 wird die Molkerei wegen des Erdbebens geschlossen und im Jahre 1977 eröffnet man sie wieder. Im Jahre 1980, nach 52 Jahren, muss der Präsident der Molkerei Guido Micelli Rojza die Molkerei schließen, weil die Leute wenig Milch bringen. Im Jahre 2001 schenken die

Andere Fälle finden sich in Baudouin de Courtenay (1895, etwa $§ 372,387$ etc.).

2 Die Passage entstammt der Broschüre „La latteria turnaria di San Giorgio“ (Resia 2016), Comune di Resia.

3 Die Formen prihaja narëd 'wird gebaut' (wörtlich: 'gemacht') und prihaja śagana 'wird geschlossen' stellen Vorgangspassive dar. Nach Meinung aller befragten Informanten ist die hier verwendete Verbform prihaja (zu prihajat 'passieren') inakzeptabel. Es müsste parhaja narëd bzw. parhaja śag்ana (zu parhajat 'kommen') lauten, was einer Lehnübersetzung des italienischen kommen-Passivs entspricht, die allerdings ihrerseits nur relativ selten gebraucht wird. 
Gesellschafter und die Erben der Molkerei die Molkerei der Gemeinde, damit sie sie restauriert und die Ausstellung eröffnet.'

Auf die versuchsweise Ersetzung der ipf. Verben im Text durch entsprechende Perfektiva reagierte eine Informantin positiv, akzeptierte also die Ersetzung, aber nur bei dem Verb se udiwa 'wird eröffnet' und nur nach dem Adverb spet 'erneut'. Wahrscheinlich empfindet man dieses Verb in genau dieser Position als in hohem Grade resultativ und der pf. Aspekt unterstreicht diese Eigenschaft.

\subsection{Gnomische Aussagen}

Abschließend ist festzustellen, dass Resianisch und Slovenisch auch noch im gnomischen Präsens voneinander abweichen. Im Slovenischen erscheint hier der pf. Aspekt relativ häufig, ebenso wie auch bei der Iteration, wenn das Erreichen der inhärenten Grenze der Handlung fokussiert werden soll. ${ }^{14}$

(33) Strog učitelj naredi ${ }^{\mathrm{pf}}$ dobrega učenca.

'Ein strenger Lehrer macht (immer) einen guten Schüler.'

Kdor spi, rib ne ulovi ${ }^{\text {pf }}$.

'Wer schläft, fängt keinen Fisch.'

\section{Bogati umrje ${ }^{\text {pf }}$, reveža pa Bog vzame ${ }^{\text {pf }}$. \\ 'Der Reiche stirbt, den Armen nimmt Gott.'}

Im Resianischen haben wir hingegen eine völlig andere Situation vorliegen: von den analysierten Aphorismen und Sprichwörtern zeigten tatsächlich nur sehr wenige den pf. Aspekt, beispielsweise die folgenden: ${ }^{15}$

\section{Ći Göspud Bug an ti da ${ }^{\text {pf }}$ naga otrokä, an ti daje ${ }^{\text {ipf }}$ pa za ga wredit.}

'Wenn Gott dir ein Kind gibt, gibt er dir auch (die Mittel), es wachsen zu lassen.'

Ko dän wmarjë" ${ }^{p f}$, an spušćüwa ${ }^{\text {ipf }}$ žlïco.

'Wenn jemand stirbt, lässt er den Löffel los.'

An diesen Beispielen ist auch interessant, dass in beiden Fällen auch das Vorhandensein einer so klaren Handlungsfolge das Resianische nicht davon abhält, zwar im ersten Teil den pf. Aspekt zu setzen, im zweiten aber bereits den ipf. Aspekt zu wählen. In Beispiel (36) könnte der Grund darin liegen, dass im ersten Teil auf das Verb ein direktes Objekt im Singular folgt, was üblicherweise die Wahl eines Perfektivums begünstigt, während im zweiten Teil eine iterativ-kontinuierliche, beinahe statische Lesart dominiert. In Beispiel (37) ist einerseits die Wahl des Perfektivums im ersten Teil leicht erklärbar, nämlich als Folge des stark resultativen Charakters des durch wmrit 'sterben' ausgedrückten Sachverhalts. Andererseits ist die Wahl des Imperfektivums im zweiten Teil schwer zu begründen.

14 Diese Beispiele stammen aus dem Internet. Zu weiteren Beispielen vgl. Bojc (1974).

15 Die Beispiele (36)-(37) finden sich in der Rezeptsammlung von Leskovic \& Del Medico (2006: 149, 28), die neben Rezepten auch 98 resianische Aphorismen und Sprichwörter enthält. 


\section{Schluss und Ausblick}

Ungeachtet des vorläufigen Charakters der hier präsentierten Analyse haben sich schon einige interessante Ergebnisse gezeigt. Ganz offensichtlich unterscheidet sich das Resianische in vielfacher Hinsicht vom Slovenischen, der ihm am nächsten stehenden slavischen Standardsprache. Das zeigt sich vor allem darin, dass das Slovenische in allen Kontexten, bei denen im Prinzip beide Aspekte möglich wären, häufig den pf. Aspekt bevorzugt. Dagegen verwenden die Resianer in diesen Kontexten spontan, abgesehen von den bereits erwähnten Spezialfällen (14)-(16), (29), (36)-(37), immer den ipf. Aspekt.

Interessanterweise besteht im Moliseslavischen, einer im Süden Italiens gesprochenen slavischen Minderheitensprache im totalen Sprachkontakt, eine ähnliche Situation. In dieser Mikrosprache ist zwar im Fall der Habitualität, insbesondere bei Handlungsketten, im Präsens der perfektive Aspekt häufig, aber im Fall der expliziten Iteration ist er nicht nachweisbar und beim historischen Präsens stellt er die absolute Ausnahme dar (Breu 2014: 344-347; Breu, Berghaus \& Scholze 2016: 101-102, 104-105). Der Grund hierfür könnte der Kontakt mit dem Italienischen sein, das im Präsens im Gegensatz zu den slavischen Sprachen keine derivative Aspektopposition aufweist, sondern wie das Englische nur zwischen einer nichtprogressiven und einer progressiven Handlung unterscheidet, was hier irrelevant ist.

Die Hypothese eines romanischen Einflusses wird bekräftigt, wenn man die Situation des Resianischen und Moliseslavischen mit der des Burgenlandkroatischen in Österreich, in engem Kontakt mit dem Deutschen, vergleicht. Die Verwendung des pf. Aspekts ist im Burgenlandkroatischen nämlich sowohl bei Iteration wie auch beim historischen Präsens sehr häufig (Breu, Berghaus \& Scholze 2016: 101-107).

Damit folgen das Resianische und das Moliseslavische einerseits und das Burgenlandkroatische andererseits zwei unterschiedlichen Entwicklungslinien, und zwar möglicherweise infolge der unterschiedlichen Struktur der jeweiligen Kontaktsprache. Auf der einen Seite steht das Italienische, in dem es, wie gesagt, keine derivative Opposition gibt und die verbalen Präfixe höchstens wortbildnerische Funktion haben und überhaupt wenig produktiv sind, auf der anderen Seite das Deutsche, das keine aspektuelle Opposition hat, sich aber durch eine ausgeprägte resultativierende Funktion der Präfixe auszeichnet. Wenn wir nun bedenken, dass die Präfixe das typische Bildungsmittel für perfektive Verbformen in den slavischen Sprachen sind, so wird verständlich, dass über sie von der Kontaktsprache her ein gewisser Einfluss auf den bevorzugten Gebrauch der Perfektiva im Präsens ausgehen kann, zumal präfigierte Verbformen im Deutschen problemlos im Präsens gebraucht werden können. Völlig auszuschließen sind aber beim gegenwärtigen Wissensstand von den Kontaktsprachen unabhängige Eigenentwicklungen in den Mikrosprachen nicht, wie sie in Breu, Berghaus \& Scholze (2016: 104) unter Einbeziehung der Besonderheiten der obersorbischen Umgangssprache angenommen werden.

Angesichts dieser Situation wäre es natürlich sehr interessant, zum direkten Vergleich mit dem Resianischen das Kärntnerslovenische heranzuziehen, denn auch hierbei handelt es sich um eine Varietät mit slovenischer Basis, aber eben mit dem Deutschen und nicht dem Italienischen als Kontaktsprache. So könnte der mögliche Einfluss der Dominanzsprachen auf den Gebrauch des perfektiven Aspekts im Präsens insgesamt im direkten Vergleich der Sprachpaarungen Resianisch-Italienisch, Moliseslavisch-Italienisch, Burgenlandkroatisch-Deutsch und Kärtnerslovenisch-Deutsch nachgewiesen werden. 


\section{Literatur}

Apresjan, Ju.D. (1986): Performativy v grammatike i v slovare. Izvestija RAN. Serija literatury i jazyka 45/3. 208-223.

Bajec, A. et al. (1971): Slovenska slovnica. Ljubljana: DZS.

Barentsen, A. (1992): Ob obstojatel'stvach ograničennoj kratnosti dejstvija v russkom jazyke. Cast I. In: Brentsen et al, (Hg.), Studies in Slavic and General Linguistics 17. Amsterdam: Rodopi. 1-66.

Barentsen, A. (1994): Ob obstojatel'stvach ograničennoj kratnosti dejstvija v russkom jazyke. Cast II. In: Brentsen et al. (Hg.), Studies in Slavic and General Linguistics 22. Amsterdam: Rodopi. 1-50.

Benacchio, R. (2002): I dialetti sloveni del Friuli tra periferia e contatto. Udine: Società filologica friulana.

Baudouin de Courtenay, J. (1895): Materialy dlja južnoslovjanskoj dialektologii i ètnografii. I. Rez'janskie teksty. Sanktpeterburg',

Benacchio, R. \& Pila, M. (2015): Glagol'nyj vid v kontekstach neograničennnoj kratnosti v slovenskom jazyke v sopostavlenii s russkim. In: Benacchio, R. (Hg.), Glagol'nyj vid: Grammatičeskoe značenie i kontekst. München: Verlag Otto Sagner. 79-92.

Bondarko, A.V. (1959): Nastojaščee istoričeskoe v slavjanskich jazykach s točki zrenija glagol'nogo vida. In: Vinogradov V.V. (Hg.), Slavjanskoe jazykoznanie. Sbornik statej. Moskva: AN SSSR. 48-58.

Bondarko, A.V. (1971): Vid i vremja russkogo glagola. Moskva: Prosveščenie.

Breu, W. (2000): Zur Position des Slavischen in einer Typologie des Verbalaspekts (Form, Funktion, Ebenenhierarchie und lexikalische Interaktion). In: Breu, W. (Hg.), Probleme der Interaktion von Lexik und Aspekt (ILA). Tübingen. 21-54.

Breu, W. (2011): Language Contact of Minority Languages in Central and Southern Europe: A Comparative Approach. In: B. Kortmann, J. van der Auwera (Hgs.), The Languages and Linguistics of Europe. A Comprehensive Guide. Berlin/New York: De Gruyter. 429-451.

Breu, W. (2014): Funkcii nastojaščego i imperfekta soveršennogo vida i perfekta nesoveršennogo vida $\mathrm{v}$ molizsko-slavjanskom mikrojazyke. Scando-Slavica 60/2. 322-351.

Breu, W., Berghaus, J. \& Scholze, L. (2016): Der Verbalaspekt im totalen Sprachkontakt. Moliseslavisch, Obersorbisch und Burgenlandkroatisch im Vergleich. In: Breu, W \& Reuther, T. (Hg.), Slavistische Linguistik 2014. Referate des XL. Konstanzer Slavistischen Arbeitstreffens in Konstanz/Hegne, 1.-5. September 2014. (= Wiener Slawistischer Almanach, 77). Leipzig, Wien: Biblion Media. 55-116.

Derganc, A. (1992-1993): Neskol'ko zametok v svjazi s performativnymi glagolami v russkom i slovenskom jazykach. Filologija 20-21. 67-74.

Derganc, A. (2002): Nekotorye različija v upotreblenii glagolov soveršennogo - nesoveršennogo vidov v nastojaščem vremeni v russkom i v slovenskom jazykach. In: Kotjurovaja, M.P. \& Ščukina, I.N. (Hg.), Filologičeskie zametki. Sbornik statej. Perm': Permskij Universitet. 52-65.

Derganc, A. (2003): Nekatere razlike v rabi dovršnega oz. nedovršnega vida v ruščini in slovenščini. Slavistična Revija 51. 67-79.

Derganc, A. (2010): Še nekatere razlike v rabi glagolskih vidov v ruščini in slovenščini. Slavistična Revija 58/1. 73-80.

Dickey, S. (2000): Parameters of Slavic Aspect. Standford: CSLI Publications.

Dickey, S. (2003): Verbal Aspect in Slovene. Sprachtypologie und Universalienforschung 56/3. 182-207.

Glovinskaja, M.Ja. (2001): Mnogoznačnost' i sinonimija v vido-vremennoj sisteme russkogo glagola. Moskva: Azbukovnik.

Leskovic, V. \& Del Medico, D. (2006): Rezija. Jëst tu-w rožini dulïni. Cividale del Friuli: Čedad.

Petruchina, E.V. (2009): Russkij glagol: kategorii vida i vremeni. Moskva: MAKS Press.

Pila, M. (2013): La categoria dell'aspetto verbale nel modo indicativo in russo e sloveno: usi e significati a confronto. Tesi di Dottorato. Università degli studi di Padova. Padova.

Plotnikova, O.S. (1975): Glagol'noe vidoobrazovanie $i$ nekotorye osobennosti funkcionirovanija vidov $v$ slovenskom literaturnom jazyke. Dissertacija. MGU. Moskva.

Plotnikova, O.S. (1998): Problemy sopostavitel'nogo izučenija slavjanskogo vida v diachronii. In: Koselev, A.D. \& Čertkova, M.Ju. (Hg.), Tipologija vida: Problemy, poiski, rě̌enija. Moskva: Škola Jazyki Russkoj Kul'tury. 364-370.

Plotnikova, O.S. (1999): Osobennosti funkcionirovanija vidovogo protivopostavlenija v južnoslavjanskich jazykach. In: Usikova, R. P. \& Šešken, A. G. (Hg.), Makedonskij jazyk, literatura i kul'tura v slavjanskom $i$ balkanskom kontekste. Materialy meždunarodnoj rossijsko-makedonskoj naučnoj konferencii (Moskva 15-16 sentjabrja 1998 g.). Moskva: Moskovskij gos. universitet im. Lomonosova. 74-81. 
Wiemer, B. (2014): Upotreblenije soveršennogo vida v performativnom nastojaščem. In: Kazanskij, N.N. (Hg.), Acta linguistica Petropolitana. Trudy instituta lingvističeskich issledovanij. Tipologičeskie issledovanija. Tom X: 3. Sankt Peterburg: Nauka. 90-113.

Steenwijk, H. (1992): The Slovene Dialect of Resia. San Giorgio. Amsterdam, Atlanta, GA: Rodopi.

Steenwijk, H. (1994): Ortografia resiana. Tö jošt rozajanskë pïsanjë. Padova: Cleup.

Stunová, A. (1993): A Contrastive Analysis of Russian and Czech Aspect: Invariance vs. Discourse. Amsterdam: Academisch proefschrift.

Zaliznjak, A.A. \& Šmelev, A.D. (2000): Vvedenie v russkuju aspektologiju. Moskva: Jazyki Russkoj Kul'tury.

\section{Quellen}

Bojc, E. (1974): Pregovori in reki na Slovenskem. Ljubljana: Državna Založba Slovenije. Buccati, D. (2010): Tatarskaja pustynja. Sankt-Peterburg: Astrel'.

Buzzati, D. (2004): Tatarska puščava. Ljubljana: Delo.

Dovlatov, S. ([1986] 2010): Čemodan. Sankt-Peterburg: Azbuka klassika.

Dovlatov, S. (2006): Tujka. Kovček. Maribor: Litera.

Kosmač, C. ([1953] 2001): Pomladni dan. Ljubljana: DZS.

Kosmač, C. (1988) : Vesennij den'. In: Vagapova, N. (Hg.), C. Kosmač. Izbrannoe. Moskva: Raduga. 19-169.

Zimin, V.I. (2008): Slovar'. Tezaurus russkich poslovic, pogovorok i metkich vyraženij. Moskva: AST-PRESS. 



\title{
Das sogenannte jako recitativum und seine paradigmatischen Verhältnisse zu übrigen Mitteln der altkirchenslavischen Syntax*
}

\author{
Pino Marco Pizzo (Mainz)
}

\begin{abstract}
Das sogenannte jako recitativum ist Gegenstand diverser Untersuchungen im Rahmen der altkirchenslavischen, russisch-kirchenslavischen und altrussischen Syntax. Das besondere Interesse an diesem sprachlichen Phänomen ist bisher vor allem durch die syntaktische Distribution des jako begründet, da es sowohl als (unterordnender) Komplementierer als auch zur Einleitung syntaktisch beigeordneter Redewiedergaben auftritt, und somit in letzterem Kontext nicht als prototypischer Komplementierer kategorisiert werden kann. Mehrere bestehende Beschreibungen dieses Phänomens zielen darauf ab, das jako recitativum und seine ausbleibende Realisierung im Rahmen der Redewiedergabe auf ihre Funktionen hin zu untersuchen: Das Ergebnis schließt je nach altslavischer Varietät diverse Funktionsbereiche ein, modale Funktionen (i.w.S.) und die pragmatische Beschreibungsebene werden jedoch in allen Fällen herangezogen, sodass sich hierin gewisse Kernfunktionen dieses Phänomens vermuten lassen müssen. Der folgende Beitrag möchte den bestehenden Forschungsdiskurs bezüglich dieses sprachlichen Phänomens um eine breitere systeminterne Analyse ergänzen. Hierbei werden für den altkirchenslavischen Codex Zographensis die jeweiligen Folgesätze zu glagolati 'sprechen, reden', dem dort frequentesten verbum dicendi, auf ihre syntaktischen Mittel hin untersucht und in ihren Funktionsweisen analysiert. Das Ergebnis ist eine Verteilung diverser Mittel der altkirchenslavischen Syntax, die ein paradigmatisches Verhältnis eingehen, um Oppositionen innerhalb der illokutiven Modalität und das Merkmal der Wahrheitsbedingungen zu realisieren. Schließlich wird ein Ausblick auf diverse mögliche Untersuchungsrichtungen zu diesem sicherlich weiterhin bearbeitungswürdigen Gegenstandsbereich gegeben.
\end{abstract}

\section{Einführung}

Das sogenannte jako recitativum findet sich in der umfangreichen (insg. fünfbändigen) Arbeit Večerkas (2002: 421) zur altkirchenslavischen Syntax im Kontext einer dreigliedrigen Systematisierung der oratio semirecta zugeordnet, wobei diese den „Übergangstyp zwischen der oratio recta und der oratio obliqua [darstelle]“. Zur Einbettung wiedergegebener Rede in den aktuellen Diskurs werde bei der oratio semirecta ,zum einen als Merkmal ihrer syntaktischen A b hängigkeit von der neuen Textumgebung das entsprechende Binde mitte ${ }^{1}$ verwendet, zum anderen begegn[e] man aber als das Merkmal ihrer syntaktischen Un abhängigkeit in derartigen syntaktischen Formationen der Nichtverschiebung der grammatischen

Für die kritische Lektüre des Manuskripts danke ich Patrick Mächler.

An dieser Stelle wird mit dem „Bindemittel“ die Realisierung der Partikel jako bezeichnet. Im Folgenden wird dieser Terminus beibehalten, da er vorerst davor bewahrt, Aussagen über den syntaktischen Status der jeweiligen Realisierungen treffen zu müssen. 
Person und des grammatischen Modus (d.h. des synthetischen Imperativs in die $d a$-Wendung)““. Bei Večerka (ebd.) findet sich hierzu - unter vielen anderen - folgendes Beispiel: ${ }^{2}$

$$
\begin{array}{lllll}
\text { onb že } & \text { 'glaše } & \text { ěko azb esmb } \\
\text { er } & \text { aber } & \text { sagen:3SG.IMPF ěko ich } & \text { sein:1SG.PRS } \\
\text { 'er selbst aber sagte: »Ja, ich bin's. «' (Jh. 9,9) }
\end{array}
$$

In dieser Konstruktion ist offensichtlich, dass die origo des Satzes vor dem jako von der origo der Redewiedergabe (nach dem jako) abweicht, da die entsprechende Personaldeixis ${ }^{3}$ nicht auf eine gemeinsame origo bezogen werden kann. Klassisch wird dieses Verhältnis der divergierenden origines als direkte Rede oder eben oratio recta bezeichnet. Allerdings scheint es auffällig, dass an dieser Stelle ein Element auftritt, welches aus der oratio obliqua bekannt ist.

In der Tat besitzt die altkirchenslavische Sprache das Bindemittel jako ebenfalls als Mittel des Ausdruckes der oratio obliqua, welche nach Večerkas (2002: 416) Definition dadurch gekennzeichnet ist, dass „die in einer anderen Situation verwirklichte Aussage in der neuen Situation auf die Weise wiedergegeben [wird], daß sie auch syntaktisch zum integralen Bestandteil der neuen Sprachschicht wird." Hierbei beschränken sich die Bindemittel des Altkirchenslavischen für die oratio obliqua keineswegs alleinig auf das in diesen Fällen als subordinierend zu bezeichnende jako, vielmehr eröffnet sich dem Altkirchenslavischen in dieser Funktion eine Vielzahl sprachlicher Mittel. Wiederum gibt Večerka (2002: 420) ein entsprechendes Beispiel: ${ }^{4}$

$$
\begin{array}{llllll}
i \quad \text { zaprěti } & \text { imb } & d a & \text { ničesože } & n e \\
\text { und gebieten[3SG.AOR] } & \text { ihnen[DAT.PL] } & d a & \text { nichts:GEN.SG } & \text { NEG } \\
\text { vbzemljotb } & \text { na } & \text { pqtb } & & \\
\text { nehmen:3PL.PRS } & \text { auf } & \text { Weg:AKK.SG } & \\
\text { 'und [er] gebot ihnen, nichts auf den Weg mitzunehmen' (Mk. 6,8) }
\end{array}
$$

Das Auftreten der verschiedenen Bindemittel ist hierbei jedoch nicht willkürlich: ${ }^{5}$ Večerka (2002: 399) benennt als ,häufigstes Klassifizierungskriterium für die abhängigen Inhaltssätze [...] ihre illokutive Modalität“, wobei er weiter „,deklarative, interrogative oder Imperativund Optativsätze“ unterscheidet. Die entsprechenden Bindemittel sind hierbei „die Konjunktion jako, vereinzelt auch [j]eže für die deklarativen Inhaltssätze, die Fragepartikel li und

2 Die altkirchenslavischen Belege sind dem digitalen Corpus TOROT (https://nestor.uit.no/users/sign_in) entnommen (letzter Zugriff August 2016) und teilweise in gekürzter Form wiedergegeben; für den weiteren Kontext helfen die Bibelstellenangaben. (Alle altkirchenslavischen Belegstellen der vorliegenden Arbeit entstammen dem Codex Zographensis.) Die neuhochdeutsche Übersetzung ist hingegen der digitalen Version Menges (http://www.bibelwissenschaft.de/de/online-bibeln/menge-bibel/bibeltext; letzter Zugriff ebenfalls August 2016) entnommen und ggf. dem altkirchenslavischen Beleg entsprechend gekürzt wiedergegeben. Abänderungen (in wenigen Fällen) erfolgen innerhalb eckiger Klammern. - Schließlich entspricht die Glossierung dem „Leipziger Standard“. Allerdings mit Ergänzungen, bspw. für Aorist (AOR) und Imperfekt (IMPF), einer Orientierung an der lateinischen Terminologie, bspw. Präteritum (PRT) statt past (PST), und unter Auslassung für hiesige Zwecke irrelevanter Angaben, bspw. Genusangaben.

3 Die Personaldeixis drückt sich selbstverständlich nicht nur durch die realisierten Personalpronomina aus, sondern betrifft alle Markierungen der grammatischen Person (also auch Possessivpronomina und die verbale Morphologie).

4 Es sei angemerkt, dass Večerka (2002: 416-420) die Beispiele einer syntaktisch nicht ambigen oratio obliqua mit dem Bindemittel jako konstruiert. Einzige Ausnahme bildet éin Beleg aus der Apostelgeschichte (s. ebd.).

5 Večerka (2002: 399) bemerkt allerdings, dass die im Folgenden aufgeführte Distribution der sprachlichen Mittel nicht immer strikt eingehalten sei, sondern Abweichungen (aufgrund funktionaler „Berührungsflächen“) aufzeigen könne. (Belege bleiben an dieser Stelle jedoch aus.) 
die Konjunktionen ašte und [j]eda sowie die $k$-haltigen Fragepronomina und -adverbien für die interrogativen Inhaltssätze und die Konjunktion $d a$ für die imperativischen/optativischen Inhaltssätze“".

Interessanterweise ordnet Večerka (2002: 422f.) aus den soeben aufgeführten Bindemitteln kako und $d a$ ebenfalls als (wenn doch seltenere) mögliche recitativa ein. Zudem lassen sich auch einige der vermeintlichen Bindemittel, wie bspw. $l i$ und $d a$ oder die Fragepronomina, in nicht komplexen (bzw. in parataktischen) Konstruktionen vorfinden. Die syntaktische Kategorisierung trägt folglich keine entscheidende Bedeutung für die Distribution dieser Bindemittel. Vielmehr scheint tatsächlich die illokutive Modalität die Distribution der sprachlichen Mittel zu bestimmen. Ähnlich spricht Daiber (2013: 115) für das jako im Altkirchenslavischen des Codex Marianus von einer ,modalen Funktion, nämlich die Anzeige der Übernahme der Illokution“. Des Weiteren bringt für das Russisch-Kirchenslavische Daiber (2009: 369) die Verwendung des jako recitativum mit den „Wahrheitsbedingungen des wiedergegebenen Redebeitrages" in einen Zusammenhang, was sich für das Altrussische ebenso in Perelmutter (2009: 119) wiederfindet. Letztere (2009: 114ff.) ordnet im Rahmen jener Arbeit dem Auftreten des jako recitativum allerdings noch weitere Funktionen zu, die dem Bereich der Modalität (i.w.S.) ${ }^{6}$ zugeordnet werden können (oder diesem zumindest nahe stehen): Offensichtlich erstrecken sich die Funktionen der oben genannten Bindemittel weit über den syntaktisch orientierten dreigliedrigen Kategorisierungsrahmen aus oratio recta, semirecta und obliqua, wobei sie gleichzeitig Funktionen aus den Bereichen der Modalität und der Pragmatik berühren (oder gar übernehmen).

Entsprechend der Komplexität, die aus den verflochtenen syntaktischen, modalen und pragmatischen Beschreibungsebenen resultiert, erfreut sich der Forschungsdiskurs um das sogenannte jako recitativum durchaus auch in rezenter Zeit einer regen Bearbeitung, wobei vor allem den Funktionen seiner Realisierung im Rahmen der oratio semirecta und dessen Ausbleiben in demselben Kontext besondere Aufmerksamkeit geschenkt wird. Die paradigmatische Stellung zu den übrigen, oben bereits erwähnten, Bindemitteln bleibt hingegen oftmals unbeachtet. ${ }^{7}$ In der folgenden Untersuchung soll diesem Gegenstand nachgekommen werden, indem am Beispiel des Codex Zographensis eine Darstellung der Distribution derjenigen altkirchenslavischen Mittel erfolgt, die sich in einem paradigmatischen Verhältnis mit dem Bindemittel jako befinden. Hierfür wird der Kontext untersucht, der unmittelbar in Anschluss an das im altkirchenslavischen Codex Zographensis frequenteste verbum dicendi auftritt, nämlich glagolati 'sprechen, reden'.

\section{Fallstudie}

Der Codex Zographensis gehört zu den ältesten Textzeugen des Altkirchenslavischen (älteste Schicht Ende 10./Anfang 11. Jh.) und entspringt vermutlich dem westlichen Gebiete des Ersten Bulgarischen Reiches. Es ist ein eklektischer Codex, insofern er zwei spätere Ergänzungen aufweist, die ältere in glagolitischer, die jüngere in kyrillischer Schrift (vgl. Schaeken \& Birnbaum 1999: 95). Das verbum dicendi, welches für die Untersuchung den konstanten Kontext darstellt, findet sich hierin in 959 Vorkommnissen zum Lemma glagolati

6 Die jeweilige Terminologie der verschiedenen Arbeiten bezüglich der Modalität (u.Ä.) kann an dieser Stelle nicht diskutiert werden. Mit dem Begriff der Modalität seien aber an dieser Stelle auch die Bereiche der epistemischen Modalität und der Evidentialität einbegriffen.

7 Eine Ausnahme hierzu bildet Daiber (2008), der die komplementäre Distribution von jako und eže im Russisch-Kirchenslavischen untersucht. 
wieder und stellt damit - nach dem Lemma byti 'sein' mit 1902 Vorkommnissen - das frequenteste Verbum im Codex Zographensis dar, womit es ebenfalls das frequenteste verbum dicendi ist. ${ }^{8}$

Für den ersten Teil der Betrachtung (s. 2.1-2.4) sind alle Kontexte aus dem Evangelium nach Matthäus auf ihre formalen Mittel und deren funktionale Verteilung untersucht worden. In einem zweiten Schritt (s. 2.5) wurde dieses Material um die Belegstellen aus den übrigen drei Evangelia ergänzt, die für hiesige Zwecke relevantes Sprachmaterial enthalten. Bei der Analyse der somit erhaltenen Belege sind grundsätzlich zwei Eigenschaften festgehalten: Erstens sind die syntaktischen Verhältnisse über den Abgleich der origo des Satzes mit dem Verbum glagolati (= potentieller Matrixsatz) und der origo des darauf folgenden (Inhalts-) Satzes festgehalten. ${ }^{9}$ Zweitens ist entsprechend der zuvor beschriebenen Paradigmatik die konkrete Realisierung der altkirchenslavischen Mittel dargestellt, wobei nicht nur Bindemittel, sondern auch asyndetische (Inhalts-)Sätze unter besonderer Berücksichtigung der morphologischen Eigenschaften des Verbums aufgeführt sind. ${ }^{10}$ Die daraus resultierende Aufteilung soll nun anhand des tatsächlichen Materials dargestellt werden.

\subsection{Belege ohne Bindemittel und mit finiter Verbmorphologie}

Für diesen ersten Typus liegen zahlreiche Belege vor, die dadurch gekennzeichnet sind, dass keine Bindemittel auftauchen und das Verbum der folgenden Redewiedergabe mit (indikativer und imperativer) finiter Verbmorphologie ausgedrückt wird. Hypotaktische Verhältnisse lassen sich nicht bezeugen, bestenfalls sind die Belege syntaktisch ambig. Oftmals genügt die Personaldeixis aber auch zur Feststellung des parataktischen Verhältnisses:

$$
\begin{aligned}
& \text { vbzbraněše emu } \quad \text { glę } a z b \\
& \text { wehren:3SG.IMPF ihm[DAT.SG] sagen:PTCP.PRS.ACT.NOM.SG ich } \\
& \text { trěbujo otb tebe krbstiti sę } \\
& \text { benötigen:1SG.PRS von } \operatorname{dir}[\text { GEN.SG] taufen:INF REFL } \\
& \text { 'der wollte ihm aber nicht zu Willen sein und sagte: »Ich müßte von dir getauft } \\
& \text { werden «' (Mt. 3,14) }
\end{aligned}
$$

$\mathrm{Zu}$ diesem Typus sind auch diejenigen Belege zu stellen, in denen die dem Verbum glagolati folgende Rede mit einer Interjektion (4) oder einem Vokativ (5) beginnt; hierbei müssen die syntaktischen Verhältnisse bereits vor Analyse der angewandten Personaldeixis als unabhängig gelten, da Interjektionen und Vokative per definitionem aus den sie umgebenden syntakti-

8 Wie bereits bzgl. der altkirchenslavischen Belegstellen festgehalten, sind auch die Informationen über die Frequenz der einzelnen Verba dem Corpus TOROT entnommen (vgl. Fußnote 2). Für eine entsprechende Frequenzliste sei an dieser Stelle Hanne Martine Eckhoff gedankt.

9 Hierbei ist eingangs von drei möglichen Ergebnissen ausgegangen worden: Die nachweisliche Divergenz der origines, der nachweisliche Zusammenfall der origines und letztlich die ambigen Fälle, die nicht zwischen dem ersten und dem zweiten Falle entscheiden lassen.

10 Neben einer Vielzahl an Bindemitteln verfügt das Altkirchenslavische zudem über mehrere finite und infinite Formen des Verbums, wobei sich für die weitere Betrachtung die finiten Indikativformen, die Infinitive und die Partizipialformen als relevant herausgestellt haben. - Zur Datenauswertung ist im Allgemeinen anzumerken, dass aufgrund der im Verhältnis zum gesamten Codex nur „stichprobenartigen“ Analyse auf die konkrete Angabe quantitativer Verhältnisse i.d.R. verzichtet wird; Adverbia der Art „oftmals“, „selten“ (etc.) sollen dem Leser jedoch eine erste Einschätzung ermöglichen. 
schen Verhältnissen losgelöst sind. ${ }^{11}$ Eine durch Zusammenfall der origines belegte hypotaktische Unterordnung ist dementsprechend nicht belegt.

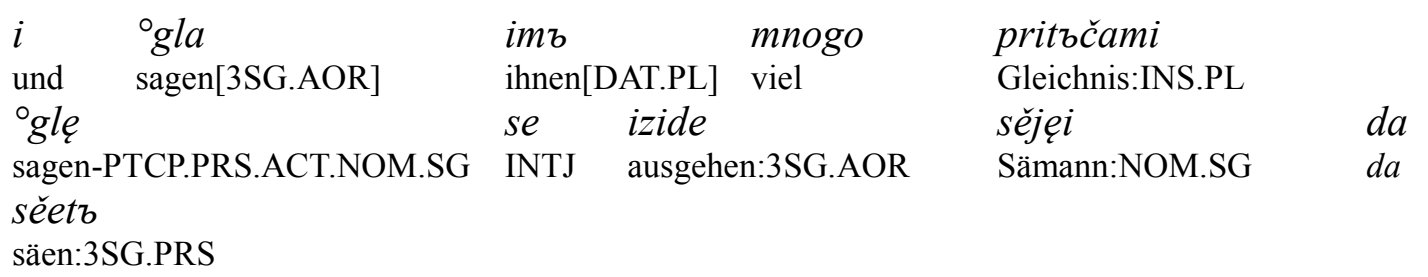

'da redete er mancherlei zu ihnen in Gleichnissen mit den Worten: »Seht, der Sämann ging aus, um zu säen«’ (Mt. 13,3)

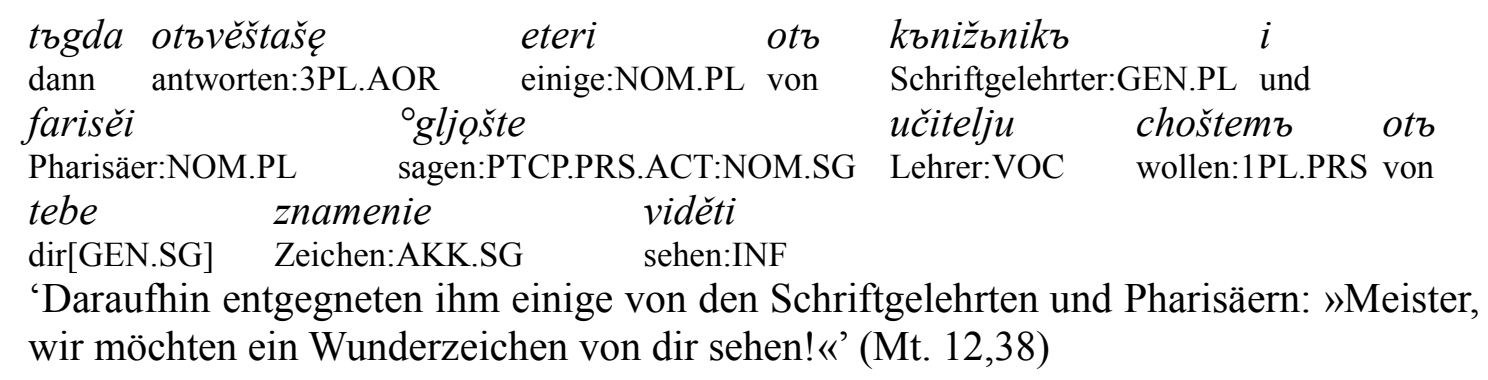

Schließlich ist diesem Typus auch diejenige Rede zuzuordnen, die mit einem finiten imperativen Verbum Ausdruck findet. Hierbei wird der Imperativ traditionell als hinreichendes Merkmal aufgeführt, um den nicht unterordnenden Status als nachgewiesen zu erklären (vgl. zu Beginn dieses Beitrags Večerka 2002: 421). Diese Annahme ist jedoch problematisch, wenn sie nicht am Sprachmaterial selbst nachgewiesen wird, da der slavische Imperativ formal die Kontinuante des indogermanischen Optativs (Grammem) darstellt und somit zumindest bis zu einem gewissen Zeitpunkt durchaus einen Modus untergeordneter syntaktischer Strukturen dargestellt haben muss (vgl. Vondrák 1928: 119ff., 394ff.). Nun ist es aber eine grundsätzliche methodologische Frage, inwiefern man überhaupt die Nichterfüllung einer (hier syntaktischen) Eigenschaft, welche in diesem Falle dem Auftreten des slavischen Imperativgrammems in hypotaktischen Konstruktionen entspricht, nachweisen kann. In hiesigem Textzeugen lassen sich nämlich für den untersuchten Kontext durchaus syntaktisch ambige Fälle vorfinden:

$$
\begin{aligned}
& \text { Azъ že }{ }^{\circ} \text { gljo vamı ljubite vragy vašę } \\
& \text { ich aber sagen:1SG.PRS euch[DAT.PL] lieben:2PL.IMP Feinde:AKK.PL eure:AKK.PL } \\
& \text { 'Ich dagegen sage euch: Liebet eure Feinde' (Mt. 5,44) }
\end{aligned}
$$

A priori dieses Grammem als Mittel der Unterordnung auszuschließen, deutet nicht nur auf eine Vernachlässigung der historisch-vergleichenden Erkenntnisse, sondern übergeht vor allem ein entscheidendes methodisches Problem: Allzu oft ist das syntaktische Verhältnis der in diesem Beitrag untersuchten Konstruktionen ambig. ${ }^{12}$ Nun ist bereits eine Beweisführung ex

11 Tatsächlich werfen Interjektionen und Vokative hinsichtlich ihrer syntaktischen Kategorisierung diverse Fragen auf. Für einen ersten Einblick bezüglich des syntaktischen Verhaltens s. Graf (2011: 115ff.) zu den Interjektionen, sowie Sonnenhauser \& Noel (2013: 9-13) zum Vokativ.

12 Tatsächlich weist auch Večerka (2002: 420) zumindest in Zusammenhang mit dem Bindemittel jako darauf hin, dass im Falle der ambigen Personaldeixis eine „eigentlich zweideutige“ syntaktische Konstruktion vorliegt. Seine an dieser Stelle relativierende Sprache überrascht, da keinerlei Kriterien zur Disambiguierung angeführt werden. Diese Tatsache hat auch in anderen Arbeiten bestand, wie beispielsweise Daiber (2013: 111), wo die vermeintlichen Objektsätze in keinem der angegebenen Fälle tatsächlich eine nachweisliche hypotak- 
negativo methodologisch diskutabel und wird nicht immer als stichhaltig empfunden, wieviel mehr sollte also auch das Auftreten zahlreicher ambiger syntaktischer Verhältnisse mehr Gewichtung finden.

\subsection{Belege mit Bindemittel und finiter Verbmorphologie: jako, jeda, ašte und da}

In diesem Abschnitt sollen vier altkirchenslavische Bindemittel (nämlich jako, jeda, ašte und $d a)$, die in dem hier gewählten Kontext nach dem frequentesten verbum dicendi auftreten, in ihrem jeweiligen Distributionsverhalten dargestellt werden. Allgemein gilt, dass nach dem jewieligen Bindemittel sowohl bei nachweislich divergierenden origines als auch bei syntaktisch ambigen Konstruktionen eine finite indikative Verbform folgt. Nachweislich hypotaktische Konstruktionen treten parallel zu den Belegen des Typus in 2.1. wiederum nicht auf. ${ }^{13}$ Zudem scheint bestätigt, dass eine Distribution der Bindemittel nach ihrer illokutiven Modalität erfolgt. Für deklarative Sätze tritt jako als einziges Bindemittel auf: ${ }^{14}$

$$
\begin{aligned}
& \begin{array}{llllllll}
{ }^{\circ} g l j g & \check{z} e & \text { vamb } & \check{e} k o & \text { mnodzi } & \text { otb } & \text { vbstokb } & i \\
\text { sagen:1SG.PRS } & \text { aber } & \text { euch[DAT.PL] } & e \check{k o} & \text { viele:NOM.PL } & \text { von } & \text { Osten:GEN.PL } & \text { und }
\end{array} \\
& \text { zapads pridotb } \\
& \text { Westen:GEN.PL kommen:3SG.PL } \\
& \text { ‘ich sage euch aber: Viele werden von Osten und Westen kommen’ (Mt. 8,11) }
\end{aligned}
$$

Dieser Beleg ist den obigen Anmerkungen $\mathrm{zu}$ den Gesamtverhältnissen entsprechend syntaktisch ambig und typisch für das erste Evangelium des Codex Zographensis, wo sich nämlich für den hiesigen Kontext im Gegensatz zum Codex Marianus keine nachweislichen recitativa vorfinden. Zumindest stellenweise scheint der Zographensis gegenüber anderen Überlieferungen eindeutige recitativa zu vermeiden (vgl. Večerka 2002: 421).

Gegenüber den deklarativen Sätzen weisen die interrogativen Sätze zwei Bindemittel auf, auch wenn diese insgesamt wesentlich seltener sind. Diese niedrige Vorkommnisfrequenz steht sicherlich in Verbindung mit der Semantik des hier gewählten Verbums. ${ }^{15}$ Die Partikel ašte wird im Altkirchenslavischen vor allem als Konjunktion zur Einleitung von Konditionalsätzen verwandt und tritt in dieser Position regelmäßig auf; doch auch ihre Verwendung als Bindemittel nach dem Verbum glagolati liegt vor:

$$
\begin{aligned}
& i \text { se }{ }^{\circ} \mathrm{c} k b \text { be } \quad \text { tu } \\
& \text { und INTJ Mensch:NOM.SG sein.3SG.IMPF DEM[AKK.SG] Arm:AKK.SG } \\
& \text { imy sucho } i \text { vъprosiše } i \\
& \text { haben:PTCP.PRS.ACT.NOM.SG trocken:AKK.SG und fragen:3PL.AOR ihn[AKK.SG] }
\end{aligned}
$$

tische Konstruktion darstellen, sondern ebenso als Belege der oratio semirecta interpretiert werden können. Des Weiteren ist hiermit in einem zweiten Schritt die theoretische Annahme komplementloser hypotaktischer Konstruktionen verbunden, die an dieser Stelle als gültig vorausgesetzt wird [vgl. (6)].

13 Dieser Befund ist hauptsächlich für das Bindemittel jako relevant, da die übrigen Bindemittel allzu selten auftreten, um überhaupt ex negativo einen Eindruck erhalten zu können (vgl. aber auch die vorangehende Fußnote 12).

14 Im Evangelium nach Matthäus tritt jako im hier untersuchten Kontext einmalig als Vergleichspartikel auf und wird mit dem Klitikon že erweitert (s. Mt. 6,7).

15 Vgl. hierzu auch Daiber (2008: 29): „Die illokutive Modalität des Inhaltssatzes hängt gewöhnlich an der Semantik des Verbs im Einleitungssatz.“ 


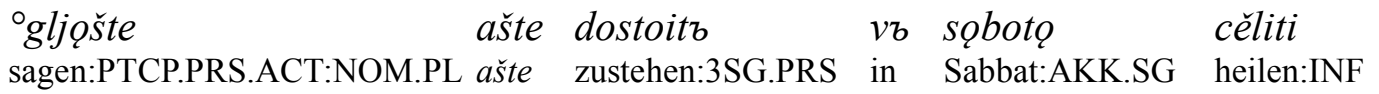

'Da war ein Mann, der einen gelähmten Arm hatte; und sie richteten die Frage an ihn: »Darf man am Sabbat heilen? «' (Mt. 12,10)

Da an dieser Stelle die syntaktische Zuordnung ambig bleibt, kann auch die Übersetzung als hypotaktische Konstruktion erfolgen: „Und sie richteten die Frage an ihn, ob man am Sabbat heilen dürfe.“ Ähnlich scheint auch das zu den interrogativen Inhaltssätzen gehörende Bindemittel jeda belegt zu sein:

$\begin{array}{lllll}i \quad \text { divlěacho } & s e & v s i & n a r o d i & \\ \text { und wundern:3PL.IMPF } & \text { REFL } & \text { alle:NOM.PL } & \text { Volk:NOM.PL } & \\ \text { ogljgšte } & e d a & c b & \text { estb } & { }^{\circ} \text { snb } \\ \text { sagen:PTCP.PRS.ACT:NOM.PL } & \text { jeda } & \text { DEM[NOM.SG] sein:3SG.PRS } & \text { Sohn:NOM.SG } \\ & \end{array}$

${ }^{\circ}$ davb

David.ADJ:NOM.SG

'Da geriet die ganze Volksmenge vor Staunen außer sich und sagte: »Sollte dieser nicht doch der Sohn Davids sein? «' (Mt. 12,23)

Bei dieser Partikel scheint es auffällig, dass der Inhaltssatz der Redewiedergabe mit einem gewissen „Zweifel“" versehen ist, wie in (9) durch das Lexem diviti se 'sich wundern' suggeriert wird. Es finden sich weitere Belege dieser Art: ${ }^{16}$

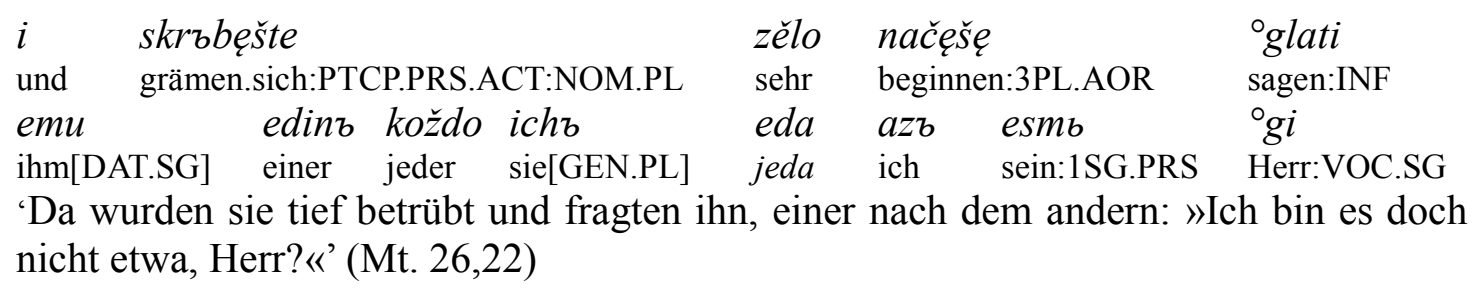

Schließlich taucht im Zusammenhang mit dem Verbum glagolati in dem hier besprochenen Textabschnitt das Bindemittel $d a$ auf, welches entsprechend den vorangegangenen Annahmen Imperativ- und Optativsätzen zuzuordnen ist:

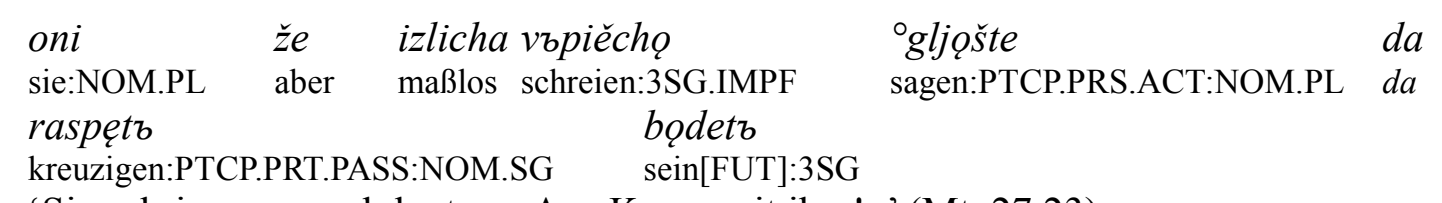

'Sie schrien nur noch lauter: »Ans Kreuz mit ihm!«' (Mt. 27,23)

Die syntaktische Konstruktion ist auch in diesen Fällen ambig und könnte gleichfalls hypotaktisch interpretiert werden. In Zusammenhang mit dem Verbum glagolati ist die Beleglage in dieser Hinsicht aber äußerst spärlich. Sicherlich ist die Verbsemantik wieder entscheidend, wie es auch für die interrogativen Inhaltssätze festzustellen ist. Eine Untersuchung derselben Art mit dem Verbum rešti 'sagen' kann diesbezüglich gewiss ertragreich sein, da hierfür ein erster Blick in das Korpus, gegenüber den Verhältnissen der Folgesätze zu glagolati, für das

16 Wie zu bemerken ist, tritt jeda sowohl in syntaktisch ambigen (9) als auch in syntaktisch eindeutig nicht untergeordneten Inhaltssätzen (10) auf, was an dieser Stelle wieder an den divergierenden origines ersichtlich ist. 
Bindemittel $d a$ eine deutliche Präferenz erkennen lässt. Eine divergierende (lexikalische und/ oder grammatische) Semantik der beiden Verba scheint vorzuliegen. ${ }^{17}$

\subsection{Belege ohne Bindemittel und mit infiniter Verbmorphologie}

Inhaltssätze mit ausschließlich infiniter Verbmorphologie in Folge eines glagolati sind nur marginal vertreten. Trotzdem ist durch die Belege ersichtlich, dass eine gewisse formale Varianz möglich ist und diese vereinzelten Belege zudem diversen illokutiven Modalitäten zugeordnet werden müssen. Hierbei bestehen für die deklarativen Inhaltssätze zwei formale Realisierungen, die aufgrund der Ähnlichkeit der Belege als miteinander konkurrierende Realisierungen kategorisiert werden können:

\begin{tabular}{lllll} 
kogo & ${ }^{\circ g l j g t b}$ & $m e$ & ${ }^{\circ} \check{c} c i$ & sošta \\
wen & sagen:3SG.PRS & mich & Leute:NOM.PL & sein:PTCP.PRS.ACT:AKK.SG \\
${ }^{\circ}$ sna & \multicolumn{0}{c}{${ }^{\circ}$ skago } \\
Sohn:AKK.SG & menschlich:AKK.SG
\end{tabular}

'Für wen halten die Leute den Menschensohn?' (Mt. 16,13)

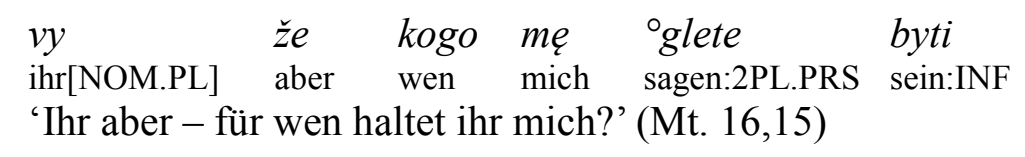

Im ersten Falle (12) liegt ein Accusativus cum Participio (AcP) vor, im zweiten Falle (13) ein Accusativus cum Infinitivo (AcI). In beiden Belegen scheint es ausschlaggebend, dass im Gegensatz zu den bisherigen deklarativen Inhaltssätzen, die als Bindemittel jako fordern, der Sprecher mit gewisser Skepsis die Beantwortung seiner Frage erwartet und somit offensichtlich an deren Richtigkeit zweifelt.

Des Weiteren seien als übrige infinite Konstruktionen der Dativus cum Infinitivo (DcI) und der bloße Infinitiv aufgeführt, die in den Belegen ausschließlich negiert auftreten. Die illokutive Modalität dieser infiniten Inhaltssätze entspricht den Imperativ- und Optativsätzen:

\begin{tabular}{|c|c|c|c|c|c|c|c|}
\hline $\begin{array}{l}a z b \\
\text { ich }\end{array}$ & $\begin{array}{l}\text { že } \\
\text { aber }\end{array}$ & $\begin{array}{l}{ }^{\circ} \text { gljo } \\
\text { sagen:1SG.PRS }\end{array}$ & $\begin{array}{l}\text { vamb } \\
\text { euch[DAT.PL] }\end{array}$ & $\begin{array}{l}n e \\
\text { NEG }\end{array}$ & $\begin{array}{l}\text { klęti } \\
\text { schwören:INF }\end{array}$ & $\begin{array}{l}s e \\
\text { REFL }\end{array}$ & $\begin{array}{l}\text { vamb } \\
\text { euch[DAT.PL] }\end{array}$ \\
\hline
\end{tabular}
$a z b \quad \check{z} e$
že $\quad{ }^{\circ} g l j Q$
vamb
ne
protiviti
se zblu
'ich dagegen sage euch: Ihr sollt dem Bösen keinen Widerstand leisten' (Mt. 5,39)

In (15) ist das logische Subjekt des Folgesatzes nicht realisiert, sodass für diesen Beleg ebenfalls ein DcI (aber eben ohne Realisierung des logischen Subjekts im Dativ) angenommen wird: die beiden Bildungstypen können (zumindest zu hiesigen Zwecken) folglich zusammengefasst werden.

17 An dieser Stelle ist vorerst nur von den Verhältnissen im Codex Zographensis die Rede. 


\subsection{Zusammenfassung der Ergebnisse im Evangelium nach Matthäus des Codex Zographensis}

Die vorliegenden Belege in unmittelbarer Folge nach dem verbum dicendi glagolati zeigen auf syntaktischer Ebene in erster Linie eine Dichotomie zwischen komplexen Satzkonstruktionen mit divergierenden origines und solche mit syntaktisch ambigen Konstruktionen. Wichtig ist nochmals festzuhalten, dass Belege mit einer (durch eindeutigen Zusammenfall der origines) nachgewiesenen hypotaktischen Struktur in diesem Kontext nicht bestehen. Des Weiteren zeigt sich in Bezug auf die Bindemittel und die Verbmorphologie des Folgesatzes eine Vielfalt an Realisierungen, die durch verschiedene Faktoren motiviert ist. Wichtig ist hierbei die illokutive Modalität, welche sich in ein dreigliedriges System zwischen deklarativen, interrogativen und Imperativ- und Optativsätzen aufteilen lässt. Während für die erste und die letzte illokutive Modalität jeweils éine Partikel verzeichnet ist, besteht im Bereich der interrogativen Inhaltssätze eine formale Opposition zwischen zwei Bindemitteln, ašte und jeda. Diese lässt sich allerdings auflösen, wenn man bedenkt, dass das Bindemittel jeda einen gewissen Zweifel des Sprechers zum Ausdruck bringen möchte. Eine tabellarische Darstellung sieht vorerst wie folgt aus:

\begin{tabular}{|c|c|c|}
\hline deklarativ & interrogativ & direktiv \\
\hline jako & $a s ̌ t e, j e d a[+Z w e i f e l]$ & $d a$ \\
\hline
\end{tabular}

Tab. 1

Die Darstellung in Tab. 1 vereint nun alle Bindemittel, die besprochen worden sind. Zusätzlich beinhaltet der Codex Zographensis jedoch im ersten Evangelium Folgesätze, die zwar kein Bindewort aufweisen, aber dafür durch eine infinite Verbmorphologie markiert sind (vgl. 2.3.). Für die deklarativen Sätze scheint der AcP und der damit offenbar konkurrierende AcI einen Ausdruck des Zweifels seitens des Sprechers zu suggerieren, wie wir es auch für die interrogativen Inhaltssätze als Merkmal vorfinden. Somit kann neben der illokutiven Modalität das Merkmal [ \pm Zweifel] hinzugefügt werden, wenn jako und ašte tatsächlich das Merkmal [+Zweifel] nicht aufweisen. Für jako gilt diese Bedingung als Ausdruck des deklarativen Inhaltssatzes sicherlich, da ein Zweifel des Sprechers gegenüber der Aussage nicht beobachtet werden kann. Wie verhält es sich aber mit ašte? Hierzu soll die Belegstelle aus (8) nochmals aufgeführt werden:

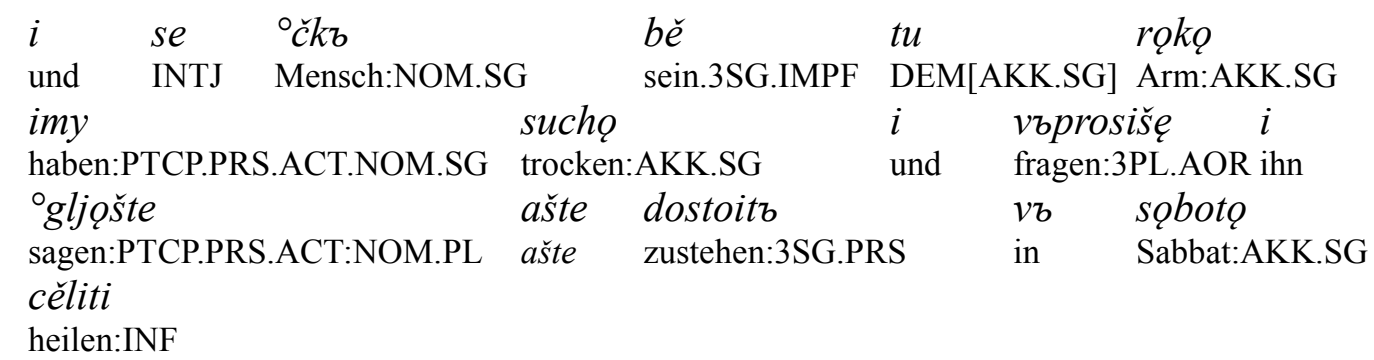

'Da war ein Mann, der einen gelähmten Arm hatte; und sie richteten die Frage an ihn: »Darf man am Sabbat heilen? «' (Mt. 12,10)

Hier ist eben dieser Kontext gegeben, der mit dem Merkmal [-Zweifel] bezeichnet werden kann: So ist nämlich aus dem weiteren Kontext dieser Belegstelle ersichtlich, dass die Sprecher der Frage sich zwar dessen bewusst sind, dass man nach den geltenden religiösen Regeln 
nicht an diesem bestimmten Tag der Woche jemand heilen darf, gleichzeitig aber wissen, dass es geschehen ist. Sie stellen die Frage an dieser Stelle mit eigener Gewissheit, um den Adressaten der Frage (= Jesus) zu einer kontradiktorischen Antwort zu zwingen. Ihre erwartete (und vor allem auch erhoffte) Antwort entspricht folglich der Bejahung des erfragten Inhaltssatzes.

Wie verhält es sich nun in Bezug auf den DcI (bzw. bloßen Infinitiv), der in 2.3. als mögliche Konstruktion aufgezeigt worden ist? Dieser ist aufgrund der Zuordnung zur Illokution der Imperativ- und Optativsätze mit dem Bindemittel $d a$ zusammenzustellen. Kann auch hier eine Opposition des Typus [ \pm Zweifel] postuliert werden? Die Terminologie scheint nicht ausreichend, um die letzte vermeintliche Opposition zu definieren. Die Satztypen sollen folglich nochmals gegenübergestellt werden: ${ }^{18}$

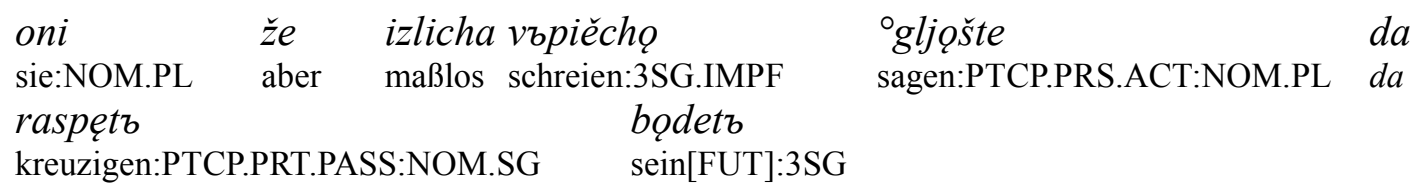

'Sie schrien nur noch lauter: »Ans Kreuz mit ihm!«' (Mt. 27,23)

$$
\begin{aligned}
& \text { azъ že }{ }^{\circ} \text { gljo vamb ne protiviti se zъlu } \\
& \text { ich aber sagen:1SG.PRS euch[DAT.PL] NEG widersetzen:INF REFL Böses:DAT.SG } \\
& \text { 'ich dagegen sage euch: Ihr sollt dem Bösen keinen Widerstand leisten' (Mt. 5,39) }
\end{aligned}
$$

In (17) liegt ein prototypischer Inhaltssatz der Imperativ- und Optativsätze vor: Sowohl die Sprecher als auch der auktoriale Schreiber gehen davon aus bzw. wissen sogar, dass dieser Aufforderung nachgekommen wird. Vermutlich liegt hierin auch der entscheidende Unterschied zu (18), wo zwar ein Gebot ausgesprochen wird und somit ebenfalls eine Illokution dieser Art vorliegt, doch nicht vorhersagbar ist, inwiefern dieses auch tatsächlich durch die Adressaten umgesetzt wird. Somit kann für diese dritte illokutive Modalität das Merkmal $[ \pm \text { real }]^{19}$ als ausschlaggebend verzeichnet werden. Bedenkt man die Eigenschaften des Merkmals [ \pm Zweifel], erscheint es nicht abwegig dieses nun mit dem Merkmal der Modalität der Imperativ- und Optativsätze zu ersetzen: Ist ein Sprecher nämlich im Zweifel über den deklarativen oder interrogativen Inhaltssatz, dann zweifelt er schlichtweg an dessen Richtigkeit, sodass hierfür im Folgenden das Merkmal [-real] vergeben werden soll. Selbiges gilt mutandis mutatis natürlich ebenso für das Merkmal [+real]. So lässt sich abschließend folgende Darstellung als Bearbeitung der Tab. 1 wiedergeben:

\begin{tabular}{|c|c|c|c|}
\hline & deklarativ & interrogativ & direktiv \\
\hline+ real & $j a k o$ & $a s ̌ t e$ & $d a$ \\
\hline -real & AcP/AcI & $j e d a$ & DcI \\
\hline
\end{tabular}

Tab. 2

Konzeptionell ist das distinktive Merkmal $[ \pm$ real] in der linguistischen Literatur bereits bekannt und regelhaft dem Felde der epistemischen Modalität zugeordnet; allerdings ist eine Unbeständigkeit in der terminologischen Benennung auffällig. Für einen ersten, (sehr) kurzen

18 Vgl. (11) und (15).

19 Vgl. nochmals Daibers (2009: 369) Hinweis zum Ausdruck der „Wahrheitsbedingungen des wiedergegebenen Redebeitrages“ durch das jako recitativum im Russisch-Kirchenslavischen. 
Einblick in deutschsprachige, slavistische Beschreibungen s. Krause (2007: 51-53), die selbst sicherlich zutreffend von „wahrscheinlichkeitsbasierter Bewertung“ spricht. Für einen knappen Einstieg in einen anglophonen, typologischen Diskurs s. Boye (2012: 133-136), der überordnend die Bezeichnung, full, partial, neutral support" nutzt.

Wichtig bei dem hiesigen Ergebnis ist das Bewusstsein, dass es sich vorerst um einen syntaktisch, semantisch ${ }^{20}$ und textuell stark beschränkten Kontext handelt: Diese Verteilung gilt bisher nur für Folgesätze zum verbum dicendi glagolati im ersten Evangelium nach Matthäus.

\subsection{Die übrigen Evangelia im Codex Zographensis}

Nachdem anhand des Sprachmaterials des ersten Evangelium nach Matthäus in der Version des Codex Zographensis die paradigmatischen Verhältnisse um das Bindemittel jako dargestellt und ihren Funktionen entsprechend systematisiert sind, sollen im folgenden Abschnitt die bisherigen Ergebnisse um das Sprachmaterial der übrigen drei Evangelia (nach Markus, Lukas und Johannes) ergänzt werden. Die Besprechung folgt hierfür den drei für grundlegend empfundenen Illokutionen: deklarativ, interrogativ und Imperativ- und Optativsätze.

\subsubsection{Die deklarative Illokution}

Im Bereich der deklarativen Inhaltssätze finden sich im Gegensatz zum ersten Evangelium, in welchem dieses Phänomen bestenfalls in syntaktisch ambigen Konstruktionen vermutet werden kann, zahlreiche Beispiele syntaktisch eindeutiger Fälle des sogenannten jako recitativum:

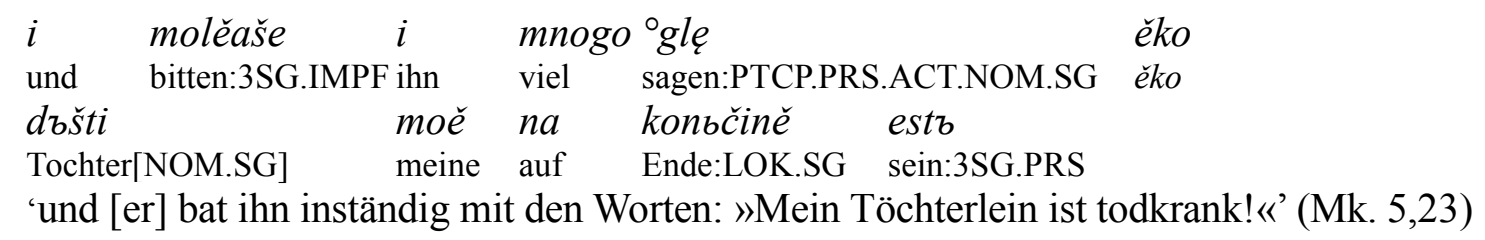

Da nun syntaktisch disambiguierte Fälle zu eindeutigen Belegen eines jako recitativum führen, stellt sich unvermeidlich die Frage, inwiefern die entsprechenden Folgesätze eine zusätzliche Semantik durch das Bindemittel erhalten. Daiber (2013: 115) spricht sich in diesem Kontext bezüglich des Bindemittels jako für eine „modale Funktion, nämlich die Anzeige der Übernahme der Illokution der Redewiedergabe seitens des Wiedergebenden“" aus. Interessant ist nun hierbei, dass in der Großzahl der Fälle nach dem hier untersuchten verbum dicendi der Inhaltssatz der direkten Rede eine deklarative Illokution enthält, was durch die Semantik des hier untersuchten verbum dicendi bedingt zu sein scheint (vgl. Fußnote 15) und somit an dieser Stelle erwartet wird. Gleichzeitig kennzeichnet aber jako in einem paradigmatischen Verhältnis zu den unter 2.4. zusammengefassten syntaktischen Mitteln ebenfalls die deklarative Illokution. Folglich ist in einer Vielzahl der hier untersuchten Belegstellen eine gewisse Redundanz der Markierung [+deklarativ] für den entsprechenden Inhaltssatz zu erwarten. Diese Redundanz scheint nach dem untersuchten Material eine emphatische Funktion zu kennzeichnen: ${ }^{21}$

\footnotetext{
20 Vor allem die Semantik des Verbums ist für den Folgesatz entscheidend, da es lexikalisch bedingte illokutive Modalitäten erwarten lässt.

21 Vgl. hierzu ebenso die vorangehende Belegstelle (19).
} 


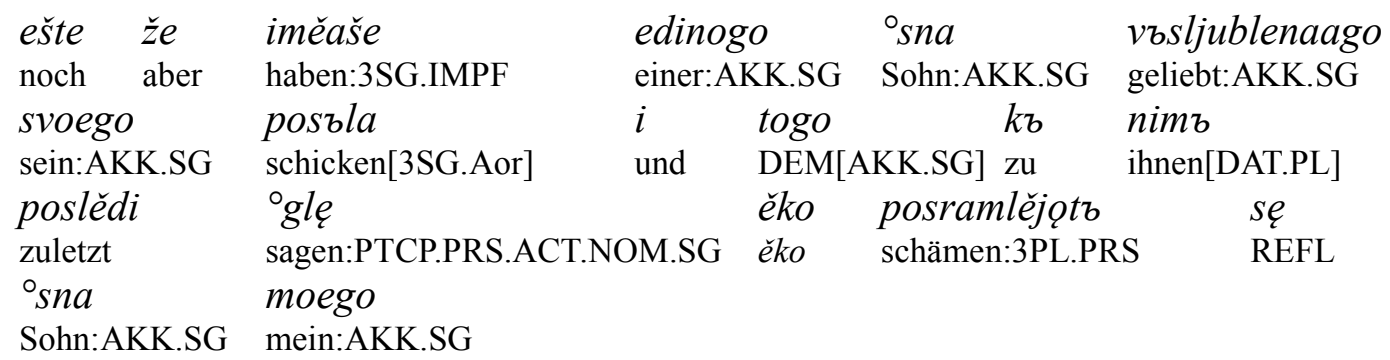

'Nun hatte er noch einen einzigen, seinen geliebten Sohn; den sandte er zuletzt auch noch zu ihnen, weil er dachte: Sie werden sich doch vor meinem Sohne scheuen.' (Mk. 12,6)

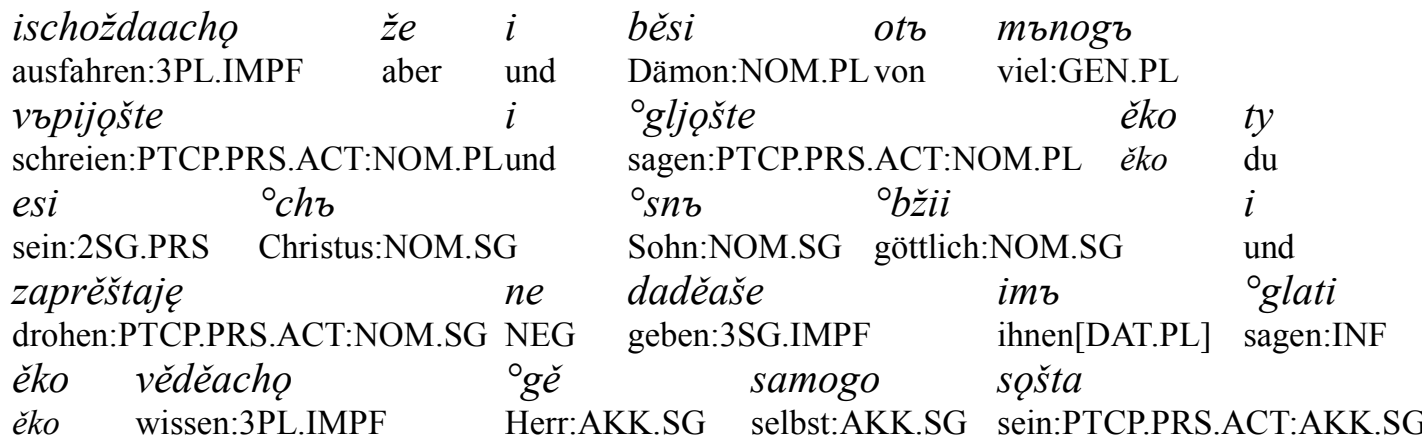

'Auch böse Geister fuhren von vielen aus, wobei sie laut schrien und ausriefen: »Du bist der Sohn Gottes! « Er bedrohte sie jedoch und ließ sie nicht zu Worte kommen; denn sie wußten, daß er Christus war.' (Lk. 4,41)

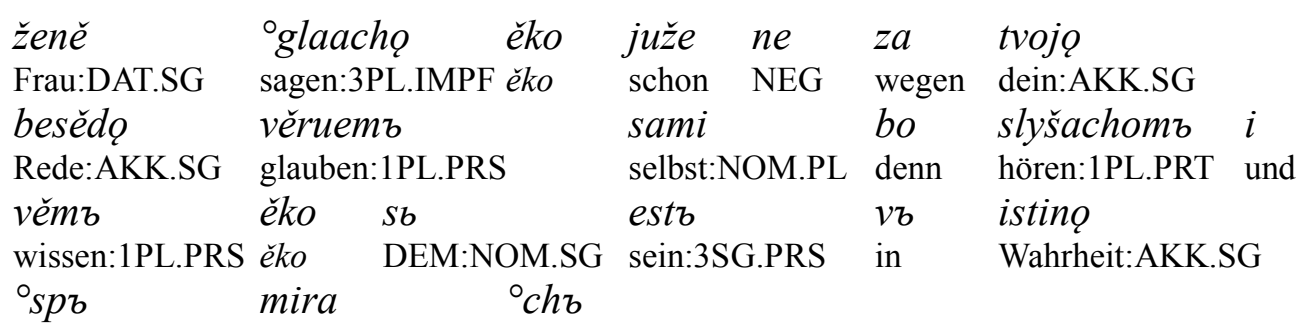

Retter:NOM.SG Welt:GEN.SG Christus:NOM.SG

'und [sie] sagten zu der Frau: »Wir glauben jetzt nicht mehr infolge deiner Aussage; denn wir haben nunmehr selbst gehört und wissen, daß dieser wirklich der Retter der Welt ist.«' (Jh. 4,42)

In allen angeführten Belegen ist eindeutig ersichtlich, dass der jeweilige (ursprüngliche) Sprecher der wiedergegebenen Rede in besonderem Maße von dem Wahrheitsgehalt seiner Aussage überzeugt ist: In (20) entsendet ein Weinbergbesitzer seinen einzigen Sohn zu den Pächtern seines Gutes, die allen zuvor gesandten Dienern gegenüber gewalttätig sind, weil er ü berze u gt ist, dass sie seinem eigenen Erben nichts antun werden. In (21) rufen Dämonen Jesus zu, dass er der Sohn Gottes sei, weil sie seine Gestalt erkannt haben. Und in (22) betonen die Samariter, dass sie nicht mehr wegen des Zeugnisses einer Dritten an die Wunder glauben, weil sie nun selbst den Beweis erlebt haben. Die Realisierung dieses betonten Wahrheitsgehaltes aus den angegebenen Belegstellen wird schließlich durch den auktorialen Erzähler mit der an dieser Stelle als Marker der realen deklarativen Illokution redundanten, aber dadurch emphatisch wirkenden Partikel jako gekennzeichnet, was zum Auftreten des vorerst unerwarteten recitativum führt und eine scheinbare oratio semirecta erzeugt. 
Hinsichtlich des AcI (bzw. AcP) zum Ausdruck der deklarativen Illokution mit Zweifeln seitens des Sprechers finden sich weitere zwei Belege, die als Untermauerung der Darstellung in Tab. 2 herangezogen werden können:

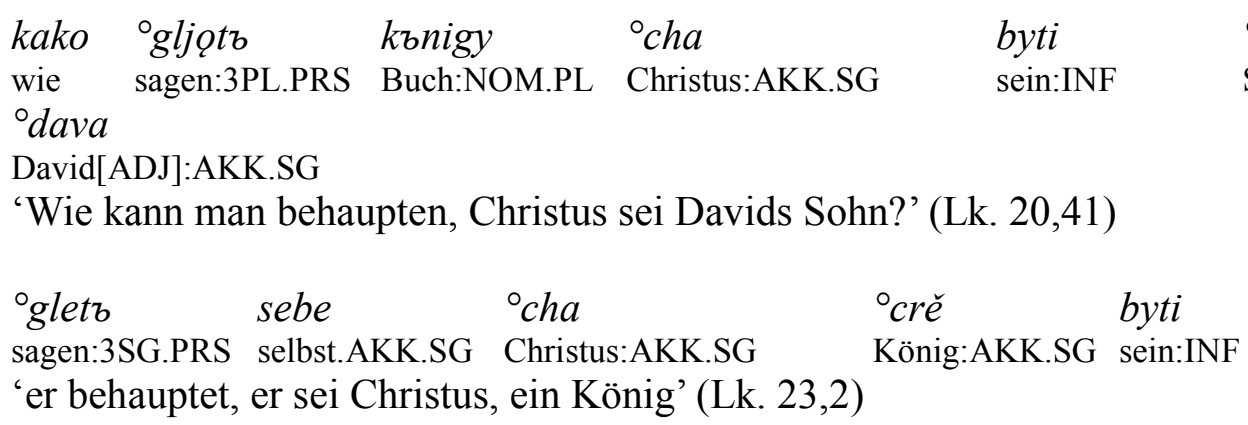

In (23) spricht Jesus selbst und ist somit über den Wahrheitsgehalt der Aussage in Zweifel, da er weiß, dass die Aussage nicht der Realität entspricht. Ähnlich stellt in (24) die Aussage Teil einer Anklage dar, sodass auch hier die Sprecher die Unrichtigkeit der Aussage unterstreichen möchten. Folglich tritt in beiden Belegen das Merkmal [-real], wie erwartet, auf.

\subsubsection{Die interrogative Illokution}

Nicht nur im Evangelium nach Matthäus, sondern auch im übrigen Corpus ist in der hier untersuchten Stellung (nach dem frequentesten verbum dicendi) das Auftreten interrogativer Bindemittel marginal. Diese Distributionsverhältnisse werden parallel zu den Bemerkungen der Illokution der Imperativ- und Optativsätze auch bei der interrogativen Illokution an der Semantik des Verbums glagolati liegen. Für das Bindemittel ašte findet sich kein weiterer Beleg, doch das Bindemittel jeda übernimmt in allen weiteren Belegen ebenfalls die oben (s. 2.4.) besprochene Funktion des Zweifels am Wahrheitsgehalt des Inhaltssatzes der interrogativen Illokution: ${ }^{22}$

$\begin{array}{llllll}\text { 'glacho } \quad \check{z} e & \text { ijudei } & \text { eda } & \text { se } & \text { samb } & \text { ubietb } \\ \text { sagen:3PL.IMPF aber } & \text { Jude:NOM.PL } & \text { jeda } & \text { REFL } & \text { selbst:NOM.SG töten:3SG.PRS } \\ \text { 'Da sagten die Juden: } & \text { »Will er sich etwa das Leben nehmen? «' (Jh. 8,22) }\end{array}$

\subsubsection{Die Illokution der Imperativ- und Optativsätze}

Wie bereits in 2.4. dargestellt, verfügt das Aksl. über diverse Mittel des Ausdruckes der Illokution der Imperativ- und Optativsätze in Folge des hier untersuchten verbum dicendi: Entweder wird der folgende Inhaltssatz mit dem Bindemittel $d a$ und einem Verbum im finiten Indikativ realisiert oder es tritt kein Bindemittel auf und die Verbmorphologie entspricht dem Infinitiv. Da das logische Subjekt bei tatsächlicher Realisierung im Dativ erscheint, kann man an dieser Stelle von einem DcI sprechen. Die bisherige Erklärung zu dieser formalen Opposition liegt im Merkmal $[ \pm$ real].

Betrachtet man nun die weiteren Belege zu der ersten Konstruktion (17) mit dem Merkmal [+real], werden die bisherigen Annahmen nicht widerlegt, bedürfen jedoch stellenweise der Interpretation:

22 Vgl. neben der folgenden Belegstelle: Jh. 7,41, Jh. 18,17 und Jh. 21,5. 


$\begin{array}{lllll}n b \quad s i & { }^{\circ} g l j Q & d a & v y & { }^{\circ} \text { speni } \\ \text { sondern DEM:AKK.PL } & \text { sagen:1SG.PRS } & d a & \text { ihr[NOM.PL] } & \text { retten:PTCP.PRT.PASS:NOM.PL }\end{array}$
bodete $\operatorname{sein}[\mathrm{FUT}]: 2 \mathrm{PL}$

'sondern [ich] erwähne dies nur deshalb, damit ihr gerettet werdet' (Jh. 5,34)

$\begin{array}{llllll}\text { si } & \text { oglachb vamb } d a & \text { ne } & \text { sbblaznite } & \text { se } \\ \text { DEM:AKK.PL } & \text { sagen:PRT.1SG euch } d a & \text { NEG } & \text { Anstoß.nehmen:3SG.PRS REFL } \\ \text { 'dies habe ich euch gesagt, damit ihr nicht } & \text { Anstoß nehmt' (Jh. 16,1) }\end{array}$

Die Belege werden durch Finalsätze ins Deutsche übertragen, können aber ebenso mit „dass ihr gerettet werden möget" und „dass ihr nicht Anstoß nehmen möget" wiedergegeben werden. Wichtig ist vor allem die Annahme, dass der Sprecher ( $=$ in beiden Fällen Jesus) davon ausgeht, dass diese Aufforderung der tatsächlichen zukünftigen Realität entspricht.

Gibt es Zweifel gegenüber dem Wahrheitsgehalt der Aussage, erscheint dementsprechend in den altkirchenslavischen Belegstellen in diesem Kontext nicht die obige Konstruktion, sondern der DcI: ${ }^{23}$

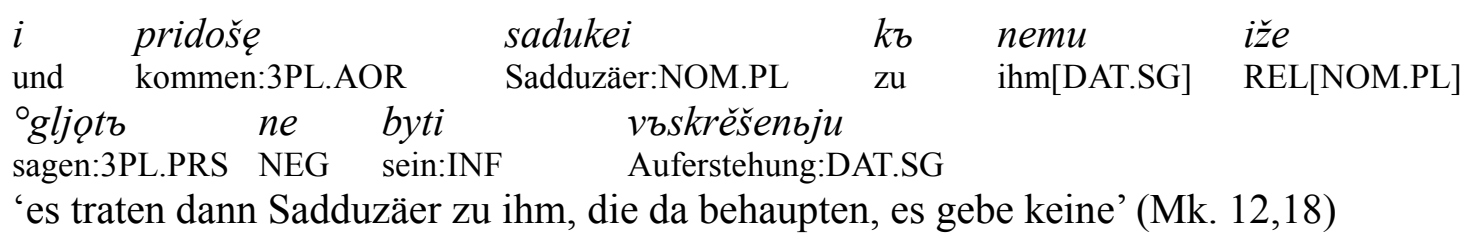

Dieser Beleg ist der hiesigen Übersetzung folgend sicherlich der deklarativen Illokution zuzuordnen oder dieser zumindest nahe. Allerdings ist auch eine Interpretation möglich als „sie sprachen, dass es keine Auferstehung gebe", insofern diese Sprecher ihrem eigenen Glaubenskanon entsprechend gegen den Glauben an die Auferstehung predigen. Dies ist nicht nur im weiteren Kontext an ihrer Frage an Jesus erkenntlich, die diesen mit einem logischen Problemfall in Zusammenhang mit der Auferstehung konfrontieren soll (s. dort), sondern entspricht auch dem historischen Wissen über die Philosophie der Sadduzäer (vgl. Stemberger 1991: 68-70). Somit scheinen die hier beschriebenen Distributionsverhältnisse den altkirchenslavischen syntaktischen Mitteln aus dem übrigen Codex der Darstellung aus 2.4. zu entsprechen und bekräftigen diese folglich.

\section{Conclusio}

Ausgangspunkt der vorliegenden Arbeit stellt das sogenannte jako recitativum dar, welches in der Forschungsliteratur zum Altkirchenslavischen vor allem aus syntaktischer Sicht einen Sonderstatus erhalten hat, insofern hierfür der Terminus der oratio semirecta nach Večerka (2002: 421) eingeführt worden ist. In den Arbeiten Daibers (2008, 2009, 2013) und Perelmutters (2009) ist das Auftreten dieses jako recitativum im Altkirchenslavischen, RussischKirchenslavischen und Altrussischen verstärkt auf seine Funktionen hin untersucht worden, wobei sowohl die Modalität (i.w.S.) als auch die Pragmatik als wichtige Beschreibungsfelder für dieses Phänomen hervortreten.

23 Vgl. Lk. 20,27. 
Der vorliegende Beitrag hat ausgehend von über 900 Folgesätzen zum verbum dicendi glagolati das jako recitativum in Hinsicht auf seinen paradigmatischen Austausch mit übrigen Mitteln der altkirchenslavischen Syntax aus einer breiteren Perspektive untersucht. Die Grundannahme Večerkas (2002: 399), dass die Mittel der altkirchenslavischen Syntax in ihren Distributionsverhältnissen einer dreigliedrigen Kategorisierung innerhalb der illokutiven Modalität folgen, hat sich bestätigt. Zudem konnte nach erfolgter Datenauswertung das Merkmal [ \pm real] als Urteil über die „Wahrheitsbedingungen“ des jeweiligen Inhaltssatzes aus Perspektive des Sprechers ergänzt werden, um die Verteilung der untersuchten sprachlichen Mittel der altkirchenslavischen Syntax präziser zu beschreiben. Hierbei bleiben allerdings weitere Bearbeitungsfelder offen, die sicherlich der Diskussion bedürfen:

An erster Stelle muss eine methodologische Diskussion erfolgen, die ihren Fokus auf die Spezifika historischer Textzeugen legt, wobei vor allem die syntaktischen Analysemöglichkeiten erweitert werden müssen. In der hiesigen Arbeit hat sich am Beispiel des frequentesten verbum dicendi gezeigt, dass die untersuchten Konstruktionen syntaktisch oftmals ambig sind. Eindeutige hypotaktische Konstruktionen bleiben in diesem Kontext sogar gänzlich aus. Wie in bisherigen Arbeiten geschehen ist an dieser Stelle ebenfalls das Kriterium der Personaldeixis angewandt worden, ohne aber ambige Konstruktionen mal als hypotaktische Objektsätze, mal als parataktische Konstruktionen mit jako recitativum zu kategorisieren. Diese stellenweise willkürliche Kategorisierung muss auch im weiteren Vorgehen neben der Beurteilung nach der Personaldeixis durch andere Kriterien ergänzt werden. Naheliegend ist hierbei eine Untersuchung der Transpositionsverhältnisse der Lokal- und Temporaldeixis. Doch auch weitere Kriterien der Erkennung hypotaktischer Konstruktionen, wie beispielsweise die Zuweisung der Illokution durch den übergeordneten Satz oder, wo im Textzeugen ersichtlich, suprasegmentale Eigenschaften, müssen systematisch erfasst und für historische Textzeugen erprobt werden.

Des Weiteren ist nicht nur hinsichtlich der Syntax eine eingehende Betrachtung der Verhältnisse in den Modellsprachen, Griechisch und Latein, notwendig. Auf syntaktischer Ebene können die Vorlagen der altslavischen Übersetzungstexte gegebenenfalls zur Disambiguierung beitragen, doch auch hier müssen die Kriterien zur syntaktischen Analyse theoretischen Maßstäben genügen; ein intuitives Sprachgefühl der klassischen Altertumssprachen oder deren traditionelle Grammatikschreibung sind sicherlich keine hinreichenden Kriterien. Letztlich ist selbst die Annahme problematisch, die sich hinter der Disambiguierung durch die Vorlageverhältnisse im Bereich der Syntax verbirgt. Ist bspw. wirklich anzunehmen, dass eine Hypotaxe im Lateinischen - durch einen AcI - ein jako in der Übersetzung als eindeutigen Komplementierer bestimmt? War dem jeweiligen Übersetzer diese syntaktische Kategorisierung bewusst oder erfolgt sie intuitiv? Schließlich kann vor allem das Griechische mit den Distributionsverhältnissen des ö $\tau$ (recitativum) und anderer syntaktischer Mittel über Wiedergabemuster im Altkirchenslavischen Aufschluss geben. Doch auch eine breitere areale Perspektive sollte angedacht werden, wobei vor allem das Bibelhebräische und das Altsyrische eine Rolle spielen müssen. ${ }^{24}$

Letztlich ist am Material der vorliegenden Untersuchung zu erkennen, dass die illokutive Modalität ein wichtiger Bestandteil der Beschreibungsmöglichkeiten der altkirchenslavischen Syntax darstellt. Hierbei weist das vorliegende verbum dicendi eine gewisse Affinität für das Auftreten deklarativer Folgesätze auf, womit der Verbsemantik offensichtlich ebenfalls eine Rolle in der Verteilung der beschriebenen Mittel zukommt. Interessant ist in einem nächsten Schritt eine Gegenüberstellung der Folgesätze zu diversen verba dicendi gegenüber verba

24 Einen ersten, kritischen Einblick zu einem potentiellen bibelhebräischen recitativum liefert Zorell (1933). 
dubitandi (mit einer möglichen Affinität zur interrogativen Illokution) und verba postulandi (mit einer möglichen Affinität zur Illokution der Imperativ- und Optativsätze). Im Allgemeinen soll an Daibers (2009: 384) abschließende Anmerkung hinsichtlich des jako recitativum erinnert werden, dass „,dessen Erforschung offenbar zu wiederholten Malen in der Wissenschaft angeregt werden muss.“

\section{Literatur}

Boye, K. (2012): Epistemic Meaning - A Crosslinguistic and Functional-Cognitive Study. Berlin (= Empirical Approaches to Language Typology, 43).

Daiber, Th. (2008): Inhaltssätze mit eže oder jako in der Vita des Makarij Koljazinskij (VMČ März, fol. 401a-408d). In: Junghanns, U. (Hg.), Linguistische Beiträge zur Slavistik: XIII. JungslavistInnen-Treffen in Leipzig, 23. - 26. September 2004. München (= Specimina Philologiae Slavicae, 149). 29-48.

Daiber, Th. (2009): Direkte Rede im Russisch-Kirchenslavischen. In: Besters-Dilger, J. \& Rabus, A. (Hgg.), Text - Sprache - Grammatik - Slavisches Schriftum der Vormoderne (Festschrift für Eckhard Weiher). München \& Berlin (=Die Welt der Slaven Sammelbände, 39). 363-386.

Daiber, Th. (2013): Modalität der Redewiedergabe - яко (recitativum) im Codex Marianus. In: Kempgen, S. et al. (Hgg.), Deutsche Beiträge zum 15. Internationalen Slavistenkongress Minsk 2013. München et al. (= Die Welt der Slaven Sammelbände, 50). 109-116.

Graf, E. (2011): Interjektionen im Russischen als interaktive Einheiten. Frankfurt am Main et al. (= Berliner slawistische Arbeiten, 38).

Krause, M. (2007): Epistemische Modalität-Zur Interaktion lexikalischer und prosodischer Marker. Dargestellt am Beispiel des Russischen und des Bosnisch-Kroatisch-Serbischen. Wiesbaden (= Slavistische Studienbücher. Neue Folge, 17).

Perelmutter, R. (2009): Pragmatic functions of reported speech with jako in the Old Russian Primary Chronicle. Journal of Historical Pragmatics 10/1. 108-131.

Schaeken, J. \& Birnbaum, H. (1999): Die altkirchenslavische Schriftkultur - Geschichte - Laute und Schriftzeichen - Sprachdenkmäler (mit Textproben, Glossar und Flexionsmustern) - Altkirchenslavische Studien II. München (= Slavistische Beiträge, 382).

Sonnenhauser, B. \& Noel, P. (2013): Vocative! In: Sonnenhauser, B. \& Noel, P. (Hgg.), Vocative. Berlin et al. (= Trends in linguistics, 261). 1-24.

Večerka, R. (2002): Altkirchenslavische (Altbulgarische) Syntax - IV. Die Satztypen: Der zusammengesetzte Satz. Freiburg i. Br. (= Monumenta Linguae Slavicae Dialecti Veteris, 46).

Vondrák, W. (1928): Vergleichende slavische Grammatik - II. Band - Formenlehre und Syntax, neubearbeitet von Grünenthal, O. Göttingen.

Zorell, F. (1933): Gibt es im Hebräischen ein «kî recitativum»? Biblica, vol. 14, issue 4. 465-469. 


\title{
Das Verständnis von Vulgärlatein in der Renaissance im Rahmen der Diskussion um das vulgare der römischen Antike vor dem Hintergrund der questione della lingua
}

\author{
Roger Schöntag (Erlangen)
}

\begin{abstract}
Während in der mittelalterlichen Vorstellung, wie sie auch bei Dante repräsentiert ist, das Lateinische als eine unveränderliche Sprache in Zeit und Raum gilt, die im Gegensatz zu den Volkssprachen durch eine Grammatik fixiert ist, beginnt in der Renaissance im Zuge eines Interesses an der Fragestellung, welche Sprache in der römischen Antike wohl gesprochen wurde, ein Verständnis für die Variabilität des Lateinischen als lebendige Sprache. Die vorliegende Untersuchung zeichnet den Erkenntnisweg der humanistischen Gelehrten zwischen 1435 und 1601 nach und gibt dabei einen Einblick in die mitunter polemische Debatte jener Epoche, an deren Ende sich der Blick auf das Lateinische dahingehend verändert hat, dass nach und nach einige grundlegende Prozesse des Sprachwandels und Aspekte der diasystematischen Heterogenität der lateinischen Sprache zu einer communis opinio geworden sind.
\end{abstract}

\section{Einleitung}

Die vorliegende Untersuchung zum sich langsam entwickelnden Verständnis von der Diversität und Heterogenität des Lateinischen als natürlicher Sprache der Antike und dem sich dabei herauskristallisierenden Begriff und Konzept des 'Vulgärlateinischen' ist im Kontext einer sprachtheoretischen Diskussion in Italien $\mathrm{zu}$ sehen, die man questione della lingua ('Sprachenfrage') genannt hat, weil ihr zentraler Gegenstand die Frage oder genauer die Suche nach der geeigneten Sprache ist. Sprache meint hier zunächst einmal vor allem Literatursprache, d.h. Schriftsprache, später dann auch allgemeine Standardsprache, d.h. sowohl die schriftliche als auch die mündliche Kommunikation betreffend. Die Tatsache, dass die Sprache geeignet sein soll, referiert in diesem Zusammenhang auf das ausreichende Prestige bzw. auf die Adäquatheit in Bezug auf literarische Zwecke - oder anders ausgedrückt, die Sprache (bzw. Varietät) soll der ,hohen“ Literatur würdig sein.

Diese Diskussion um die Frage, welche Varietät in der Literatur Verwendung finden sollte und letztlich die Grundlage für einen nachmaligen überregionalen Standard bilden wird, ist eine spezifisch italienische, die in anderen Ländern der Romania nicht in diesem Maße virulent war. Wie in ganz Europa war in Italien über die Antike hinaus und das ganze Mittelalter hindurch die prestigeträchtige Schriftsprache, d.h. die high variety, das Lateinische. ${ }^{1}$ Im Gegensatz zu Frankreich, Spanien und Portugal, wo sich recht früh politische und

1 Das Griechische galt zwar ebenfalls als Prestigesprache, doch waren Griechischkenntnisse während des gesamten Mittelalters unter den Gelehrten Westeuropas (ausserhalb des byzantinischen Einflusses) wenig verbreitet. Roger Bacon (ca. 1214/1220-1292) war einer der ersten, der zur besseren Exegese der Bibel und der antiken philosophischen Texte zur Erlernung des Griechischen (und Hebräischen) aufforderte (cf. Opus maius; Compendium studii philosophiae) und deshalb auch je eine Grammatik dazu schrieb (Grammatica graeca; Grammatica hebraica); aber erst mit der Renaissance gewann das Griechische wieder an Bedeutung. 
kulturelle Zentren etablierten, deren Varietät zur voll funktionsfähigen Standardsprache ausgebaut wurde und sich neben dem Lateinischen als Schriftsprache etablierte, war die Besonderheit in Italien zum einen die politische Zersplitterung und damit einhergehend das Fehlen eines kulturellen Zentrums bzw. die Existenz mehrerer lokalen Zentren (cf. Sizilien: scuola siciliana 'sizilianische Dichterschule', Toskana: dolce stile nuovo 'der süsse neue Stil', Piemont: piemontesische koiné, Republik Venedig: veneziano, Norditalien: franco-italiano, etc.), aber auch die Nähe der italienischen Volkssprache zum Latein, die noch lange eine gegenseitige Verständlichkeit gewährleistete und so die frühe Notwendigkeit der Verschriftung und Verschriftlichung der Volkssprache verzögerte.

Was den zeitlichen Rahmen der questione della lingua angeht, so gehen die Meinungen in der Forschung z.T. erheblich auseinander. Dennoch kann man so etwas wie eine maximale Ausdehnung postulieren, sozusagen eine questione im weitesten Sinne, die dann als Eckdaten zum einen Dante Alighieris (1265-1321) Traktat De vulgari eloquentia (1304-1307/8) hätte, in der eben jene Suche nach der idealen Literatursprache Italiens, dem vulgare illustre thematisiert und zum anderen die zweite bzw. dritte Fassung von Alessandro Manzonis (1785-1873) I Promessi sposi (1840-1842). Die questione im engeren Sinn wiederum beschränkt sich auf die Auseinandersetzung zwischen den Gelehrten des 15. und 16. Jh., eine metasprachliche Diskussion im Rahmen des Humanismus und der Renaissance-Kultur, die sowohl mündlich als auch schriftlich geführt wurde, überliefert in zahlreichen Traktaten mit expliziten Stellungnahmen. ${ }^{2}$

In der vorliegenden Darstellung soll es jedoch nicht um diese „klassische“ questione, d.h. der questione della lingua im engsten Sinne gehen, sondern um einen Aspekt der zur questione im weitesten Sinne gehört, bzw. wenn man ihn nicht dazu zählt, als eigenständiger Disput parallel dazu verläuft. Dabei handelt es sich um die grundsätzliche Frage, welche Art von Latein in der Antike gesprochen (und geschrieben) wurde.

Wie kam es zu dieser Fragestellung? Eine zweifelsfreie Antwort ist dabei nicht möglich, doch kann man als wesentliche Grundursachen die allgemeine Hinwendung zur Antike, das Aufkommen der Geschichtswissenschaft, die Begeisterung für alte Schriften und Manuskripte und eben auch das Interesse bzw. den grundsätzlichen Konflikt oder das Ringen um eine adäquate Sprache ausmachen.

Historisch verbürgt ist ein mündlicher Disput unter den päpstlichen Sekretären im Vorzimmer des Papstes Eugen IV. (1431-1447) im Jahre 1435, in dem genau diese Fragestellung diskutiert wurde (cf. Coseriu \& Meisterfeld 2003: 149-159). Im Zuge der folgenden Jahrzehnte erscheinen in loser Reihenfolge Traktate bzw. Stellungsnahmen im Rahmen diverser Schriften zu diesem Sujet, welches sich nach und nach als eigenständiges Thema in der humanistischen Gelehrtenwelt Italiens verfestigt.

Ziel der vorliegenden Zusammenschau ist es nun einen ersten Überblick mit einigen der zentralen Protagonisten über den keineswegs linearen Verlauf dieser Diskussion um die Sprache der Antike zu geben, die über fast zweihundert Jahre virulent war (bis zu Beginn des 17. Jh.), bevor sich die nach und nach herausbildende Anschauung von der Heterogenität des Lateins und dem sogenannten Vulgärlatein als Basis für die romanischen Sprachen durchsetzte.

Die erste Vorlesung zum Griechischen wurde 1397 in Florenz von Manuel Chrysoloras (1353-1415) gehalten; ausserhalb Italiens förderten u.a. Erasmus von Rotterdam (1466/1469-1536), Johann Reuchlin (1455-1522) oder Guillaume Budé (1468-1540) das Griechische.

$2 \mathrm{Zu}$ einer Periodisierung der questione della lingua und ihren verschiedenen Phasen cf. Koch (1988: 346, 357); zu einer allgemeinen Übersicht zur italienischen Sprachenfrage cf. u.a. Vitale (1967), Michel (2005: 358-408), Migliorini (2007: 309-328), Reutner \& Schwarze (2011: 117-126), Marazzini (1993, 2013). 


\section{Die Methode: Rekontextualisierung vs. sozio- und varietätenlinguistische Ver- ortung}

Um die Vorstellung der Architektur des antiken Lateins bei den italienischen RenaissanceGelehrten anhand ihrer Schriften möglichst deutlich herauspräparieren zu können und die in diesem Zeitraum sich vollziehende ideengeschichtliche Entwicklung aufzuzeigen, sollen zwei ineinandergreifende methodische Verfahren angewandt werden: Zum einen soll die zeitgenössische Traktatsliteratur ganz bewusst anachronistisch mit Hilfe des heutigen Instrumentariums sozio- und varietätenlinguistischer Begrifflichkeit analysiert werden (z.B. Diasystematik, cf. Coseriu 1973, Koch \& Oesterreicher 2011; Diglossie, cf. Ferguson 1959, Fishman 1965, 1967; Ausbaugrad, cf. Kloss 1978; allgemein, cf. z.B. Veith 2012, Sinner 2014) und somit unter dem Blickwinkel betrachtet werden, was an „Modernem“ bereits dort erkennbar ist bzw. welche Gedanken in jenen Texten bereits greifbar werden, die zu dem heutigen Erkenntnisstand führten. Zum anderen ist es nötig dazu kontrastiv die einzelnen Traktate der Gelehrten zu „rekontextualisieren“ (cf. Fleischman 1990; Oesterreicher 1998), ${ }^{3}$ also in dem zeitgeschichtlichen Diskurs zu verorten (Renaissance, Humanismus, questione della lingua), d.h. die Texte ganz traditionell philologisch, hermeneutisch $\mathrm{zu}$ interpretieren. Beide Vorgehensweisen sind dabei zwar theoretisch voneinander abzugrenzen, im konkreten Analyseverfahren der Textinterpretation und ideengeschichtlichen Rekonstruktion greifen sie jedoch ineinander, so dass deutlich wird, in welcher Weise die einzelnen Protagonisten Elemente dieser Debatte aufgreifen, verändern und gegebenenfalls weiterentwickeln.

\section{Untersuchungszeitraum und Korpus}

Die Darstellung des allmählichen Wandels dieser Vorstellungen soll mit Dante (De vulgari eloquentia, 1303/1308) als Vorläufer und Mittler zwischen mittelalterlichen und frühneuzeitlichem Wissen, der wichtige sprachtheoretische Fundamente legt, beginnen. Der eigentliche Untersuchungszeitraum konstituiert sich dann durch den Beginn des Disputes um die Sprache der antiken Römer im Jahre 1435, in welchem die beiden Traktate von Leonardo Bruni (13701444) und Flavio Biondo (1392-1463) erschienen und die, was die schriftliche Dokumentation anbelangt, als die Initiatoren der Debatte gelten können. Der Endpunkt ist durch den Trattato della vera origine (1601) von Celso Cittadini (1553-1627) gesetzt, da jener in seinem Traktat bereits eine relativ präzise und moderne Konzeption von einem latino volgare 'volkssprachlichen Latein' entwickelt hat.

Fleischman (1990: 37) verwendet erstmals den Begriff der 'Rekontextualisierung' im Rahmen der New Philology-Bewegung:

„If it is to move beyond an atomistic approach to language and to grammar, aimed simply at, filling in little holes on the great map of knowledge', then the New Philologist must, insofar as possible, recontextualize the texts as acts of communication, thereby acknowledging the extent to which linguistic structure is shaped by the pressures of discourse. It is through these and similar gestures that we might ultimately reformulate philology's role in the field of medieval studies, adapting its praxis to the challenges of postmodernism."

Oesterreicher (1998: 23), der das Verfahren der Rekontextualisierung vor allem für die Romanistik fruchtbar gemacht hat, knüpft dezidiert an die klassische Hermeneutik an:

„Den allgemein hermeneutisch zu konzipierenden Prozess dieser Rekonstruktion der verschiedenen semiotischen Bezüge der Texte durch den Betrachter bezeichne ich im folgenden als Rekontextualisierung $[\ldots] . . "$ 
Über die zeitliche Ausdehnung dieser spezifischen Diskussion herrscht in der Forschung genauso Uneinigkeit wie bei der eben geschilderten questione della lingua: So sehen Marcellino \& Ammannati (2015) den Disput als eine reine Auseinandersetzung zwischen den Initiatoren Flavio Biondo und Leonardo Bruni, Raffarin (2015) ergänzt die Zahl der Protagonisten nur um Poggio Bracciolini und Lorenzo Valla, Coseriu \& Meisterfeld (2003) sowie Tavoni (1982) lassen hingegen die Auseinandersetzung mit weiteren Autoren bis ca. Mitte des 15. Jh. gehen, Marchiò (2008) erweitert die Perspektive nur unwesentlich bis Ende des 15. Jh. Der zeitliche Rahmen und die Zahl der maßgeblich Involvierten in dieser Diskussion schwankt aus heutiger Forschungsperspektive also je nachdem wie eng oder weit man den Personenkreis fasst derer, die Wesentliches dazu beitrugen und ob es nun bei dieser Debatte um die unmittelbare erste Reaktion dieses Vorzimmergesprächs geht (v. supra) oder um weitere Auswirkungen desselben.

Nun gibt es aber gute Gründe die Diskussion über diesen ersten Höhepunkt bzw. die ersten unmittelbaren Reaktionen auf den Ursprungsstreit zwischen Bruni und Biondo weiterzuverfolgen. Nicht nur, weil auch am Ende des 15. Jh. sich noch keine Position wirklich durchgesetzt hat, sondern weil das Thema, wenn auch nicht direkt, immer wieder im Rahmen der questione aufgegriffen wird.

Die in dieser Untersuchung gewählte Perspektive auf die Debatte um die Sprache der römischen Antike erstreckt sich deshalb prinzipiell bis zu Celso Cittadini, da es auch im 16. Jh. weitere wichtige Denkanregungen zu dieser Diskussion gab, das Thema weiterhin virulent war und schließlich durch Cittadini zu Beginn des 17. Jh. nicht nur die Fragestellung explizit in einer größeren Abhandlung wiederaufgegriffen wird, sondern auch die Sprachsituation der Antike das erste Mal systematisch mit Sprachbelegen aus den verschiedensten Epochen der lateinischen Sprachgeschichte untermauert wird und die Heterogenität der lateinischen Sprache bzw. die Architektur des Lateins im heutigen Verständnis nahezu adäquat erfasst wird. Zudem wird dabei die Grundlage für den heutigen Begriff 'Vulgärlatein' gelegt und der Sprachwandel vom Latein zu den romanischen Sprachen in seinen wesentlichen Zügen dargestellt. Danach ebbt das Interesse für diese Thematik deutlich ab und rückt erst mit der Begründung der historischen Sprachwissenschaft zu Beginn des 19. Jh. wieder ins Zentrum des Interesses.

Das zugrundeliegende Korpus umfasst dabei grundsätzlich all jene Autoren und ihre Schriften, die explizit oder implizit zu dieser Debatte um die antike Sprachsituation Stellung beziehen bzw. weiterführende Gedanken dazu beitragen, wobei für eine Synopse im vorliegenden Rahmen eine Auswahl an besonders prominenten Protagonisten getroffen wurde und somit folgende Gelehrte genauer untersucht werden: Leonardo Bruni, Flavio Biondo, Leon Battista Alberti, Guarino Veronese, Poggio Bracciolini, Lorenzo Valla, Pietro Bembo und Celso Cittadini (cf. Kap. 5).

\section{Der mittelalterliche Vorläufer: Dante Alighieri}

Dante Alighieri (1265-1321) aus Florenz, berühmt vor allem durch sein Werk der Divina Commedia (1311-1321), mit dem er zum kanonischen Autor der italienischen Literaturgeschichte geworden ist und zusammen mit Francesco Petrarca (1304-1374) und Giovanni Boccaccio (1313-1375) zu den sogenannten tre corone der italienischen Literatur gerechnet wird, hat in seinem lateinischsprachigen Traktat De vulgari eloquentia (1303/1304), wiederentdeckt und übersetzt von Gian Giorgio Trissino (1478-1550) im Jahre 1529, in dem er über 
eine adäquate Literatursprache des Italienischen räsoniert, wichtige und für seine Zeit innovative Aspekte zur Sprache dargelegt. ${ }^{4}$

Im Zuge der Suche nach dem sogenannten vulgare illustre (cardinale, aulicum et curiale), ${ }^{5}$ der idealen Literatursprache Italiens, stellt Dante auch grundlegende Überlegungen zum Wesen der Sprache an sich und zum Italienischen im Speziellen an, die zu den wichtigsten Zeugnissen der frühen Sprachreflexion gehören. ${ }^{6}$ In diesen Ausführungen werden zum Teil traditionelle mittelalterliche Vorstellungen wie die Oppositionen gramatica (Latein) vs. vulgare (Volkssprache, d.h. Italienisch) oder ars vs. natura (cfr. Ellena 2011: 65) transportiert, aber auch einige sehr innovative Gedanken zur Heterogenität der Sprache (diachronische Schichtung, diatopische oder diastratische Unterschiede), die er intuitiv (vorlinguistisch) relativ präzise erfasst.

Dabei kontrastiert er die Volkssprache als die natürliche Sprache, die man von der Amme lernt (nutricem imitantes) und die ohne jegliches Regelwerk (sine omnia regula) sei, mit dem Lateinischen, welches eine sekundäre Sprache (locutio secundaria) sei, die man explizit lernen müsse und eine Grammatik habe, weswegen sie auch schlichtweg als solche bezeichnet wird (gramatica).

Sed quia unamquanque doctrina oportet non probare, sed suum aperire subiectum, ut sciatur quid sit super quod illa versatur, dicimus, celeriter actendentes, quod vulgarem locutionem appellamus eam qua infantes assuefiunt $a b$ assistentibus cum primitus distinguere voces incipiunt; vel, quod brevius dici potest, vulgarem locutionem asserimus quam sine omni regula nutricem imitantes accipimus. (Dante, DVE I, 1,2)

'Aber weil man jede Lehre nicht nur beweisen muss, sondern auch ihren Gegenstand darzustellen hat, damit klar wird, womit sie sich beschäftigt, wollen wir gleich zur Sache kommen und definieren, dass wir Volkssprache das nennen, was die Kinder von ihrer Umgebung lernen, sobald sie anfangen, Wörter zu unterscheiden; oder kürzer ausgedrückt, wenn wir ohne jede Regel die Amme nachahmen.' (Dante, DVE I, 1, 2)

Est et inde alia locutio secundaria nobis, quam Romani gramaticam vocaverunt. Hanc quidem secundariam Greci habent et alii, sed non omnes: ad habitum vero huius pauci perveniunt, quia non nisi per spatium temporis et studii assiduitatem regulamur et doctrinamur in illa. (Dante, DVE I, 1, 3)

'Wir haben auch noch eine weitere sekundäre Sprache, die von den Römern Grammatik genannt wird. Eine solche sekundäre Sprache haben auch die Griechen und andere, aber nicht alle. Nur wenige kommen dazu, sie zu beherrschen, denn man lernt und erwirbt sie nur mit großem Zeitaufwand und mit beharrlichem Studium.' (Dante, DVE I, 1, 2)

Harum quoque duarum nobilior est vulgaris: tum quia prima fuit humano generi usitata; tum quia totus orbis ipsa perfruitur, licet in diversas prolationes et vocabula sit divisa; tum quia

4 Zudem finden sich sprachtheoretische Überlegungen Dantes auch in seinem italienischsprachigen Werk Convivio (1304-1307).

5 Die Wiedergabe dieser Schlüsselbegriffe ist nicht unproblematisch, doch besteht Konsens, dass die vier Eigenschaften auf eine weite Verbreitung und dem damit verbundenen Prestige hinweisen sollen, d.h. ein Sprache die an einem (in Italien nicht existierenden zentralen) Hof sowie bei öffentlichen Anlässen gesprochen werden sollte: d.h. illustre 'erhaben, erlaucht, herausragend, edel', cardinale 'allgemein verbreitet, maßgebend, einen Dreh- und Angelpunkt bildend', aulicum 'höfisch, königlich', curiale 'höfisch, kurial, feierlich, bei öffentlichen Anlässen'.

6 Zum vulgare illustre cf. Holtus (1989), zu weiteren verschiedenen sprachtheoretischen Aspekten in De vulgari eloquentia cf. u.a. Ineichen (1973), Bossong (1990: 51-63), Wunderli (1994), Coseriu \& Meisterfeld (2003: 124-141), Marazzini (2013: 25-31). 
naturalis est nobis, cum illa potuis artificialis existat. Et de hac nobiliori nostra est intentio pertractare. (Dante, DVE, I, 4)

'Von diesen zwei Sprachen ist die Volkssprache die edlere, sowohl weil sie als erste von den Menschen gebraucht wurde, als auch, weil sie in der ganzen Welt Verwendung findet, allerdings in verschiedene Aussprachen und Wörter geteilt, und schließlich auch, weil sie für uns naturgegeben ist, während jene zweite eher künstlich ist. Die edlere Sprache von beide[n] beabsichtige ich zu behandeln.' (Dante, DVE I, 1, 2)

Weiterhin charakterisiert Dante das Lateinische als eine Sprache, die in Raum und Zeit (diversibus temporibus atque locis) unveränderlich (inalterabilis locutionis) sei (cf. Dante $D V E$ I, 9, 11). Er geht sogar so weit von den „Erfindern“ dieser grammatischen Sprache zu sprechen (inventores gramatice), um deutlich zu machen, dass es sich dabei um ein Kommunikationsmittel zur Verständigung der Gelehrten über Regionen und Länder hinweg handelt.

Das Lateinische ist demnach für Dante diachronisch invariabel, es unterliegt keinem Sprachwandel und auch eine weitere diasystematische Schichtung ist für diese Sprache ausgeschlossen, eben ganz im Gegensatz zur Volkssprache. Das Verhältnis der beiden Sprachen zueinander ist einerseits von einer gewissen Diglossie geprägt, insofern das Latein als highvariety überwiegend in einem gehobenen schriftsprachlichen Gebrauch Anwendung findet, während die Volkssprache als low-variety im Sinne einer Nähesprache verwendet wird. Allerdings ist dabei einschränkend zu vermerken, dass Dantes Ziel es ja war in diesem Traktat die Aufwertung des vulgare mit der Schaffung eines vulgare illustre zu betreiben, welches dem Latein ebenbürtig sein soll, d.h. zukünftig auch dessen distanzsprachlichen Funktionen zumindest in der Literatur übernehmen soll.

\section{Der gelehrte Disput zur Sprache der Antike seit 1435: Die einzelnen Positionen}

Die Grundproblematik, an der sich die Diskussion einst entzündete, könnte man auch auf eine einfache Frage reduzieren, nämlich: Welches Latein sprachen die Römer in der Antike? bzw. welche Varietät war die der mündlichen Kommunikation im Gegensatz zu der der schriftlichen Kommunikation?

Diese sprachhistorische und sprachtheoretische Fragestellung, deren Ursprung in einer Diskussion der päpstlichen Sekretäre lag (v. supra), ergab sich mit einer gewissen Konsequenz aus dem starken Interesse der Renaissance-Epoche für die Antike, vor allem in Bezug auf Kunst, Literatur und Architektur, der humanistischen Passion für alte Manuskripte (lat., griech.) und deren Sprache und dem damit zusammenhängenden Aufkommen der Geschichtswissenschaft bzw. historischen und antiquarischen Studien. So sind die an dieser Diskussion zunächst Beteiligten z.T. Gelehrte mit weitgefächerten Kenntnissen zur Geschichte, Literatur, Rhetorik und Philologie, die auch selbst hohe sprachliche Kompetenz (lat. Stilistik) und eine breite Textproduktion aufwiesen. 


\subsection{Leonardo Bruni (lat. Leonardus Aretinus)}

Leonardo Bruni aus Arezzo (1370-1444) vertritt in seinem Traktat An vulgus et literati eodem modo per Terentii Tuliique tempora Romae locuti sint (1435) die These, ${ }^{7}$ dass es im alten Rom zwei verschiedene Sprachen gegeben hätte, und zwar eine Sprache der Gebildeten und der Literatur, die er lingua litterata nennt und eine Sprache der Unterschicht, Illitteraten, Ungebildeten bzw. des ganzen Volkes, die er lingua vulgare nennt.

Quaestio nostra in eo consistit, quod tu apud veteres unum eumdemque fuisse sermonem omnium putas, nec alium vulgarem, alium litteratum. Ego autem, ut nunc est, sic etiam tunc distinctam fuisse vulgarem linguam a litterata existimo. (Bruni, An vulg. et lit. 2)

'Unsere Streitfrage besteht darin, dass du annimmst, dass es bei unseren Vorfahren [den Römern] eine einzige Sprechweise für alle gab, und nicht eine volkssprachliche und eine literarische. Ich hingegen glaube, dass heutzutage, wie auch damals, sich die Sprache des Volkes von der Sprache der Literatur unterschied.' [Übers. R. Schöntag]

Er begründet dies so, dass die grammatica für das einfache Volk viel zu schwierig gewesen sei, denn diese Sprache könne man wie heutzutage nur mit viel Ausbildung erlernen und richtig verstehen (Unterschiede in Bedeutung, Syntax, Flexion), die Komödien von Plautus oder Terenz hätte das Publikum nur partiell passiv verstanden und die Redner der Antike hätten ja einerseits zu anderen Gebildeten gesprochen und andererseits hinterher ihre schriftliche Fassung überarbeitet (cf. Bruni, An vulg. et lit. 5-12).

Er extrapoliert also die Zustände seiner Zeit (Diglossie mit zwei Sprachen: Italienisch bzw. italienische Dialekte vs. Latein), in der das Latein vom Volk maximal rudimentär passiv verstanden wurde, mehr oder weniger direkt auf die Zustände der Antike. ${ }^{8}$ Dabei betont er ganz in der mittelalterlichen Tradition (v. supra Dante) weiterhin die Opposition ars vs. natura bzw. grammatisch vs. ungrammatisch (bzw. regellos). Zur Entwicklung des vulgare der Antike zum vulgare seiner Zeit äussert er sich nicht explizit, noch nicht einmal, ob es überhaupt eine Entwicklung, also Sprachwandel, gab. Er argumentiert im Wesentlich aus einer Position des Lateinhumanisten, der diese Verteilung der Sprachen in der Kommunikation als gegeben ansieht und auch nicht ändern möchte, im Gegensatz zum Vulgärhumanismus. ${ }^{9}$

\subsection{Flavio Biondo (lat. Flavius Blondus)}

Flavio Biondo aus Forlí (1392-1463) hingegen vertritt in seinem Traktat De verbis romanae locutionis (1435) die Ansicht, dass es in der römischen Antike im Wesentlichen nur eine

\footnotetext{
Es handelt sich dabei um einen Antwortbrief auf das Traktat von Flavio Biondo des gleichen Jahres, welcher hier jedoch entgegen der Chronologie vorangestellt wird, da Bruni die ideengeschichtlich konservativere Position vertritt.

8 Die Tatsache, dass er mitunter von seinen Zeitgenossen (und z.T. der aktuellen Forschung) dahingehend missinterpretiert wurde, dass er glaubte die antiken Römer sprächen exakt das gleiche vulgare wie die Italiener des Quattrocento, veranlasste Marazzini (1993: 243, 245) von der tesi pseudo-bruniana zu sprechen. Tatsächlich ist anzunehmen, dass er ein nicht näher spezifiziertes „,anderes“ vulgare für die Römer annahm (cf. Valle \& Patota 2006: 69).

9 Bruni argumentiert hier zwar wie ein Lateinhumanist, in dem er durch dieses Modell einen status quo postuliert, hegt aber andererseits, im Gegensatz zu Biondo, durchaus Sympathie für das zeitgenössische volgare (in Form des Florentinischen), welches er zumindest in der Poesie (cf. Dante) als prestigeträchtig ansieht. Zu einzelnen Aspekten der Sprachauffassung Brunis cf. Coseriu \& Meisterfeld (2003: 150-153), Marchiò (2008: 29-34), Marazzini (2013: 33-39), Marcellino \& Ammannati (2015: 19-35).
} 
Sprache gab, nämlich die lateinische. Auch Biondo geht davon aus, dass es zwischen der Sprache der Gebildeten (docti, litterati, eruditi) und der des Volkes (indocti, illitterati) Unterschiede gab, aber diese seien nur graduell gewesen (cf. Biondo, De verb. rom. XV, 64). In Anlehnung an die Rhetorik (Cicero, Orator) unterscheidet er dabei drei Formen der lateinischen Sprache, und zwar die forma poetica, die forma oratoria und die forma vulgaris (cf. Biondo, De verb. rom. VIII, 39). Bemerkenswert ist in diesem Zusammenhang, dass er die Vulgärsprache durchaus als grammatikfähig ansieht (cf. Biondo, De verb. rom. XXII, 100).

Für Biondo hat sich die lateinische Sprache insbesondere die Vulgärsprache zum heutigen Italienisch entwickelt. Als Grund für den Sprachwandel sah er dabei die Korrumpierung des Lateins (corruptio) durch die Barbaren (Goten, Vandalen) an. ${ }^{10}$

Postea vero quam Urbs a Gothis et Vandalis capta inhabitarique coepta est, non unus iam aut duo infuscati, sed omnes sermone barbaro inquinati ac penitus sordidati fuerunt; sensimque factum est ut pro Romana Latinitate adulterinam hanc barbarica mixtam loquelam habeamus vulgarem. (Biondo, De verb. rom. XXV, 111)

'Aber nachdem die Stadt [Rom] erobert worden war und von den Goten und Vandalen nach und nach besiedelt wurde, waren es nicht ein oder zwei, die von der Sprache der Barbaren verschmutzt und tiefgreifend verdorben wurden, sondern alle; und allmählich geschah es dann, dass wir statt der [römischen] lateinischen Sprache diese verfälschte und mit der barbarischen vermischte Sprache hatten.' [Übers. R. Schöntag]

Biondo vollzog insofern in dieser Diskussion bzw. in Bezug auf die bisherige Vorstellung vom Wesen des Lateins einen entscheidenden Schritt, als er das gesprochene und das geschriebene Latein nicht als zwei disjunkte Sprachsysteme wahrnahm, sondern als zu einer Einheit gehörend.

Um die verschiedenen Varietäten des Lateins bzw. seine diasystematische Vielfalt zu erfassen, bedient er sich der Kategorien der antiken Rhetorik. Dabei kombiniert er diaphasische Aspekte mit diastratischen, indem er die einzelnen Register bestimmten Sprechergruppen zuordnet. Die größte Verbreitung fand jedoch seine Darstellung zum Sprachwandel, als der Veränderung des Lateins durch externe Einflüsse. ${ }^{11}$ Hierbei werden erstmalig sprachhistorisch fundierte Argumente herangezogen, ${ }^{12}$ indem Migrationsbewegungen am Ende der Antike (Völkerwanderung) für diese sprachlichen Veränderungen verantwortlich gemacht werden; ein Phänomen, welches in der modernen sprachwissenschaftlichen Forschung als Superstrattheorie beschrieben wird.

10 Zeitgenössisch ist in diesem Zusammenhang meist von corruptio ('Verderbtheit') die Rede, die aktuelle Forschung subsumiert diese Erklärung des Sprachwandels unter dem Begriff 'Korruptionsthese' oder 'Barbarenthese' (it. teoria della catastrofe) (cf. Marrazzini 1993: 259-260).

11 Zur Sprachauffassung Biondos, auch im Vergleich zu den Thesen Brunis cf. Tavoni (1984: 3-41), Coseriu \& Meisterfeld (2003: 153-158), Marchiò (2008: 17-27), Marazzini (2013: 33-39), Marcellino \& Ammannati (2015: 46-54).

12, ,...] e per la prima volta viene enunciata esplicitamente con una precisa cesura la concezione di un esisto catastrofico della latinità, che non può non coincidere con le invasioni barbariche e la caduta dell'impero romana." (Fubini 1961: 535) ' [...] und zum ersten Mal wurde mit einer präzisen Zäsur die Idee der historischen Katastrophe der Latinität explizit benannt, die unmöglich nicht mit dem Barbareneinfall und dem Fall des (römischen) Imperiums zusammenhängen konnte’ [Übers. R. Schöntag]. 


\subsection{Leon Battista Alberti (lat. Leo Baptista Alberti)}

Leon Battista Alberti (1404-1472) aus Genua legt seine Gedanken zur Sprachsituation im Vorwort zu seinem dritten Buch der Quattro libri della famiglia (1433-1440) dar. Für Alberti, der dieses Proömium kurz nach den Schriften Brunis und Biondos verfasst (1437) und sich als einziger der frühen Partizipanten des Disputs auf Italienisch äussert, gab es prinzipiell nur eine einheitliche Sprache in der Antike, was für ihn das literarische Latein war und das er als lingua commune 'Gemeinsprache' definiert.

E domanderei chi in publico o privato alcuno ragionamento mai usasse se non quella una, quale perché a tutti era commune, però in quella tutti scrivevano quanto e al popolo e tra gli amici proferiano. (Alberti, Della fam. 50-53)

'Und ich würde fragen, wer sowohl öffentlich als auch privat vernünftigerweise nicht jene eine Sprache verwenden würde, eben weil sie allen gemein ist und weil alle in ihr schreiben und sie sprechen, sowohl wenn sie sich an das Volk wenden als auch unter Freunden.' [Übers. R. Schöntag]

Diese Sprache benutzte man seiner Meinung nach zu öffentlichen wie auch privaten Zwecken und man schrieb auch in dieser Sprache. Dabei verwirft er ganz explizit die These Brunis darüber hinaus hätte es quasi eine künstliche Sprache gegeben, die nur von wenigen Gelehrten verstanden wurde (cf. Alberti, Della fam. 45). Er schließt sich also tendenziell Biondo an, weicht aber insofern von ihm ab, als er als einheitliches Latein, allein das literarische im Sinn hat. Den Sprachwandel wiederum erklärt er auch durch die Barbarenthese, d.h. die Gallier, ${ }^{13}$ Goten, Vandalen und Langobarden hätten die ehemals cultissima ed emendatissima lingua 'die kulturell hochstehenden und herausragenden Sprache' (cf. Della fam. 36-37), also das Latein, korrumpiert (con molti barbarismi e corrutela 'mit vielen Barbarismen und [anderen] Verderbtheiten', ${ }^{14}$ cf. Della fam. 34-35). ${ }^{15}$

Bei Alberti wird deutlich wie sich die Sprachenfrage um das antike vulgare und die questione della lingua sowie die gegensätzlichen Strömungen des Vulgärhumanismus und Lateinhumanismus einander bedingen. Angeregt durch den Disput zwischen Bruni und Biondo und dem allgemeinen Zweifel, ob die Volkssprache grammatikfähig sei, erarbeitet Alberti die erste Grammatik des Italienischen (Grammatichetta (vaticana), 1438/1441), ${ }^{16}$ sozusagen als vulgärhumanistisches Statement. ${ }^{17}$

13 Hier führt Alberti nun erstmalig als Verantwortliche für den Sprachwandel nicht nur Superstratvölker, sondern auch ein Substratvolk an.

14 Selbst Alberti als Vulgärhumanist geht es hauptsächlich um den Niedergang des Lateins als umwälzendes Ereignis in der Geschichte und als großen Verlust für die Gelehrtenwelt (cf. Faithfull 1953: 284).

15 Zur Position Albertis im Rahmen der questione della lingua cf. Passarelli (1999), Patota (1999); zu seinen Thesen bezüglich des antiken Lateins cf. Tavoni (1984: 42-72), Patota (1993: 97-98), Valle \& Patota (2006: 69), Coseriu \& Meisterfeld (2003: 160-161), Marchiò (2008: 35-39).

16 In seiner Grammatichetta verweist Alberti auf lexikalische und strukturelle Gemeinsamkeiten zwischen der lingua toscana und dem latino (cf. Passarelli 1999: 49).

17 Alberti begründet damit in gewisser Weise eine Tradition der Verteidigung der Volkssprache, die sich über ganz Europa ausbreitet: cf. Antonio de Nebrija (1441-1552), Louis Meigret (ca. 1500-1558), Claude de Seyssel (1450-1520), Sperone Speroni (1500-1588), Joachim Du Bellay (ca. 1522-1560), Fernão de Oliveira (1507-1581), Gruffyd Robert (ca. 1532-1598) (cf. Patota 1999: 57-58). Zur sprachtheoretischen Auseinandersetzung zwischen Vulgärhumanismus und Lateinhumanismus in Italien und Frankreich cf. bereits Strauss (1938) oder die grundlegende Synthese für Italien bei Klein (1957) sowie aktuell Schunck (2003). 


\subsection{Guarino Veronese (lat. Guarinus Veronensis)}

Guarino Veronese (Guarino da Verona, Guarino Guarini) (1374-1460) aus Verona vertrat in seinem Brief an den Fürsten Leonello d'Este (1407-1450) (Guarinus Veronensis III. Principi Leonello Marchioni Estensi de lingue latine differentiis, 1449) im Wesentlichen die Position Biondos, wenn auch aus einer anderen Perspektive. ${ }^{18}$ Er unterscheidet für die Antike zwei Formen der latinitas, einmal die Sprache aller Bewohner, auf dem Land wie auch in der Stadt (urbanos ac rusticos), die zwar eine Sprache ohne Regeln sprachen (sine ratione regulis), welches dennoch als Latein gelten kann (vox tamen ipsa litteralis), zum anderen gab es ausserdem die Sprache der Gelehrten (docti), d.h. eine latinitas, die zu einem späteren Zeitpunkt durch studio et arte entstand.

Latinitatem igitur duobus acceptam modis apud maiores animadverto uno quidem pro ea sermocinatione, qua priscos sine ratione regulis urbanos ac rusticos, uti solitos legimus, cum vox tamen ipsa litteralis esset; altero, qua studio et arte comparata docti posterius usi sunt. (Guarino, De ling. lat. diff. 8)

'Folglich erkenne ich zwei Arten der akzeptierten Latinität bei den Älteren, eine davon ist eben der Dialog, welchen die Alten ohne den Regeln der Vernunft in der Stadt und auf dem Land gebrauchen, wie wir gewöhnlich lesen, mit der Stimme genauso wie in der Schrift; den anderen, welcher durch Fleiss und vergleichende Kunst später nur von den Gelehrten gebraucht wird.' [Übers. R. Schöntag]

Bezüglich des Sprachwandels folgt er ebenfalls Biondos Barbarenthese, unterteilt die Latinität jedoch in vier Phasen, die er von Isidor v. Sevilla (Etymologiae) übernimmt (quadripartitum latinae locutionis 'die vier Epochen der lateinischen Sprache', cf. Guarino De ling. lat. diff. 13). Die Sprache des dritten Stadiums ist dabei diejenige mit der größten Regelhaftigkeit (recte romanam), dem größten Ausbaugrad, und dem höchsten Prestige (formosa, adulta, concinna) (cf. De ling. lat. diff. 18).

Guarinos innovativer Beitrag zum Disput um die Sprache der Antike besteht also zum einen im Hinzufügen weiterer Argumente bezüglich der Einheit des Lateinischen ${ }^{19}$ und zum anderen in der diachronischen Feingliederung, auch wenn dabei die Entwicklungsstadien noch eher statisch scheinen und der Bezug zum zeitgenössischen volgare nicht ganz eindeutig ist. $^{20}$

\subsection{Poggio Bracciolini (lat. Poggius Florentinus)}

Gian Francesco Poggio Bracciolini (1380-1459) setzt sich im dritten Teil seiner dialogischen Schrift Historia tripertita disceptativa convivalis, die den exakten Titel Utrum priscis romanis latina lingua omnibus communis fuerit, an alia quaedam doctorum virorum, alia plebis et vulgi, tertiae convivalis historiae disceptatio (1450) trägt, mit der Sprache im alten Rom und

18 Tavoni (1984: 81) weist darauf hin, dass Biondo eher als Historiker und Rhetoriker argumentiert, während Guarino die Sicht eines Grammatikers hat und dementsprechend auch seine Position mit Beispielen aus der lateinischen Grammatik untermauert (cf. auch Mazzocco 1993: 194-195; Coseriu \& Meisterfeld 2003: 162).

19 Er führt u.a. an, dass das gesamte Volk die Theateraufführungen verstand sowie offizielle Reden, und dass Frauen genauso wie Männer dazu in der Lage wären. Zudem gäbe es keine Überlieferung von volkssprachlichen Aufzeichnungen (cf. Mazzocco 1993: 54-55).

20 Zu Einzelheiten der Sprachposition Guarinos cf. Tavoni (1984: 73-104), Mazzocco (1993: 51-57), Coseriu \& Meisterfeld (2003: 161-162), Marchiò (2008: 41-45). 
der These Brunis auseinander. Diese möchte er nach eigener Aussage widerlegen und führt dazu zahlreiche Belege literarische Quellen ins Feld (z.B. Quintilian, Cicero, Varro, Livius). Für ihn gab es in der Antike prinzipiell nur eine allen Einwohnern Roms bzw. Latiums gemeinsame Sprache, nämlich das Lateinische, was sich schon allein daraus ergeben würde, dass die Sprache lingua latina hieß, d.h. nach der Landschaft Latium benannt wäre, ganz ana$\log$ zu Spanisch nach Spanien, Französisch nach Frankreich, oder Deutsch nach Deutschland.

Nam sicut linguam dicimus gallicam, hispanam, germanam, italam, qua Galli, Hispani, Germani, Itali loquuntur, item de graeca et reliquis, eodem modo latinam linguam eam fuisse oportet, quae in communi erat usu apud Latinos. (Bracciolini, Utrum pris. 14)

'Nämlich gleich wie die Sprache, die wir Gallisch, Hispanisch, Germanisch, Italisch nennen, welche von den Galliern, Hispaniern, Germanen, Italern gesprochen wird, genauso ist es bei Griechisch und anderen, in dieser Art und Weise nötig gewesen die lateinische Sprache so zu benennen, welche bei den Latinern in Gebrauch war.' [Übers. R. Schöntag]

Das überlieferte Latein war also für ihn somit eindeutig die Sprache des alten Roms, was er in der Literatur auch gut belegt findet. So führt er beispielsweise Quintilian an, der in seiner Institutio Oratoria darauf hinweist, dass künftige Redner (pueris ad artem oratoriam educandis 'Jünglinge, die zur Redekunst erzogen werden') sich eigentlich am besten in einem Umfeld mit korrektem Latein (recte loquantur latine) bewegen und sich nicht das Latein der Hausangestellten (non domestica loquela) zum Vorbild nehmen sollten (cf. Bracciolini, Utrum pris. 30-34). Daraus folgt für Bracciolini, dass einerseits das Latein eine Einheit war und es auch alle verstanden (latinus sermo et nutricibus et reliquis domesticis erat communis 'die lateinische Sprache war sowohl den Ammen, als auch den übrigen Hausangestellten gemein', cf. Utrum pris. 36), aber es doch so etwas wie eine Sprache bzw. Varietät der Gebildeten gab. Dies erkennt er daran, dass nur jene korrekt deklinieren konnten (z.B. senatus) und die Grammatik beherrschten (verbis omnes latine, sed non omnes grammatice loqui solitos) (cf. Utrum pris. 38). Grundsätzlich sprachen also alle Latein, aber nur wenige nach den Regeln der Grammatik.

Den Sprachwandel im eigentlichen Sinne thematisiert er nicht, jedoch zeigt er auf, dass sowohl die Sabiner, Etrusker, Osker oder Samniten Wörter in die lateinische Sprache gebracht hätten, als auch später die Gallier, Germanen und Afrikaner, ${ }^{21}$ so dass durch diese zahlreichen Fremdwörter (variis verbis commixtus) der Charakter des Lateins verworren wurde (confusior) (cf. Utrum. pris. 75-79). Das Ganze sieht er aber eher mechanisch, d.h. der historische Prozess der sprachlichen Veränderungen ist bei ihm nicht wirklich präsent, sondern er sieht dies er als einen Ersetzung von bestimmten sprachlichen Formen durch andere.

Im Gegensatz zu Bruni interpretiert Bracciolini keine diglossische Sprachsituation für die Antike, sondern spricht sich, unterstützt durch zahlreiche Belege in der römischen Literatur für eine Einheit der lateinischen Sprache aus (mit allgemeiner Interkomprehension zwischen den Sprechern), betont aber auch, dass nur die Varietät der Gebildeten (diaphasisch-diastratisch hoch markiert) den Regeln der Grammatik entspricht. ${ }^{22}$

21 Bracciolini erweitert hier die Zahl der Substrat- und Superstratsprachen im Vergleich zu den bisherigen Autoren stark, allerdings differenziert er nicht oder kaum in Bezug auf die jeweilige Chronologie der sprachlichen Beeinflussung dieser Völker.

$22 \mathrm{Zu}$ diesen und weiteren Details bezüglich der Sprachtheorie Bracciolinis innerhalb des Disputs um die antiken Sprachformen cf. Tavoni (1984: 105-116), Mazzocco (1993: 58-65), Coseriu \& Meisterfeld (2003: 162-165), Marchiò (2008: 48-62). 


\subsection{Lorenzo Valla (lat. Laurentius Valla)}

Lorenzo Valla (Lorenza della Valle) (1407-1457) aus Rom nimmt in seiner dialogischen Schrift Apologus II (1451-1453) zur Diskussion von 1435 Stellung bzw. möchte explizit Bracciolini widerlegen. ${ }^{23}$ Valla schließt sich in bestimmten Punkten der These Brunis an und gibt zu Bedenken, dass es nicht sein kann, dass in der Antike die Kinder (oder Ungebildeten) mühelos die schwierige Grammatik des Lateins beherrscht hätten. Andererseits sieht er die Volkssprache durchaus als eine Art Latein an, was seiner Meinung nach auch Bruni so verstand, nur eben von anderen falsch interpretiert wurde.

Ergo si grammatica non magis peritorum doctorumque est, quam imperitorum et indoctorum, sequitur id quod a principio proposueram, grammaticam fuisse etiam vulgi infantiumque sermonem. [...] Igitur quod volebam, doctorum sit pariter et indoctorum, apud priscos, latine ac grammatice loqui. (Valla, Apologus II, 141-142)

'Wenn also die grammatische Sprache nicht sehr kundig und gelehrt ist, noch unkundig und ungelehrt, folgt daraus, dass ich dies von Beginn an vorgeschlagen hatte, nämlich dass die grammatische Sprache einst die Sprache des Volkes und der Kinder gewesen ist. Folglich, was ich sagen wollte, ist, dass schon bei den Alten die Gelehrten, als auch die Ungebildeten, in gleicher Weise, Latein und die grammatische Sprache sprachen.' [Übers. R. Schöntag]

Außer im Apologus, legt er auch in seiner Streitschrift Antidotum I (1452) ausführlich dar, dass auch die zeitgenössische Sprache in Rom noch das Lateinische sei, allerdings ein sehr verändertes, ein verderbtes, denn wo sollte denn ein andere Sprache herkommen. Auch das grammatische Latein Ciceros habe sich ja so geändert, dass dieser es nicht wiedererkennen würde. Dabei insistiert er darauf, dass, im Gegensatz zu Guarino, der den Ursprung des Lateinischen auf der italienischen Halbinsel annahm, Latein rein auf Rom begrenzt sei, d.h. die romana lingua, die er mit der latina lingua gleichsetzt (non ergo lingua romanorum alia nunc est quam latina 'dann ist nun also die römische Sprache nichts anderes als die lateinische', cf. Valla, Apologus II, 118), dort entstanden sei und dort auch immer noch weiter existiert (cf. Mazzocco 1993: 78-79; Coseriu \& Meisterfeld 2003:1 70). ${ }^{24}$

Auch wenn er für die Veränderung der Volkssprache indirekt die Barbaren verantwortlich macht ([sermo] nunc depravato 'nun verdorbene [Sprache]', cf. Valla, Apologus II, 116), gilt sein Interesse nicht wirklich dem Sprachwandel. Dennoch thematisiert er nicht nur die Veränderlichkeit der Volkssprache, sondern bringt den Aspekt in die Debatte ein, dass auch das zeitgenössische (gesprochene) Latein der Gelehrten nicht mehr die gleiche Art von Sprache wie in der Antike ist.

Latine dico, ut docti loquebantur non ut indocti, aut quomodo nunc saeculum nostrum latine loquitur; quod potius dicendum est loqui semilatine ac semibarbare. (Valla, Apologus II, 184) 'Das Lateinische, so meine ich, wurde von den Gelehrten gesprochen, nicht von den Ungebildeten, und zwar nicht in einer Weise wie jetzt in unserer Zeit Latein gesprochen wird; was eher als halblateinische und halbbarbarische Sprechweise zu benennen ist.' [Übers. R. Schöntag]

23 „Unlike the treatises of Biondo, Bruni, Guarino, Poggio, and Filelfo, Apologus II results not so much from a genuine interest in the linguistic state of antiquity, but from a need to vilify Poggio's personal life and scholarship. In fact, Apologus II is part of an on-going polemic between Poggio and Valla" (Mazzocco 1993: 70). Eine konkrete Polemik wäre beispielsweise die Unterstellung Vallas, Bracciolini hätte Bruni missinterpretiert, obwohl dies nur auf Biondo zutraf (cf. Tavoni 1984: 130-131).

24 „LAU. Ego vero etiam hodie romanos loqui latine fateor. POG. At multum differenter a priscis” (Valla, Apologus II, 165). LAU [Lorenzo]. 'Ich glaube tatsächlich, dass auch die heutigen Römer Latein sprechen. POG [Poggio]. Welches sich von dem früheren Latein stark unterscheidet’ [Übers. R. Schöntag]. 
Valla argumentiert dabei grundsätzlich aus der Position des Lateinhumanisten, ${ }^{25}$ ist er doch maßgeblich für die Erneuerung der lateinischen Schriftsprache nach dem Muster der klassischen Latinität verantwortlich, die er durch sein Hauptwerk Elegantiarum linguae latinae libri sex (1471) befördert (cf. Bossong 1990: 67). ${ }^{26}$

\subsection{Pietro Bembo (lat. Petrus Bembus)}

Pietro Bembo (1470-1547) aus Venedig war in sprachtheoretischer Hinsicht vor allem durch seine Ausführungen zur questione della lingua und die Schaffung eine der ersten italienischen Grammatiken bedeutsam.

Sein Werk Prose della volgar lingua (1525), eine sprachtheoretische Abhandlung (1. Teil Sprachenfrage, 2. Teil: Metrik u. Rhetorik, 3. Teil: Grammatik), aufgebaut als platonischer Dialog (Gesprächsteilnehmer: Carlo Bembo, Ercole Strozzi, Federico Fregoso, Giuliano de‘ Medici $^{27}$ ist ein typisches Produkt der questione della lingua bzw. eines der wichtigsten Werke, die im Rahmen der italienischen Sprachenfrage geschaffen wurden. Darüber hinaus steuert Bembo im ersten, sprachtheoretischen Teil - eher en passant - einige wichtige Aspekte zur Diskussion um die antike Sprachsituation bei. Er postuliert dabei eine Verteilung der Sprache der Gelehrten und jener des Volkes, die sich an der diglossischen Aufteilung von Bruni anlehnt, allerdings mit der entscheidenden Innovation, dass bei Bembo die high-variety das Griechische ist.

Che sì come i Romani due lingue aveano, una propria e naturale, e questa era la latina, l'altra straniera, e quella era la greca, così noi due favelle possediamo altresì, l'una propria e naturale e domestica, che è la volgare, istrana e non naturale l'altra, che è la latina. (Bembo, Prose I, Cap. III) 'So wie die Römer zwei Sprachen hatten, eine ihnen eigene und natürliche, und dies war das Lateinische, sowie eine andere, fremde, und dies war das Griechische, so haben auch wir zwei Sprachen, eine uns eigene, natürliche und für den Hausgebrauch, welches die Volkssprache ist und eine andere, fremde und nicht natürliche, welches das Lateinische ist.' [Übers. R. Schöntag]

Diese Art der Diglossie (cf. Fishman 1967), die Bembo für die antike römische Gesellschaft annimmt (einheitliches Latein vs. Griechisch), ist insofern nicht ganz von der Hand zu weisen, als tatsächlich das Griechische, vor allem zu Beginn der römischen Staatswerdung, aber letztlich während der gesamten Dauer des Imperium Romanum immer eine wichtige Bildungssprache war. Allerdings ignoriert er hier in seiner Darstellung die mit der Zeit wachsende Wichtigkeit der lateinischen Schriftsprache, die nach und nach immer mehr zu einer dem Griechischen ebenbürtigen Schrift- und Literatursprache ausgebaut wird und in der distanzsprachlichen Mündlichkeit sowieso einen festen Platz inne hatte. Nichtsdestoweniger ist zu konstatieren, dass Bembo in diesem Zusammenhang den soziolinguistischen Faktor 'Prestige' sehr präzise erfasst (cf. stima, in prezzo, riverenza), der in Bezug auf die Sprachverwendung einer Varietät oder Sprache entscheidend ist.

25 Im Gegensatz zu den anderen an der Diskussion beteiligten Humanisten schreibt Valla keinerlei Werke in der Volkssprache (also auf Italienisch).

$26 \mathrm{Zu}$ einer ausführlichen Darstellung der Argumente Vallas in diesem Disput cf. Tavoni (1984: 117-169), Mazzocco (1993: 69-81), Coseriu \& Meisterfeld (2003: 169-171), Marchiò (2008: 64-90).

27 Die einzelnen Positionen der Protagonisten im Rahmen der questione della lingua: Carlo Bembo (= Pietro Bembo): archaisierendes Italienisch, d.h. tre-corone-Modell; Giuliano de' Medici: modernes Florentinisch; Federigo Fregoso: höfische koiné bzw. italienische Hofsprache; Ercole Strozzi: Latein. 
[...] che sì come a quel tempo e in que' dotti secoli era ne' romani uomini di molta maggior dignità e stima la greca lingua che la latina, così tra noi oggi molto più in prezzo sia $\mathrm{e}$ in onore $\mathrm{e}$ riverenza la latina avuta che la volgare. (Bembo, Prose I, Cap. IV)

' [...] so wie in jener Zeit und jenen gelehrten Jahrhunderten bei den Römern die griechische Sprache viel mehr Würde hatte und Wertschätzung genoss als die lateinische, so wird heutzutage bei uns dem Lateinischen mehr Wert beigemessen, Ehrerbietung erwiesen und Respekt gezollt als der Volkssprache.' [Übers. R. Schöntag]

Der Sprachwandel im eigentlichen Sinne ist für Bembo kein zentrales Thema, verankert er zwar den Beginn der Veränderung der volgar lingua in die Zeit der „Barbareninvasion“, bleibt jedoch zeitlich weitgehend unbestimmt (cf. Il quando... sapere...non si può). ${ }^{28}$

Für Bembo sind die wenigen Gedanken zur Sprache im antiken Rom nur ein Vehikel in seiner Argumentation im Rahmen der questione della lingua, insofern er die Dignität des Trecento-volgare durchsetzen möchte, d.h. es geht ihm um eine Aufwertung der Volkssprache, und zwar derjenigen, die sich bereits als Literatursprache bewährt hat (tre-coroneModell). ${ }^{29}$ Dabei stellt er sich prinzipiell für die Antike eine Einheit der lateinischen Sprache vor, die dann irgendwann in der Völkerwanderung, d.h. durch die corruptio der Barbaren zerstört wurde, woraus jedoch etwas positives Neues entstand, nämlich das Italienische. Er sieht dieses Vergehen und Werden (cf. Pflanzenmetapher bei Horaz) als etwas Grundoptimistisches, insofern er daraus ableitet, dass das Italienische irgendwann die gleiche Nobilität wie das Lateinische erreichen kann bzw. durch die Literatur Dante, Petrarca und Boccaccios bereits auf diesem Weg ist.

\subsection{Celso Cittadini (lat. Celsus Cittadinus)}

Celso Cittadini (1553-1627) aus Rom beschäftigt sich in seinem Trattato della vera origine e del processo e nome della nostra lingua (1601) ausführlich mit der Diversität der lateinischen Sprache und dem Verhältnis von Latein und Volkssprache. Er zieht als erster epigraphische Quellen und auch konsequent literarische Quelle der verschiedensten Epochen (auch der Spätantike und des Frühmittelalters) heran, um die Veränderung der lateinischen Sprache aufzuzeigen. Er erkennt den Sprachwandel vom Latein zum Italienischen (bzw. den romanischen Sprachen) und sagt auch als erster deutlich, dass es zwar einen Einfluss fremder Völker gegeben habe (un corrompimento della Latina favella, cf. Barbarenthese), aber die Veränderung des Lateins vor allem interne Gründe hatte (nostra lingua haver'altro cominciamento).

28 „E, lasciando le altri parti da canto, se la nostra volgar lingua non era a que“ tempi nata, ne“ quali la latina fiorì, quando e in che modo nacque ella? - Il quando - rispose messer Federigo - sapere appunto, che io mi creda, non si può, se non si dice che ella cominciamento pigliasse infino da quel tempo, nel quale incominciarono i Barbari ad entrare nella Italia e ad occuparla, e secondo che essi vi dimorarono e tenner piè, così ella crescesse e venisse in istato." (Bembo, Prose I, Cap. VII) 'Und, auch wenn wir die anderen Aspekte beiseitelassen, stellt sich die Frage, dass wenn unsere Volkssprache nicht in jenen Zeiten geboren wurde, in denen die lateinische Sprache in Blüte stand, wann und in welcher Weise entstand sie dann? - Das Wann antwortete Herr Federigo - genau zu wissen, das kann man meines Erachtens nicht, wenn man nicht annimmt, dass ihr Anfang in jene Zeit fällt, in der die Barbaren anfingen nach Italien einzuwandern und es zu besetzen, und weiterhin dass sie dort blieben und Fuß fassten, so dass sie daraus erwuchs und zur Blüte kam.' [Übers. R. Schöntag].

29 Zu Bembo im Rahmen der questione cf. z.B. Bossong (1990: 100-106), Marazzini (1993: 241-249), Marazzini (2013: 45-54), zur Diglossie-Vorstellung Bembos cf. Lüdtke (2005: 37). 
La maggior parte adunque di coloro, i quali della nostra lingua hanno ragionato, o ragionano perche vogliano che la lingua vulgare, laqual noi senz'arte, o regola alcuna, imitando la balia e la madre nostra apprendiamo; e con esso laqual noi tutti communalmente hoggi parte regolatamente, e parte nò favelliamo; havesse cominciamento, quando i Barbari, cioè, i Goti prima, o i Longobardi apresso tirannevolmente il dominio d'Italia occuparono, e non prima; è che ella sia un corrompimento della Latina favella: il che non esser molto vero; anzi essa nostra lingua haver'altro cominciamento, e più antico assai havuto, speriamo di fare apparir viè più che chiaro, e pianamente. (Cittadini, Tratt., 1r-v)

'Die Mehrheit also derer, die sich über unsere Sprache Gedanken gemacht haben oder darüber räsonieren, weil sie glauben machen wollen, dass die Volkssprache, welche wir ohne Kunst oder irgendeine Regel erlernen, indem wir die Amme oder unsere Mutter imitieren, und in der wir heute alle teils regelmäßig, teils nicht regelmäßig sprechen, ihren Anfang hatte, als die Barbaren, d.h. zuerst die Goten, und dann die Langobarden, das Herrschaftsgebiet Italiens besetzten, und nicht früher; und dass dies zu einer Korrumpierung der lateinischen Sprache geführt habe: dies ist jedoch nicht wahr, im Gegenteil, denn diese unsere Sprache hat einen anderen Beginn, ist sie doch viel älter, was wir hoffen mehr als deutlich und ausführlich darlegen zu können.’ [Übers. R. Schöntag]

Bei ihm findet sich letztlich die Grundlage für den dann später in der Sprachwissenschaft exakt definierten Terminus 'Vulgärlatein', und zwar insofern indem er deutlich macht, dass das Lateinische eine Einheit ist, dabei jedoch gleichzeitig eine Diversität aufweist (diachronisch und diastratisch-diaphasisch). Anders als seine Vorgänger spricht in diesem Zusammenhang jedoch nicht von zwei Sprachen, sondern von due sorte di lingua 'zwei Arten von Sprache' und deutet damit ein frühes Verständnis von Varietät an.

Hora egli è, prima che noi passiamo più oltre, da sapere; che per ogni tempo, e prima e poi, furono in Roma due sorte di lingua. (Cittadini, Tratt., 2r)

'Bevor wir zu anderen Dingen übergehen, ist es nun daran zu wissen, dass seit jeher, also sowohl früher, als auch später es in Rom zwei Arten von Sprachen gab.' [Übers. R. Schöntag]

Diese neue Vorstellung von den zwei Arten der Sprache kombiniert er dabei durch überkommenden Denkmuster von ars vs. natura und so beschreibt Cittadini das volgare als rozza e mezzo Barbara 'grob und halb barbarisch' (Tratt. 2r-v) und das regelhafte Latein der dicitori nobili und letterati, welches er pura latina nennt, als coltivata dall'arte 'durch die Kunst [der Regeln] kultiviert' (Tratt., 2v).

Bezüglich der diachronischen Entwicklung greift Cittadini wie bereits Guarino (v. supra) auf die Einteilung von Isidor v. Sevilla zurück und charakterisiert die vier Phasen der lateinischen Sprachgeschichte als Prisca, Latina, Romana, e Mista (Tratt., 1v). ${ }^{30}$ Allerdings ist er diesbezüglich weitaus ausführlicher als seine Vorgänger und belegt alle Sprachstufen mit zahlreichen Beispielen aus Inschriften und literarischen Quellen.

Der Hauptverdienst Cittadinis in dieser Debatte liegt zweifellos in seiner umfangreichen Beschäftigung mit diesem Thema aus sprachtheoretischer und historischer Perspektive, auch wenn dabei sein Engagement für eine bestimmte Position in der questione nicht ganz verdeckt bleibt. ${ }^{31}$ Dabei ist er derjenige, der aus heutiger Sicht einem adäquaten Verständnis der Architektur der lateinischen Sprache und dem, was man linguistisch unter ,Vulgärlatein“ versteht,

30 Tatsächlich ist gerade diesbezüglich jedoch eine starke Referenz Cittadinis auf Ludovico Castelvetro (15051571) nachzuweisen, von dem er das Isidor'sche Schema übernimmt (cf. Schöntag 2016: 21).

31 Cittadini ist Vulgärhumanist und in der Frage um die adäquate italienische Schrift- und Literatursprache vertritt er die Position der scuola senese (cf. Schlemmer 1983: 14). 
am nächsten kommt bzw. diesbezüglich die Grundlage für die historische Sprachwissenschaft im 19. Jh. legt. ${ }^{32}$

\section{6. $\quad$ Fazit}

Die Vorstellung von der Unveränderlichkeit und Universalität der Sprache, insbesondere des Lateins ist im Mittelalter tief verwurzelt. Bei Dante, der zum ersten Mal die Veränderlichkeit der Volkssprache, d.h. des Italienischen genauer betrachtet, bleibt dennoch der Gegensatz zwischen einer künstlichen Sprache (ars = Latein) und einer natürlichen Sprache (natura $=$ Italienisch) bestehen. Erst in der Renaissance gelingt es im Rahmen der Frage nach der antiken Sprachsituation das Lateinische als eine heterogene Sprache zu verstehen, d.h. modern ausgedrückt seine Architektur. Dabei ist es ein langsamer Erkenntnisprozess, dass das Latein als maximale Prestigesprache mit der wichtigsten Literatur des Okzidents, einst eine lebendige Sprache war, mit einer diasystematischen Vielfalt wie jede andere Sprache.

Im Zuge dieser Beschäftigung mit der antiken Sprachsituation entsteht auch ein Grundverständnis des Sprachwandels, der vom Lateinischen zu den romanischen Sprachen führt, eine genauere Vorstellung vom Unterschied zwischen gesprochener und geschriebener Sprache in der Antike sowie von einem gesprochenen Latein, vor allem in der Spätzeit, welches dann die Grundlage der zeitgenössischen romanischen Volkssprachen bildet (cf. Vulgärlatein). Dabei wird zunächst jegliche Veränderung durch eine corruptio von Seiten fremder Völker erklärt (cf. Barbarenthese bzw. Substrat- und Superstrateinfluss) bis man nach und nach auch einen internen Sprachwandel des Lateinischen annimmt.

Die hier vorgestellten Autoren und ihre Traktate bzw. Stellungnahmen repräsentieren freilich nur einen Teil der Debatte um die antike Sprachsituation, ${ }^{33}$ dennoch sind dabei die wohl wichtigsten Protagonisten vertreten. Dabei sollte deutlich werden, dass die Diskussion keineswegs im Sinne eines linearen Erkenntniszuwachses verlief, sondern aufgrund der Verknüpfung mit anderen zeitgenössischen Fragestellungen (questione della lingua, Humanismus) die Argumente entsprechend variiert wurden und meist eigentlich ein andere Zielsetzung beinhalteten. Des Weiteren sollte ebenso aufgezeigt werden, dass die Suche nach einem Erklärungsmodell für das Verhältnis Volkssprache vs. Latein in der Antike nicht mit der ersten Generation der Disputanten abgeschlossen war, wie es in der Forschung meist angenommen wird, sondern noch bis zu Beginn des 17. Jh. andauerte und durch weitere innovative Positionen bereichert wurde.

\section{Literatur}

Bossong, G. (1990): Sprachwissenschaft und Sprachphilosophie in der Romania. Von den Anfängen bis August Wilhelm Schlegel. Tübingen: Narr (= Tübinger Beiträge zur Linguistik, 339).

32 Zur Bedeutung Cittadinis in sprachtheoretischer Hinsicht cf. u.a. Schlemmer (1983), Ward (1990), Werner (1999), Pistolesi (2000), Schöntag (2016).

33 Weitere Renaissance-Gelehrte, die thematisch einige Gedanken zu dieser Debatte beisteuerten wären: Francesco Filelfo (1398-1481), Angelo Decembrio (1415-ca. 1467), Paolo Pompilio (1455-1491), Lodrisio Crivelli (gest. 1525), Baldessare Castiglione (1478-1529), Claudio Tolomei (1492-1556), Pier Francesco Giambullari (1495-1555), Girolamo Muzio (1496-1576), Alessandro Citolini (ca. 1500-1582), Sperone Speroni (1500-1588), Benedetto Varchi (1503-1565), Ludovico Castelvetro (1505-1571). 
Coseriu, E. (1973): Probleme der strukturellen Semantik. Vorlesung gehalten im Wintersemester 1965/66 an der Universität Tübingen. Autorisierte und bearbeitete Nachschrift von Dieter Kastovsky. Tübingen: Narr (= Tübinger Beiträge zur Linguistik, 40).

Coseriu, E. \& Meisterfeld, R. (2003): Geschichte der romanischen Sprachwissenschaft. Band 1: Von den Anfängen bis 1492. Tübingen: Narr.

Ellena, S. (2011): Die Rolle der norditalienischen Varietäten in der ,,questione della lingua “. Eine diachrone Untersuchung zu Sprachbewusstsein, Sprachwissen und Sprachbewertung. Berlin \& Boston: de Gruyter (= Beihefte zur Zeitschrift für Romanische Philologie, 357).

Faithfull, G.R. (1953): The Concept of 'Living Language' in Cinquecento Vernacular Philology. The Modern Language Review 48. 278-292.

Ferguson, C.A. (1959): Diglossia. Word 15. 325-340.

Fishman, J.A. (1965): Who Speaks What Language to Whom and When? La Linguistique 2. 67-88.

Fishman, J.A. (1967): Bilingualism with and without Diglossia, Diglossia with and without Bilingualism. The Journal of Social Issues 23/2: Problems of Bilingualism. 29-38.

Fleischman, S. (1990): Philology, Linguistics, and the Discourse of the Medieval Text. Speculum 65. 19-37.

Fubini, R. (1961): La coscienza del latino negli umanisti. ,An latina lingua Romanorum esset peculiar idioma'. Studi medievali II/2 (dicembre). 505-550.

Holtus, G. (1989): Das ,vulgare illustre‘ als Modell einer italienischen Kunstsprache: Standard, Substandard und Varietät in Dante Alighieris Traktat ,De vulgari eloquentia' (1305). In: Holtus, G. \& Radtke, E. (Hg.), Sprachlicher Substandard II. Standard und Substandard in der Sprachgeschichte und in der Grammatik. Tübingen: Niemeyer. 1-13.

Ineichen, G. (1973): Das Verhältnis Dantes zur Sprache. Deutsches Dante-Jahrbuch 48. 63-78.

Klein, H.-W. (1957): Latein und Volgare in Italien. Ein Beitrag zur Geschichte der italienischen Nationalsprache. München: Hueber (= Münchner Romanistische Arbeiten, 12).

Kloss, H. ( $\left.{ }^{2} 1978\right):$ Die Entwicklung neuer germanischer Kultursprachen seit 1800. Düsseldorf: Pädagogischer Verlag Schwann (= Sprache der Gegenwart. Schriften des Instituts für deutsche Sprache, 37) [1. Aufl.: München: Pohl \& Co. 1952 (= Schriften des Goethe-Instituts, 1)].

Koch, P. \& Oesterreicher, W. (22011): Gesprochene Sprache in der Romania: Französisch, Italienisch, Spanisch. Tübingen: Niemeyer (= Romanistische Arbeitshefte, 31) [1. Aufl. 1990].

Koch, P. (1988): Italienisch: Externe Sprachgeschichte. In: Holtus, G., Metzeltin, M. \& Schmitt, C. (Hg.), Lexikon der Romanistischen Linguistik. Band IV: Italienisch, Korsisch, Sardisch. Tübingen: Niemeyer. 343-360.

Lüdtke, H. (2005): Der Ursprung der romanischen Sprachen. Eine Geschichte der sprachlichen Kommunikation. Kiel: Westensee (= Dialectologia Pluridimensionalis Romanica, 14).

Marazzini, C. (1993): La speculazione linguistica nella tradizione italiana. Le teorie. In: Serianni, L. \& Trifone, P. (Hg.), Storia della lingua italiana. Band I: I luoghi della codificazione. Torino: Einaudi. 231-329.

Marazzini, C. (2013): Da Dante alle lingue del Web. Otto secoli di dibattiti sull'italiano. Nuova Edizione. Roma: Carocci (= Studi superiori) [Original: Da Dante alla lingua selvaggia. Sette secoli di dibattiti sull'italiano. Roma: Carocci 1999].

Marcellino, G. \& Ammannati, G. (2015): Il latino e il 'volgare' nell'antica Roma. Biondo Flavio, Leonardo Bruni e la disputa umanistica sulla lingua degli antichi Romani. Pisa: Edizioni della Normale (= Testi e commenti, 17).

Marchiò, M. B. (2008): Come discutevano gli umanisti. Una disputa quatrocentesca sulla lingua parlata dai romani antichi. Firenze: Firenze Atheneum (= Collezione Oxenford. Universale Atheneum).

Mazzocco, A. (1993): Linguistic Theories in Dante and the Humanists: Studies of Language and Intellectual History in late Medieval and Early Renaissance Italy. Leiden: Brill.

Michel, A. (2005): Italienische Sprachgeschichte. Hamburg: Kovač (= Philologia - Sprachwissenschaftliche Forschungsergebnisse, 78).

Migliorini, B. ( ${ }^{12}$ 2007): Storia della lingua italiana. Introduzione di Ghino Ghinassi. Milano: Bompiani (= Saggi tascabili, 31).

Oesterreicher, W. (1998): Textzentrierung und Rekontextualisierung: Zwei Grundprobleme der diachronischen Sprach- und Textforschung. In: Ehler, C. \& Schaefer, U. (Hg.), Verschriftung und Verschriftlichung. Aspekte des Medienwechsels in verschiedenen Kulturen und Epochen. Tübingen: Narr (= ScriptOralia, 94). 10-39.

Passarelli, M.A. (1999): La lingua della patria. Leon Battista Alberti e la questione del volgare. Rom: Bagatto.

Patota, G. (1993): I percorsi grammaticali. In: Serianni, L. \& Trifone, P. (Hg.), Storia della lingua italiana. Band I: I luoghi della codificazione. Torino: Einaudi. 93-137.

Patota, G. (1999): Lingua e linguistica in Leon Battista Alberti. Rom: Bulzoni. 
Pistolesi, E. (2000): L'origine del concetto di ,latino volgare' negli studi romanzi: il caso di Celso Cittadini. In: Englebert, A., Pierrad, M., Rosier, L. \& van Raemdonck, D. (Hg.), Actes du XXIIe congrès international de linguistique et de philologie romanes. Bruxelles, 23-29 juillet 1998. Vol. 1. Tübingen: Niemeyer (= L'histoire de la linguistique, médiatrice de theories. Travaux de la section „Histoire de la linguistique”). $113-120$

Raffarin, A. (Hg.) (2015): Flavio Biondo, Leonardo Bruni, Poggio Bracciolini, Lorenzo Valla. Débats humanistes sur la langue parlée dans l'antiquité. Paris: Les Belles Lettres.

Reutner, U. \& Schwarze, S. (2011): Geschichte der italienischen Sprache. Eine Einführung. Tübingen: Narr (= Narr Studienbücher).

Schlemmer, G. (1983): Einleitung. In: Celso Cittadini: Trattato della vera origine, e del processo, e nome della nostra Lingua, scritto in vulgar Senese. Venetia 1601. Nachdruck mit einer Einleitung und Bibliographie von Gerd Schlemmer. Hamburg: Buske (= Romanistik in Geschichte und Gegenwart 13). 9-33.

Schöntag, R. (2016): L'architettura del latino nel Trattato della vera origine di Celso Cittadini. In: Becker, M. \& Fesenmeier, L. (Hg.), Relazioni linguistiche. Strutture, rapporti, genealogie. Frankfurt a.M.: Lang (= Studia romanica et linguistica, 45). 11-30.

Schunck, M. (2003): Der Sprachwandel im metalinguistischen Diskurs Italiens und Frankreichs von der Renaissance zur Aufklärung. Frankfurt a.M. (= Studien zur Allgemeinen und Romanischen Sprachwissenschaft, 9).

Sinner, C. (2014): Varietätenlinguistik. Eine Einführung. Tübingen: Narr (= Narr Studienbücher).

Strauss, F. (1938): Vulgärlatein und Vulgärsprache in Zusammenhang der Sprachenfrage im 16. Jahrhundert (Frankreich und Italien). Marburg a.d. Lahn: Michaelis-Braun (= Marburger Beiträge zur Romanischen Philologie, 21).

Tavoni, M. (1982): The 15th-Century Controversy on the Language Spoken by the Ancient Romans: An Inquiry into Italian Humanist Concepts of ,Latin“, ,Grammar', and ,Vernacular'. Historiographia linguistica 9. 237 264.

Tavoni, M. (1984): Latino, grammatica, volgare. Storia di una questione umanistica. Padova: Antenore (= Medioevo e umanesimo, 53)

Valle, V. della \& Patota, G. (2006): L'italiano. Milano: Sperling \& Kupfer (= Le Grandi Guide).

Veith, W.H. (2002): Soziolinguistik. Ein Arbeitsbuch mit 100 Abbildungen sowie Kontrollfragen und Antworten. Tübingen: Narr (= Narr Studienbücher).

Vitale, M. ( $\left.{ }^{4} 1967\right)$ : La questione della lingua. Palermo: Palumbo (= Storia della critica, 3) [1. Aufl. 1960].

Ward, M.T. (1990): Celso Cittadini and the Origin of the Vernacular: The Convergence of Science and Subjectivity. In: Joseph, J.E. \& Taylor, T J. (Hg.), Ideologies of Language. London \& New York: Routledge (= Routledge Politics of Language Series). 97-111.

Werner, E. (1999): Ansätze einer historisch-vergleichenden Sprachbetrachtung im 17. Jahrhundert in Italien: Celso Cittadini. In: Haßler, G. \& Schmitter, P. (Hg.): Sprachdiskussion und Beschreibung von Sprachen im 17. und 18. Jahrhundert. Münster: Nodus (= Studium Sprachwissenschaft Beiheft, 32). 351-369.

Wunderli, P. (1994): Dante - ein Linguist. Dante-Jahrbuch 68/69. 81-126.

\section{Quellen}

Alberti, Della fam. $=$ Alberti, Leon Battista ( $\left.{ }^{2} 1994\right)$ : Proemio del libro terzo. In: Alberti, Leon Battista: I libri della famiglia. A cura di Ruggiero e Alberto Tenenti. Nuova edizione a cura di Francesco Furlan. Torino: Einaudi (= Nuova Universale Einaudi) [1. Aufl. 1969]. 187-192.

Bembo, Prose $=$ Bembo, Pietro $\left({ }^{2} 1966\right)$ : Prose della volgar lingua. In: ibid.: Prose e rime. A cura di Carlo Dionisotti. Torino: UTET (=Classici italiani) [1. Aufl. 1960]. 71-309.

Biondo, De verb. rom. = Biondo, Flavio (2015): De verbis Romanae locutionis Blondi ad Leonardum Aretinum. In: Marcellino, Ammannati, Giulia (2015): Il latino e il 'volgare' nell'antica Roma. Biondo Flavio, Leonardo Bruni e la disputa umanistica sulla lingua degli antichi Romani. Pisa: Edizioni della Normale (= Testi e commenti, 17). 148-183.

Bracciolini, Utrum prisc. $=$ Bracciolini, Poggio (2008): Utrum priscis romanis latina lingua omnibus communis fuerit, an alia quaedam doctorum virorum, alia plebis et vulgi, tertiae convivalis historiae disceptatio. In: Marchiò, Mario Bianco: Come discutevano gli Umanisti. Una disputa quattrocentesca sulla lingua parlata dai romani antichi. Firenze: Atheneum. 185-204.

Bruni, An vulg. et lit. = Bruni, Leonardo (2015): Leonardus Flavio Foroliviensi S. quaerit an vulgus et literati eodem modo per Terentii Tulliique tempora Romae locuti sint. In: Marcellino, Ammannati, Giulia (2015): Il 
latino e il 'volgare' nell'antica Roma. Biondo Flavio, Leonardo Bruni e la disputa umanistica sulla lingua degli antichi Romani. Pisa: Edizioni della Normale (= Testi e commenti, 17). 238-249.

Cittadini, Tratt. = Cittadini, Celso (1983): Trattato della vera origine, e del processo, e nome della nostra Lingua, scritto in vulgar Senese. Venetia 1601. Nachdruck mit einer Einleitung und Bibliographie von Gerd Schlemmer. Hamburg: Buske (= Romanistik in Geschichte und Gegenwart (RomGG), 13) [Prima edizione: Venezia 1601].

Dante, DVE = Alighieri, Dante (2007): De vulgari eloquentia. Mit der italienischen Übersetzung von Gian Giorgio Trissino (1529). Deutsche Übersetzung von Michael Frings und Johannes Kramer. Stuttgart: ibidem.

Guarino, De ling. lat. diff. = Veronese, Guarino (2008): Guarinus Veronensis ill. principi Leonello Marchioni Estensi de lingue latine differentiis. In: Marchiò, Mario Bianco: Come discutevano gli Umanisti. Una disputa quattrocentesca sulla lingua parlata dai romani antichi. Firenze: Atheneum. 174-184.

Valla, Apologus = Valla, Lorenzo (2008): Dall ,Apologus'. Secundus Actus. In: Marchiò, Mario Bianco: Come discutevano gli Umanisti. Una disputa quattrocentesca sulla lingua parlata dai romani antichi. Firenze: Atheneum. 205-239. 
